Andrews University

Digital Commons @ Andrews University

\title{
Coping Skills, Emotional Intelligence, Attachment, and Resilience on Post-Traumatic Stress Disorder in Private Sector Emergency Medical Technicians
}

Brenda-Joyce Gonzales Orozco Markert-Green

Andrews University, markertb@andrews.edu

Follow this and additional works at: https://digitalcommons.andrews.edu/dissertations

Part of the Emergency Medicine Commons, and the Psychiatric and Mental Health Commons

\section{Recommended Citation}

Markert-Green, Brenda-Joyce Gonzales Orozco, "Coping Skills, Emotional Intelligence, Attachment, and Resilience on Post-Traumatic Stress Disorder in Private Sector Emergency Medical Technicians" (2021). Dissertations. 1748.

https://digitalcommons.andrews.edu/dissertations/1748

https://dx.doi.org/10.32597/dissertations/1748

This Dissertation is brought to you for free and open access by the Graduate Research at Digital Commons @ Andrews University. It has been accepted for inclusion in Dissertations by an authorized administrator of Digital Commons@ Andrews University. For more information, please contact repository@andrews.edu. 


\section{ABSTRACT}

COPING SKILLS, EMOTIONAL INTELLIGENCE, ATTACHMENT, AND RESILIENCE ON POST-TRAUMATIC STRESS DISORDER IN PRIVATE SECTOR EMERGENCY MEDICAL TECHNICIANS

by

Brenda-Joyce G. Orozco Markert-Green, M.A., DMFT

Chair: Dennis Waite 


\section{ABSTRACT OF GRADUATE STUDENT RESEARCH}

Dissertation

Andrews University

School of Education

Title: COPING SKILLS, EMOTIONAL INTELLIGENCE, ATTACHMENT, AND RESILIENCE ON POST-TRAUMATIC STRESS DISORDER IN PRIVATE SECTOR EMERGENCY MEDICAL TECHNICIANS

Name of researcher: Brenda-Joyce G. Orozco Markert-Green, M.A., DMFT

Name and degree of faculty chair: Dennis Waite, Ed.D.

Date completed: May 2021

Problem

Private sector Emergency Medical Technicians (EMTs) are first responders who respond to traumatic incident scenes along with firefighters and police officers.

However, news agencies and the public do not acknowledge private sector EMTs with the same vigor or accolade as public sector EMTs, nor are the risks to life, health, and mental well-being recognized among this population. Thus, the research literature has been sparse regarding how private sector EMTs experience PTSD, and what unique risk factors and needs may be present.

\section{Purpose Statement}

The purpose of this study was to examine the relationship between PTSD symptoms and coping skills, emotional intelligence, attachment, and resilience among private sector EMT workers. 
Method

Utilizing a mixed-methods design, 50 private sector EMTs recruited through social media, word of mouth, and Michigan State DHHS completed the: PTSD Checklist (PCL-5), Brief COPE, Schutte Emotional Intelligence Scale (SEIS), Measure of Attachment Qualities (MAQ), and Connor Davidson-Resilience Scale (CD-RISC) to explore the relationship between PTSD and coping skills, emotional intelligence, attachment, and resiliency among private sector EMTs. Three private sector EMTs participated in qualitative interviews to identify their experiences, risk factors, and needs as private sector EMTs.

\section{Results}

Thirty percent of the EMTs (21\% of EMT-Basics; $40 \%$ of EMT-Paramedics) met PCL-5 criteria for PTSD. Based on DSM-5 criteria, 36\% of the EMTs (40\% of EMTBasics; 30\% of EMT-Paramedics) meeting criteria for PTSD. Results showed no difference in the severity in which EMT- Basics and EMT- Paramedics experience PTSD. Both EMT- Basics and EMT- Paramedics use Positive Thinking as their primary method of coping. Fifty-eight percent of EMTs fell into the average range on emotional intelligence, and 30\% fell into the unusually high range. The EMTs demonstrated the greatest struggle in being able to utilize their own emotions, finding it much easier to perceive emotions and to manage their own and others' emotions. The EMT- Paramedics fell into the highest $25 \%$ and EMT- Basics fell into the lowest $25 \%$ of the population on resiliency, EMT- Basics were nearly one-and-three-fourths times less resilient than EMTParamedics. Most of the EMTs were securely attached; however, EMT- Basics demonstrated significantly more ambivalent attachment and significantly more anxious 
attachment. The most significant factors predicting PTSD were the use of avoidance coping and having ambivalent and anxious attachment styles. Regression analysis suggested that higher PTSD scores would be seen in EMTs with higher scores in Avoidance Coping and Ambivalence Merger (Anxious) Ambivalence but lower scores in Ambivalence-Worry (Ambivalent) Attachment scores, with the most important predictor being Avoidance Coping. Individual interviews revealed five overarching themes related to the experiences of private sector EMTs: work environment, work quality/altruism, disenfranchisement/societal acknowledgement, resiliency, and contributors to PTSD.

Conclusions

The experience of private sector EMTs is clearly one of disenfranchisement, which is likely to impact their rates of PTSD. Additional research needs to be undertaken and public education initiated in relation to this population, as well as therapeutic focus on the disenfranchisement component in conjunction with the PTSD treatment. 
Andrews University

School of Education

COPING SKILLS, EMOTIONAL INTELLIGENCE, ATTACHMENT, AND RESILIENCE ON POST-TRAUMATIC STRESS DISORDER IN PRIVATE SECTOR EMERGENCY MEDICAL TECHNICIANS

\author{
A Dissertation \\ Presented in Partial Fulfillment \\ of the Requirements for the Degree \\ Doctor of Philosophy
}

by

Brenda-Joyce G. Markert-Green M.A., DMFT

May 2021 
(C) Copyright by Brenda-Joyce G. Markert-Green, M.A., DMFT 2021 All Rights Reserved 
COPING SKILLS, EMOTIONAL INTELLIGENCE, ATTACHMENT, AND RESILIENCE ON POST-TRAUMATIC STRESS DISORDER IN PRIVATE SECTOR EMERGENCY MEDICAL TECHNICIANS

\author{
A dissertation \\ presented in partial fulfillment \\ of the requirements for the degree \\ Doctor of Philosophy
}

by

Brenda-Joyce G. Markert-Green, M.A., DMFT

APPROVAL BY THE COMMITTTEE:

Chair: Dennis Waite

Member: Jimmy Kijai

Member: Harvey Burnett

External: Melissa Ponce-Rodas
Dean, School of Education

Alayne Thorpe 
I dedicate this work and my life to my Creator, Sustainer, and Lord. May my life be a true and accurate representation of who You are! 


\section{TABLE OF CONTENTS}

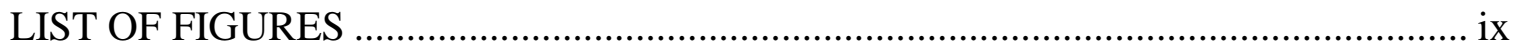

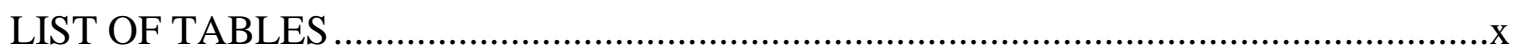

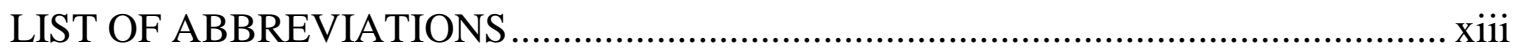

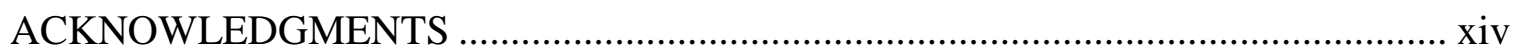

\section{Chapter}

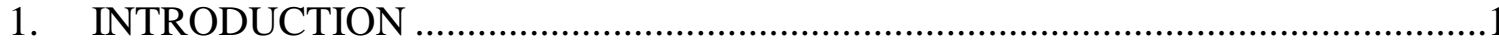

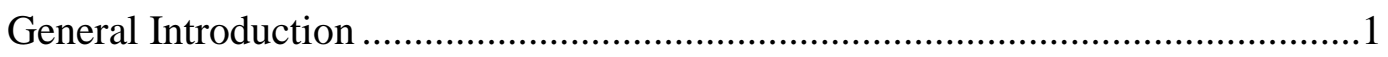

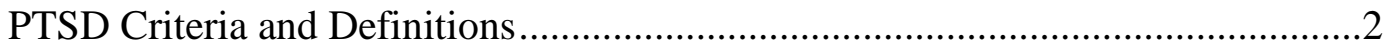

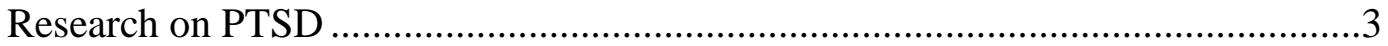

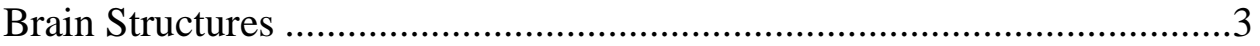

Physical and Mental Health ....................................................................

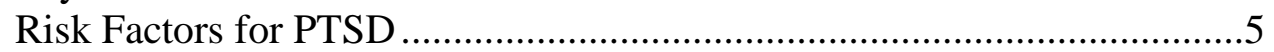

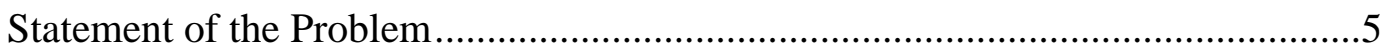

Coping Skills ...................................................................................

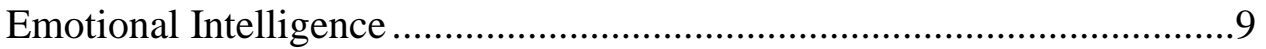

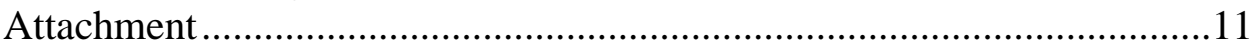

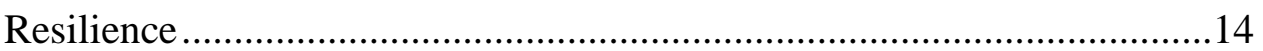

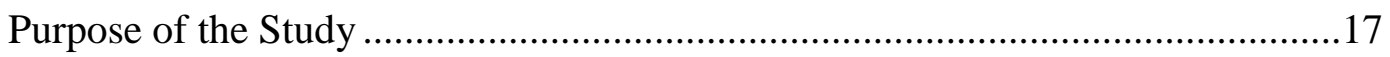

Four Factor Model ……..............................................................................

Theoretical Background and Assumptions ...............................................18

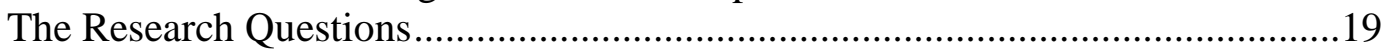

Quantitative Research Questions ...........................................................19

Qualitative Interview Questions ………………………......................20

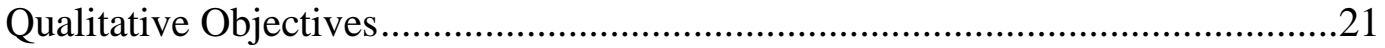

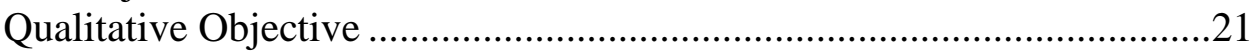

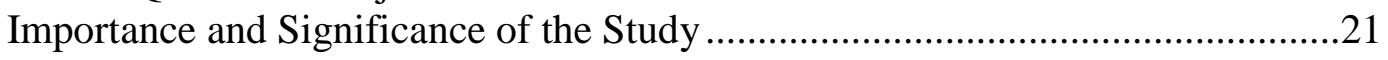

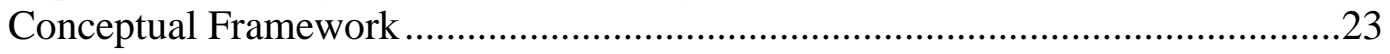

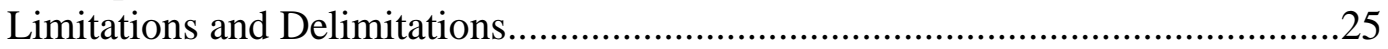

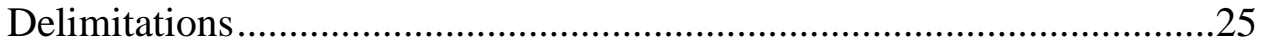

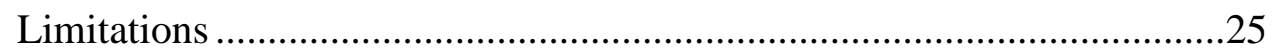

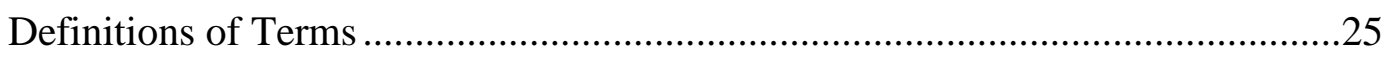

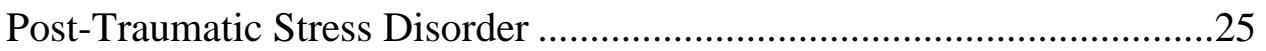

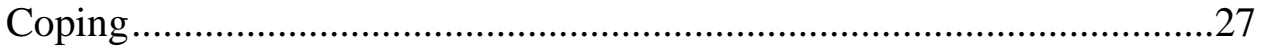

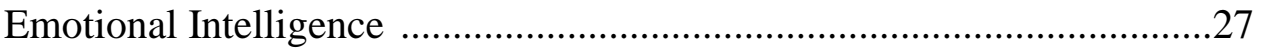

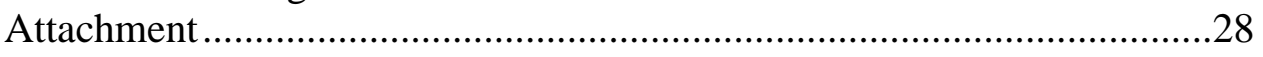

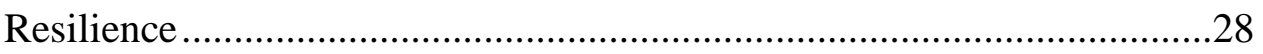

Emergency Medical Technician .............................................................28

Emergency Medical Technician - Basic's.....................................28

Emergency Medical Technician - Intermediate's ...........................28 


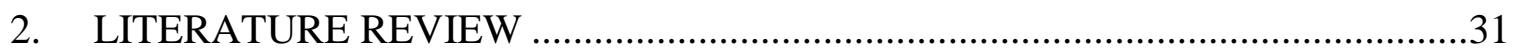

Post-Traumatic Stress Disorder in Field Emergency Medical Technicians ...........31

Data Sources and Searches .....................................................................31

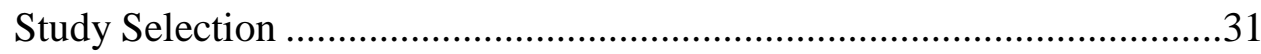

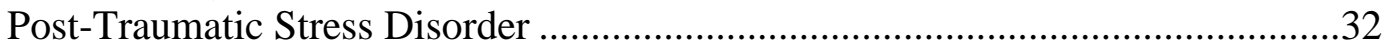

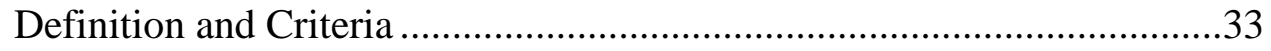

Impact of Post-Traumatic Stress Disorder ............................................................34

Biological Factors in Post-Traumatic Stress Disorder..............................................35

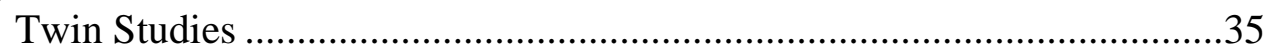

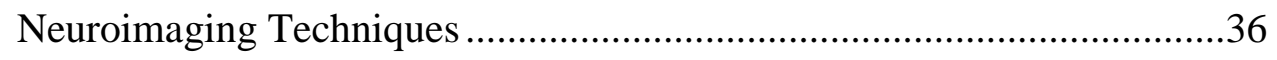

Affected Brain Structures ...................................................................38

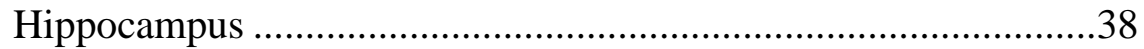

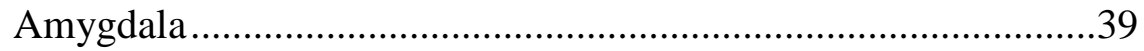

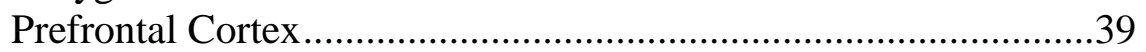

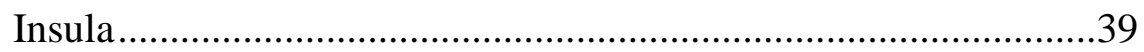

Hypothalamic-Pituitary-Adrenal Axis ........................................... 40

Cingulate Cortex .....................................................................

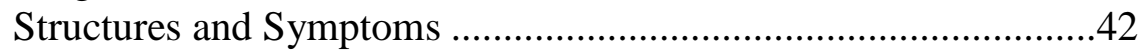

Childhood Factors ................................................................................

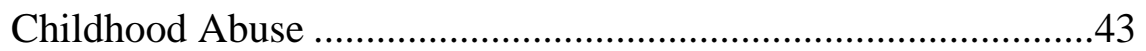

Risk Factors for PTSD ......................................................................

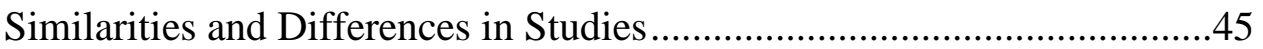

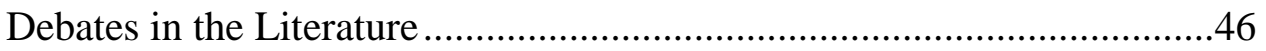

Psychological Factors in Post-Traumatic Stress Disorder .....................................48

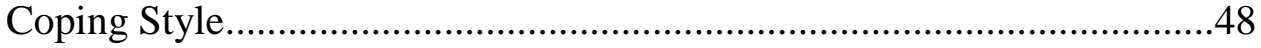

History and Foundation...............................................................

Previous Decade...................................................................

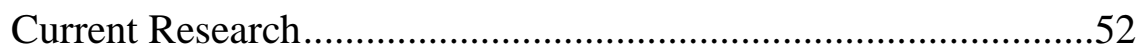

Emotional Intelligence …………………………………....................53

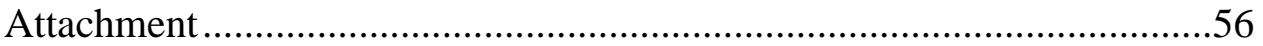

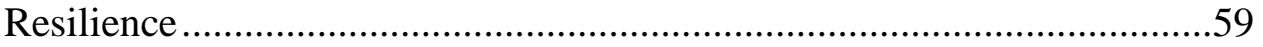

Vulnerability to Developing PTSD ..........................................................61

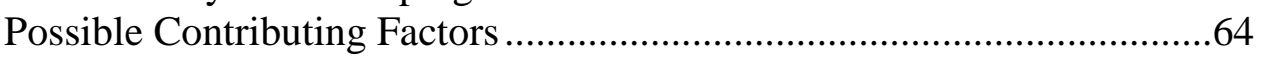

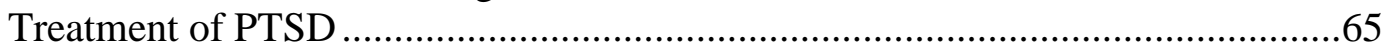

Epidemiology and Prevalence Rates of PTSD ................................................65

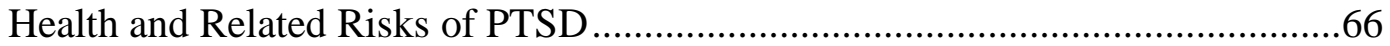

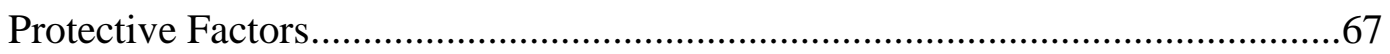

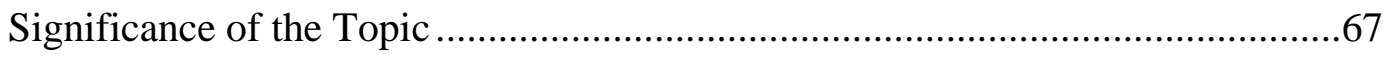

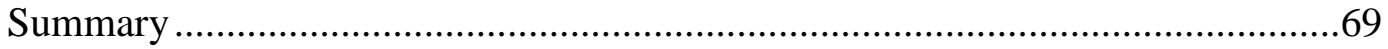




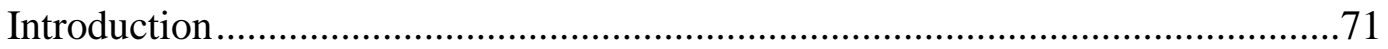

Purpose of the Study ...................................................................... 71

Organization of the Chapter............................................................. 71

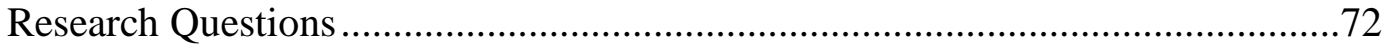

Qualitative Interview Questions ......................................................72

Quantitative Research Questions .......................................................72

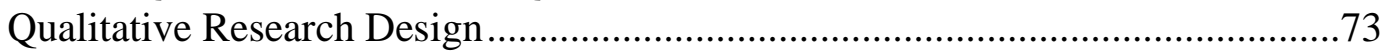

Qualitative Objective .......................................................................... 74

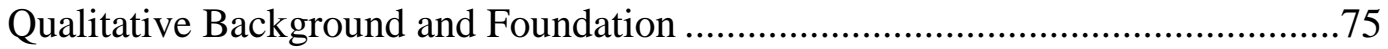

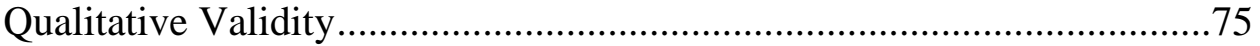

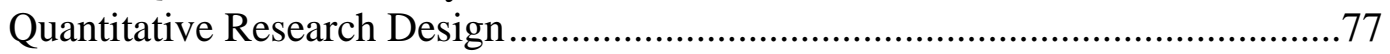

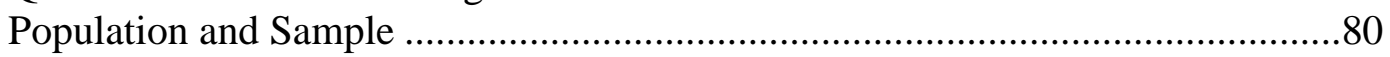

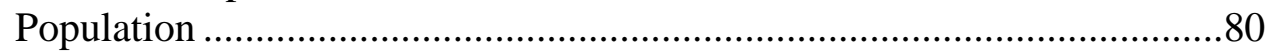

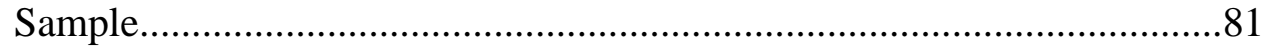

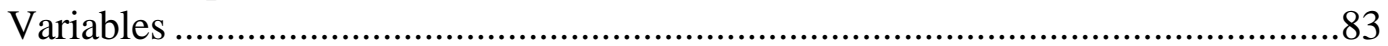

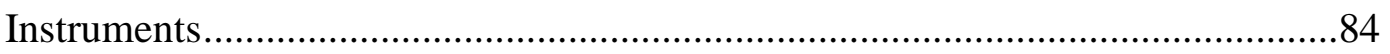

Post-Traumatic Stress Disorder ......................................................8

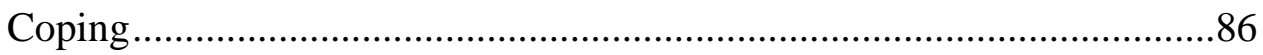

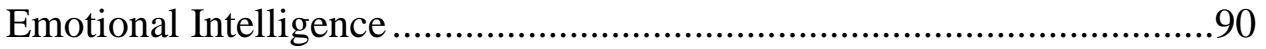

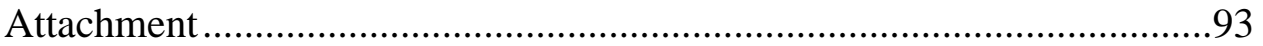

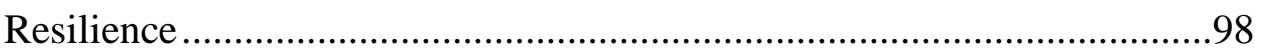

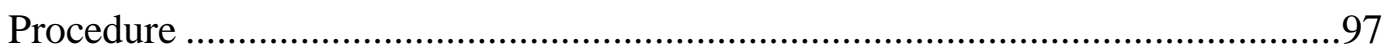

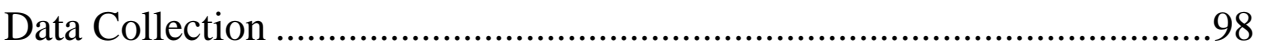

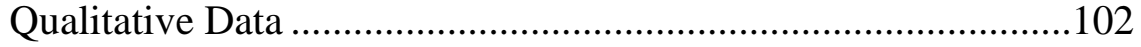

Data Cleaning and Preparation ............................................................103

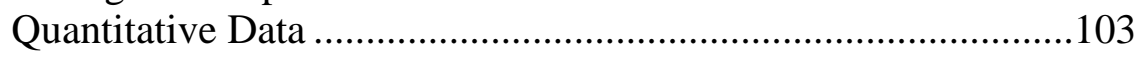

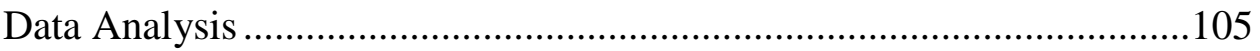

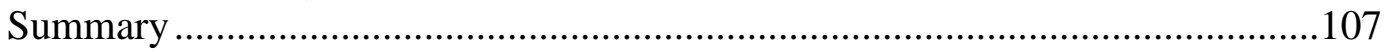

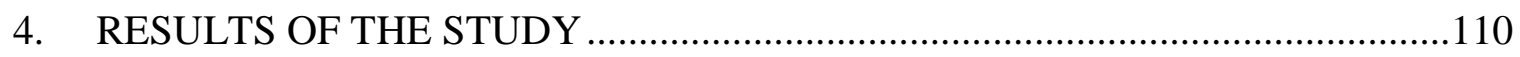

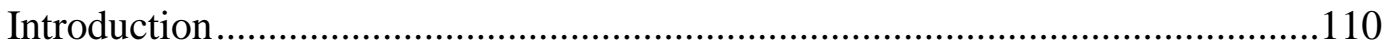

Sample for the Qualitative Portion of the Study ..............................................110

Description of the Variables ..........................................................................112

Individual Interview Questions .....................................................112

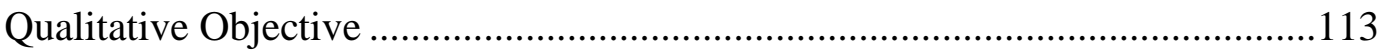

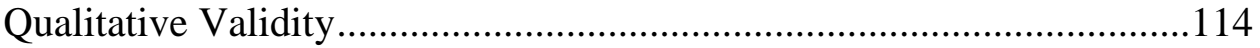

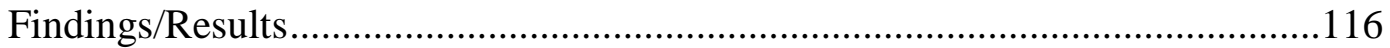

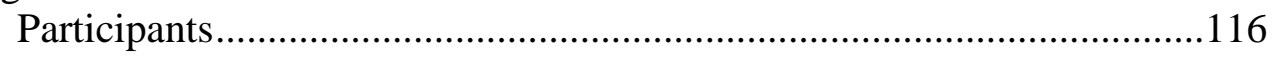

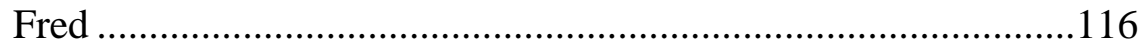

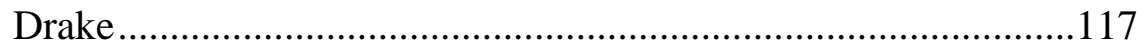

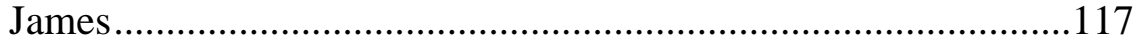


Qualitative Interview Questions and Themes ...................................................117

Qualitative Interview Question One ....................................................117

Qualitative Interview Question Two.....................................................117

Qualitative Interview Question Three......................................................118

Qualitative Interview Question Four .....................................................118

Qualitative Interview Question Five ....................................................119

Qualitative Interview Question Six.......................................................120

Qualitative Interview Question Seven .................................................120

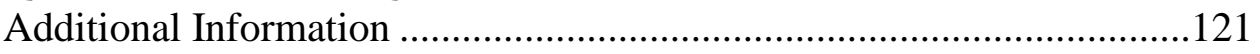

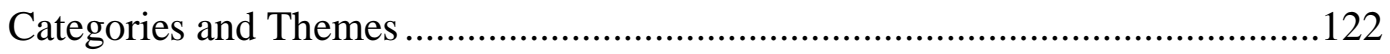

Theme 1: Work Environment ………………………………….......122

Theme 2: Work Quality/Altruism.........................................................123

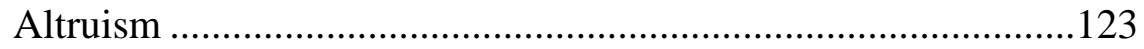

Theme 3: Disenfranchisement ..........................................................124

Societal Acknowledgement ......................................................124

Theme 4: Resiliency ……………………………………................125

Theme 5: Contributions to PTSD …………………………………....125

Sample for the Quantitative Portion of the Study …….....................................126

Participant Demographics ....................................................................127

Description of the Variables and Instrument Reliability ....................................128

Post-Traumatic Stress Disorder .............................................................128

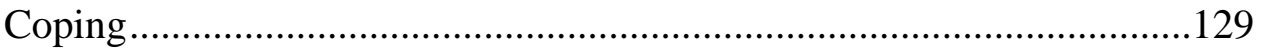

Emotional Intelligence …………………………………………....130

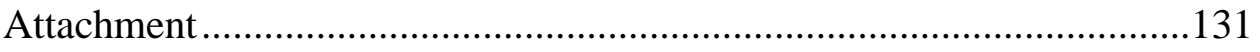

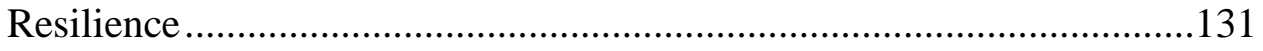

Research Questions and Evaluation.............................................................132

Research Question One ...................................................................132

Research Question Two ....................................................................135

Research Question Three ......................................................................138

Research Question Four .....................................................................141

Emotional Intelligence ............................................................141

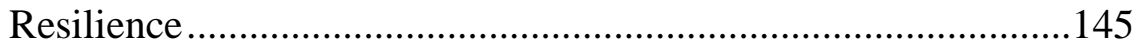

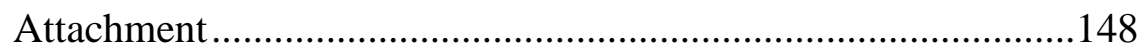

Research Question Five .................................................................151

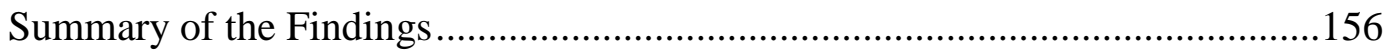

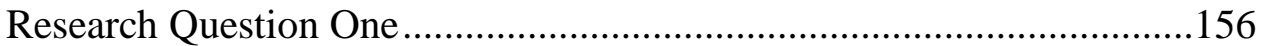

Research Question Two .....................................................................156

Research Question Three ....................................................................156

Research Question Four ....................................................................156

Emotional Intelligence ..........................................................156

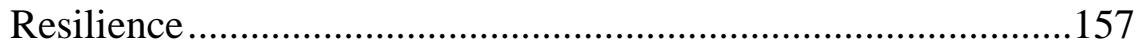

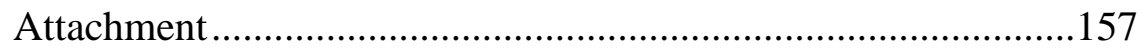

Research Question Five .................................................................158 
Integration of Quantitative and Qualitative Results............................................159

Areas of Agreement ........................................................................159

Areas of Disagreement.........................................................................160

New Themes Identified in the Qualitative Data ......................................160

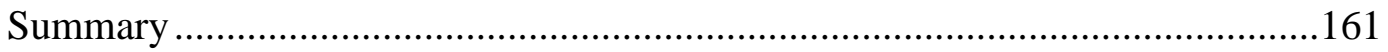

5. SUMMARY, DISCUSSION, AND RECOMMENDATIONS ………………........164

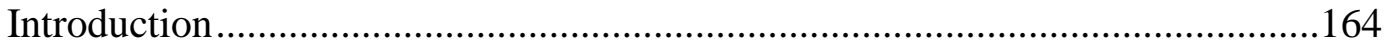

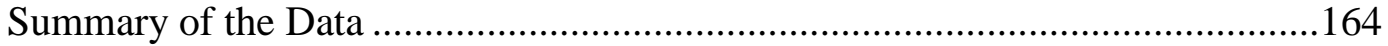

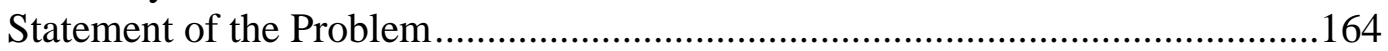

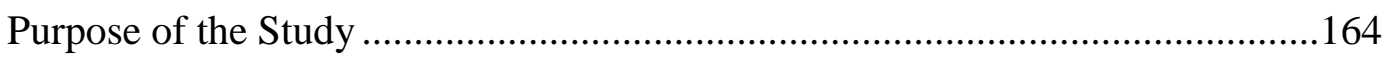

Literature Review Summary ………………………...................................165

Impact of Post-Traumatic Stress Disorder .............................................165

Psychological Factors in Post-Traumatic Stress Disorder .....................................165

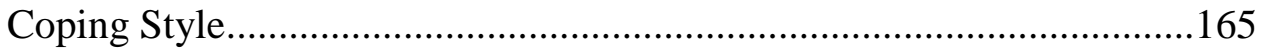

Emotional Intelligence .......................................................................166

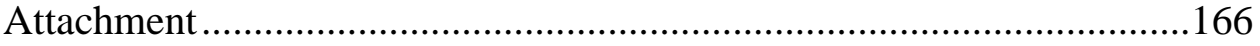

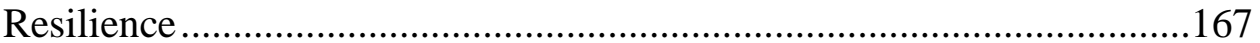

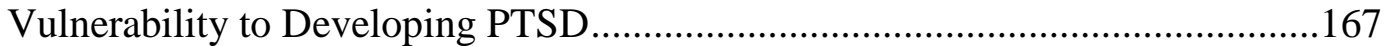

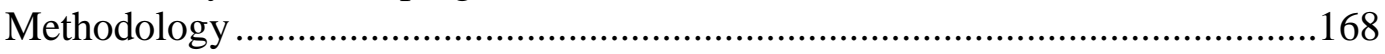

Qualitative Research Design ..............................................................168

Quantitative Research Design ..............................................................168

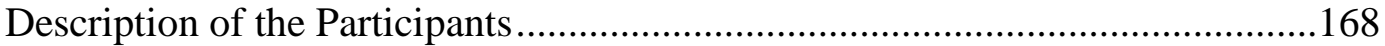

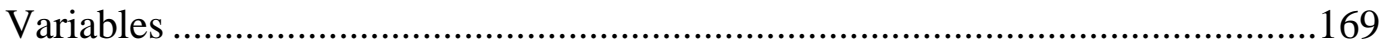

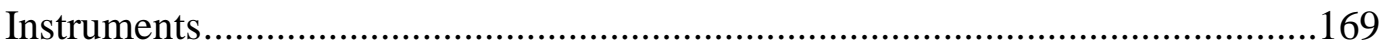

Post-Traumatic Stress Disorder ........................................................169

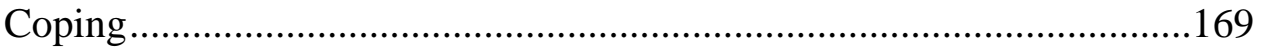

Emotional Intelligence .....................................................................169

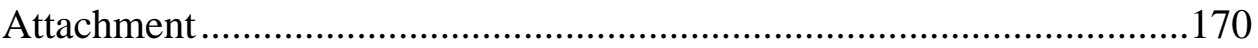

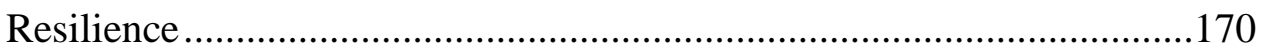

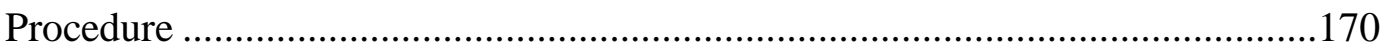

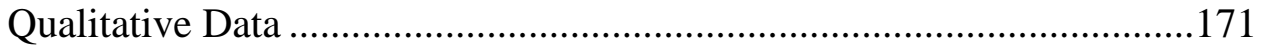

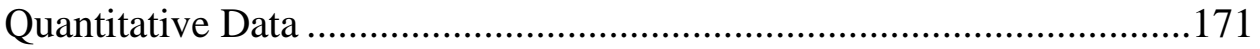

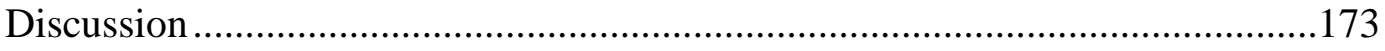

Quantitative Results ........................................................................173

Research Question One ..........................................................173

Research Question Two ..........................................................175

Research Question Three ..........................................................176

Research Question Four ..........................................................178

Emotional Intelligence ....................................................178

Resilience .................................................................179

Attachment .................................................................180

Research Question Five ..........................................................182 


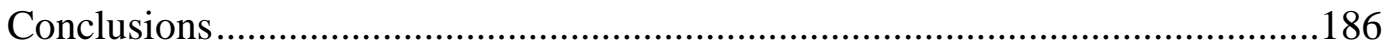

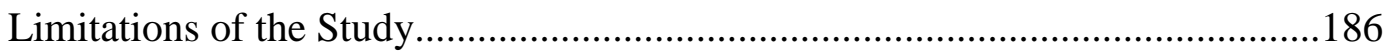

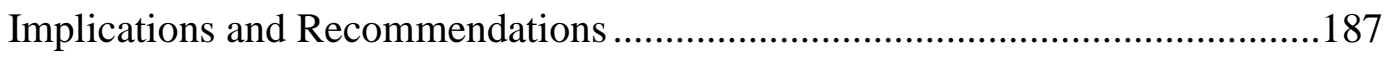

Implications for the Field .....................................................................187

Implications and Recommendations for Future Research .......................193

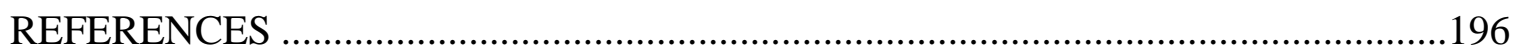

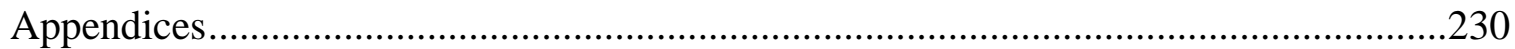

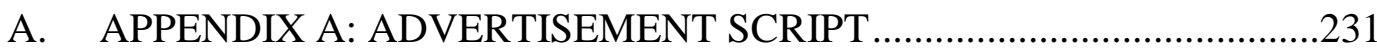

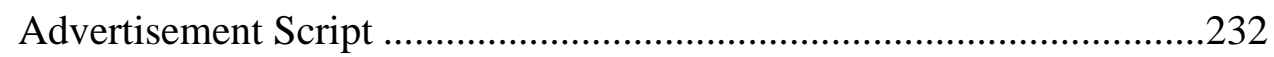

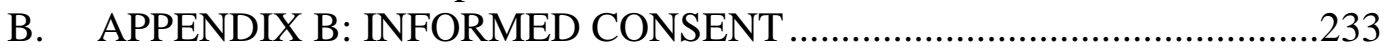

Informed Consent............................................................................234

C. APPENDIX C: DEMOGRAPHIC QUESTIONS ....................................237

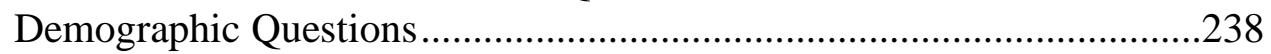

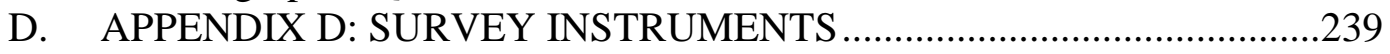

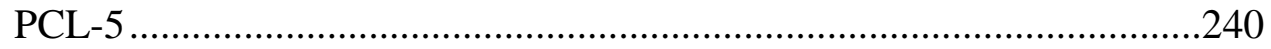

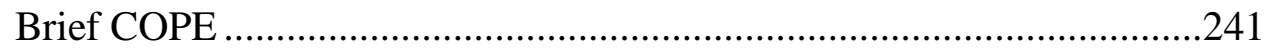

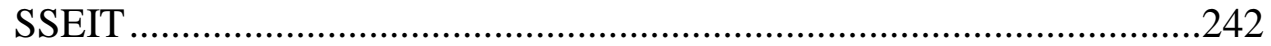

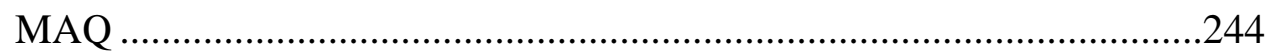

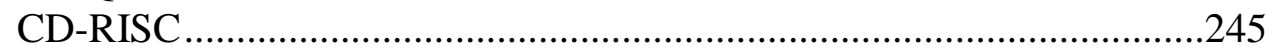




\section{LIST OF FIGURES}

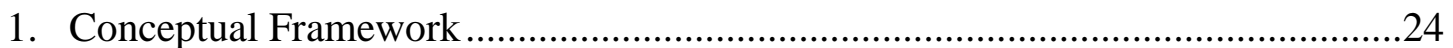




\section{LIST OF TABLES}

1. Brain Regions and Neurochemical Dysfunction Associated with PTSD ..............42

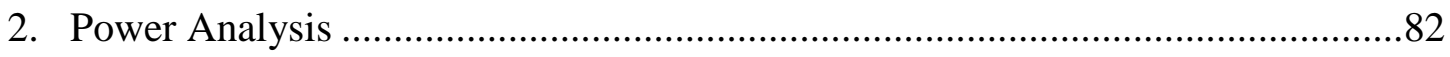

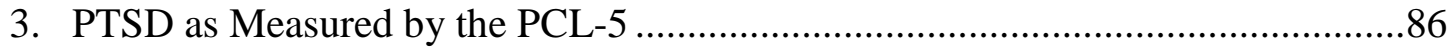

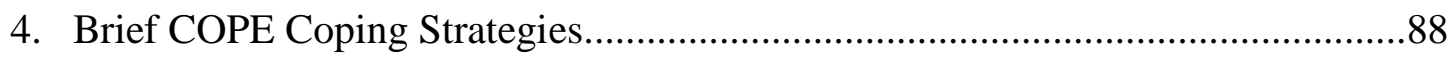

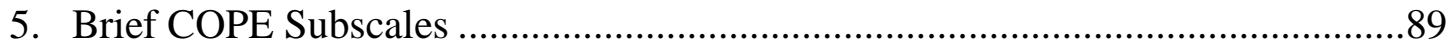

6. Validity and Reliability of the Brief COPE .................................................. 90

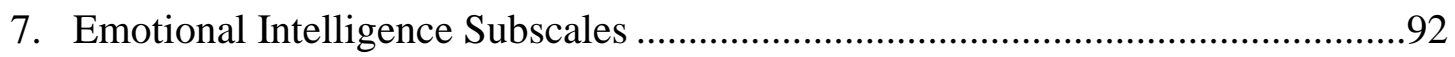

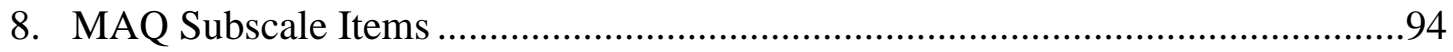

9. Measure of Attachment Qualities (MAQ) Validity ................................................ 95

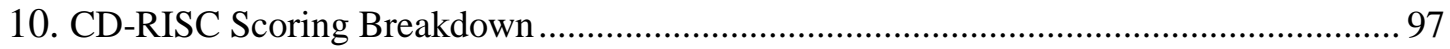

11. Qualitative Study Participant Demographics..................................................112

12. Sample for the Quantitative Portion of the Study ...........................................128

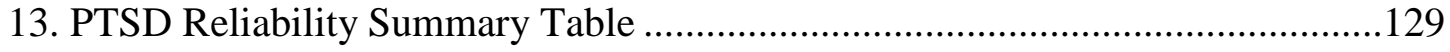

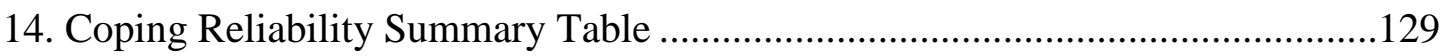

15. Emotional Intelligence Reliability Summary Table .......................................130

16. Attachment Reliability Summary Table ...................................................131

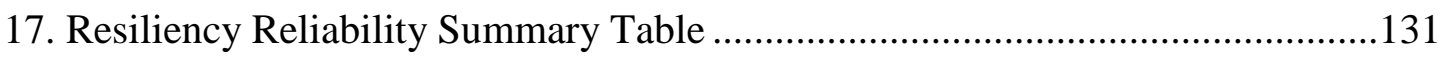

18. Overall Percentage of PTSD Endorsed Based on PCL-5 Criteria ......................133

19. Overall Percentage of PTSD Endorsed Based on DSM-5 Criteria.....................133

20. Percent of EMTs Endorsing DSM-5 Items ................................................. 134

21. Percent of EMTs Endorsing Criterion B Items ...............................................134

22. Percent of EMTs Endorsing Criterion C Items ..............................................134

23. Percent of EMTs Endorsing Criterion D Items.............................................135 
24. Percent of EMTs Endorsing Criterion E Items

25. EMT Group Differences on PCL-5 Scores........................................................136

26. EMT Group Differences on PCL-5 Cut-Off Scores ……………………….......136

27. EMT Group Differences on DSM-5 Criteria: 1 "B" Item; 1 "C" Item; 2 "D"

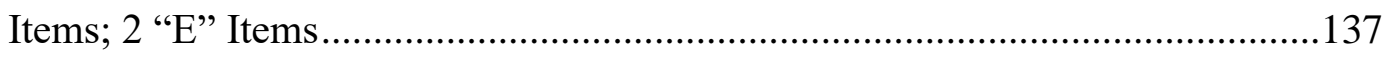

28. Comparison of EMT Groups Meeting Symptoms Based on DSM-5 Criterion...137

29. Descriptive Statistics of Coping Strategies........................................................138

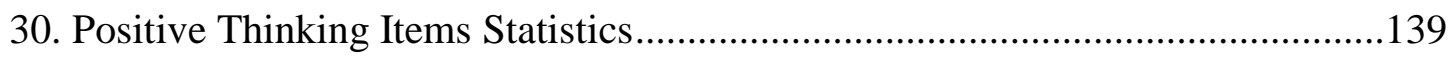

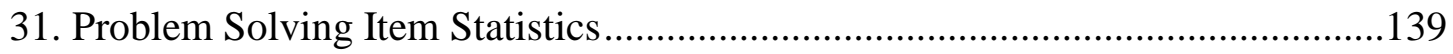

32. Use of Emotional Social Support Item Statistics ...............................................139

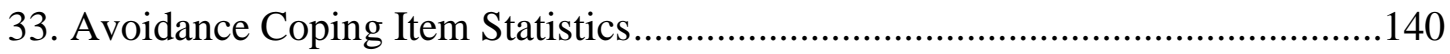

34. Group Means and Standard Deviation of Coping Strategies ...............................140

35. Schuette Emotional Intelligence Scale (SEIS) Scoring Breakdown .....................141

36. Descriptive Statistics of Emotional Intelligence Subscales ...................................142

37. Managing Own Emotions Item Statistics …………………………...............143

38. Managing Others' Emotions Item Statistics ........................................................143

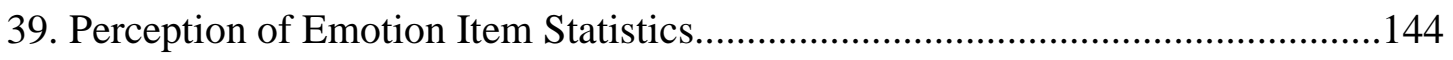

40. Utilization of Emotion Item Statistics...............................................................144

41. Group Means and Standard Deviations of Emotional Intelligence Subscales.....145

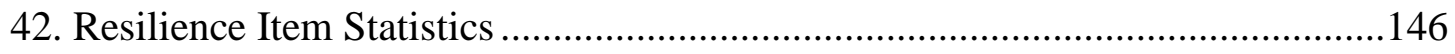

43. Connor Davidson Resiliency Scale (CD-RISC) Scoring Breakdown ..................146

44. Group Means and Standard Deviations of the CD-RISC Results.........................147

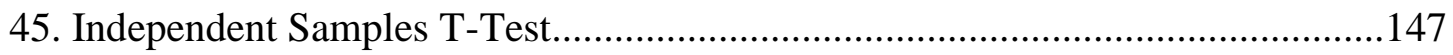




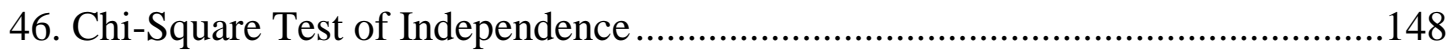

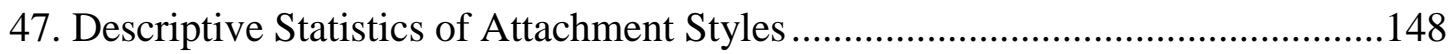

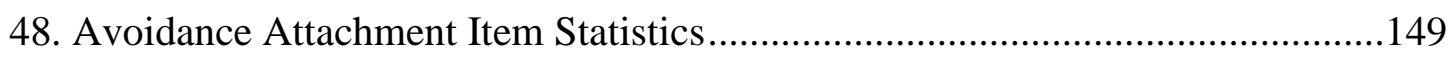

49. Ambivalence-Worry (Ambivalent) Attachment Item Statistics ........................149

50. Ambivalence-Merger (Anxious) Attachment Item Statistics ...........................149

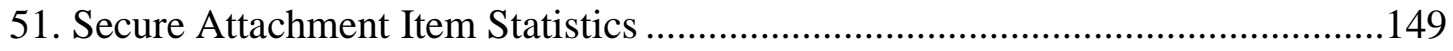

52. Group Means and Standard Deviations for Attachment Subscale Results ..........150

53. Multiple Linear Regression Variable Mean, Standard Deviation, and Bivariate

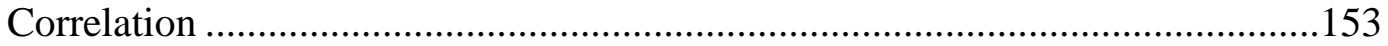

54. Regression Analysis Results - Full Model ................................................154

55. Regression Analysis Results - Restricted Model ...........................................155 


\section{LIST OF ABBREVIATIONS}

CD-RISC Connor Davidson-Resilience Scale

DSM-5 The Diagnostic and Statistical Manual of Mental Disorders $-5^{\text {th }}$ Edition

ED Emergency Department

EMT Emergency Medical Technician

EMT-B Emergency Medical Technician - Basic

EMT-I Emergency Medical Technician - Intermediate

EMT-P Emergency Medical Technician - Paramedic

MAQ Measure of Attachment Qualities

PCL-5 PTSD Checklist

PTSD Post-Traumatic Stress Disorder

SEIS Schutte Emotional Intelligence Scale

SREIT Self-Report Emotional Intelligence Test

SSEIT Schutte Self-Report Emotional Intelligence Test 


\section{ACKNOWLEDGMENTS}

There have been a number of people who have been instrumental not only in my life, but throughout this second doctoral process for me. The first of these is the definite leading of God in my life with regard to me pursuing this degree. If it wasn't for God not only impressing the desire upon my heart, but also opening impossible doors financially, I wouldn't be at this juncture in my life! I thank God for Her/His continual presence in my life, and for the hope of one day living in that divine presence for eternity!

I thank my mother, Joyce Markert, for her ever-present and continual prayers, and for being the prayer warrior that she is! It is because of her faith, and because of the Christian foundation that I was fortunate enough to have growing up, that I have come to be a woman of deep and abiding faith in our heavenly Creator! Thank you, Mom, for always being there for me! I love you!

To my husband, Mike, and the precious children I am blessed to have in my life, Annette-Suzanne, Brittany-Laurée, Courtney-Nathaniel, Brandi-Chantal, BrendanMichael, and Cody-Ryan, I thank you for your constant support as I pursued a better life for our family! I love you all with all of my heart! And to my sisters, Denise, Lorri, Michelle, Sheryl, Jacki, and Marsha, you are all in my continual prayers. I thank you for the ways you have been there for me throughout the years.

To my doctoral committee, I thank you for your guidance, advising, and feedback, and for staying with me to the end! To my Chairperson, Dr. Dennis Waite, I thank you for taking this on so close to retirement, for your belief in me, for your guidance, and for all your help with getting my final document together; to my statistician, Dr. Jimmy Kijai, thank you for your guidance with sometimes confusing material and for making it 
clear, and for pushing me to produce a strong document; and for my content expert, Dr. Harvey Burnett, thank you for always being available to talk to me, for brainstorming with me, and for the many hours you spent teasing this all out so that I could go forward with clarity and purpose! I cannot thank you enough! Dr. Grajales, thank you so much for stepping in and being so willing to help me with my statistics questions when you were not on my committee! Thank you for being so kind to me! I appreciate you going outside of your way to help this needy study, and for being so quick with your responses to me! To Dr. Alayne Thorpe, I cannot find the words to thank you for your help in moving me towards the finalization of my dissertation and the completion of my degree! You are such a kind, Christian woman! I hope you know how you impact the lives around you, and how you have made such an impact on me for the Kingdom of God! May the Lord bless you richly as you continue to serve the students of this institution in being the hands, feet, and heart of God in human form! To Dr. Woolford-Hunt, thank you for your consistent treatment of me as a seasoned professional, for your support throughout my program, and for your part in moving me toward completion of this second Doctorate for me! Finally, to Dr. Melissa Ponce-Rodas, I thank you for being my external examiner, for making me feel so comfortable during my Doctoral Defense, for your suggestions to strengthen my document, and for meeting with me to finalize my document!

To my many friends who have never given up on my educational endeavors, and for your continuous encouragement! I share the wealth of many friends who have played a deep and instrumental role in my life, and I thank each of you! To Rae Nelson-Beisel, Cindy Friend, Lisa Gillespie, Tami Cagle, Kim Berent, Lori Colwell, Jennifer Jones- 
Eidson, Lisa Harris, Karen Lafferty, Mindy MacKenzie, Norma Mixon, Heather McDaniel, Dana Noonan, Minerva Sajjadi, Michal Madison, Tricia Sigler, Salenna Rusello, Noell Giudice-Woodruff, and Zeljka Guzman, your friendships have enriched my life in many ways, and I thank you each for the special ways you have all been a part of my life. To the special prayer partners I have been blessed to have, Pamela BingPerry, Lenay Edwards, and Belinda Ellis, and to Linda Ortega, for being a prayer mentor to me! I thank each of you for being not only prayer partners with me, but also for being prayer warriors with me! I love each of you!

To Elynda Bedney, I thank you for your persistence and constant search of financial resources for me that was the sole reason I was able to continue on with and finish my degree. I can never express just how much I appreciate and thank you for all that you did for me! To Karen Benjamin and Anna Piskozub, I thank you for all of the support you have given me as a student, and for your assistance to me with everything that I have needed over the years! Thank you for always being on top of things, and for keeping me on track! Without both of you, this Department would be lost!

To Dr. Nadia Nosworthy, I thank you for your constant care for your students, and for your openness and love to each of us! You truly make a difference in the lives of your students! I will never forget all that you did for me, and for the special accommodations for me when I was in the wheelchair!

Finally, I wish to thank the 50 participants of the quantitative portion of my research study and the 3 participants of the qualitative portion of the study. Without you this study would not have come together as a finished product. 


\section{CHAPTER 1}

\section{INTRODUCTION}

\section{General Introduction}

This study examined Posttraumatic Stress Disorder (PTSD) in private sector Emergency Medical Technicians (EMT's) of all levels: EMT-Basics (EMT-B's); EMT-Intermediates (EMTI's); and EMT-Paramedics (EMT-P's), and how they coped with trauma, how their emotional intelligence impacted their levels of PTSD, whether their attachment styles impacted the presence and/or expression of PTSD, and how their levels of resilience impacted their levels of PTSD. As a result of personal experience in the field as an EMT, as well as from the observation from both this researcher's personal life and from the observation from others' in the field, qualitative exploration became a focus of the study as well. This portion of the study examined the lived experience of private sector EMT's e and whether that experience was similar to public sector EMT's. It also examined factors that were unique to private sector EMT's and the possible outcome of those factors on PTSD symptom expression in this population of first responders.

There is a long-standing history of research related to PTSD, with initial research being performed on military populations, and on military veterans (Morrison, 2014). It was initially believed that only military individuals could experience PTSD, but as research has continued in the field, it has become clear that anyone who has been exposed to a traumatic event or who has become aware of someone who has, can suffer from PTSD (Beckman, 2015; Dass-Brailsford, 2010; Stellman, Smith, Katz, Sharma, Charney, Herbert,. . . Southwick, 2008; Long, Meyer, \& Jacobs, 2007; Smith, 2007; VanDeusen \& Way, 2006; Simons, Gaher, Jacobs, Meyer, \& Johnson-Jimenez, 2005; McCaslin, Jacobs, Meyer, Johnson-Jiminez, Metzler, \& Marmar, 2005; 
Fullerton, Ursano, \& Wang, 2004). Because we now know that military populations are not the only populations experiencing PTSD, it has been important to identify other individuals who may be experiencing the debilitating symptoms associated with PTSD so that appropriate services may be provided. As research has progressed, multiple other populations of individuals came to be acknowledged, including first responders such as police, firefighters, emergency medical services (EMS) workers, and those exposed to traumatic events such as floods, tsunamis, earthquakes, 9/11, and other catastrophic events PTSD (Beckman, 2015; Dass-Brailsford, 2010; Stellman, Smith, Katz, Sharma, Charney, Herbert,. . . Southwick, 2008; Long, Meyer, \& Jacobs, 2007; Smith, 2007; VanDeusen \& Way, 2006; Simons, Gaher, Jacobs, Meyer, \& JohnsonJimenez, 2005; McCaslin, Jacobs, Meyer, Johnson-Jiminez, Metzler, \& Marmar, 2005; Fullerton, Ursano, \& Wang, 2004).

\section{PTSD Criteria and Definitions}

The Diagnostic and Statistical Manual of Mental Disorders (DSM-5) classifies PTSD as a “Trauma and Stressor-Related Disorders" (APA, 2013, p. 265) which is designated for disorders that are inclusive of "disorders in which exposure to a traumatic or stressful event is listed explicitly as a diagnostic criterion" (p. 265). Criteria for diagnosis includes "witnessing or directly experiencing a traumatic event, learning about the traumatic events that happened to a close friend or family member, or repeatedly experiencing the aversive effects of a traumatic event; the presence of intrusion symptoms following the association with the traumatic event; a persistent and direct avoidance of stimuli that is associated with the traumatic event" (p. 271); “experiencing negative moods and altered mental cognitions, a change in one's arousal state and reactivity that are associated with the traumatic event; the duration of these states and symptoms for a period greater than one month; disturbance, not associated with the psychological effects of 
a substance or another medical condition" (p. 272), that "significantly impairs the individual or creates distress in one or more areas of functioning in their life" (pp. 271, 272). Specifiers for diagnosis may include "dissociative symptoms, a sense of derealization, and/or a sense of depersonalization, and also need to specify whether the expression of PTSD symptomatology is immediate or delayed" (p. 272). Bomyea, Risbrough, and Lang (2012) define PTSD as a "pathological response to a traumatic event such as combat, natural disasters, or physical or sexual assault" (p. 2), while Kremen, Koenen, Afari, and Lyons (2012) others define it as "an outcome that is defined by one's response to an environmental event" (p. 2). A third class of researchers use DSM criteria to define PTSD referring to it as "an anxiety disorder that manifests in response to a traumatic event that involves an actual or perceived threat of death or injury" (Mišić et al, 2016, p. 1; Acheson, Gresack, \& Risbrough, 2012; APA, 2000 as cited in Mišić et al, 2016). It is this final definition that was be the foundation of this research, and the one that was used throughout this dissertation by this researcher.

\section{Research on PTSD}

\section{Brain Structures}

Research on individuals with PTSD demonstrate that multiple brain structures may be affected. Identified brain structures that have been shown to have an association with the expression and exacerbation of PTSD symptomatology include the hippocampus, amygdala, insula, thalamus, HPA (hypothalamic-pituitary-adrenal axis), posterior parietal cortex, temporal lobes, cingulate cortex, and the prefrontal cortex (Tyrka, Burgers, Philip, Price, \& Carpenter, 2013; Antunes-Alves \& Comeau, 2014; Marin, Camprodon, Dougherty, \& Milad, 2014; Brenner, 2011; Lanius, Bluhm, \& Frewen, 2011). 


\section{Physical and Mental Health}

Additionally, multiple health related risks have also been associated with PTSD. These include an increased risk of Coronary Heart Disease (Caska, Smith, Renshaw, Allen, Uchino, \& Carlisle, 2014; Kubzansky, Koenen, Jones, \& Eaton, 2009), greater job loss (Brackbill, Hadler, DiGrande, Ekenga, Farfel, Friedman, Perlman, Stellman, Walker, Wu, Yu, \& Thorpe, 2009), increased rates of unemployment (Galovski \& Lyons, 2004), persistent and long-term mental health conditions (Brackbill, Hadler, DiGrande, Ekenga, Farfel, Friedman, Perlman, Stellman, Walker, Wu, Yu, \& Thorpe, 2009), increased marital distress and difficulties in intimate relationships (Caska, Smith, Renshaw, Allen, Uchino, \& Carlisle, 2014), and marital instability (Galovski \& Lyons, 2004). Family adjustment is also impacted, as well as marital intimacy, and violence against women has been shown to be at much higher rates in men who have been diagnosed with PTSD (Monson, Taft, \& Fredman, 2009). Rates of divorce increase exponentially, as does the risk of developing PTSD and Secondary Traumatic Stress (STS) to wives of men with PTSD (Monson, Taft, \& Fredman, 2009). Increased rates of depression (Lanconi, S., Vigouroux, Lafuente, \& Chabrol, 2017; Galor, \& Hentschel, 2012; Wagner \& Martin, 2012), anxiety (Wagner \& Martin, 2012), and other mental health outcomes have also been identified by researchers. The term, Secondary Trauma Stress (STS) was coined by Figley (1983), and is defined as trauma that is experienced as a result of violent trauma that has been experienced by a loved one (Dekel \& Monson, 2010). Although STS is a well-established construct clinically, clinicians still confuse it with other stress-related diagnoses, such as compassion fatigue and burnout (Rzeszutek, Partyka, \& Golab, 2015). STS demonstrates the same expression of symptoms as PTSD, but its onset is due to the individual experiencing the traumatic events vicariously as a result of interactions with those who were personally affected 
by the traumatic events (Figley, 1995; Shoji, Jock, Cieslak, Zukowska, Luszczynska, \& Benight, 2014).

\section{Risk Factors for PTSD}

According to a meta-analysis performed by Acheson, Gresack, and Risbrough (2012), risk factors for developing PTSD include genetic and epigenetic factors, such as early life stressors, that contribute to an increase in the expression of stress responses in adulthood (McGowan et al., 2009 as cited in Acheson, Gresack, \& Risbrough, 2012). Included in this are childhood abuse (Tyrka et al, 2013) and being female (Malta, 2012; Schmidt, Kaltwasser, \& Wotjak, 2013; Tyrka et al, 2013), previous exposure to trauma (Tyrka et al, 2013, and having a family history of psychopathology (Brewin, Dalgleish, \& Joseph, 1996 as cited in Bomyea, Risbrough, \& Lang, 2012; Ozer, Best, Lipsey, \& Weiss, 2003 as cited in Bomyea, Risbrough, \& Lang, 2012; Tyrka et al, 2013).

Studies have documented that female are at a greater risk for PTSD than are males (Kubzansky, Koenen, Jones, \& Eaton, 2009; Tolin \& Foa, 2006). Dekel and Monson (2010) assert that the increased risk of PTSD for women may possibly be due to the greater sense of caregiver burden that is expected of women, than is expected of men, as well as due to the increased empathy that women demonstrate than men (Zaki, 2014).

\section{Statement of the Problem}

It is currently unclear how private sector EMTs experience PTSD, and if their traumatic experiences fall under a set of considerations that one would find with other populations of first responders such as firefighters and police officers. Because private sector EMTs are most often the first line of defense along with other entities such as police officers and firefighters, their exposure to traumatic events is as severe as other first line responders. However, news agencies 
and the public themselves do not recognize private sector EMTs with the same vigor or accolade that police officers and firefighters are recognized, nor are the risks to life, health, and mental health outcomes recognized in this population of individuals. This lack of recognition may be seen in the literature as reflected by the lack of research in populations of private sector EMTs. It may also be seen in the statements of private sector EMTs who have voiced this sentiment to this researcher, thereby prompting the current research study. Because of the lack of research in private sector EMTs, it is important to identify how PTSD may be experienced by this population and to examine what unique risk factors may be present so that adequate and appropriate treatment options may be established. Within this lies the need for therapists to be aware of the unique needs of private sector EMTs, as well as the need for individuals such as myself, with training and experience in both the EMS field and in the mental health field to provide information and training to those without this dual perspective.

It is often difficult for the general public to understand the difference between the public and private sector of EMTs because they both do the same type of work. One difference between the two is that the public sector of EMTs are funded through taxpayer money as well as through fees charged to the patients who use those services (Calams, 2017). Additionally, taxpayers pay for public ambulance services, usually provided by the fire department, whether those services are used or not (Calams, 2017). For example, taxpayers are charged a specific amount in their city taxes for these services, just to have them available. Another difference is that the pay is significantly higher for public sector EMTs, which are usually fire fighters, since fire fighters are paid hazard pay for fire calls. Although EMS calls are not fire calls, there is no differentiation in pay for non-hazard calls. Thirdly, public sector EMTs are typically viewed as having greater training and experience, when certification requirements are most often the same. 
In opposition, the private sector is privately funded, with the ambulance service being funded solely by the calls received by private ambulance companies (Calams, 2017); taxpayer money does not fund private ambulance companies. In this, then, the only services billed for are when patients use these services (Calams, 2017). Additionally, private sector EMTs are paid much lower wages, and are often seen as "ambulance drivers" when they are performing the same, and often more, work than fire EMTs while on calls. While both private and public sector EMTs respond to traumatic calls; however, because of how private sector EMTs are viewed by the public, there is a paucity of studies performed on private sector EMTs, and the literature is scant in terms of how private sector EMTs respond to and experience PTSD.

\section{Coping Skills}

It is clear that coping skills have a significant correlation with PTSD expression and severity. Multiple studies with multiple populations have demonstrated the impact of those who utilize avoidant coping as a means of dealing with the stressful event. A 2012 study examined refugees that had settled in the Netherlands following war, persecution, and violence, with the study outcomes again demonstrating a link between PTSD severity and avoidant coping (Huijts, Kleijn, can Emmerik, Noordhof, \& Smith, 2012). These same results were found in a study on Israeli veterans who had been diagnosed with PTSD (Galor, \& Hentschel, 2012). The final study published in 2012 was performed on veterans who had been exposed to traumatic situations, and who were receiving mental health care services through the Veterans Administration (VA). While this study identified emotional avoidance to be associated with PTSD symptom severity, the researchers also found that low levels of emotional expression was also associated with PTSD symptom severity (Hassija, Luterek, Naragon-Gainey, Moore, \& Simpson, 2012). 
Avoidant coping was the only resulting factor that demonstrated a significant association with changes in PTSD symptom severity over a 1-year period among veterans who had served in combat zones (Lee, Possemato, \& Ouimette, 2017). Finally, when examining the ability to adjust following a traumatic event, Slanbekova, Chung, Abildina, Sabirova, Kapbasova, and Karipbaev (2017) identified emotion-focused coping and the inability to manage one's emotions to be correlated with difficulties in adjustment, whereas the use of problem-focused coping and being unable to manage one's emotions were correlated with greater symptom severity in couples following a divorce.

Because coping has been associated with PTSD symptom expression and severity, it was important to include in this study. Very little research has examined coping skills in field EMTs. With field EMTs being a primary front-line response system, this leaves field EMTs as a population at great risk for the development of PTSD symptom expression due to the knowledge that is existent for other similar populations. The lack of research for this population also leaves a great need for treatment options and resources to be examined and instituted for the population of first responders.

For this study, this researcher used the description of coping as proposed by Carver and Scheier (1994) is stated to be the manner in which people react to and cope with stressors and difficulties in their lives, and the tools that they utilize in response or reaction to those stressors and while under stress (Carver \& Scheier, 1994). His measure, the Brief COPE, identified four coping subscales: Use of Emotional Social Support; Use of Problem Solving; Avoidance Coping; and Use of Positive Thinking. It was hypothesized that EMTs demonstrating avoidant coping would show greater levels of PTSD symptom severity. 


\section{Emotional intelligence}

As with coping strategies and skills, emotional intelligence has been studied in many populations, with the consensus being that individuals demonstrating greater emotional intelligence also showing lower levels of PTSD severity and expression. For example, these findings were exhibited in a study on adolescent refugees from the Middle East (Ghazali, 2014), young children experiencing mental health issues and trauma, including those expressing suicidal ideation (Bodzy, Barreto, Swenson, Liguori, \& Costea, 2016), youth exposed to community violence (Stokes \& Jackson, 2014), prostate and bladder cancer patients (Smith, Turner, Pati, Petrides, Sevdalis, \& Green, 2012), those diagnosed with borderline personality disorder (Gaher, Hofman, Simons, \& Hunsaker, 2013), learning disabled individuals (Chantrell, 2009), individuals with HIV/AIDS (O’Cleirigh, Ironson, Fletcher, \& Schneiderman, 2008), divorced couples (Slanbekova, Chung, Abildina, Sabirova, Kapbasova, \& Karipbaev, 2017), and adults with previous traumatic experiences (Benoit, Bouthillier, Moss, Rousseau, \& Brunet, 2010). It was proposed in each of these studies that individuals with greater levels of emotional intelligence would demonstrate lower levels of PTSD symptom severity, which was found to be the case in each of the studies, without exception.

Several studies identified emotional intelligence as a mediating factor between trauma exposure and PTSD symptom severity (Gaher, Hofman, Simons, \& Hunsaker, 2013; Benoit, Bouthillier, Moss, Rousseau, \& Brunet, 2010; O’Cleirigh, Ironson, Fletcher, \& Schneiderman, 2008), and one study identifying emotional intelligence as a moderating factor between emotional expression and posttraumatic growth (Linley, Felus, Gillett, \& Joseph, 2011). Linley et al. (2011) reported that emotional intelligence was not a significant factor for women in the expression of emotions and posttraumatic growth, but that for men, those with lower emotional 
intelligence/emotional expression was demonstrated to be a detriment to posttraumatic growth. This ability to understand and the ability to use emotion were found to be the two most significant factors in PTSD symptom expression and emotional intelligence in Ghazali's (2014) study. This would be an important factor, since the ability to understand and use emotion could quite possibly impact the outcome of exposure to traumatic events, a common and expected aspect of field work for EMTs, and the development of problematic trauma response and symptom expression.

However, very little research has been performed on the relationship between emotional intelligence and traumatic stress in EMTs, with one research article resulting from a literature search on emotional intelligence and emergency medical services personnel. Wagner and Martin (2012) examined emotional intelligence and proactive coping on mental health in firefighters, with their results indicating a negative relationship between emotional intelligence and traumatic stress symptomatology. While this study came the closest to examining emotional intelligence and PTSD in field EMS workers, the study looked solely at firefighters, rather than EMTs. The lack of research on field EMTs remains a limited but an important population due to the fact that these individuals are the first line responders in traumatic incidents, both in the home as well as on the road. Based on the demonstrated differences in the outcome of emotional intelligence and PTSD symptom expression between men and women, examining whether these differences hold up in field EMTs as well are important so that resulting outcomes may be used to determine treatment needs for this population.

For this study, the definition of emotional intelligence as proposed by Mayer and Salovey (1997) was used, which proposes that emotional intelligence is comprised of the ability to understand emotion, ability to utilize one's own emotion to assist in facilitating the thinking 
process, and the ability to manage both one's own emotion and others' emotions, including the ability to perceive and express emotion. The measure that was used to evaluate for emotional intelligence was the Schutte Emotional Intelligence Scale (SEIS), which is also known as the Schutte Self-Report Emotional Intelligence Test (SSEIT) and the Self-Report Emotional Intelligence Test (SREIT), which identifies three aspects of emotional intelligence: "how one appraises and expresses emotion, the individual's ability to regulate emotion, and how emotion is utilized in the individual's life" (Consortium for Research on Emotional Intelligence in Organizations, 2017, p. 160). The model created by Schutte, Malouff, and Bhullar "proposed that emotional intelligence consists of appraisal of emotion in the self and others, expression of emotion, regulation of emotion in the self and others, and utilization of emotion in solving problems (Schutte, Malouff. \& Bhullar, 2009, p. 119). Along with others (Petrides \& Furnham, 2000; Saklofske, Austin, \& Minski. 2003), Ciarrochi, Deane, and Anderson (2002) performed factor analytic studies, which resulted in four subscales of emotional intelligence: Managing Own Emotions; Managing Others' Emotions; Perception of Emotion; and Utilization of Emotion. It was hypothesized that the inability to perceive, manage, or utilize one's own emotions would be associated with greater levels of PTSD expression.

\section{Attachment}

Attachment has also been a well-researched area, although not in relation to private sector EMTs. Emotional attachment was proposed by Bowlby $(1973,1982)$ as the style of behavior by which humans connect with other human beings (Mikulincer, Solomon, Shaver, and Ein-Dor, 2014); with the two primary styles of attachment being anxious attachment and avoidance attachment (Brennan, Clark, \& Shaver, 1998). Anxious attachment has been described as "the degree to which a person worries that others will not be available in times of need" (Mikulincer, 
Solomon, Shaver, and Ein-Dor, 2014, p. 209), and avoidant attachment has been described as "the extent to which a person inhibits support-seeking and relies on him/herself as the sole source of safety" (Mikulincer, Solomon, Shaver, and Ein-Dor, 2014, p. 209). Brennan, Clark, and Shaver (1998) assert that those who score low on both of these attachment dimension may be said to be securely attached, whereas those who score high on both of these dimensions exhibit insecure attachment.

Ainsworth was the first to propose attachment styles or patterns, in which she identified three primary patterns: Secure Attachment; Insecure Avoidant Attachment; and Insecure Resistant (Ambivalent) Attachment (Reddan, 2015; Ainsworth, Blehar, Waters, \& Wall, 1978; Ainsworth, 1970). Charles S. Carver (1997a) created The Measure of Attachment Qualities (MAQ), which measures four subscales of attachment: Avoidant Attachment, Ambivalent-Worry (Ambivalent) Attachment, and Ambivalent-Merger (Anxious) Attachment, and Secure/Insecure Attachment.

Attachment style has been suggested to be related to the development of trauma-related symptoms, with child abuse being attributed to result in insecure attachment styles (Alexander, 1992; Muller, Sicoli, \& Lemieux, 2000), as has family functioning and maternal depression (O'Connor, \& Elklit, 2008). Lower levels of attachment have been shown to be associated with greater levels of psychological symptom expression (Elwood \& Williams, 2007). Elwood and Williams (2007) also found associations between PTSD-related cognitions, which include "negative cognitions about the self, negative cognitions about the world, and self-blame for the trauma," (Elwood \& Williams, 2007, p. 1190) and the development of both PTSD and depressive symptoms. Individuals with attachment avoidance and attachment anxiety demonstrated greater levels of PTSD in a study by Busuito, Huth-Bocks, and Puro (2014) as well. Clark and Owens 
(2012) found the same relationship between anxious attachment in romantic relationships and PTSD symptom severity in U.S. veterans of Iraq and Afghanistan. A meta-analysis performed by Woodhouse, Ayers, and Field (2015) once again confirmed the association between insecure attachment and PTSD symptoms.

Multiple other studies with various populations demonstrate the same connection between anxious and insecure attachment and PTSD symptomatology, including civilians in Southern Israel exposed to terrorist attacks (Besser, Neria, \& Haynes, 2009), women with gynecological cancer (Holt, Jensen, Hansen, Elklit, \& Mogensen, 2016), individuals with chronic whiplash-associated disorder (Andersen, Elklit, \& Vase, 2011), Vietnam combat veterans (Nye, Katzman, Bell, Kilpatrick, Brainard, \& Haaland, 2008), university staff and students (Arikan, Stopa, Carnelley, \& Karl, 2016), civilians exposed to the Israel-Gaza war in 2009 (Avi, \& Yuval, 2010), security guards (Bogaerts, Daalder, Van Der Knaap, Kunst, \& Buschman, 2009), female healthcare workers in a longitudinal study on violence and health (La Flair, Bradshaw, Mendelson, \& Campbell, 2015), and school children following a school-shooting trauma in Finland (Turunen, Haravouri, Punamaki, Suomalainen, \& Marttunen, 2014). Ein-Dor, Doron, Mikulincer, Solomon, \& Shaver (2010) found that this connection was significant enough that it affected not only the individual exposed to the traumatic event, but to their romantic partner as well in relation to whether that individual developed Secondary Stress Trauma (STS) or not. Benoit, Bouthillier, Moss, Rousseau, and Brunet (2010) propose that it is the individual's attachment, whether that be insecure or secure, that comes into play when an individual is exposed to a threatening or stressful situation.

Taking into consideration the importance of attachment in the development and sustenance of PTSD symptoms, it is of great value to examine how attachment may factor in as a 
variable in the development of PTSD in field EMTs, and to identify whether attachment serves as a treatment need in this population as well. In this study, the MAQ was used for identifying attachment styles. It was hypothesized that individuals with Avoidant Attachment, AmbivalentWorry (Ambivalent) Attachment, Ambivalent-Merger (Anxious) Attachment, and Insecure Attachment would demonstrate greater levels of PTSD symptoms.

\section{Resilience}

Resilience is the final variable chosen for this study, and is one that has been researched along with coping in many studies examining PTSD. Resilience has been defined and conceptualized in several different ways. Oginska-Bulik and Kobylarczyk (2014) make the distinction between resilience and resiliency, proposing that resiliency is more of a characteristic or personality trait that individuals possess, and that resilience is more of a process. This has been proposed by other researchers as well (Yehuda \& Flory, 2007), stemming from the debate that is present in most research on human behavior; whether the variable being studies is a state or trait, or a combination of both. Resilience has also been defined as the ability to bounce back after a traumatic event (Yehuda \& Flory, 2007), as being a "concept of personal hardiness" (Kuiper, 2012, p. 475), or the "individual ability to separate from unpleasant life events" (Oginska-Bulik \& Kobylarczyk, 2015, p. 708). Still others propose that resilience is the ability on the part of first responders to withstand trauma (Chang \& Taormina, 2011), and "the ability to rebound from and positively adapt to significant stressors: (Sinclair \& Wallston, 2004, p. 94).

Research on resilience has focused on both the effects of resilience in different situations and circumstances, as well as with regard to the components that make up resiliency. Yehuda and Flory (2007) assert that prior to the conceptualization of PTSD as a diagnosis in the DSMIII, resiliency was considered to be a fundamental component of the individual, with those who 
were considered to be "resilient" demonstrating the ability to bounce back after traumatic and adverse life experiences, while those who didn't being considered to be lacking in resiliency. This lack of resiliency, therefore, was considered to be a character flaw for which people were severely stigmatized (Yehuda \& Flory, 2007). Once PTSD was established as a recognized diagnosis in 1980, the door was opened for examination of the variables that factored into the expression and severity of its symptoms (Yehuda \& Flory, 2007).

Some of the questions that have been examined in relation to resiliency over the years have included the examination of resiliency as a psychopathological component and resiliency as a component of vulnerability (Yehuda \& Flory, 2007, p. 439). Richardson (2002) proposed a metatheory on resilience in which it was proposed that the research on resilience has followed three primary avenues: 1) the process of resiliency as developed by those individuals who have faced adversity; 2) the characteristics of those individuals; and 3) the cognitive mechanisms that accompany the development of resiliency for the individual. Some of the factors that have been examined have included an examination of the type of trauma exposure, prior history of trauma exposure, personality characteristics, genetics and neurobiological factors, gender, age, childhood factors, affect regulation, ego defenses, cognitive factors, and social support systems (Wilson, 1995; Zeidner \& Endler, 1996; Zuckerman, 1999; Frederickson, 2002; Agaibi, 2003; Schore, 2003; Southwick, Morgan, Vythilingham, Krystal, \& Charney, 2004).

Resilience has been associated with post-traumatic growth (Vera, 2013; Oginska-Bulik and Kobylarczyk, 2014), with positive emotions (Frederickson, 1998; Frederickson, 2001), greater levels of physical wellness (Chang \& Taormina, 2011), higher levels of satisfaction with personal relationships, medical care, and community life (Chang \& Taormina, 2011), and lower levels of burnout (Chang \& Taormina, 2011). It has also been found to be a contributing factor 
in the determination of perceived levels of stress (Mroz, 2015), and it has been found to serve as a buffer against depressive symptoms (Sinclair \& Wallston, 2004).

According to a review performed by Agaibi and Wilson (2005), seven factors have been identified that appear to be associated with the development of resilience as reported by Raphael (1993) and Wilson (1995). These were:

(a) locus of control (i.e., a sense of efficacy and determination, (b) self-disclosure of the trauma experience to significant others, (c) a sense of group identify and sense of self as a positive survivor, (d) the perception of personal and social resources to aid in coping in the posttrauma recovery environment, (e) altruistic or prosocial behaviors, (f) the capacity to find meaning in the traumatic experience and left afterward, and $(\mathrm{g})$ connection, bonding, and social interaction within a significant community of friends and fellow survivors. (Agaibi \& Wilson, 2005, p. 203)

Based on the research outcomes related to resilience and the significance that has been demonstrated for multiple populations of individuals who have experienced trauma, it is imperative that research be initiated for field EMTs as well due to the increased possibility for PTSD as a result of the nature of their work. Resilience, is defined by Connor and Davidson (2003) as "the personal qualities that enable one to thrive in the face of adversity" (p. 76). The Connor Davidson - Resilience Scale was developed because the researchers felt that there was a need for "a stronger presence in the field of PTSD assessment" among the measures that were available, and that despite the fact that resiliency measures existed, "they had largely failed to penetrate into the world of clinical practice" (http://www.connordavidsonresiliencescale.com/about.php). Therefore, the CD-RISC was developed to meet this need. There are three versions of the Connor-Davidson - Resilience Scale (CD-RISC). There is a 25item CD-RISC (CD-RISC 25), and two briefer versions; the 10 item (CD-RISC 10) and the two item (CD-RISC 2) scales (http://www.connordavidson-resiliencescale.com/about.php). The CD- 
RISC 10 was the measure used for this study. It was hypothesized that EMTs with lower resiliency scores would show greater levels of PTSD symptom severity.

\section{The Purpose of the Study}

The purpose of this study was to examine the relationship between coping skills, emotional intelligence, attachment, and resilience on PTSD symptoms in private sector Emergency Medical Technicians (EMTs) workers.

\section{Four Factor Model}

As discussed above, the four variables chosen for this study; coping, emotional intelligence, attachment, and resiliency have all been demonstrated to impact PTSD symptom expression and symptom severity in multiple populations. Post-traumatic factors that may increase the likelihood of developing PTSD may include factors such as short periods of time allocated by others to recover from the event, dissatisfaction with one's support system, or the lack of social support outside of the incident-related situation, incident-related physical injuries, depressive symptoms, and subsequent negative life events (Marchand, Boyer, Nadeau, BeaulieuPrévost, \& Martin, 2015). The study by Marchand et al (2015) did support the fact that risk factors can be predictive of PTSD development; however, strong support factors can prevent this as well. The fact that first responders experience all of these factors is important in understanding both the nature of the job, but, also how avoidance plays a role in treatment resistance and rejection (Marchand, et al, 2015). However, as previously stated, factors related to private sector EMS workers are much less understood due to the lack of research on this particular population.

In this then, it was important to understand not only the differential factors in PTSD, but also the role that coping, emotional intelligence, attachment, and resilience play a role in those 
who are exposed to traumatic events, and for whom the societal acknowledgement and recognition is lacking. Further, it wa`s important to understand how this may prevent the seeking of treatment services, and whether appropriate treatment services are even available to this population of first responders.

It was hypothesized that as inadequate and ineffective coping skills are maintained, as individuals fail to demonstrate effective levels of emotional intelligence and emotional intelligence, and as individuals experience ineffective levels of attachment, along with the lack of societal recognition and support, the more likely individuals are to experience PTSD symptoms, and to avoid treatment even when it is offered.

Because most of the research on PTSD in first responders on military veterans, police officers, and firefighters (including paramedics with the fire service) who present with symptoms of PTSD, and has focused very little on private sector EMTs who have been exposed to traumatic events and are presenting with the same symptoms of PTSD, most of the literature references these more recognized populations of first responders as the primary means of exposure to a traumatic event. This leaves the field open to the necessity of additional study with the private sector of EMTs that have been exposed to and have experienced traumatic events in much the same rate and manner as the more recognized of first responder populations. Without further studies related to the manner that trauma exposure in the private sector of first responders impact PTSD expression and symptom severity, it is impossible to effectively treat those within the private sector who are experiencing PTSD in whatever format that it is present in their lives.

\section{Theoretical Background and Assumptions}

The theoretical background and assumptions related to this research included the Biopsychosocial-Spiritual Model, Social Constructionism, and Narrative Theory. The 
Biopsychosocial-Spiritual Model asserts that an individual's biological, psychological, social, and spiritual factors all play a part in the physical and mental health of the individual, and that deviations from health are a result of an interruption to any one of these systems (Nietzel, Bernstein, Kramer, \& Milich, 2003). Social Constructionism asserts that meaning and reality is constructed through socially constructed ideas and societal practices (Gergen, 1994; Gergen, 2001). It is through societal dominant discourses that individual's find their meaning and the identities that they are expected to assume (Burr, 2004; Burr, 2001). Closely related to Social Constructionism is Narrative Theory, which proposes that it is through the stories that people tell and are told that shape their lives (Nichols \& Schwartz, 2004). Without the recognition of what those messages are, the individual is essentially at the mercy of the dominant society and discourse. It is only through the examination of each of these factors as the base for the individual's thoughts and actions that one may begin to deal with the components that play into the experience of PTSD. Further, societal expectations and rules play a significant factor in the functioning and actions of individuals directly as related to how one deals with trauma, and what is acceptable and what is not. In this, then, each of these theoretical assumptions are relevant to the study at hand.

\section{The Research Questions}

\section{Quantitative Research Questions}

Following were the research questions for the quantitative portion of this study:

1. What is the severity of PTSD among private sector EMTs?

2. Are there differences in the severity of PTSD among EMT-B's, EMT-I's, and EMTP's?

3. What coping strategies do private EMTs use? 
4. What are their levels of emotional intelligence, resilience, and attachment?

5. What is the nature of the relationship between PTSD severity and the following variables: emotional intelligence, coping skills, resiliency, and attachment?

\section{Qualitative Interview Questions}

The questions for the individual interviews during the qualitative portion of the study were as follows:

1. Describe your work environment as an EMT working in the private sector of the field.

2. What were some of the things that drew you to desire working in this field?

3. Describe what brought you to working in the private sector versus the public sector of the field.

4. In your opinion, what are the differences between working in the private versus the public sector of the field?

5. How does the EMS culture play a role in your experiences as a private sector EMT?

6. In your opinion, is there a difference in community or societal response to you as a private sector EMT versus a public sector EMT? If so, how does the public's response to your work in the private sector impact your work as a private sector EMT?

7. What advice would you give to someone who is considering working in the private sector of the field? 


\section{Qualitative Objective}

\section{Qualitative Objective}

The objective of the qualitative portion of this study was to identify the experiences of EMTs working in the private sector of the field to evaluate for three factors: 1) Whether there were perceived differences between working in the private versus the public sector of the field;

2) If private sector EMTs express similarities in the role EMS culture plays in their experiences as private sector EMTs; and 3) Whether private sector EMTs express perceived differences in community or societal response to their work as opposed to community or societal response to the work of public sector EMTs. Because the quantitative portion of the study addressed the component related to trauma through the examination of PTSD, questions related to trauma were not addressed in this section.

\section{Importance and Significance of the Study}

While PTSD has been a long-researched area, both in the medical and the mental health fields, research has primarily been focused on PTSD only as it relates to combat veterans and those directly and actively in military service, and to populations that have been recognized by society as impacted populations of first responders, such as police officers, firefighters, and paramedics employed in the public sector. The research has clearly documented how PTSD relates to these populations, but there has been a paucity of research related to PTSD in the private sector of first responders, as discussed above, with the severity of outcomes related to the outcomes as related to PTSD symptom expression, it was imperative that research on PTSD be expanded to examine PTSD in private sector EMTs in order to identify the differences between more recognized populations of first responders, and to identify the similarities that private sector first responders share with private sector first responders who experience PTSD. Finally, 
it was imperative to identify where the holes were with regard to treatment options for private sector EMTs so that effective, relevant, and adequate treatment options and services may be developed where services are identified to be lacking.

This research study sought to identify the severity of PTSD among private sector EMTs and how PTSD is expressed within this population. This included identifying any differences between the three EMT levels within the private sector, if any. It also sought to identify the types of coping strategies that private sector EMTs use, as well as what levels of emotional intelligence, resilience, and attachment were present within the private sector for EMTs. Finally, it sought to identify the nature of the relationship between each of these variables and PTSD. The goal of this information was not only to provide a baseline view of PTSD for private sector EMTs, but also to enable future research to evaluate differences and similarities between private sector and more community-based EMTs, such as those working for community and governmental organizations. To be included in that was the goal for therapist's to have the knowledge related to unique needs of those who present for services, as well as to assist with the education of the public regarding private sector EMT's.

The importance of this research was that it would further the knowledge in the field related to how private sector EMTs experience PTSD symptoms and PTSD symptom severity, and how this affects the maintenance of PTSD within that individual's life. It also assisted in recognizing this important population. Finally, this information provides guidance to assist psychologists, therapists, and social workers in identifying the impact of PTSD on private sector EMTs, and in providing appropriate treatment to these individuals. Finally, this research study opened up the field in PTSD by examining a previously unexplored population of first responders and identifying information specific to that population. With this study providing a 
general baseline into how PTSD is experienced by private sector EMTs, further research may be formulated and initiated relative to the findings of this study. In essence, the field of research related to PTSD has been opened up in a fuller and more representative way to those serving as first responders.

\section{Conceptual Framework}

Evaluating the literature on PTSD and PTSD symptom expression, it appeared that the diagnostic criteria for PTSD was likely the most accurate means by which to evaluate for the risk of PTSD in those exposed through the nature of their work to traumatic events. Therefore, it was conceptualized that using instruments which evaluate for those variables that are seen in the clinical setting as the expression of PTSD should prove to be a very effective means of identifying life style factors and areas of risk in an individual's life that could possibly lead to the expression of symptoms that would indicate the need for treatment, or at the very least, to identify individuals who may be at risk for PTSD in order to provide work-related programs to address those needs in EMTs that may be at risk. The conceptual framework for this may be seen below in Figure 1: 
Figure 1: Conceptual Framework

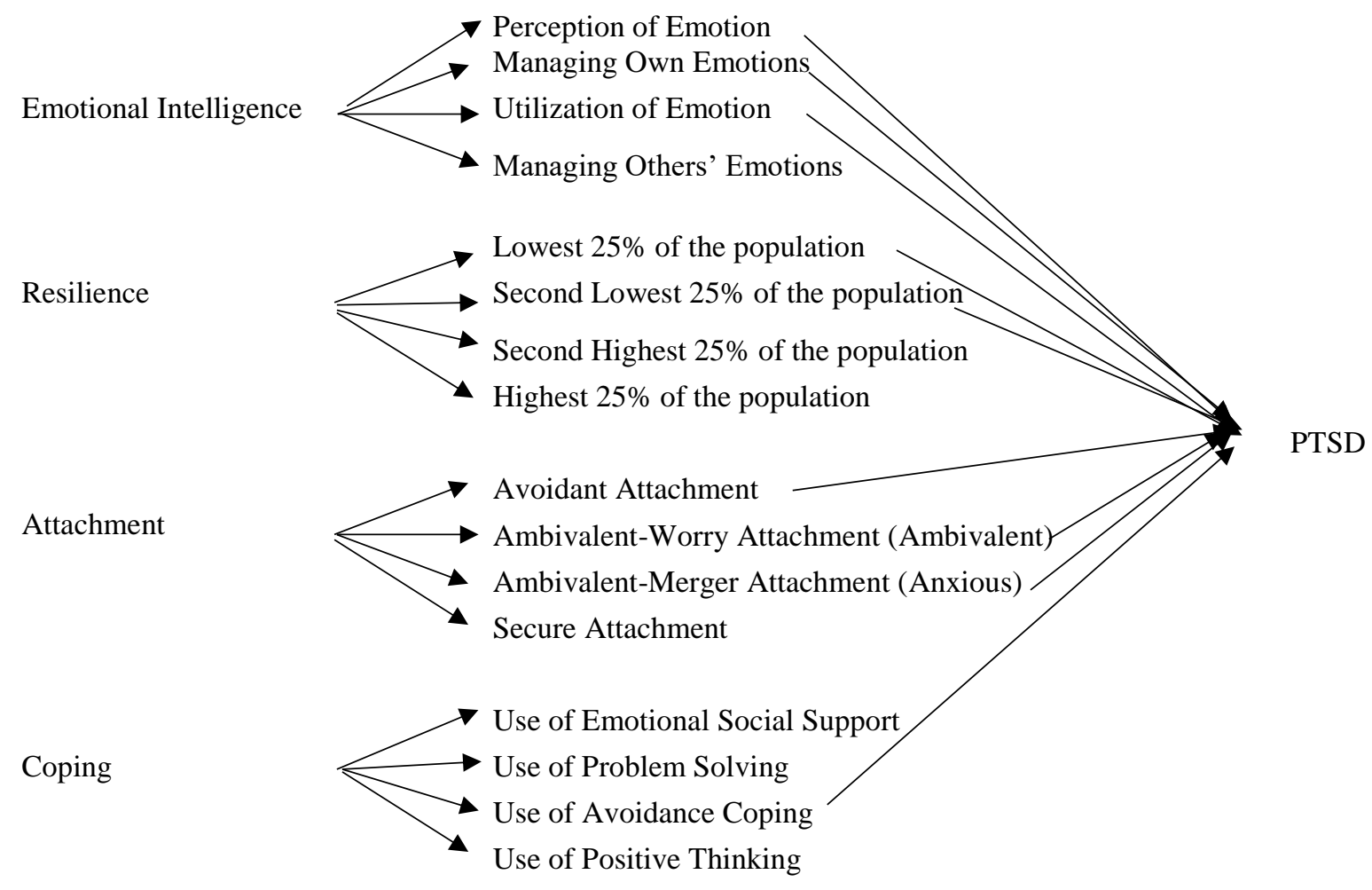

This framework asserted that the diagnostic criteria for PTSD was most likely to be seen through the examination of emotional intelligence, resilience, attach, and coping, as these are the variables that most closely align with the diagnostic criteria for PTSD. Emotional intelligence was likely to be seen through the use of the inability manage one's own emotions, the inability to perceive emotion in the self and others, and the inability to utilize emotion, which would then be identified by resulting in meeting a portion of the PTSD criteria; Resilience would be seen in those who have a lower level of resilience, thereby resulting in meeting a portion of the PTSD criteria; Attachment would be identified by the use of either avoidant, ambivalent, or anxious coping, again, meeting a portion of the PTSD criteria; and Coping that is avoidant would be the style that would meet a portion of the PTSD criteria. 


\section{Limitations and Delimitations}

\section{Delimitations}

Inclusion and exclusion criteria were identified for variables related to the search for literature. Eligible participants were comprised of: 1) individuals who work in the Emergency Medical Services field as a private sector EMT; 2) a private sector field EMT who has been exposed to traumatic events as a result of their job description and working conditions; or 3) an individual who has worked in the private sector as an EMT within the previous 5 years, and who has been exposed to traumatic events as a result of their job description and working conditions. Exclusion criteria included 1) individuals who are first responders, but who have never worked in the private sector of the EMS field; 2) individuals who are first responders and have worked in the private sector of the EMS field, but not within the previous 5 years; and 3) individuals who are first responders, but do not currently, nor have they ever, worked in the private sector.

\section{Limitations}

The limitations of this study include the ability of the data to be generalized only to private sector EMTs. In this, by including only private sector EMTs in this study, and by examining only those EMTs representing the private sector of first responders, the results are specific solely to that population. While implications for future research may be formulated from the results, implications for other populations of first responders will not be able to be made from study results.

\section{Definitions of Terms}

\section{Post-Traumatic Stress Disorder}

The Diagnostic and Statistical Manual of Mental Disorders (DSM-5) classifies PTSD as a "Trauma and Stressor-Related Disorders" (APA, 2013, p. 265) which is designated for disorders 
that are inclusive of "disorders in which exposure to a traumatic or stressful event is listed explicitly as a diagnostic criterion" (p. 265). Criteria for diagnosis includes "witnessing or directly experiencing a traumatic event, learning about the traumatic events that happened to a close friend or family member, or repeatedly experiencing the aversive effects of a traumatic event; the presence of intrusion symptoms following the association with the traumatic event; a persistent and direct avoidance of stimuli that is associated with the traumatic event" (p. 271); “experiencing negative moods and altered mental cognitions, a change in one's arousal state and reactivity that are associated with the traumatic event; the duration of these states and symptoms for a period greater than one month; disturbance, not associated with the psychological effects of a substance or another medical condition" (p. 272), that "significantly impairs the individual or creates distress in one or more areas of functioning in their life" (pp. 271, 272). Specifiers for diagnosis may include "dissociative symptoms, a sense of derealization, and/or a sense of depersonalization, and also need to specify whether the expression of PTSD symptomatology is immediate or delayed" (p. 272). Bomyea, Risbrough, and Lang (2012) define PTSD as a "pathological response to a traumatic event such as combat, natural disasters, or physical or sexual assault" (p. 2), while Kremen, Koenen, Afari, and Lyons (2012) others define it as "an outcome that is defined by one's response to an environmental event" (p. 2). A third class of researchers use DSM criteria to define PTSD referring to it as "an anxiety disorder that manifests in response to a traumatic event that involves an actual or perceived threat of death or injury" (Mišić et al, 2016, p. 1; Acheson, Gresack, \& Risbrough, 2012; APA, 2000 as cited in Mišić et al, 2016). 


\section{Coping}

Coping has been defined as the behavioral or cognitive efforts that are used by an individual to manage situations that are appraised as stressful (Prati, Pietrantoni, \& Cicognani, 2011). It has also been defined by Carver and Scheier (1994) as the manner in which people react to and cope with stressors and difficulties in their lives, and the tools that they utilize in response or reaction to those stressors and while under stress (Carver \& Scheier, 1994).

\section{Emotional intelligence}

Emotional intelligence has been defined as the ability for one to understand their own emotions and the emotions of others, having the ability to discriminate between emotions and feelings, and having the ability to monitor one's own emotions (Brackett, \& Geher, 2006). Others have conceptualized emotional intelligence as encompassing the emotional regulation process, which utilizes the use of cognitive, emotional, and behavioral strategies in order to regulate that process (Benoit, Bouthillier, Moss, Rousseau, \& Brunet, 2010). Still others identify emotional intelligence as including the perception and expression of emotion, the ability to understand emotion, being able to utilize one's own emotion to assist in facilitating the thinking process, and being able to manage both one's own emotion and others' emotions (Mayer \& Salovey, 1997). There are still others who view emotional intelligence as including not only the awareness of one's own and others' emotions, the ability to discriminate and regulate one's and others' emotions, and the ability to use emotions to facilitate thought (Matthews, Zeidner, \& Roberts, 2002), but also the ability to remain motivated and persist in spite of frustration, to delay gratification by controlling one's impulses, to appraise others' emotions accurately, to hold out hope and be able to enhance one's own life through the use of effective emotion regulation, 
and to be able to keep from allowing one's emotions from "swamping the ability to think" (Wagner \& Martin, 2012, p. 59).

\section{Attachment}

Attachment was proposed by Bowlby $(1973,1982)$ as the style of behavior by which

humans connect with other human beings (Mikulincer, Solomon, Shaver, and Ein-Dor, 2014).

\section{Resilience}

Resilience has been defined in multiple ways, including the ability to bounce back after a traumatic event (Yehuda \& Flory, 2007), a "concept of personal hardiness" (Kuiper, 2012, p. 475), the "individual ability to separate from unpleasant life events" (Oginska-Bulik \& Kobylarczyk, 2015, p. 708), the ability on the part of first responders to withstand trauma (Chang \& Taormina, 2011), and "the ability to rebound from and positively adapt to significant stressors: (Sinclair \& Wallston, 2004, p. 94).

\section{Emergency Medical Technician}

Emergency Medical Technician’s (EMTs) are providers of emergency medical treatment, most traditionally in a non-hospital setting, although EMTs are also utilized in Emergency Departments (ED’s) as well.

\section{Emergency Medical Technician - Basic's (EMT - B's)}

EMT-B's provide basic and intermediate emergency medical treatment, including basic life support measures and non-invasive procedures.

\section{Emergency Medical Technician - Intermediate's (EMT - I's)}

EMT-I's provide basic and intermediate emergency medical treatment, including basic life support measures and some invasive procedures such as starting IV's and intubating patients. 


\section{Emergency Medical Technician - Paramedic's (EMT - P's)}

EMT-P's provide basic and advanced emergency medical treatment, including basic and advanced life support such as reading labs, EKG's, and defibrillation procedures.

\section{Organization of the Study}

This chapter has introduced the beginnings of PTSD and noted the diagnostic criteria in evaluating an individual for PTSD, along with risk factors for PTSD and the impact of each criterion on the individual's functioning. The need for research on private sector EMTs was discussed, after which the relevant variables for the study was introduced with the rationale for the inclusion of each variable in the study. The proposed model for the study, demonstrating the conceptual framework for the study, was discussed next, followed by the stated purpose of the study and the research questions that guided the study. The importance of the research on private sector EMTs was proposed, after which the limitations and delimitations were presented. Closing this first chapter was a setting forth of the important and relevant terms for the study.

Chapter two discussed the research that has taken place in the field in relation to PTSD and the populations that have been studied in terms of PTSD and its outcomes. A listing of search terms that were used in identifying relevant research was presented, and a break-down of the multiple factors related to the research behind PTSD was discussed.

Chapter three began by addressing the purpose of the study, and reintroduced the research questions for the study. The research design was presented, as well as the population and sample for this study. The instrumentation that was used for this study was expressed, as was the procedure that the study utilized. How the data was obtained and what the process consisted of in terms of ensuring the appropriateness of the evaluative process statistically was discussed 
next, with a discussion of the analysis of the data. Finally, a summary of this information was presented to the reader.

Chapter four contained a presentation of the research findings/results, including statistical analyses and the outcome of each of the research questions. The final chapter, chapter five, contained a discussion of the results of the study, along with an interpretation of the findings and the implications for future study. 


\section{CHAPTER 2}

\section{LITERATURE REVIEW}

\section{Post-Traumatic Stress Disorder in Field Emergency Medical Technicians}

Posttraumatic Stress Disorder (PTSD) has a long history of research backing it, with initial research being performed on military populations, and on military veterans. It was initially believed that only military individuals could experience PTSD, but as research has continued in the field, it has become clear that anyone who has been exposed to a traumatic event or who has become aware of someone who has, can experience PTSD. The purpose of this research study was multiple. It was the goal of this researcher to: 1) identify the literature in the field associated with PTSD as it is related to private sector field Emergency Medical Service (EMS) workers in order to identify and evaluate the work that has been done in this area, 2) identify any areas of controversy related to PTSD in private sector field Emergency Medical Technicians (EMTs), and 3) provide a summary of the research to evaluate how coping style, emotional intelligence, resilience, and attachment impact the effect of the exposure to traumatic events on field EMTs. Additionally, it was the goal of this researcher to determine the presence of PTSD following such exposure, as well as to formulate questions that need to be addressed in future research.

\section{Data Sources and Searches}

The following sources were searched for research on PTSD: PsycINFO;

PsycARTICLES, EBSCO Host; Sage Publications; ScienceDirect; Science Citation Index; Social Sciences Index; PubMed; Proquest; and Proquest Dissertations and Theses. All data was searched between January 2016 and May 2018. Keywords that were utilized included Posttraumatic Stress Disorder, PTSD, Posttraumatic Stress Disorder in Emergency Medical 
Service Workers, Posttraumatic Stress Disorder in EMS Workers, PTSD in Emergency Medical Service Workers, PTSD in EMS Workers, Cognitive and Affective Empathy, Cumulative Stress, Coping, Coping and PTSD, Coping Skills, Coping Style, Emotional Intelligence, Emotional Intelligence, Attachment, Attachment and Trauma, Attachment and Coping, Attachment in EMS, Resilience, Resiliency in EMS Workers, Resiliency and EMTs, and Complex Trauma.

\section{Study Selection}

Inclusion and exclusion criteria were identified for variables related to the search for literature. In order to be eligible for inclusion in this study, individuals were required to either be working in the field as an EMT, or to have worked as a field EMT within the previous 5 years. Individuals who had never worked in the field as EMTs, or who had worked greater than five years prior to this study were excluded from inclusion. This literature review examined the diagnostic criteria for PTSD, and identified the biological correlates associated with it, including debates within the field, as related to the biology of PTSD. It also examined the research related to coping styles, emotional intelligence, and attachment. It examined risk factors for PTSD, including the epidemiology and prevalence rates for it. Finally, current treatments were discussed, as was the health risks associated with PTSD, and protective factors for PTSD.

\section{Post-Traumatic Stress Disorder}

While mental health workers are trained in a multitude of treatment areas, there remains the need for workers to become more aware of the many populations that may experience PTSD and how this plays out in the lives of those experiencing it. However, in order to be able to identify what PTSD is, one must understand the diagnostic criteria related to it. 


\section{Definition and Diagnostic Criteria}

The Diagnostic and Statistical Manual of Mental Disorders (DSM-5) classifies PTSD as a “Trauma and Stressor-Related Disorders" (APA, 2013, p. 265) which is designated for disorders that are inclusive of "disorders in which exposure to a traumatic or stressful event is listed explicitly as a diagnostic criterion" (p. 265). Criteria for diagnosis includes "witnessing or directly experiencing a traumatic event, learning about the traumatic events that happened to a close friend or family member, or repeatedly experiencing the aversive effects of a traumatic event; the presence of intrusion symptoms following the association with the traumatic event; a persistent and direct avoidance of stimuli that is associated with the traumatic event" (p. 271); “experiencing negative moods and altered mental cognitions, a change in one's arousal state and reactivity that are associated with the traumatic event; the duration of these states and symptoms for a period greater than one month; disturbance, not associated with the psychological effects of a substance or another medical condition" (p. 272), that "significantly impairs the individual or creates distress in one or more areas of functioning in their life" (pp. 271, 272). Specifiers for diagnosis may include "dissociative symptoms, a sense of derealization, and/or a sense of depersonalization, and also need to specify whether the expression of PTSD symptomatology is immediate or delayed" (p. 272). Bomyea, Risbrough, and Lang (2012) define PTSD as a "pathological response to a traumatic event such as combat, natural disasters, or physical or sexual assault" (p. 2), while Kremen, Koenen, Afari, and Lyons (2012) others define it as "an outcome that is defined by one's response to an environmental event" (p. 2). A third class of researchers use DSM criteria to define PTSD referring to it as "an anxiety disorder that manifests in response to a traumatic event that involves an actual or perceived threat of death or injury" (Mišić, Dunkley, Sedge, Da Costa, Fatima, Berman, Doesburg, McIntosh, Grodecki, Jetly, Pang, 
\& Taylor, 2016, p. 1; Acheson, Gresack, \& Risbrough, 2012; APA, 2000 as cited in Mišić et al, 2016).

While a great deal of research has focused on the multitude of variables that may help to explain the causes of PTSD, there are currently no studies that have examined the relationship between coping skills, attachment, resilience, and emotional intelligence on the development and expression of PTSD in private sector EMTs. Some of the areas that research has focused on include the neurobiology of PTSD, general factors that may possibly predispose individuals to develop PTSD, early childhood factors and experiences that have been shown to contribute to the development of PTSD, such as abuse and neglect, biological factors, such as heredity and DNA, and factors that could affect the symptom expression of each of the four clusters (reexperiencing/intrusion symptoms, avoidance, negative cognitions and mood, and arousal symptoms).

\section{Impact of Post-Traumatic Stress Disorder}

The effect of PTSD has been well documented, with PTSD being the most commonly diagnosed (Green \& Kaltman, 2003) disorder resulting from exposure to trauma (Matusko, Kemp, Paterson, \& Bryant, 2009). It is estimated that about $25 \%$ of people who are exposed to a traumatic event will develop PTSD (Green \& Kaltman, 2003). Some of the outcomes of trauma-related stressors include greater anger expression (Horn, Miller-Graff, Galano, \& Graham-Bermann, 2017; Germain, Kangas, Taylor, \& Forbes, 2016), changes in the neuroendocrine system (Le Dorze, \& Gisquet-Verrier, 2016; Kremen, Koenen, Afari, \& Lyons, 2012; Kanitz, Puppe, Tuchscherer, Heberer, Viergutz, \& Tuchscherer, 2009), changes in the ability to think and process information (Kremen et al, 2012), changes in sleep and circadian functioning (Silver, \& LeSauter, 2008), marital and other relational stressors (Vogel, Cohen, 
Habib, \& Massey, 2004), emotional distress (Bennett, Williams, Page, Hood, Woollard, \& Vetter, 2005), and suicidality (Ali, 2016; Willing, 2011 as cited in Ali, 2016) to name a few.

\section{Biological Factors in Post-Traumatic Stress Disorder}

The study of biological factors that have been shown to be possible precursors to the development of PTSD, and that may have a relation to PTSD include postmortem studies on brain structures, identical twin studies, and imaging techniques on live individuals who are experiencing PTSD.

\section{Twin studies}

Twin studies have been the primary means of research on biological factors that may be related to PTSD. Kremen, Koenen, Afari, and Lyons (2012) state that these studies have been made in "an effort to resolve the 'chicken-egg' dilemma inherent in standard case-control designs" (p. 2), as well as to enable the identification of abnormalities that may possibly indicate environmental pre-existing vulnerability factors (Kremen et al, 2012). The manner that these pre-existing vulnerabilities would be identified would be in the expression of factors that are related to the development of PTSD as identified as existing in both twins, whereas, abnormalities that are expressed in just one of the twins suggests that these are environmental factors, due to these not being evidenced in both twins (Kremen et al, 2012). Environmental factors would include things such as exposure to a traumatic event (Kremen et al, 2012).

Researchers found what they defined as "neurological soft signs" (Kremen et al, 2012, p. 2), in a meta-analytic study performed by Kremen, Koenen, Afari, and Lyons (2012), which were identified as lower overall general intellectual abilities, poorer cognitive performance that were seen in the areas of the processing of contextual cues, attention, declarative memory, and executive functioning (Kremen et al, 2012). They also found physical changes in several brain 
structures and areas within the brain. These included smaller hippocampal volume and reduced gray matter density in those with PTSD and larger cavum septum pellucidum (Kremen et al, 2012). Each of these were found in the twin study results, in which the twin with PTSD evidencing these changes in brain structure, while the other twin did not. However, as opposed to this, some researchers have found no differences in twins in which one of the twins was diagnosed with PTSD and the other was not. Some of these studies included things such as “psychophysiological responding, higher resting anterior cingulate metabolism, event-related potential abnormalities associated with attentional processes, recall intrusions, and possibly some types of chronic pain” (Kremen et al, 2012, p. 2).

\section{Neuroimaging techniques}

A plethora of neuroimaging techniques are available, and have been used in the examination and identification of PTSD. Some of these include the use of have included the use of fluorodeoxyglucose positron emission tomography (FDGPET), magnetic resonance imaging (MRI), functional magnetic resonance imaging (fMRI), computed tomography (CT), blood oxygen level dependent fMRI (BOLD fMRI), magnetic resonance spectroscopy (MRS), diffusion tensor imaging (DTI), susceptibility-weighted imaging (SWI), and perfusion weighted MRI (PW-MRI). Detailed images of the brain and its structures are able to be seen with MRI's, while positron emission tomography (PET) uses radioisotopes to produce 3-dimentional colored images that help researchers to visualize where brain activity is taking place while the individual performs specific tasks (Kumar, 2008). FDGPET, a form of PET, uses the radioisotope fluorodeoxyglucose as this is known to be much more sensitive than conventional imaging techniques (Kumar, 2008). In this procedure fluorodeoxyglucose is injected into the individual's vein, multiple samples of arterial blood are drawn and sampled to measure and to visualize 
which structures are activity in the brain during cognitive tasks and processes, and is being used as a means of imaging in the assessment of psychiatric disorders (Newberg, A., Alavi, A., \& Reivich, M., 2002; Wakita, Imahori, Ido, Fujii, Horii, Shimizu, Nakajima, Nakamura, \& Kanatsuna, 2000 ).

Brain structure is also examined through the use of CT and X-rays (Watson \& Breedlove, 2016), and fMRI's are useful in "detecting changes in blood flow" (Watson \& Breedlove, 2016, p. 44) as a means of identifying "regions of the brain that are particularly active during a given task" (Watson \& Breedlove, 2016, p.44). BOLD fMRI's work by phlebotomists taking indirect measures of blood flow that signal changes in venules (smaller veins) which measure the differences between oxygenated and deoxygenated blood as a way of evaluating brain activity as related to specified cognitive tasks (Brenner, 2011, p. 321 as cited in Van Boven et al., 2009). The way DTI is used is an indirect measure of the diffusion of water molecules to that identify both disease and "structural connectivity" between specific brain structures and regions (Brenner, 2011, p. 321 as cited in Van Boven et al., 2009). MRS is used to "evaluate changes in brain metabolites related to myelination and neuronal density (Brenner, 2011, p. 321 as cited in Van Boven et al., 2009), and PW-MRI is a "direct measure of blood flow, and allows quantification of blood perfusion similar to fMRI's" (Brenner, 2011, p. 321 as cited in Van Boven et al., 2009). Finally, SWI measures "MRI sequences that are especially sensitive to changes in magnetic susceptibility" (Brenner, 2011, p. 321 as cited in Van Boven et al., 2009), and provides the researcher with an improved means of the "imaging of blood vessels" (Brenner, 2011, p. 321 as cited in Van Boven et al., 2009). 


\section{Affected brain structures}

Identified brain structures that have been shown to have an association with the expression and exacerbation of PTSD symptomatology include the hippocampus, amygdala, insula, thalamus, HPA (hypothalamic-pituitary-adrenal axis), posterior parietal cortex, temporal lobes, cingulate cortex, and the prefrontal cortex (Tyrka, Burgers, Philip, Price, \& Carpenter, 2013; Antunes-Alves \& Comeau, 2014; Marin, Camprodon, Dougherty, \& Milad, 2014; Brenner, 2011; Lanius, Bluhm, \& Frewen, 2011).

\section{Hippocampus}

The hippocampus is associated with "explicit and declarative memory, working memory, and episodic/autobiographical memory" (Brenner, 2011, 315), and has been shown to be "critical for encoding memories in which a complex configuration of multiple cues is associated with the aversive event, but is not required for associations with discrete cues," (Acheson, Gresack, \& Risbrough, 2012, p. 2). Further, researchers have proposed that "poor hippocampal function may bias the organism towards forming multiple simple cue associations during trauma, thus increasing the chances of fear responses in multiple environments or contexts in which these cues may be present" (Acheson, Gresack, \& Risbrough, 2012, p. 2). BOLD fMRI studies have found greater activation in the hippocampus of individuals who had encoded negative imagery (Brohawn, Offringa, Pfaff, Hughes \& Shin, 2010 as cited in Acheson, Gresack, \& Risbrough, 2012, p. 11), and that "reduced hippocampal activation was associated with increased arousal symptoms. (Acheson, Gresack, \& Risbrough, 2012, p. 11; Brenner, 2011, p. 322). Both reduced hippocampal function and decreased hippocampal volume have been found in individuals with PTSD (Antunes-Alves \& Comeau, 2014). 


\section{Amygdala}

"The amygdala is responsible for the generation and maintenance of emotional responses" (Brenner, 2011, p. 315), and for responding to impending danger (Antunes-Alves \& Comeau, 2014). Individuals with PTSD have been shown to demonstrate much more exaggerated fear and startle responses (Brenner, 2011), fear conditioning, emotional and social processing, and modulation of memory consolidation” (Lanius, Bluhm, \& Frewen, 2011, p. 333).

\section{Prefrontal cortex}

Self-reflection, self-awareness, and the monitoring and modulation of emotions is found in the prefrontal cortex (PFC) as maintained through a strong connection with the limbic system (Lanius, Bluhm, \& Frewen, 2011, p. 333). It is the prefrontal cortex that has traditionally been thought of as the area responsible for "complex thought and operations, decision-making, attention, impulsivity, personality, and emotionality" (Antunes-Alves \& Comeau, 2014, p. 10), for the manipulation of memories and emotions, for the extinguishing of conditioned fear, and for inhibiting the action of the amygdala. This area is instrumental in "inhibiting the amygdala and extinguishing fear responses” (Antunes-Alves \& Comeau, 2014, p. 10; Brenner, 2011, p. 315). Findings among individuals with PTSD show decreased activity in the PFC (AntunesAlves \& Comeau, 2014), and deficient prefrontal functioning (Brenner, 2011, p. 322) as exhibited by decreased and greater hypoactivity, which has been associated with more severe PTSD symptoms (Antunes-Alves \& Comeau, 2014).

\section{Insula}

The insula is responsible for "interoceptive awareness of bodily states, bodily selfawareness, regulation of sympathetic and parasympathetic systems, and sensation of pain" (Lanius, Bluhm, \& Frewen, 2011, p. 333), and focus on the insula has demonstrated the 
hyperactivation of the insula as shown in anxious individuals (Brenner, 2011, p. 322). It is responsible for "core affect, consciousness of subjective feelings, for developing and updating motivational states, cognitive control, affective processing, pain, conveyance of homeostatic information, and autobiographical memory" (Brenner, 2011, p. 315).

\section{Hypothalamic-pituitary-adrenal axis}

The hypothalamic-pituitary-adrenal (HPA) axis is the structure that allows the body to respond to effectively to the experience of stress. In this system, corticotrophin releasing factor $(\mathrm{CRF})$ is released by the paraventricular nucleus of the hypothalamus, which then activates CRF receptors at the pituitary. The pituitary then releases adrenocorticotropic hormone into the blood stream, inducing the adrenal glands to activate the peripheral release of cortical glucocorticoids (e.g., cortisol). Glucocorticoids then act to mobilize physiological changes such as release of glucose and lipid stores, and they increase immune response (Breedlove, Rosenzweig, \& Watson, 2007). Glucocorticoids have also been found to be transported to the central nervous system, at which point they act to modify neural plasticity and behavior, and to shut off CRF release (negative feedback). Although this response is protective and useful for mobilizing resources within the body to cope with threat, and which typically terminates the return of the body to a homeostatic state, chronic or particularly severe stressors, such as trauma, may lead to disruptions in this system. This may result in the body being exposed to the prolonged release of stress hormones and cortisol (Bomyea, Risbrough, \& Lang, 2012, p. 7). In addition to this, the HPA also helps to regulate the body's physiological responses to stress, which makes this area particularly vulnerable to PTSD (Bomyea, Risbrough, \& Lang, 2012, p. 8). Finally, in relation to glucocortotropins, PTSD patients have been found to have elevated levels of corticotropinreleasing hormone $(\mathrm{CRH})$ in their cerebrospinal fluid of (Schmidt, Kaltwasser, \& Wotjak, 2013, 
p. 46). Individuals with PTSD have been shown to demonstrate a decrease in activity levels of the HPA, which, working in conjunction with the amygdala, contributes to the maintenance of PTSD symptoms (Antunes-Alves \& Comeau, 2014).

\section{Cingulate cortex}

The posterior cingulate cortex has been shown to be responsible for "integrating cognitive and emotional aspects of experience, assessing the salience of emotional and motivational information, regulating the autonomic nervous system (heart rate, blood pressure) and for changes to emotional stimuli, pain processing, attention to error processing, sustained attention and cognitive control, self-referential processing, and episodic memory retrieval" (Lanius, Bluhm, \& Frewen, 2011, p. 333). It has also been found to be instrumental in processing emotional and cognitive interactions, including the interfering of emotional stimuli, in error detection, and for conflict monitoring, attention, and pain (Brenner, 2011, p. 315). 


\section{Table 1}

Brain Regions and Neurochemical Dysfunction Associated with PTSD

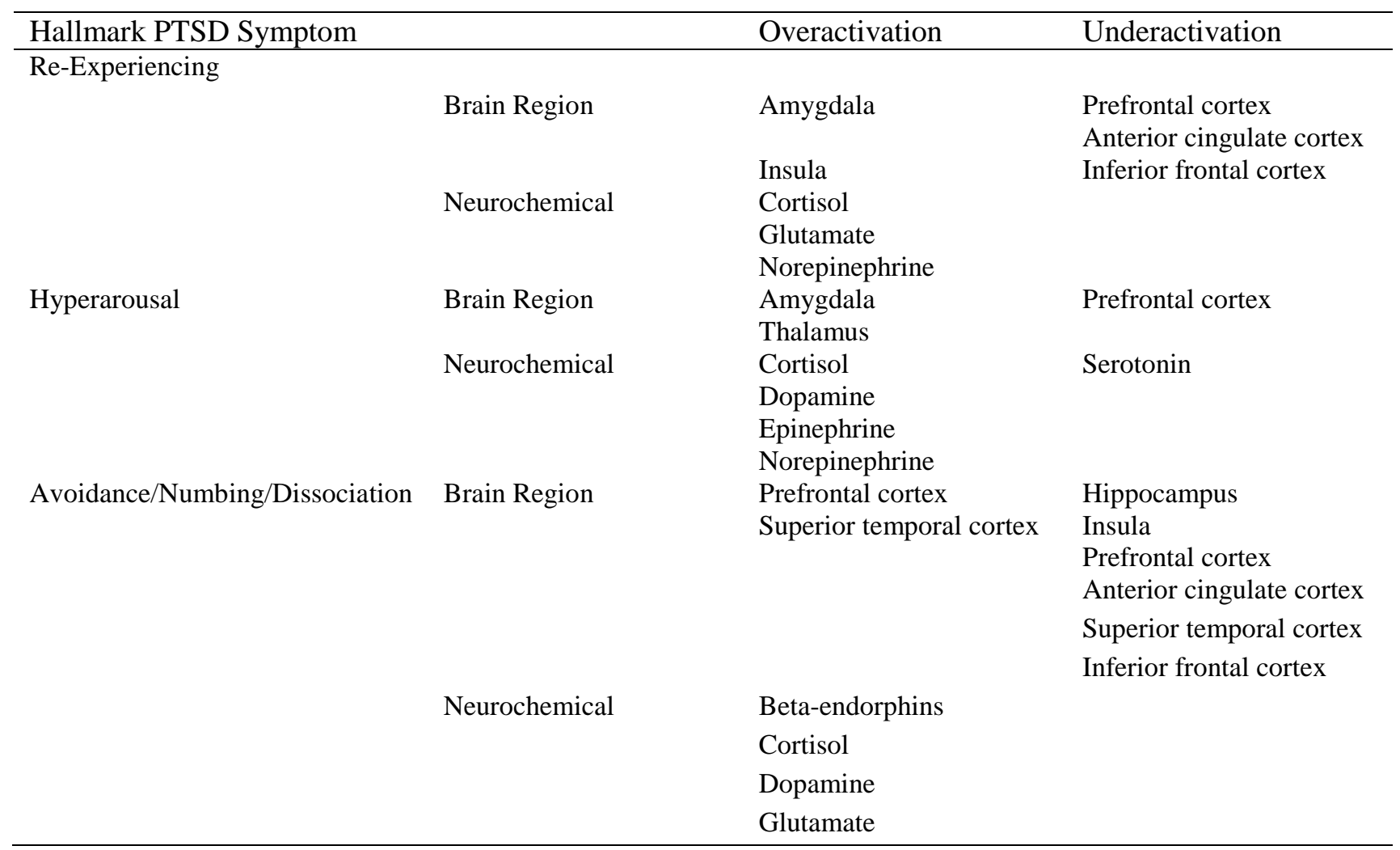

Note. Hopper, Frewen, van der Kolk, \& Lanius, 2007, p. 314.

\section{Structures and symptoms}

Table 1, above, shows the hallmark PTSD symptoms, which brain structure is associated with the overacted symptoms, and which brain structures are associated with under-action of functioning. This Table is taken directly from page 314 of Brenner's article, Neuropsychological and neuroimaging findings in traumatic brain injury and post-traumatic stress disorder, as is replicated from the research by Hopper, Frewen, van der Kolk, and Lanius (2007).

\section{Childhood Factors}

In addition to the examination of biological factors as implicated in providing evidence from identical twin studies of factors that may provide researchers in identifying variables that 
may lead to the development of PTSD, it is also necessary to examine childhood factors that may also indicate the trajectory of individuals developing PTSD.

\section{Childhood abuse}

Tyrka, Burgers, Philip, Price, and Carpenter (2013) highlight the seriousness of childhood abuse in implicating childhood maltreatment as an "environmental exposure that acts much like a toxin, with the potential to influence several neurobiological systems implicated in the pathophysiology of these disorders" (p. 2). Neuroendocrine changes, genetic changes, and neurobiological changes are just a few of the effects that result from childhood maltreatment and abuse (Tyrka, Burgers, Philip, Price, \& Carpenter, 2013). Tyrka, Burgers, Philip, Price, and Carpenter (2013) report that early life stressors as being associated with changes in both the nervous system, as well as in the hypothalamic-pituitary-adrenal axis (HPA). Tyrka, Burgers, Philip, Price, and Carpenter (2013) describe this process as releasing the following hormones corticotropin-releasing hormone $(\mathrm{CRH})$ and arginine vasopressin (AVP) - as a result of child abuse and neglect, which then change sympathetic arousal in the abused individual. They report that "these two hormones act in conjunction with one another, producing the release of adrenocorticotropic hormone (ACTH), which then stimulates the release of glucocorticoids, of which cortisol is the most potent and abundant" (Tyrka et al, 2013, p. 3). The sympathetic nervous system then releases catecholamines, which are instrumental in the functioning of individuals in response to stress, allowing and assisting in the appropriate and adaptive response to stressors in one's life (Tyrka et al, 2013). Individuals who live under the constant threat of fear of stress, which is the most prominent condition under which abused children live, are physiologically able to benefit from "regulated homeostasis, with negative feedback from cortisol acting at hypothalamic and pituitary glucocorticoid receptors to terminate the acute stress 
response" (Tyrka et al, 2013, p. 3). Smaller volumes in the hippocampus, prefrontal cortex, and anterior cingulate have been shown to have a link to Cortisol, which also results in decreased connective functionality between the amygdala and the prefrontal cortex (Carrion, Weems, Richert, Hoffman, \& Reiss, 2010 as cited in Tyrka et al, 2013; Veer, Oei, Spinhoven, Van Buchem, Elizinga, \& Rombouts, 2012 as cited in Tyrka et al, 2013; Treadway, Grant, Ding, Hollon, Gore, \& Shelton, 2009 as cited in Tyrka et al, 2013).

"When stressors are excessive or prolonged, attempts at maintaining homeostasis can lead to alterations of basal and provoked HPA axis activity and associated functioning of autonomic, metabolic, and immune systems" (Tyrka et al, 2013, p. 3). Prolonged stress also results in altered neuroendocrine function, with this being seen in adults who present with mental health disorders such as PTSD (Tyrka et al, 2013). In addition to this, epigenetic changes have been found in the gene for the degradation of catecholamines, catechol-O-methyltransferase (COMT) (Tyrka et al, 2013), which means that catecholamines will remain in the neuronal gap to be used by the nervous system, resulting in increases in dopamine and norepinephrine (Kobayaski, 2001). The consolidation of long-term memory relies on Norepinephrine, which may also possibly control neuronal activity in both the amygdala and the cerebral cortex, while dopamine is instrumental in motor control and emotional learning (Kobayaski, 2001). The damage of these two hormones and systems result in fear that is learned in traumatic situations becoming conditioned as a learning experience, and may also result in the hardwiring of fear as the primary response process (Kobayaski, 2001; Schmidt, Kaltwasser, \& Wotjak, 2013).

\section{Risk Factors for PTSD}

According to a meta-analysis performed by Acheson, Gresack, and Risbrough (2012), risk factors for developing PTSD include genetic and epigenetic factors, such as early life 
stressors, that contribute to an increase in the expression of stress responses in adulthood (McGowan et al., 2009 as cited in Acheson, Gresack, \& Risbrough, 2012). Included in this are childhood abuse (Tyrka et al, 2013) and being female (Malta, 2012; Schmidt, Kaltwasser, \& Wotjak, 2013; Tyrka et al, 2013), previous exposure to trauma (Tyrka et al, 2013, and having a family history of psychopathology (Brewin, Dalgleish, \& Joseph, 1996 as cited in Bomyea, Risbrough, \& Lang, 2012; Ozer, Best, Lipsey, \& Weiss, 2003 as cited in Bomyea, Risbrough, \& Lang, 2012; Tyrka et al, 2013).

\section{Similarities and Differences in Studies}

Research on identical twins appears to reveal the same information from research study to research study, although this was not always the case due to earlier twin studies concluding that that brain abnormalities were the result of, or squelae, of earlier injuries (Kremen et al, 2012, p. 6). However, most researchers now believe that much of the resulting damage as shown in specific brain structures, such as the presence of positive symptomatology, and changes in brain functioning, as being due to combat exposure, and that these have actually served as protective factors for those exposed to traumatic situations.

Because most of the research on PTSD has focused on military veterans who present with symptoms of PTSD, and has focused much less on populations of individuals who have been exposed to traumatic events and are presenting with the same symptoms of PTSD, most of the literature references combat exposure as the primary means of exposure to a traumatic event. This leaves the field open to the necessity of additional study with other populations that have been exposed to and have experienced traumatic events. This has become more important as professionals in the field have begun to recognize that PTSD is demonstrated in individuals who are exposed to persistent life stressors (abused children, individuals living in intimate-partner 
relationships), those who work in environments where there are either consistent or one-time incidents of severe traumatic events (EMTs, first responders, fire fighters, police, etc.), those who survive traumatic events (tsunami's, floods, tornado's, hurricane's, earthquakes, etc.), and those who may experience PTSD vicariously through others, or on a secondary basis.

Further, there is a need for continued studies related to PTSD, including twin studies, since most identical twin control studies related to PTSD have been small, and they have focused primarily on male veterans (Kremen, Koenen, Afari, \& Lyons, 2012). These researchers propose that there is a need for replication of the studies that are presently in place, and that these studies must now be extended to include both the similarities and the differences of women and younger individuals.

\section{Debates in the Literature}

Debates in the literature include some disagreement related to the findings of research studies, such as those focusing on the deficits in verbal declarative memory in patients with PTSD. According to Acheson, Gresack, and Risbrough (2012), who cite the following parenthetical studies: (Gilbertson et al., 2006; Buckley et al., 2000; Elzinga \& Bremner, 2002; Brewin, 2001; Golier \& Yehuda, 1998; Bremner et al., 1993; Bermner et al., 1995; Gilbertson et al., 2001; Jenkins et al, 1998; Moradi et al., 1999; Roca \& Freeman, 2001; Uddo et al., 1993; Vasterling et al., 1998; Vasterling et al., 2002; Yehuda et al., 1995; Barrett et al., 1996; Gil et al., 1990; Sachinvala et al., 2000; Golier et al, 1997) (p. 9), report that some researchers have found present deficits, while other studies have found none (Stein et al., 1999; Zalewski et al., 1994) (p. 9). The authors cite Brewin and colleagues (2007 as cited in Acheson et al, 2012) as having reviewed 27 studies related to visual and verbal memory differences between individuals, with PTSD and control individuals, with PTSD showing to be a neutral response (p. 9). They report 
that the results "consistently supported a small to moderate effect for poorer performance on verbal as opposed to visual memory in PTSD subjects" (Acheson, Gresack, \& Risbrough, 2012, p. 9), with this pattern of results "suggesting that declarative memory is an important link between hippocampal abnormalities and PTSD symptomatology" (Acheson, Gresack, \& Risbrough, 2012, p. 9). However, they report that Woodward et al. (2009 as cited in (Acheson, Gresack, \& Risbrough, 2012, p. 9), "while observing verbal declarative memory deficits in PTSD patients, failed to find any correlation between these deficits and hippocampal volume" (Acheson, Gresack, \& Risbrough, 2012, p. 9), despite the fact that the PTSD patients in their study sample showing "significant volume reductions relative to controls in the hippocampus, parahippocampus, and total cerebral cortex" (Acheson, Gresack, \& Risbrough, 2012, p. 9).

In addition to these studies, Acheson et al (2012) also propose that researchers use caution when interpreting fMRI studies related to the BOLD results with task performance and stimulus processing due to the difficulty in interpreting BOLD results. They propose that "increases in $\mathrm{rCBF} / \mathrm{BOLD}$ response can be interpreted as a lack of efficiency and that this region is "working harder" than the same region in the comparison group or simply that it is more engaged by the task" (Acheson, Gresack, \& Risbrough, 2012, p. 9), and that "low levels can be interpreted that the region is either not functionally engaged in the task or the opposite, that it is engaged and working with more "efficiency" (Acheson, Gresack, \& Risbrough, 2012, p. 9). Acheson et al (2012) further report inconsistent results in "directional changes compared to controls" (Acheson, Gresack, \& Risbrough, 2012, p. 9), and state that this may have been due to the tasks engaging "different hippocampal processes (response to trauma cues, spatial navigation learning, declarative memory recall), and thus likely engaging different hippocampal neural circuits (e.g. hippocampus-cortex vs. hippocampus-amygdala)" (Acheson, Gresack, \& 
Risbrough, 2012, p. 9). They conclude that, nevertheless, the findings of BOLD studies on PTSD suggest abnormalities in the hippocampus (Acheson, Gresack, \& Risbrough, 2012).

Outside of these reported studies, PTSD has been associated with decreased basal or stress-induced cortisol concentrations in several studies, but with increased ACTH and/or cortisol concentrations in others (Tyrka et al, 2013). Tyrka et al (2013) assert that a great problem with this is that glucocorticoid receptors have been found in many structures within the limbic system, including the hippocampus, the prefrontal cortex, the hypothalamus, and the amygdala, with one of the results of stress on these glucocorticoid receptors being a "remodeling and inhibition of cell proliferation in the prefrontal cortex" (Tyrka et al, 2013, p. 3), but that this results in "neuronal proliferation and dendritic growth" (Tyrka et al, 2013, p. 3). The outcome of this is that this process greatly increases the activation of the amygdala and the hippocampus, and that it results in the reduction of the use of the prefrontal cortex. This, then, results in increased memory for traumatic events, increased fear and anxiety, and the reduced ability to make effective and adequate choices. The stress activation is noted to be a possible factor in the development of PTSD later in life (Tyrka et al, 2013).

\section{Psychological Factors in Post-Traumatic Stress Disorder}

\section{Coping Style}

\section{History and foundation}

Coping style has been well researched in multiple settings and with multiple populations. Perhaps the first and well-known researchers in the field were Lazarus and Folkman (Folkman \& Lazarus, 1980; Folkman \& Lazarus, 1985), with Lazarus (1966) serving as the pioneer, offering a model of stress and coping which served as the foundation for all subsequent and current research on coping. Lazarus (1966) proposed a three-part process to coping with stress, which 
included primary and secondary appraisals as the initial steps in the process that then leads to the actual coping process. The perception of threat was the first step, which was identified as the primary appraisal of the stressor (Carver, Scheier, \& Weintraub, 1989). The second step, secondary appraisal, is the step in which one identifies a response to the perceived threat (Carver et al., 1989). The final step is the coping response, which Carver, Scheier, and Weintraub (1989) assert is the execution of the response to the threat.

In order to study the coping aspect of the response to stress, Folkman and Lazarus (1980; 1985) formulated the Ways of Coping measure. Two primary forms of coping were identified, which were as follows: those proposed to be those focused the actual stressor and its source, named problem-focused coping; and those focused on the emotions that arise as a response to the stressor and managing those emotional reactions, named emotion-focused coping (Folkman \& Lazarus, 1980). However, as research continued in the field, it was determined that these two coping responses were too simple, and that there were multiple other factors that came to be identified through the use of the Ways of Coping scale (Aldwin, Folkman, Schaefer, Coyne, \& Lazarus, 1980; Coyne, Aldwin, \& Lazarus, 1981; Parkes, 1984; Folkman \& Lazarus, 1985; Folkman, Lazarus, Dunkel-Schetter, DeLongis, \& Green, 1986; Scheier, Weintraub, \& Carver, 1986; Aldwin \& Revenson, 1987; Carver, Scheier, \& Weintraub, 1989). In 1981, Billings and Moos examined coping in children, and proposed coping as consisting of either an active or a passive style, or an "approach" versus and "avoidant" focus (Billings, \& Moos, 1981; Ayers, Sandler, West, \& Roosa, 1996). Passive coping was asserted to be indirect, and were utilized to avoid having to deal with the stressor or its outcome, while active coping is a direct approach to engage with the stressor, so to speak, and to master it. Ebata and Moos (1991) state the coping styles to be as follows: Active/approach coping reflects "active cognitive and behavioral efforts 
to define and understand the situation and to resolve a stressor by seeking guidance and engaging in problem solving activities" (Ebata \& Moos, 1991, p. 36); and passive/avoidant responses as reflecting "cognitive or behavioral attempts to avoid thinking about a stressor or its implications, to accept or resign oneself to an existing situation, to seek alternative rewards, or to try to manage tension by expressing it openly" (Ebata \& Moos, 1991, p. 36). However, as with Lazarus and Folkman's two-factor model (Folkman \& Lazarus, 1980) this too was much too simplistic to fit the data that was gathered in relation to coping skills and styles.

While each of these research studies dates back anywhere from 30 to 50 years, without this foundation, it would be impossible to understand or to be able to discuss the more current research in the field as related to coping. Continuing on, then, we can see that researchers in the field began to identify additional factors relevant to coping by moving from the two-factor model as proposed by Lazarus and Folkman $(1980 ; 1984)$ consisting of problem-focused versus emotion-focused coping, and the active versus passive coping model as proposed by Billings and Moos (1981), both of which were discussed above, to a four-factor model.

Ayers, Sandler, West, and Roosa (1996) assert that coping response is a multidimensional structure, of which previous coping theories and models were unable to identify, for both children and for adults. Accordingly, they proposed that emotional coping responses hold variations, that "have quite different implications for adaptation" (Ayers, Sandler, West, \& Roosa, 1996, p. 927), and which "reflect such dimensions as expression of feelings, positive reinterpretation of the event, seeking social support, avoidance, or denial" (Ayers et al., 1996, p. 927). Confirmatory factor analysis was used to evaluate in greater depth their theoretical formulation of coping, after which a four-factor model was identified. 
The results of their research found that avoidance strategies, distraction strategies, active coping strategies, and support-seeking strategies were a better fit for the data than the previous two-factor models (Ayers et al., 1996, p. 940). Additionally, Ayers and colleagues (1996) affirm that the outcome of their research was confirmed through "moderate to high correlations" between their model and "similar subscales of dispositional and situational measures of coping" (p. 951). Their research also demonstrated support for this four-factor structure regardless of age or gender (Ayers et al., 1996). These findings support the use of research studies on both children and adults as being relevant in informing the current evaluation on field EMTs. This is supported by the research findings of Dalgleish, Meiser-Stedman, and Smith (2005), who assert that similarities in the manner in which children and adults process traumatic events and resulting PTSD outcomes indicate the applicability of current models to both adults and children (Stallard and Smith (2007).

For this study, we used the description of coping as proposed by Carver and Scheier (1994) is stated to be the manner in which people react to and cope with stressors and difficulties in their lives, and the tools that they utilize in response or reaction to those stressors and while under stress (Carver \& Scheier, 1994). His measure, the Brief COPE, identified four coping subscales: Use of Emotional Social Support; Use of Problem Solving; Avoidance Coping; and Use of Positive Thinking.

\section{Previous decade}

Following the four-factor model identified by Ayers and colleagues (1996), researchers began to implement these factors in their exploration of how coping style impacted the presence of stressful situations in an individual's life. Bryant, Marosszeky, Crooks, Baguley, and Gurka (2000) examined coping style in relation to PTSD in patients with severe traumatic brain injury 
(TBI), and found that avoidant coping style predicted PTSD severity. Avoidant coping was also found to predict PTSD symptom severity over time in a longitudinal study on Scandinavian individuals by Jamessen, Eid, Laberg, and Thayer (2002).

Gil (2005) examined PTSD in Israeli students who had been exposed to a terrorist attack, and found four factors to predict PTSD. As part of their examination all factors were broken down into their state and trait subcomponents. Both state and trait components of avoidance coping predicted PTSD, as did trait emotion-focused coping. Understanding traits to be components that are more long-lasting in an individual's life, and state's being more specific to situational factors, these findings help to provide greater understanding in the field to factors that can be targeted in the treatment of PTSD. Gil's (2005) findings indicate that avoidant coping in any form, serves to be detrimental to those exposed to traumatic events, while emotion-focused coping that is indicative of an individual's commonly utilized coping style despite their situation proved to be the aspect of emotion-focused coping that was detrimental to dealing effectively with stressors in the individual's life. However, although not a trait factor, problem-focused coping proved to be an asset when dealing with traumatic events (Gil, 2005). Finally, avoidant emotional coping was found to be the one significant factor following "path analysis in controlling for time since loss and trauma frequency in predicting PTSD severity" (Schneider, Elhai, \& Gray, 2007, p. 344) in college students who had reported a previous traumatic loss.

\section{Current research}

As the research on coping has continued on, the evidence that avoidant coping has a direct correlation with PTSD symptom severity has remained robust. A 2012 study examined refugees that had settled in the Netherlands following war, persecution, and violence, with the study outcomes again demonstrating a link between PTSD severity and avoidant coping (Huijts, 
Kleijn, can Emmerik, Noordhof, \& Smith, 2012). These same results were found in a study on Israeli veterans who had been diagnosed with PTSD (Galor, \& Hentschel, 2012). The final study published in 2012 was performed on veterans who had been exposed to traumatic situations, and who were receiving mental health care services through the Veterans Administration (VA).

While this study identified emotional avoidance to be associated with PTSD symptom severity, the researchers also found that low levels of emotional expression was also associated with PTSD symptom severity (Hassija, Luterek, Naragon-Gainey, Moore, \& Simpson, 2012). Avoidant coping was the only resulting factor that demonstrated a significant association with changes in PTSD symptom severity over a 1-year period among veterans who had served in combat zones (Lee, Possemato, \& Ouimette, 2017). Finally, when examining the ability to adjust following a traumatic event, Slanbekova, Chung, Abildina, Sabirova, Kapbasova, and Karipbaev (2017) identified emotion-focused coping and the inability to manage one's emotions to be correlated with difficulties in adjustment, whereas the use of problem-focused coping and being able to manage one's emotions were correlated with greater symptom severity in couples following a divorce.

\section{Emotional Intelligence}

Emotional intelligence has been defined as the ability for one to understand their own emotions and the emotions of others, having the ability to discriminate between emotions and feelings, and having the ability to monitor one's own emotions (Brackett, \& Geher, 2006). Others have conceptualized emotional intelligence as encompassing the emotional regulation process, which utilizes the use of cognitive, emotional, and behavioral strategies in order to regulate that process (Benoit, Bouthillier, Moss, Rousseau, \& Brunet, 2010). Still others identify emotional intelligence as including the perception and expression of emotion, the ability to 
understand emotion, being able to utilize one's own emotion to assist in facilitating the thinking process, and being able to manage both one's own emotion and others' emotions (Mayer \& Salovey, 1997).

There are still others who view emotional intelligence as including not only the awareness of one's own and others' emotions, the ability to discriminate and regulate one's and others' emotions, and the ability to use emotions to facilitate thought (Matthews, Zeidner, \& Roberts, 2002), but also the ability to remain motivated and persist in spite of frustration, to delay gratification by controlling one's impulses, to appraise others' emotions accurately, to hold out hope and be able to enhance one's own life through the use of effective emotion regulation, and to be able to keep from allowing one's emotions from "swamping the ability to think" (Wagner \& Martin, 2012, p. 59). For this study, the definition of emotional intelligence as proposed by Mayer and Salovey (1997), which proposes that emotional intelligence is comprised of the ability to understand emotion, ability to utilize one's own emotion to assist in facilitating the thinking process, and the ability to manage both one's own emotion and others' emotions, including the ability to perceive and express emotion.

Emotional intelligence has been studied in adolescent refugees from the Middle East (Ghazali, 2014), young children experiencing mental health issues and trauma, including those expressing suicidal ideation (Bodzy, Barreto, Swenson, Liguori, \& Costea, 2016), youth exposed to community violence (Stokes \& Jackson, 2014), prostate and bladder cancer patients (Smith, Turner, Pati, Petrides, Sevdalis, \& Green, 2012), those diagnosed with borderline personality disorder (Gaher, Hofman, Simons, \& Hunsaker, 2013), learning disabled individuals (Chantrell, 2009), individuals with HIV/AIDS (O’Cleirigh, Ironson, Fletcher, \& Schneiderman, 2008), divorced couples (Slanbekova, Chung, Abildina, Sabirova, Kapbasova, \& Karipbaev, 2017), and 
adults with previous traumatic experiences (Benoit, Bouthillier, Moss, Rousseau, \& Brunet, 2010).

In each of the above listed research studies, it was proposed that individuals with greater levels of emotional intelligence would demonstrate lower levels of PTSD symptom severity. This was found to be the case in each of the studies, without exception. Several studies identified emotional intelligence as a mediating factor between trauma exposure and PTSD symptom severity (Gaher, Hofman, Simons, \& Hunsaker, 2013; Benoit, Bouthillier, Moss, Rousseau, \& Brunet, 2010; O'Cleirigh, Ironson, Fletcher, \& Schneiderman, 2008), and one study identifying emotional intelligence as a moderating factor between emotional expression and posttraumatic growth (Linley, Felus, Gillett, \& Joseph, 2011). Linley et al. (2011) reported that emotional intelligence was not a significant factor for women in the expression of emotions and posttraumatic growth, but that for men, those with lower emotional intelligence emotional expression was demonstrated to be a detriment to posttraumatic growth.

It would appear, then, that in men, the difference between PTSD symptom severity versus posttraumatic growth may be determined by their level of emotional intelligence, and their ability to understand and use emotion. This ability to understand and the ability to use emotion were found to be the two most significant factors in PTSD symptom expression and emotional intelligence in Ghazali's (2014) study. This would be an important factor, since the ability to understand and use emotion could quite possibly impact the outcome of exposure to traumatic events, a common and expected aspect of field work for EMTs, and the development of problematic trauma response and symptom expression.

However, very little research has been performed on the relationship between emotional intelligence and traumatic stress in EMTs, with one research article resulting from a literature 
search on emotional intelligence and emergency medical services personnel. Wagner and Martin (2012) examined emotional intelligence and proactive coping on mental health in firefighters, with their results indicating a negative relationship between emotional intelligence and traumatic stress symptomatology. While this study came the closest to examining emotional intelligence and PTSD in field EMS workers, the study looked solely at firefighters, rather than EMTs. The lack of research on field EMTs remains an unresearched and important population due to the fact that these individuals are the first line responders in traumatic incidents, both in the home as well as on the road.

Another important consideration would be the utilization of previous research on emotional intelligence between men and women, such as demonstrated in the Liley (Linley, Felus, Gillett, \& Joseph, 2011) study mentioned above. Other studies supporting these differences include the finding that women report greater levels of posttraumatic growth (Linley \& Joseph, 2004), emotional expression (Stanton, Kirk, Cameron, \& Danoff-Burg, 2000), and emotional intelligence (Schuette, Malouff, Hall, Haggerty, Cooper, Golden, \& Dornheim, 1998). Based on the demonstrated differences in the outcome of emotional intelligence and PTSD symptom expression between men and women, examining whether these differences hold up in field EMTs as well are important so that resulting outcomes may be used to determine treatment needs for this population.

\section{Attachment}

Attachment was proposed by Bowlby $(1973,1982)$ as the style of behavior by which humans connect with other human beings (Mikulincer, Solomon, Shaver, and Ein-Dor, 2014); with the two primary styles of attachment being anxious attachment and avoidance attachment (Brennan, Clark, \& Shaver, 1998). Anxious attachment has been described as "the degree to 
which a person worries that others will not be available in times of need" (Mikulincer, Solomon, Shaver, and Ein-Dor, 2014, p. 209), and avoidant attachment has been described as "the extent to which a person inhibits support-seeking and relies on him/herself as the sole source of safety" (Mikulincer, Solomon, Shaver, and Ein-Dor, 2014, p. 209). Brennan, Clark, and Shaver (1998) assert that those who score low on both of these attachment dimension may be said to be securely attached, whereas those who score high on both of these dimensions exhibit insecure attachment. Attachment style has been suggested to be related to the development of traumarelated symptoms, with child abuse being attributed to result in insecure attachment styles (Alexander, 1992; Muller, Sicoli, \& Lemieux, 2000), as has family functioning and maternal depression (O’Connor, \& Elklit, 2008).

Mikulincer, Solomon, Shaver, and Ein-Dor (2014) propose that having secure attachment leads to resilience when one encounters stressful situations, reporting that those who have "a sense of being loved and supported by significant others is a source of strength and resilience during times of stress" (Mikulincer, Solomon, Shaver, \& Ein-Dor, 2014, p. 210). Their study demonstrated a need for stable security attachments in the healing of PTSD, and that without the benefit of healthy and attachment security, POW's were unable to heal from PTSD (Mikulincer, Solomon, Shaver, \& Ein-Dor, 2014).

In a study on attachment and psychological symptom expression, individuals who endorsed greater levels of attachment anxiety also reported greater levels of psychological symptom expression (Elwood \& Williams, 2007). Elwood and Williams (2007) also found associations between PTSD-related cognitions, which include "negative cognitions about the self, negative cognitions about the world, and self-blame for the trauma," (Elwood \& Williams, 2007, p. 1190) and the development of both PTSD and depressive symptoms. Individuals with 
attachment avoidance and attachment anxiety demonstrated greater levels of PTSD in a study by Busuito, Huth-Bocks, and Puro (2014) as well. They found that when avoidant romantic attachment was present in relationships, previous childhood abuse was shown to result in PTSD symptom expression (Busuito, Huth-Bocks, \& Puro, 2014). In this study, romantic attachment style acted as a mediator between previous traumatic events and PTSD symptom expression.

Clark and Owens (2012) found the same relationship between anxious attachment in romantic relationships and PTSD symptom severity in U.S. veterans of Iraq and Afghanistan. They report that secure relationship attachment style is a protective factor for this population (Clark \& Owens, 2012). Anxiety, depression, negative affect, low levels of emotional adjustment, and alcohol use has been associated with insecure adult attachment, with the assumption being that individuals who feel secure are able to connect with others, to be trustworthy, responsive, and lovable with others, while those who are insecurely attached do not have these capabilities due to constant negative thoughts (mental rumination), negative affect, and negative memories (Declercq, \& Willemsen, 2006). A meta-analysis performed by Woodhouse, Ayers, and Field (2015) once again confirmed the association between insecure attachment and PTSD symptoms.

Multiple other studies with various populations demonstrate the same connection between anxious and insecure attachment and PTSD symptomatology, including civilians in Southern Israel exposed to terrorist attacks (Besser, Neria, \& Haynes, 2009), women with gynecological cancer (Holt, Jensen, Hansen, Elklit, \& Mogensen, 2016), individuals with chronic whiplash-associated disorder (Andersen, Elklit, \& Vase, 2011), Vietnam combat veterans (Nye, Katzman, Bell, Kilpatrick, Brainard, \& Haaland, 2008), university staff and students (Arikan, Stopa, Carnelley, \& Karl, 2016), civilians exposed to the Israel-Gaza war in 2009 (Avi, \& Yuval, 
2010), security guards (Bogaerts, Daalder, Van Der Knaap, Kunst, \& Buschman, 2009), female healthcare workers in a longitudinal study on violence and health (La Flair, Bradshaw, Mendelson, \& Campbell, 2015), and school children following a school-shooting trauma in Finland (Turunen, Haravouri, Punamaki, Suomalainen, \& Marttunen, 2014). Ein-Dor, Doron, Mikulincer, Solomon, \& Shaver (2010) found that this connection was significant enough that it affected not only the individual exposed to the traumatic event, but to their romantic partner as well in relation to whether that individual developed Secondary Stress Trauma (STS) or not. Benoit, Bouthillier, Moss, Rousseau, and Brunet (2010) propose that it is the individual's attachment, whether that be insecure or secure, that comes into play when an individual is exposed to a threatening or stressful situation.

\section{Resilience}

Resilience has been defined and conceptualized in several different ways. Oginska-Bulik and Kobylarczyk (2014) make the distinction between resilience and resiliency, proposing that resiliency is more of a characteristic or personality trait that individuals possess, and that resilience is more of a process. This has been proposed by other researchers as well (Yehuda \& Flory, 2007), stemming from the debate that is present in most research on human behavior; whether the variable being studies is a state or trait, or a combination of both. Resilience has also been defined as the ability to bounce back after a traumatic event (Yehuda \& Flory, 2007), as being a "concept of personal hardiness" (Kuiper, 2012, p. 475), or the "individual ability to separate from unpleasant life events" (Oginska-Bulik \& Kobylarczyk, 2015, p. 708). Still others propose that resilience is the ability on the part of first responders to withstand trauma (Chang \& Taormina, 2011), and "the ability to rebound from and positively adapt to significant stressors: (Sinclair \& Wallston, 2004, p. 94). 
Research on resilience has focused on both the effects of resilience in different situations and circumstances, as well as with regard to the components that make up resiliency. Yehuda and Flory (2007) assert that prior to the conceptualization of PTSD as a diagnosis in the DSMIII, resiliency was considered to be a fundamental component of the individual, with those who were considered to be "resilient" demonstrating the ability to bounce back after traumatic and adverse life experiences, while those who didn't being considered to be lacking in resiliency. This lack of resiliency, therefore, was considered to be a character flaw for which people were severely stigmatized (Yehuda \& Flory, 2007). Once PTSD was established as a recognized diagnosis in 1980, the door was opened for examination of the variables that factored into the expression and severity of its symptoms (Yehuda \& Flory, 2007).

Some of the questions that have been examined in relation to resiliency over the years have included the examination of resiliency as a psychopathological component and resiliency as a component of vulnerability (Yehuda \& Flory, 2007, p. 439). Richardson (2002) proposed a metatheory on resilience in which it was proposed that the research on resilience has followed three primary avenues: 1) the process of resiliency as developed by those individuals who have faced adversity; 2) the characteristics of those individuals; and 3) the cognitive mechanisms that accompany the development of resiliency for the individual. Some of the factors that have been examined have included an examination of the type of trauma exposure, prior history of trauma exposure, personality characteristics, genetics and neurobiological factors, gender, age, childhood factors, affect regulation, ego defenses, cognitive factors, and social support systems (Wilson, 1995; Zeidner \& Endler, 1996; Zuckerman, 1999; Frederickson, 2002; Agaibi, 2003; Schore, 2003; Southwick, Morgan, Vythilingham, Krystal, \& Charney, 2004). 
Resilience has been associated with post-traumatic growth (Vera, 2013; Oginska-Bulik and Kobylarczyk, 2014), with positive emotions (Frederickson, 1998; Frederickson, 2001), greater levels of physical wellness (Chang \& Taormina, 2011), higher levels of satisfaction with personal relationships, medical care, and community life (Chang \& Taormina, 2011), and lower levels of burnout (Chang \& Taormina, 2011). It has also been found to be a contributing factor in the determination of perceived levels of stress (Mroz, 2015), and it has been found to serve as a buffer against depressive symptoms (Sinclair \& Wallston, 2004).

According to a review performed by Agaibi and Wilson (2005), seven factors have been identified that appear to be associated with the development of resilience as reported by Raphael (1993) and Wilson (1995). These were:

(a) locus of control (i.e., a sense of efficacy and determination, (b) self-disclosure of the trauma experience to significant others, (c) a sense of group identify and sense of self as a positive survivor, (d) the perception of personal and social resources to aid in coping in the posttrauma recovery environment, (e) altruistic or prosocial behaviors, (f) the capacity to find meaning in the traumatic experience and left afterward, and $(\mathrm{g})$ connection, bonding, and social interaction within a significant community of friends and fellow survivors. (Agaibi \& Wilson, 2005, p. 203)

\section{Vulnerability to Developing PTSD}

Avoidance factors can often be shown to mediate trauma and PTSD, with indications that one, preceding the other, may be a predictor. Determining vulnerability to develop PTSD has been elusive to research even with considerable time spent on the subject. Personality factors, environmental factors, and biological factors are all thought to play a role. While personality factors that increase anxiety sensitivity are often thought to precipitate PTSD development, another factor, experiential avoidance, has been found to mediate between trauma exposure and the development of PTSD. Specifically, the greater the level of experiential avoidance and posttraumatic exposure, the more likely individuals were to develop PTSD (Maack, Tull, \& Gratz 
2012). This was true for individuals who experienced trauma in sexual assault, vehicular accidents, and combat (Maack, Tull, \& Gratz 2012). In fact, the role of avoidance has been implicated in not only mediating between exposure and development, but it has also been implicated as a factor in maintaining PTSD symptomology (Maack, Tull, \& Gratz 2012).

Avoidance is a maladaptive control strategy that prevents disconfirmation of negative appraisals, resulting in the maintenance of a perceived current threat (Fleurkens, Rinck, \& Minnen 2014). In line with this, trauma-focused treatments stress the role of avoidance in the maintenance of PTSD (Fleurkens, Rinck, \& Minnen 2014). Prolonged exposure to safe, but anxiety-provoking trauma-related stimuli is considered a treatment of choice for PTSD (Fleurkens, Rinck, \& Minnen 2014). While individuals may be aware of the explicit avoidance that they adopt, they may not be aware of the implicit avoidance to such factors as remembering events or thoughts about the trauma (Fleurkens, Rinck, \& Minnen 2014). The Fleurkens, Rinck, and Minnen (2014) study did indicate, however, that the level of threat was also a predictor of implicit avoidance.

It has been postulated that individuals who experience trauma should receive some form of treatment within the first three months after experiencing trauma (Vashdi, Bamberger, Bacharach, 2012). While the recommendations have become almost universal in larger countries, time and again, studies show that many individuals do not seek this treatment until more severe symptoms have been experienced over longer periods of time (Vashdi, et. al, 2012). Of course, as studies have shown, delays in seeking treatment can be costly to both the individuals and the organizations to which they belong to or work with (Vashdi, et. al, 2012). This can also lead to other pathology such as depression, anxiety, and substance use disorders (Vashdi, et. al, 2012). The challenges associated with encouraging individuals to seek help has 
been demonstrated across multiple trauma types, occupations, and gender. Understanding what factors predict the delay or even rejection of help may allow researchers and clinicians to develop procedures and education materials that will encourage individuals to seek help.

In recent decades, peritraumatic dissociation during or immediately after a traumatic event has been considered to be a particularly important risk factor for the development of posttraumatic stress symptoms and PTSD. The concept of peritraumatic dissociation refers to experiencing changes or distortions in the perception of time, space, and the self, during or immediately after a traumatic event (Kumpula, Orcutt, Bardeen, \& Varkovitzky, 2006). While the study by Kumpula et al (2006) did not demonstrate enough statistical significance for this to be of value, it does demonstrate that future research into this area could prove beneficial.

Other studies have focused on the vulnerabilities of individuals who work in occupations that experience high stress and critical incidents such as police officers (Marchand, Boyer, Nadeau, Beaulieu-Prévost, \& Martin, 2015). Pre-traumatic exposure factors such as cumulative exposures, limited field experience, past traumatic events, and the presence of mental health disorders in family members (Marchand, et al, 2015). Peritraumatic factors such as physical and emotional reactions during trauma, a threat to one's life partner or physical integrity, severity of exposure, as well as exposure to death constitute risk factors in the development of PTSD (Marchand, et al, 2015).

Post-traumatic factors that may increase the likelihood of developing PTSD may include factors such as short periods of time allocated by others to recover from the event, dissatisfaction with one's support system, or the lack of social support outside of the incident-related situation (Marchand, Boyer, Nadeau, Beaulieu-Prévost, \& Martin, 2015). Incident-related physical injuries, depressive symptoms, as well as subsequent negative life events (Marchand, et al, 
2015). The study by Marchand et al (2015) did support the fact that risk factors can be predictive of PTSD development; however, strong support factors can prevent this as well. The fact that first responders experience all of these factors is important in understanding both the nature of the job, but, also how avoidance plays a role in treatment resistance and rejection Marchand, et al, 2015).

\section{Possible Contributing Factors}

Police officers, firefighters, and emergency services personnel may be repeatedly exposed to situations involving fatal accidents, crime, child abuse, homicide, suicide, rape, and other traumatic events as part of their usual duties. Some studies have looked at the coping styles of first responders and have demonstrated that avoidance is seen as a mediator of PTSD symptoms (LeBlanc, Regehr, Jelley \& Barath, 2008). Studies of emergency responders and armed forces personnel exposed to traumatic events have found an association between negative coping styles and psychological distress (LeBlanc, Regehr, Jelley \& Barath, 2008). For instance, in emergency health care workers, the use of wishful thinking, comfort eating, drinking, smoking, and avoidance are associated with reported distress levels (LeBlanc, Regehr, Jelley \& Barath, 2008). The use of avoidant coping styles, as well as emotional ones, again demonstrated a higher distress level for these individuals (LeBlanc, Regehr, Jelley \& Barath, 2008). Avoidance, as a coping skill, while common for those who are experiencing the effects of trauma, more likely than not will increase the symptoms felt even when that is the opposite of what the individual is trying to achieve. Further, it is possible that the coping styles of EMS workers may have an impact on the relationship and health of the spouses of these workers. 


\section{Treatment of PTSD}

Treatment of PTSD has included Cognitive Behavioral Therapy (CBT) to assist in dealing with symptoms of hyperarousal, negative cognitions, and avoidant behaviors (AntunesAlves \& Comeau, 2014), as well as in using conditional response treatments similar to the process used by Pavlov, in which a conditioned stimulus (CS), such as the fear response, being paired with an unconditioned stimulus (US), such as mild shocks, thereby resulting in the successful association of the US with the CS, and extinguishing the undesirable fear response (Singewald, Schmuckermair, Whittle, Holmes, \& Resler, 2014). Pharmacological agents have also been used in an attempt to extinguish the learned fear response through the use of benzodiazepines (anxiolytics), as are used primarily as "complementary techniques; electrophysiology, immediate early gene mapping, tracing studies, lesioning/inactivation approaches, and optogenetics)" (Singewald, Schmuckermair, Whittle, Holmes, \& Resler, 2014, p. 4). Finally, family therapy has been shown to be useful in assisting families in dealing with PTSD (Dekel \& Monson, 2010). However, specified treatment that has proven to be efficacious across the board for all individuals experiencing PTSD has not been identified (Forneris, Gartlehner, Brownley, Gaynes, Sonis, Coker-Schwimmer, Jonas, Greenblatt, Wilkins, Woodell, \& Lohr, 2013), although Eye Movement Desensitization and Reprocessing (EMDR) has shown great promise in treating those with PTSD (Thomaes, Engelhard, Sijbrandij, Cath, \& Van den Heuvel, 2016).

\section{Epidemiology and Prevalence Rates of PTSD}

Studies have documented that female spouses are at a greater risk for PTSD than are male spouses (Kubzansky, Koenen, Jones, \& Eaton, 2009; Tolin \& Foa, 2006). According to research on Dutch couples shows that this risk factor may include increased risk for women due to 
aggression being the means of dealing with their own PTSD symptoms, as well as a general overall increase in risk of PTSD for female spouses, with increased PTSD found in women with spouses demonstrating more severe PTSD symptomatology (Bramsen, van der Ploeg, \& Twisk, 2002). Dekel and Monson (2010) assert that the increased risk of PTSD for women may possibly be due to the greater sense of caregiver burden that is expected of women than is expected of men, as well as due to the increased empathy that women demonstrate than men (Zaki, 2014). Current estimates show that approximately $12 \%$ of the American population will develop PTSD at some point in their life, with multiple courses of PTSD, each lasting at least two to three years (Kessler, 2000 as cited in Galovski \& Lyons, 2004).

\section{Health and Related Risks of PTSD}

Health risks of PTSD include increased risk of Coronary Heart Disease (Caska, Smith, Renshaw, Allen, Uchino, \& Carlisle, 2014; Kubzansky, Koenen, Jones, \& Eaton, 2009), greater job loss (Brackbill, Hadler, DiGrande, Ekenga, Farfel, Friedman, Perlman, Stellman, Walker, Wu, Yu, \& Thorpe, 2009), unemployment at an increased rate of 150\% (Galovski \& Lyons, 2004), persistent and long-term mental health conditions (Brackbill, Hadler, DiGrande, Ekenga, Farfel, Friedman, Perlman, Stellman, Walker, Wu, Yu, \& Thorpe, 2009), increased marital distress and difficulties in intimate relationships (Caska, Smith, Renshaw, Allen, Uchino, \& Carlisle, 2014), and marital instability at an increased rate of 60\% (Galovski \& Lyons, 2004). Family adjustment is also impacted, as well as marital intimacy, and violence against women has been shown to be at much higher rates in men who have been diagnosed with PTSD (Monson, Taft, \& Fredman, 2009). Rates of divorce increase exponentially, as does the risk of developing PTSD and STS to wives of men with PTSD (Monson, Taft, \& Fredman, 2009). 


\section{Protective Factors}

Several researchers have examined factors that appear to be protective in the development of PTSD symptoms following exposure to a traumatic event. Social support appears to be one of these protective factors, with social support that is tangible to the individual, and support that makes the individual feel as though they belong within a group of individuals (most often family) having the greatest impact against the development of PTSD (Dineberg, Mccaslin, Bates, \& Cohen, 2014). Both Kaniasty \& Norris (1993), and Frye (1995) found the same protective impact in relation to individuals with severe disaster exposure individuals as well as individuals from the Hmong culture, and the importance of the kinship support system as having a negative correlation with the development of PTSD symptomatology. The use of spiritual coping has also shown to be protective as a factor against developing PTSD symptoms (Bryant-Davis \& Wong, 2013), as has involving family members in the treatment of PTSD (Monson, Taft, \& Fredman, 2009).

\section{Significance of the Topic}

While PTSD has been a long-researched area, both in the medical and the mental health fields, research has primarily been focused on PTSD primarily as it relates to combat veterans and those directly and actively in military service. Research has also demonstrated the effects of PTSD in other male dominated careers, such as with police officers and firefighters. However, only one research study was identified with regard to PTSD in field EMTs. Morrison (2014) reflects perfectly the general consensus in field EMTs when she reports that the general population has a lack of understanding regarding the work that EMTs do, nor their qualifications or work experiences. EMTs are not only often referred to as "ambulance drivers," but when the public becomes aware of events which require action by first responders, EMTs are often 
omitted from acknowledgement unless they work for the fire service. Most often, police officers, firefighters, and paramedics on the fire department are noted, while EMTs and paramedics in the private sector are failed to be identified as those valued individuals who have served in this important capacity.

Police, firefighters, paramedics, emergency medical technicians (EMTs), and similar responders often face traumatic events on a regular basis. Not only do they respond to traumatic events such as vehicle accidents, but they also face loss of life for colleagues, and the threat of loss of life for themselves and others. Their work entails situations that they have no control over due to the fact that they never know what type of a call they will get, what the situation will be when they arrive, whether there will be a threat to their safety or their life, nor what type of trauma they may see or have to deal with. It is not uncommon for EMTs to arrive on scene and have to wait for law enforcement to clear the scene, for example, in domestic violence situations, before they are allowed to move in and do the job for which they have been trained.

Vogel, Cohen, Habib, and Massey (2004) state it perfectly when they assert that “emergency medical services (EMS) personnel often are both care providers and themselves at risk" (p. 35). It has been shown that individuals most often use emotion-focused coping when faced with situations which they assess they are not able to control (Novara, Garro, \& Dr Rienzo, 2015). Because emotion-focused coping has been shown to be associated with greater levels of PTSD symptom expression, the unknowingness of the field is a significant factor to consider in the treatment of those who work under such conditions. This continued and persistent focus on the possibility of threat wreaks detrimental effects on the nervous system. Hart (2003) notes the severity of this type of forced hypervigilance when he reports that the "human nervous system is not designed for protracted battle" (p. 2), stating that this prolonged exposure to threat results in 
“the emotional biostat burning out sooner or later - no one's neurological wiring comes equipped with 100\% insulation" (p. 2).

With the previously discussed outcomes of PTSD on functioning and health, such as those seen neurologically with changes in the neurological response system, psychologically, such as the increased rates of depression, and in terms of an individual's physical heath, the detriment of being a population of trauma exposed individuals leaves the recognition of the severity of traumatic events could greatly impact not only the outcomes of that trauma on the individual, but also the ability of the individual to receive adequate, appropriate, and sufficient services to deal with the impact of the traumatic stressor. Evaluating the impact of coping skills, emotional intelligence, attachment, and resiliency as covariates on PTSD symptom severity and expression for private sector EMTs will lend a greater understanding as to the outcomes of PTSD on those in the field who may not be as identified or recognized as experiencers of traumatic events.

\section{Summary}

The focus of this chapter was on a review of the literature as relevant to the constructs of interest within this research study. More specifically, this literature review examined factors related to PTSD, emotional intelligence, attachment, coping, and resiliency as relevant to the EMS field, with the reader being provided with conceptual definitions for each, as well as the foundation of previous research in each of these areas. This review has resulted in the assertion that the four independent variables (emotional intelligence, attachment, coping, and resiliency) may be important factors as relevant to and that contribute to PTSD symptom expression in private sector EMTs. The evaluation of these factors in relation to PTSD within this sector of the field is important as there have been less than a handful of studies examining PTSD in private 
sector EMTs as impacted by emotional intelligence, attachment, coping, and resiliency as a group of factors together. The results of this study will be important, as they may provide insight into factors unique to the private sector of the EMS field and needs directly related to this population of emergency providers. In this, then, this review has identified a gap in the research that needs to be identified and filled, and will, therefore, contribute to the knowledge in the field regarding this rarely researched population. 


\section{CHAPTER 3}

\section{METHODOLOGY}

\section{Introduction}

\section{The Purpose of the Study}

The purpose of this study was to examine the relationship between coping skills, emotional intelligence, attachment, and resilience on PTSD symptom expression in private sector Emergency Medical Technicians (EMTs) workers. While PTSD has been very well researched, most of the research has focused on military populations, military veterans, and more commonly identified first responders such as firefighters and police officers. Because research on EMTs have been less well-defined, with most of the research on EMTs being with those who work in the public sector, most often with fire service, research on private sector EMTs has been lacking. The hole that remained in PTSD research on private sector EMTs provided the rationale, base, and demonstration of the need for the current study.

\section{Organization of the Chapter}

This chapter began by addressing the purpose of the study, and reintroduced the research questions for the study. The research design was also presented, as well as the population and sample for this study. The instrumentation that was used for this study was expressed, as was the procedure that the study utilized. How the data was obtained and what the process consisted of in terms of ensuring the appropriateness of the evaluative process statistically was discussed next, with a discussion of the analysis of the data. Finally, a summary of this information was presented to the reader. 


\section{Research Questions}

\section{Qualitative Interview Questions}

The questions for the individual interviews during the qualitative portion of the study were as follows:

1. Describe your work environment as an EMT working in the private sector of the field.

2. What were some of the things that drew you to desire working in this field?

3. Describe what brought you to working in the private sector versus the public sector of the field.

4. In your opinion, what are the differences between working in the private versus the public sector of the field?

5. How does the EMS culture play a role in your experiences as a private sector EMT?

6. In your opinion, is there a difference in community or societal response to you as a private sector EMT versus a public sector EMT? If so, how does the public's response to your work in the private sector impact your work as a private sector EMT?

7. What advice would you give to someone who is considering working in the private sector of the field?

\section{Quantitative Research Questions}

Following were the research questions for the quantitative portion of this study:

1. What is the severity of PTSD among private sector EMTs?

2. Are there differences in the severity of PTSD among EMT-B's, EMT-I's, and EMTP's? 
3. What coping strategies do private EMTs use?

4. What are their levels of emotional intelligence, resilience, and attachment?

5. What is the nature of the relationship between PTSD severity and the following variables: emotional intelligence, coping skills, resiliency, and attachment?

\section{Qualitative Research Design}

Beyond examining research information related to the variables of interest as related to PTSD symptoms in private sector field EMTs, it was important to identify differences between private and public sector EMTs, as this assists in identifying qualitative differences that demonstrate how PTSD may be experienced differently between the two groups of EMTs when both of those groups meet the same diagnostic criteria for PTSD. Therefore, a qualitative design was also implemented, making the full design for this study a mixed-methods design. The qualitative portion of the study used a combination of methods, which utilized individual interviews in phenomenology.

Phenomenology has commonly been used as a venue by which the researcher is able to come to an understanding of the lived experiences of the participants (Creswell, 1998; Dahl, \& Boss, 2005; Crosbie-Burnett, Lewis, Sullivan, Podolsky, Mantilla de Souza, \& Mitrani, 2005). Van Manen (1990a) makes the assertion that it is within the narrative of the individual that the definition of the "essences of their lived experience" may be found (p. 10). "Phenomenology seeks to examine the experiences of participants, since similar experiences could mean different things to different people" (Orozco Markert-Green, 2009, p. 24). Identifying those experiences that were unique to private sector EMTs were sought in an attempt to provide a greater richness and depth into the experiences of private sector EMTs as compared to the experiences of PTSD in the public sector as previously defined in the literature. 
Because diagnostic evaluation simply alerts one to the fact that an individual either meets or does not meet criteria for a diagnosis of PTSD, it is through the use of individual interviews that the differences regarding how PTSD is experienced by private sector EMTs may be revealed. The qualitative portion of the research study was based on an attempt to identify what the experiences of EMTs working in the private sector of the field were, with the intent being to "provide for a richness of information, and to give voice to a population that may not have been given that voice before (Orozco Markert-Green, 2009, p. 24).

In addition to the identification of variables as related to the expression of PTSD symptoms, and the identification of the uniqueness's as inherent in the experiences of private sector EMTs. This provided for a more rich and in-depth process, in which information not contained in the quantitative portion of the study would become available to this researcher, thus granting information as to the lived experiences of private sector EMTs. According to Flick (2006), qualitative research is research that "utilizes a multimethod framework as its focus, so that it may obtain the most in-depth understanding of the phenomena that is the subject of research" (Orozco Markert-Green, 2009).

\section{Qualitative Objective}

The objective of the qualitative portion of this study was to identify the experiences of EMTs working in the private sector of the field to evaluate for three factors: 1) Whether there were perceived differences between working in the private versus the public sector of the field;

2) If private sector EMTs express similarities in the role EMS culture plays in their experiences as private sector EMTs; and 3) Whether private sector EMTs express perceived differences in community or societal response to their work as opposed to community or societal response to the work of public sector EMTs. Because the quantitative portion of the study addressed the 
component related to trauma through the examination of PTSD, questions related to trauma were not addressed in this section.

\section{Qualitative Background and Foundation}

The qualitative portion of this study had its foundations in several areas. The interest for this study overall began with experiences in this researcher's private life as based on this researcher's experiences in the lack of recognition and attention provided to the private sector of the field, as well as the experiences of other field professionals known personally to this researcher. Drawing on these experiences and information, as well as on a review of the literature confirming the lack of research devoted to private sector EMTs, the quantitative portion of the research study was initiated. Once the quantitative data became available and demonstrated the need for additional and more in-depth information regarding this population, the qualitative portion of the study went forward. Previous to the initiation of the research gathering, a total of seven questions were selected for this portion of the study based on the research questions for the quantitative portion of the study that, just as with public sector EMTs, there would be a demonstration of PTSD symptom expression in private sector EMTs, and that symptom severity would demonstrate relationships with the four variables: coping skills, emotional intelligence, levels of attachment, and resiliency.

\section{Qualitative Validity}

When examining validity in qualitative research, one must identify whether or not the information obtained is true with regard to the population of interest and with regard to the information obtained. According to Cypress (2017), the standards for reliability and validity in qualitative research was set by Guba and Lincoln (1989) with their proposal of four guidelines. These consisted of what they termed credibility, transferability, dependability, and confirmability 
(Lincoln \& Guba, 1989). Credibility refers to the establishment of results that are credible from the perspective of the participant, and is analogous to internal validity in quantitative research (Trochim, 2020). Transferability, which is analogous to external validity in quantitative research refers to the generalizability of the results (Trochim, 2020). Dependability, which is analogous to reliability, refers to the ability to replicate or repeat the results of the study, with the researcher being responsible for describing how changes may occur within the setting of the particular research context that could affect how future research may be approached in replication studies (Trochim, 2020). Finally, confirmability, which is analogous to objectivity, refers to the ability for others to corroborate or to confirm the research results (Trochim, 2020).

Other researchers propose that rigor, or the "quality or state of being very exact, careful, or thorough and accurate" (Oxford English Dictionary, 2013 as cited in Cypress, 2017, p. 254). Trustworthiness is another aspect that is considered in qualitative research, and is considered to be the authenticity, quality, and truthfulness of the research findings, and is related to the amount of confidence or degree of trust that the readers of those research findings may have in the results (Cypress, 2017). In addition to these, validity is of concern in qualitative research, just as it is in quantitative research, with validity being concerned with both the accuracy of the research findings as well as the truthfulness of those findings (Van Manen, 1990b as cited in Cypress, 2017). Because qualitative research seeks to understand the phenomena of interest as expressed from the viewpoint of the participant without any manipulation of that phenomena, the data is grounded in that process. This assists in providing both reliability and validity, as this lends itself to the rigor of the study process and findings (Cypress, 2017). Smith (1989) made the argument that it is this expression of the viewpoint of the participant that lends itself to the truth 
of that individual's experience or trustworthiness, which then becomes a social agreement, and therefore, reliable and valid for that experience.

Validity within this study was achieved through the use of researcher/participant consensus with regard to the information as provided by the participants. In other words, this researcher verified the information as presented by each participant and what the definition of the terminology entailed as used by each participant without identifying this information in a specific manner to be used later in the coding process by first verifying the accuracy of the terminology with each of the participants. By allowing each participant to be a part of the formulation of constructs related to their experiences, construct validity was ensured, which then led to the formulation and use of quality criteria in being able to both explore and to describe the criteria that was most relevant to each of the research participants. By default, this then also ensured rich and quality constructs and quality criteria for the study itself. Because of this process, trustworthiness was demonstrated through the use of each of the combination of each of these components together, without placing any personal meaning or interpretation of this researcher on each of the terms, concepts, and constructs, but instead, the reliance of each of these was used in such a way as to permit for the information obtained from the participants to fit them and their experiences.

\section{Quantitative Research Design}

To address the relationship between PTSD in private sector EMS workers and the independent variables (a) coping, (b) emotional intelligence, (c) attachment, and (d) resiliency, survey research was used, followed by a correlational analysis of the results. The relationship between PTSD symptom expression in EMS workers and the four independent variables (coping, emotional intelligence, attachment, and resiliency) was examined using a quantitative approach 
to obtain the data, with questionnaires serving as the means by which the information was gathered.

Babbie (2010) defines quantitative research as the process by which the testing of objective theories is utilized in order to examine the relationships between variables. The measurement of identified variables was performed in order to obtain numerical data that was then analyzed using statistical methods. Information obtained provided pertinent data relevant to the expression of PTSD in populations of EMS workers, with statistical sampling of subpopulations of EMS workers used for the generalization of the data to be inclusive of all EMS workers. A quantitative design was chosen to be the most relevant and suitable to answer the research questions related to PTSD symptom expression in private sector EMS workers.

A non-experimental research design was chosen over an experimental design, as the goal of this research was to identify the relationships between the variables, rather than to utilize a specified treatment and measurement approach. Non-experimental designs are described as research that (Babbie, 2010; Muijs, 2010) is descriptive in nature, whereas experimental designs traditionally measure a dependent variable before and after treatment. McMillan \& Schumacher (2010) report the non-experimental design process as involving a description of specific phenomena and the evaluation and examination of the relationships that exist between a set of variables prior to any manipulation of those variables, as one would see in experimental designs. Survey research was the type of non-experimental design used for this study. Hager, Wilson, Pollak, and Rooney (2003) state:

The survey research method is geared toward comparing the same information for a large number of cases. The general approach involves defining a population of cases that are the topic of study, creating a list of those cases (the sampling frame), selecting a random 
sample of those cases, and collecting data on the sampled cases. Adherents to this method hold that random selection of cases from the sampling frame provides the best chance that the selected cases will be representative of the population. To the extent that the selected cases are representative, researchers can validly infer characteristics of the larger population based on the characteristics of the sample. (p. 252)

A common method of obtaining this relational information is through the use of a correlational design, in which the research is performed in the same manner as described above (Grajales, 2013). Identification of relationship between variables (DV and IV's) was the goal of this research study, with implications for further research and clinical treatment to follow.

The purpose behind the use of survey research was multiple. First, survey research allowed for a more extensive sampling of the population of interest. Second, survey research provided for a cost effective means of obtaining information from many more respondents than would have been possible through the use of either phone interviews or paper surveys. Third, survey research enabled the researcher to obtain information from respondents in remote locations. Fourth, survey research allowed respondents to answer more fully and accurately due to respondents tending to demonstrate more open and honest answers than when research is performed in more traditional ways (DeFranzo, 2012). DeFranzo (2012) asserts that respondents provide less ambiguous and more honest responses when response methods are utilized that allow for anonymity. Fifth, surveys are able to describe characteristics of larger populations, thus ensuring a more accurate sample from which more specified results may be obtained. This, then, enabled the researcher to draw more specified and accurate conclusions, and resulted in a greater ability to make decisions related to that specific population in order to facilitate treatment changes and/or decisions relative to the population of interest. Finally, because the goal of this 
research study was to provide a baseline view of PTSD in private sector EMTs, survey research assisted in identify how and whether PTSD is experienced by private sector EMTs in order to formulate the basis and grounding for further research on this population with regard to PTSD experience and symptom expression.

The use of questionnaires provided for respondents from anywhere within the United States to be included in this research study without having to live in close proximity to this researcher and it allowed for an examination of these variables in a manner that provided for greater geographical generalization. Further, the absence of a researcher asking questions in the physical proximity of the respondent may possibly have resulted in respondents being able to respond in a more honest and forthright manner in their responses, without the effect of perceived judgement or bias present in the gathering of information from participants. This method also contained the benefit of lower cost for the information gathering process and ease of use for both this researcher and for the participants. However, the lack of face-to-face interaction disallowed personal interaction between this researcher and participants, which may possibly have resulted in the study feeling more "clinical" and impersonal than a one-on-one gathering of information process.

\section{Population and Sample}

\section{Population}

According to the National Institute for Occupational Safety and Health (NIOSH) and the Centers for Disease Control (CDC), there were 826,000 licensed and credentialed EMS workers in the United States as reported through the 2011 National EMS Assessment Report (2011), and includes both volunteer and paid workers. The sample for this study consisted of Emergency Medical Providers residing and working in the United States of America as sampled from 
January through June of 2018. The sample groups that were studied include EMS providers in the field (outside of the hospital system) employed with private ambulance companies. Although the focus was solely on Emergency Medical Technicians (EMTs), these were represented by EMT-Basic's (EMT-B's), EMT-Intermediates (EMT-I's), and EMT-Paramedics (EMT-P's).

The reason for this was based upon the hypothesis that the differences in scope of practice, which differs based on the certification level of the EMT (EMT-B; EMT-I; EMT-P), as well as from region to region and rural versus urban settings, would have an impact on the severity of calls that the EMT will be responding to, and therefore, the exposure to events that could lead to PTSD. Examination of coping, emotional intelligence, and attachment may show differences in impact on the development and symptom expression of PTSD for those working in the field within the private sector; therefore, it was necessary to break down the participants based on these categories in order to identify the true impact of coping, emotional intelligence, and attachment on PTSD symptom severity.

\section{Sample}

The sample size for this study was estimated through power analysis, with a total estimated sample size of 300 participants, to include each of the three certification levels; EMTB's, EMT-I's, and EMT-P's. It was anticipated that the pool of EMTs would be generated from EMTs throughout the United States, thereby providing a sufficient pool of EMTs from which 300 would have been able to be selected. However, in the gathering of this data, only 113 responses were received, with 57 responses by public sector EMTs and 56 responses by private sector EMTs, 3 outliers, and 3 incomplete responses, leaving only 50 qualified respondents; therefore, the sample became a sample of convenience at that point, disallowing the previous goal of randomly selecting 300 total participants as represented by each of the three certification 
levels (EMT-B, EMT-I, and EMT-P) as a whole. Attempts to gather additional EMTs included sending the Survey Monkey link directly to EMT organizations throughout the United States in an effort to gather a sufficient pool to allow for random sampling, as described above. See Table 2 below for the power analysis:

Table 2

Power Analysis

\begin{tabular}{lllll}
\hline Statistical Test & Sample Size & Power & Correlation & $\begin{array}{l}\text { Significance } \\
\text { Level }\end{array}$ \\
\hline Correlation & 28.870 & 0.800 & 0.5 & 0.05 \\
Correlation & 84.750 & 0.800 & 0.3 & 0.05 \\
Correlation & 782.450 & 0.800 & 0.1 & 0.05 \\
Correlation & 300.000 & 1.000 & 0.5 & 0.05 \\
\hline
\end{tabular}

Sampling took place through the following means: A convenience sample was drawn from EMTs in the United States who work in the private sector. The sample was obtained through social media sites such as Facebook, by word of mouth, and by advertising through The State of Michigan Department of Health and Human Services (DHHS). The Survey Monkey link was placed directly on this researcher's Facebook page, allowing for any EMT to be able to take the survey. Additionally, the link was placed by friends on their Facebook pages, as well as friends who are EMTs placing it on their Facebook pages and printing it out and placing it as their places of employment. The State of Michigan Department of Health and Human Services placed the link in their newsletter on a weekly basis.

Participants were selected based on the following eligibility criteria: Eligible participants were identified as being: 1) be an individual who works in the Emergency Medical Services field as a private sector EMT; 2) a private sector field EMT who has been exposed to traumatic events as a result of their job description and working conditions; or 3) an individual who has worked in 
the private sector as an EMT within the previous 5 years, and who had been exposed to traumatic events as a result of their job description and working conditions. Exclusion criteria included 1) individuals who are first responders, but who have never worked in the private sector of the EMS field; 2) individuals who are first responders and have worked in the private sector of the EMS field, but not within the previous 5 years or who are working in the public sector currently; and 3) individuals who are first responders, but do not currently, nor have they ever, worked in the private sector.

Participants completed survey instruments though SurveyMonkey, an online survey software company. Participants were provided access to study questionnaires and materials on the SurveyMonkey website, after which all participants were entered into a confidential database by SurveyMonkey, with participant numbers generated as each potential participant was entered. The SPSS database was sent to this researcher from SurveyMonkey, after which statistical analyses were performed by this researcher.

\section{Variables}

The variables used in the quantitative portion of this study were PTSD as the dependent variable, and coping skills, emotional intelligence, attachment, and resiliency as the four independent variables. The tables describing each of these variables in terms of their conceptual, instrumental, and operational definitions, as well as a description of the identified instruments that were utilized to evaluate for each variable may be found in the appendices under Appendix D. 


\section{Instruments}

\section{Post-Traumatic Stress Disorder}

The dependent variable in this study was PTSD. The instrument that was used to identify respondent match with PTSD symptoms was the PTSD Checklist, which is also known as the PCL-5. It was created to evaluate the presence and severity of PTSD symptoms as defined in the DSM-5. It is a 20-item self-report measure using a five-point Likert scale $(0=$ Not at all; $1=A$ little bit; 2 = Moderately; 3 = Quite a bit; 4 = Extremely). Three formats of the PCL-5 are available; one that includes Criterion A items, one that does not include Criterion A items, and one with both Criterion A items and Life Events Checklist for DSM-5 (LEC-5). The format used for this study was the PCL-5 without the Criterion A items or the LEC-5.

The PCL-5 addresses each of the criteria that is used in the DSM-5 to identify the presence of PTSD. However, the PCL-5 acts as a screener, indicating whether the participant meets the criteria for PTSD as per DSM-5 criteria, which would then require a diagnostic interview to formally diagnose the participant with PTSD. Additionally, the PCL-5 identifies each of the diagnostic criteria in a quantitative manner, whereas the DSM-5 describes the quality of the symptoms. This means that the PCL-5 addresses PTSD in more of an empirical manner, with cut-off scores indicating whether the participant has PTSD or not, as well as indicating the nature of how the participant experiences PTSD being indicated by their score; in other words, the greater the participants' score, the greater the experience and severity of PTSD. The DSM-5 identifies PTSD through the use of determining whether the individual meets the criteria as set forth in the five DSM-5 clusters. This criteria is as follows: Cluster A requires that the individual have been exposed to a traumatic event; Cluster B requires the presence of at least one of the five listed intrusion symptom; C requires the presence of one or both of the symptoms; 
Cluster D requires the presence of at least two of the seven listed symptoms; and Cluster E requires the presence of at least two of the six listed symptoms.

Symptom severity ranges were obtained by summing the scores for all 20 -items together (score range $=0-80$ ), and severity scores are obtained by summing the scores for each of the items within a given Criterion. Criterion A-E correspond directly with Clusters A-E in the DSM5. Because the terms may be used interchangeably, they were referred to as criterion, keeping in line with the terminology used in the PCL-5. Criterion items are as follows: Criterion B = items 1-5; Criterion $\mathrm{C}=$ items 6-7; Criterion $\mathrm{D}=$ items 8-14; and Criterion $\mathrm{E}=$ items 15-20. Criterion B measures intrusion symptoms; Criterion C measures avoidance symptoms; Criterion D measures negative alterations in cognitions; and Criterion E measures alterations in arousal and reactivity.

A PCL-5 cut-off score of 33 is suggested to identify that the participant meets the criteria for PTSD as set forth in the DSM-5. Specifically, the PCL-5 asks participants to rate each question by answering whether and how frequently they have experienced each particular item (if they have experienced it "a little bit;" "moderately;" "quite a bit;" or "extremely") or not (if they have experienced it in accordance with "not at all") within the past month. The PCL-5 demonstrated an overall Cronbach's alpha between .56 - .77 (Sveen, Bondjers, \& Willebrand, 2016). In recent tests examining the psychometric properties of the PCL-5 in relation to recent DSM-5 changes to PTSD criteria, Blevins, Weathers, Davis, Sittle, and Domino (2015) report that "PCL-5 scores exhibited strong internal consistency $(\alpha=.94)$, test-retest reliability $(\mathrm{r}=.82)$, and convergent ( $\mathrm{rs}=.74$ to .85$)$ and discriminant ( $\mathrm{rs}=.31$ to .60$)$ validity" (p. 489). Table 3, below, indicates the items for each of the criterion. 


\section{Table 3}

PTSD as Measured by the PCL-5

\begin{tabular}{|c|c|}
\hline \multirow{2}{*}{$\begin{array}{l}\text { Criterion and Symptom Theme } \\
\text { Criterion B: Intrusion }\end{array}$} & Item \\
\hline & $\begin{array}{l}\text { Repeated, disturbing, and unwanted memories of the stressful experience? } \\
\text { Repeated, disturbing dreams of a stressful experience? } \\
\text { Suddenly feeling or acting as if the stressful experience were actually } \\
\text { happening again (as if you were actually back there reliving it)? } \\
\text { Feeling very upset when something reminded you of a stressful } \\
\text { experience? } \\
\text { Having strong physical reactions when something reminded you of the } \\
\text { stressful experience (for example, heart pounding, trouble breathing, } \\
\text { sweating)? }\end{array}$ \\
\hline Criterion C: Avoidance & $\begin{array}{l}\text { Avoiding memories, thoughts, or feelings related to the stressful } \\
\text { experience? } \\
\text { Avoiding external reminders of the stressful experience (for example, } \\
\text { people, places, conversations, activities, objects, or situations)? }\end{array}$ \\
\hline $\begin{array}{l}\text { Criterion D: Negative Alterations } \\
\text { in Cognitions }\end{array}$ & $\begin{array}{l}\text { Trouble remembering important parts of the stressful experience? } \\
\text { Having strong negative beliefs about yourself, other people, or the world } \\
\text { (for example, having thoughts such as: I am bad, there is something } \\
\text { seriously wrong with me, no one can be trusted, the world is completely } \\
\text { dangerous)? } \\
\text { Blaming yourself or someone else for the stressful experience or what } \\
\text { happened after it? } \\
\text { Having strong negative feelings such as fear, horror, anger, guilt, or } \\
\text { shame? } \\
\text { Loss of interest in activities that you used to enjoy? } \\
\text { Feeling distant or cut off from other people? } \\
\text { Trouble experiencing positive feelings (for example, being unable to feel } \\
\text { happiness or have loving feelings for people close to you)? }\end{array}$ \\
\hline $\begin{array}{l}\text { Criterion E: Alterations in Arousal } \\
\text { and Reactivity }\end{array}$ & $\begin{array}{l}\text { Irritable behavior, angry outbursts, or acting aggressively? } \\
\text { Taking too many risks or doing things that could cause you harm? } \\
\text { Being "superalert" or watchful or on guard? } \\
\text { Feeling jumpy or easily startled? } \\
\text { Having difficulty concentrating? } \\
\text { Trouble falling or staying asleep? }\end{array}$ \\
\hline
\end{tabular}

\section{Coping}

The first independent variable in this study, Coping, is described by Carver and Scheier

(1994) as the manner in which people react to and cope with stressors and difficulties in their

lives, and the tools that they utilize in response or reaction to those stressors and while under

stress. The Brief COPE is a shortened version of the COPE, and was created by identifying 
strong factor loadings on the COPE. The results of factor analysis revealed 14 coping responses: Self-distraction, Active coping, Denial, Substance use, Use of emotional support, Use of instrumental support, Behavioral disengagement, Venting, Positive reframing, Planning, Humor, Acceptance, Religion, and Self-blame that fall into the four subscales Use of Emotional Social Support; Use of Problem Solving; Avoidance Coping, and Use of Positive Thinking.

The Brief Cope consists of 28 -items, with two items per subscale, which respondents answer as a self-report questionnaire. Each coping strategy asks about two items that represent the individual's way of coping using either their thoughts or their actions when under stress or when in difficult situations. Respondents answer based on a four-point Likert scale ( 1 = I haven't been doing this at all; 2 = I've been doing this a little bit; $3=$ I've been doing this a medium amount; 4 = I've been doing this a lot). Table 4, below, indicates the items that make up each of the 14 coping styles for this measure. 
Table 4

Brief COPE Coping Strategies

\begin{tabular}{|c|c|}
\hline Coping Strategy & Items \\
\hline Self-Distraction & $\begin{array}{l}\text { I've been turning to work or other activities to take my mind off things. } \\
\text { I've been doing something to think about it less, such as going to movies, watching } \\
\text { TV, reading, daydreaming, sleeping, or shopping. }\end{array}$ \\
\hline Active Coping & $\begin{array}{l}\text { I've been concentrating my efforts on doing something about the situation I'm in. } \\
\text { I've been taking action to try to make the situation better. }\end{array}$ \\
\hline Denial & $\begin{array}{l}\text { I've been saying to myself "this isn't real." } \\
\text { I've been refusing to believe that it has happened. }\end{array}$ \\
\hline Substance Use & $\begin{array}{l}\text { I've been using alcohol or other drugs to make myself feel better. } \\
\text { I've been using alcohol or other drugs to help me get through it. }\end{array}$ \\
\hline $\begin{array}{l}\text { Use of Emotional } \\
\text { Social Support }\end{array}$ & I've been getting emotional support from others. \\
\hline $\begin{array}{l}\text { Use of Instrumental } \\
\text { Social Support }\end{array}$ & $\begin{array}{l}\text { I've been getting comfort and understanding from someone. } \\
\text { I've been getting help and advice from other people. } \\
\text { I've been trying to get advice or help from other people about what to do. }\end{array}$ \\
\hline $\begin{array}{l}\text { Behavioral } \\
\text { Disengagement }\end{array}$ & $\begin{array}{l}\text { I've been giving up trying to deal with it. } \\
\text { I've been giving up the attempt to cope }\end{array}$ \\
\hline Venting & $\begin{array}{l}\text { I've been saying things to let my unpleasant feelings escape. } \\
\text { I've been expressing my negative feelings. }\end{array}$ \\
\hline Positive Reframing & $\begin{array}{l}\text { I've been trying to see it in a different light, to make it seem more positive. } \\
\text { I've been looking for something good in what is happening. }\end{array}$ \\
\hline Planning & $\begin{array}{l}\text { I've been trying to come up with a strategy about what to do. } \\
\text { I've been thinking hard about what steps to take. }\end{array}$ \\
\hline Humor & $\begin{array}{l}\text { I've been making jokes about it. } \\
\text { I've been making fun of the situation. }\end{array}$ \\
\hline Acceptance & $\begin{array}{l}\text { I've been accepting the reality of the fact that it has happened. } \\
\text { I've been learning to live with it. }\end{array}$ \\
\hline Religion & $\begin{array}{l}\text { I've been trying to find comfort in my religion or spiritual beliefs. } \\
\text { I've been praying or meditating. }\end{array}$ \\
\hline Self-Blame & $\begin{array}{l}\text { I've been criticizing myself. } \\
\text { I've been blaming myself for things that happened. }\end{array}$ \\
\hline
\end{tabular}

For this variable, the 14 subscales of the COPE were evaluated using the four factors as identified by Gill (2005) in his study on coping style as a predictor in PTSD. This study appeared to match most closely the variables examined in this study; therefore, it appeared to be the most beneficial manner in which to examine coping for the study at hand. The four identified 
coping skills were as follows: Use of Emotional Social Support; Use of Problem Solving;

Avoidance Coping, and Use of Positive Thinking. Endorsement items for each factor is shown

below in Table 5 .

Table 5

Brief COPE Subscales

\begin{tabular}{|c|c|c|}
\hline Subscale & Coping Style & Items \\
\hline \multirow{6}{*}{$\begin{array}{l}\text { Use of } \\
\text { Emotional } \\
\text { Social } \\
\text { Support }\end{array}$} & Use of Emotional Social Support & I've been getting emotional support from others. \\
\hline & & I've been getting comfort and understanding from someone. \\
\hline & Venting & I've been saying things to let my unpleasant feelings escape. \\
\hline & & I've been expressing my negative feelings. \\
\hline & Use of Instrumental Social Support & I've been getting help and advice from other people. \\
\hline & Religion & $\begin{array}{l}\text { I've been trying to get advice or help from other people about what to do. } \\
\text { I've been trying to find comfort in my religion or spiritual beliefs. } \\
\text { I've been praying or meditating. }\end{array}$ \\
\hline \multirow{3}{*}{$\begin{array}{l}\text { Use of } \\
\text { Problem } \\
\text { Solving }\end{array}$} & Active Coping & $\begin{array}{l}\text { I've been concentrating my efforts on doing something about the situation } \\
\text { I'm in. }\end{array}$ \\
\hline & & I've been taking action to try to make the situation better. \\
\hline & Planning & $\begin{array}{l}\text { I've been trying to come up with a strategy about what to do. } \\
\text { I've been thinking hard about what steps to take. }\end{array}$ \\
\hline \multirow{8}{*}{$\begin{array}{l}\text { Avoidance } \\
\text { Coping }\end{array}$} & Self-Distraction & I've been turning to work or other activities to take my mind off things. \\
\hline & & $\begin{array}{l}\text { I've been doing something to think about it less, such as going to movies, } \\
\text { watching TV, reading, daydreaming, sleeping, or shopping. }\end{array}$ \\
\hline & Denial & I've been saying to myself "this isn't real." \\
\hline & & I've been refusing to believe that it has happened. \\
\hline & Substance Use & $\begin{array}{l}\text { I've been using alcohol or other drugs to make myself feel better. } \\
\text { I've been using alcohol or other drugs to help me get through it. }\end{array}$ \\
\hline & Behavioral Disengagement & I've been giving up trying to deal with it. \\
\hline & Self-Blame & $\begin{array}{l}\text { IVe been giving up the attempt to cope } \\
\text { I've been criticizing myself. }\end{array}$ \\
\hline & & I've been blaming myself for things that happened. \\
\hline \multirow{4}{*}{$\begin{array}{l}\text { Use of } \\
\text { Positive } \\
\text { Thinking }\end{array}$} & Positive Reframing & $\begin{array}{l}\text { I've been trying to see it in a different light, to make it seem more positive. } \\
\text { I've been looking for something good in what is happening. }\end{array}$ \\
\hline & Humor & I've been making jokes about it. \\
\hline & & I've been making fun of the situation. \\
\hline & Acceptance & $\begin{array}{l}\text { I've been accepting the reality of the fact that it has happened. } \\
\text { I've been learning to live with it. }\end{array}$ \\
\hline
\end{tabular}

Higher scores indicate specific coping styles. Total scores on each of the scales are calculated by summing the appropriate items for each scale. No items are reverse scored. There is no overall total score, only total scores for each of the scales. Specifically, the Brief COPE asks participants to rate each statement by expressing how much or how frequently they have 
been using a particular coping strategy (if they have been "doing it a little, a medium amount, or a lot") or not (if they have not "been doing it at all"); answering all items and not leaving any items blank; being as accurate as possible and keeping each of their answers separate from the others in their mind, and not answering based on whether the coping strategies seem to be working for them or not. Cronbach's alpha ranges from .72 to .84 on this measure, with reliability being the highest for Planning (Chronbach's $\alpha=.73$ ), Humor (Chronbach's $\alpha=.73$ ), Religion (Chronbach's $\alpha=.82$ ), Emotional Support (Chronbach's $\alpha=.71$ ), Self-Distraction (Chronbach's $\alpha=.71$ ), and Substance Use (Chronbach's $\alpha=.90$ ). Table 6, below, shows the validity and reliability for each of the 14 subscales of the Brief COPE:

\section{Table 6}

Validity and Reliability of the Brief COPE

\begin{tabular}{llll}
\hline Brief COPE: & Test-retest (ICC) & Internal Consistency & Discriminant Validity \\
\hline Active coping & & & \\
Planning & 0.42 & 0.83 & $p<0.01$ \\
Positive reframing & $<0.00$ & 0.60 & NS \\
Acceptance & 0.99 & 0.80 & $p<0.05$ \\
Humor & 0.32 & 0.81 & NS \\
Religion & 0.45 & 0.92 & NS \\
Using emotional support & 0.33 & 0.72 & NS \\
Using instrumental support & 0.36 & 0.83 & NS \\
Self-distraction & 0.50 & 0.57 & NS \\
Denial & 0.97 & 0.58 & NS \\
Venting & 0.92 & 0.54 & NS \\
Substance use & 0.03 & 1.00 & NS \\
Behavioral disengagement & 1.00 & 0.74 & NS \\
Self-blame & 0.94 & 0.25 & \\
\hline Yust, Low, $\&$ Yip, 2009, p. 41 & & & \\
\hline
\end{tabular}

Yusoff, Low, \& Yip, 2009, p. 41

\section{Emotional intelligence}

The second independent variable, Emotional intelligence, has been defined by Mayer and Salovey (1997) as the ability to understand emotion, being able to utilize one's own emotion to assist in facilitating the thinking process, and being able to manage both one's own emotion and others' emotions, and includes the perception and expression of emotion. The Schutte 
Emotional Intelligence Scale (SEIS), which is also known as the Schutte Self-Report Emotional Intelligence Test (SSEIT) and the Self-Report Emotional Intelligence Test (SREIT), is a 33-item self-report measure that uses the emotional intelligence model as proposed by Salovey and Mayer (Salovey \& Mayer, 1990; Salovey, Mayer, Goldman, Turvey, \& Palfai, 1995) as its theoretical guide, with the respondent's score falling into one of three ranges: Unusually Low, Normative/Average, or Unusually High.

However, the theoretical guide proposed by Schutte, Malouff, and Bhullar (2009) consists of an individual's ability to both understand and to regulate their own emotions, as well as being able to understand and regulate emotions in others. Although Salovey and Mayer (1990) proposed the three aspects of emotional intelligence as consisting of "how one appraises and expresses emotion, the individual's ability to regulate emotion, and how emotion is utilized in the individual's life" (Consortium for Research on Emotional Intelligence in Organizations, 2017, p. 160), the model created by Schutte, Malouff, and Bhullar "proposed that emotional intelligence consists of appraisal of emotion in the self and others, expression of emotion, regulation of emotion in the self and others, and utilization of emotion in solving problems (Schutte, Malouff. \& Bhullar, 2009, p. 119). Along with others (Petrides \& Furnham, 2000; Saklofske, Austin, \& Minski. 2003), Ciarrochi, Deane, and Anderson (2002) performed factor analytic studies, which resulted in four subscales of emotional intelligence: Managing Own Emotions; Managing Others' Emotions; Perception of Emotion; and Utilization of Emotion. Table 7 shows the breakdown of items contained in each of these four subscales: 
Table 7

Emotional Intelligence Subscales

\begin{tabular}{|c|c|}
\hline Subscale & Item \\
\hline Perception of Emotion & $\begin{array}{l}\text { I find it hard to understand the non-verbal messages of other people } \\
\text { I am aware of my emotions as I experience them. } \\
\text { I am aware of the non-verbal messages I send to others. } \\
\text { By looking at their facial expressions, I recognize the emotions people are experiencing. } \\
\text { I know why my emotions change. } \\
\text { I easily recognize my emotions as I experience them. } \\
\text { I am aware of the non-verbal messages other people send. } \\
\text { I know what other people are feeling just by looking at them. } \\
\text { I can tell how people are feeling by listening to the tone of their voice. } \\
\text { It is difficult for me to understand why people feel the way they do. }\end{array}$ \\
\hline $\begin{array}{l}\text { Managing Own } \\
\text { Emotions }\end{array}$ & $\begin{array}{l}\text { When I am faced with obstacles, I remember times I faced similar obstacles and } \\
\text { overcame them. } \\
\text { I expect that I will do well on most things I try. } \\
\text { I expect good things to happen. } \\
\text { When I experience a positive emotion, I know how to make it last. } \\
\text { I seek out activities that make me happy. } \\
\text { I have control over my emotions. } \\
\text { I motivate myself by imagining a good outcome to tasks I take on. } \\
\text { When I am faced with a challenge, I give up because I believe I will fail } \\
\text { I use good moods to help myself keep trying in the face of obstacles. }\end{array}$ \\
\hline Managing Others' & I know when to speak about my personal problems to others. \\
\hline Emotions & $\begin{array}{l}\text { Other people find it easy to confide in me. } \\
\text { I like to share my emotions with others. } \\
\text { I arrange events others enjoy. } \\
\text { I present myself in a way that makes a good impression on others. } \\
\text { I compliment others when they have done something well. } \\
\text { When another person tells me about an important event in his or her life, I almost feel } \\
\text { as though I experienced this event myself. } \\
\text { I help other people feel better when they are down }\end{array}$ \\
\hline Utilization of Emotion & $\begin{array}{l}\text { Some of the major events of my life have led me to re-evaluate what is important and } \\
\text { not important. } \\
\text { When my mood changes, I see new possibilities. } \\
\text { Emotions are one of the things that make my life worth living. } \\
\text { When I am in a positive mood, solving problems is easy for me. } \\
\text { When I am in a positive mood, I am able to come up with new ideas. } \\
\text { When I feel a change in emotions, I tend to come up with new ideas. }\end{array}$ \\
\hline
\end{tabular}

Respondents answer based on a five-point Likert scale $(1=$ strongly disagree; $2=$

somewhat disagree; $3=$ neither agree nor disagree; $4=$ somewhat agree; $5=$ strongly agree).

Specifically, the SEIS asks participants to rate each statement by expressing how much they

agree with it (if they do generally agree) or how much they disagree with it (if they generally

disagree). The mean score is 124 , with a standard deviation of 13 . Scores below 111 or above 
137 are considered to fall into an "unusually low" or "unusually high" range (Schuette, Malouff, Hall, Haggerty, Cooper, Golden, \& Dornheim, 1998).

The SEIS has shown to demonstrate Cronbach's alpha internal consistency ratings of 0.90, and a two-week test-retest reliability of 0.78 (Schuette, Malouff, Hall, Haggerty, Cooper, Golden, \& Dornheim, 1998). Validity has been addressed in multiple studies, but results have been varied, with reports that some researchers have used measures of pathology as a means of validating the coping scales (Donoghue, 2004; Stone et al., 1992), while other researchers report that distress has been a factor in contaminating the outcome of attempts to validate coping measures (Donoghue, 2004; Connor-Smith et al., 2000; Compas et al., 2001). There has also been difficulty in comparisons of coping measures due to each measuring a different aspect of coping (Donoghue, 2004).

\section{Attachment}

The third independent variable, Attachment, was defined by Bowlby $(1973,1982)$, who proposed attachment to be the style of behavior by which humans connect with other human beings (Mikulincer, Solomon, Shaver, and Ein-Dor, 2014). The Measure of Attachment Qualities (MAQ) was created by Charles S. Carver (1997a), and measures four subscales of attachment: avoidant attachment, anxious attachment, ambivalent attachment, and secure/insecure attachment. It uses a 14-item self-report measure in which respondents are provided with a four-point Likert scale ( 1 = I DISagree with the statement a lot; 2 = I DISagree with the statement a little; 3 = I agree with the statement a little; $4=\mathrm{I}$ agree with the statement a lot). Higher scores indicate specific attachment styles. Total scores on each of the scales are calculated by summing the appropriate items for each scale. Items 4,8 , and 9 are reverse scored. 
There is no overall total score, only total scores for each of the scales. Table 8 , below, indicates which items are associated with which subscales.

\section{Table 8}

MAQ Subscale Items

\begin{tabular}{|c|c|}
\hline Subscale & Item \\
\hline Avoidance Attachment & $\begin{array}{l}\text { I get uncomfortable when someone wants to be very close. } \\
\text { I find it easy to be close to others. } \\
\text { I prefer not to be too close to others. } \\
\text { I am very comfortable being close to others. } \\
\text { Others want me to be more intimate than I feel comfortable being. }\end{array}$ \\
\hline $\begin{array}{l}\text { Ambivalence-Worry } \\
\text { (Ambivalent) Attachment }\end{array}$ & $\begin{array}{l}\text { I often worry that my partner doesn't really love me. } \\
\text { I often worry my partner will not want to stay with me. } \\
\text { I don't worry about others abandoning me. }\end{array}$ \\
\hline $\begin{array}{l}\text { Ambivalence-Merger } \\
\text { (Anxious) Attachment }\end{array}$ & $\begin{array}{l}\text { I have trouble getting others to be as close as I want them to be. } \\
\text { I find others are reluctant to get as close as I would like. } \\
\text { My desire to merge sometimes scares people away. }\end{array}$ \\
\hline Secure Attachment & $\begin{array}{l}\text { When I'm close to someone, it gives me a sense of comfort about life in general. } \\
\text { It feels relaxing and good to be close to someone. } \\
\text { Being close to someone gives me a source of strength for other activities. }\end{array}$ \\
\hline
\end{tabular}

Specifically, the MAQ asks participants to rate each statement by expressing how much they agree with it (if they do generally agree) or how much they disagree with it (if they generally disagree); answering all items and not leaving any items blank; being as accurate as possible, and not allowing their answers to any one item influence their answer to any of the other items ("treat each one as though it is completely unrelated to the others;" and responding based on their own personal feelings and opinions. Cronbach's alpha ranged from .69 to .76 on this measure, with reliability being the highest for Avoidance (Chronbach's $\alpha=.76$ ), followed by Ambivalence-Merger (Chronbach's $\alpha=.73$ ), Security (Chronbach's $\alpha=.72$ ), and AmbivalenceWorry (Chronbach's $\alpha=.69)$. Validity is shown in Table 9 below: 
Table 9

Measure of Attachment Qualities (MAQ) Validity

\begin{tabular}{|c|c|c|c|c|}
\hline Item and A Priori Designation & Avoidant & Ambivalent & Anxious & Secure \\
\hline \multicolumn{5}{|l|}{ Avoidance $(\alpha=.76, M=2.02, S D=.68$, test-retest $r=.80)$} \\
\hline I get uncomfortable when someone wants to be very close $(\mathrm{Av})$ & 0.76 & & & \\
\hline I find it easy to be close to others (S) & -0.72 & & & \\
\hline I prefer not to be close to others (Av) & 0.72 & & & \\
\hline I am very comfortable being close to others $(\mathrm{S})$ & -0.70 & & & \\
\hline Others want me to be more intimate than I feel comfortable being (Av) & 0.65 & & & \\
\hline \multicolumn{5}{|l|}{ Ambivalence-Worry ( $\alpha=.69, M=2.14, S D=.79$, test-retest $r=.69$ ) } \\
\hline I often worry that my partner doesn't really love me (Amb) & & 0.88 & & \\
\hline I often worry that my partner will not want to stay with me (Amb) & & 0.88 & & \\
\hline I don't worry about others abandoning me (S) & & -0.54 & & \\
\hline \multicolumn{5}{|l|}{ Ambivalence-Merger $(\alpha=.73, M=1.89, S D=.70$, test-retest $\mathrm{r}=.69)$} \\
\hline I have trouble getting others to be as close as I would like (Amb) & & & 0.80 & \\
\hline I find others often are reluctant to get as close as I would like (Amb) & & & 0.79 & \\
\hline My desire to merge sometimes scares people away (Amb) & & & 0.79 & \\
\hline \multicolumn{5}{|l|}{ Security $(\alpha=.72, M=3.45, S D=.59$, test-retest $r=.61)$} \\
\hline \multicolumn{3}{|c|}{ When I am close to someone it gives me a sense of comfort about life in general (S) } & & 0.79 \\
\hline \multicolumn{2}{|l|}{ It feels relaxing and good to be close to someone (S) } & & & 0.79 \\
\hline Being close to someone gives me a source of strength for other activities & & & & 0.77 \\
\hline
\end{tabular}

Carver, 1997a, p. 868

\section{Resilience}

The final independent variable, Resilience, is defined by Connor and Davidson (2003) as "the personal qualities that enable one to thrive in the face of adversity" (p. 76). The Connor Davidson - Resilience Scale was developed because the researchers felt that there was a need for "a stronger presence in the field of PTSD assessment" among the measures that were available, and that despite the fact that resiliency measures existed, "they had largely failed to penetrate into the world of clinical practice" (http://www.connordavidson-resiliencescale.com/about.php). Therefore, the CD-RISC was developed to meet this need. There are three versions of the Connor-Davidson - Resilience Scale (CD-RISC). There is a 25-item CD-RISC (CD-RISC 25), and two briefer versions; the 10 item (CD-RISC 10) and the two item (CD-RISC 2) scales 
(http://www.connordavidson-resiliencescale.com/about.php). The CD-RISC 10 was the measure used for this study. The authors propose the following with regard to the 1 - item version of the scale:

The 10 item version (score range 0-40) comprises items 1, 4, 6, 7, 8, 11, 14, 16, 17, 19 from the original scale, and was developed by Drs. Campbell-Sills and Stein, at the University of California, San Diego, on the basis of factor analysis. In a community survey of 764 US adults, a mean score of $31.8(\mathrm{SD}=5.4)$ was obtained for the CD-RISC 10 (Campbell-Sills L, Forde DR, Stein MB. J Psychiatric Research (2009), doi:10.1016/j.jpsychires.2009.01.013). (http://www.connordavidsonresiliencescale.com/about.php)

The Connor Davidson - Resiliency Scale (CD-RISC) was created by Kathryn M. Connor and Jonathan R.T. Davidson as a means to measure resiliency. It uses a 10-item self-report measure in which respondents are provided with a 5-point Likert scale ranging from $0-4(0=$ never; 1 = rarely true; $2=$ sometimes true; $3=$ often true; and $4=$ true nearly all of the time). Total scores on each of the scales are calculated by summing the appropriate items for each scale (CD-RISC, CD-RISC 25, CD-RISC 10, or the CD-RISC 2).

Specifically, the CD-RISC asks participants to rate each statement by expressing how much they disagree with it (if it is "not true at all") or how much they agree with it (if it is "true nearly all the time") as applicable to them in the previous month. The items are totaled to yield a global scale score ranging from a minimum of 0 to a maximum of 40 that indicates the respondent's level of resilience. Higher scores indicate a greater level of resilience. Written permission to explore the psychometric properties of the scale was obtained from the authors. Davidson and Connor (2018) report the following:

Quartiles describe four groups of equal number taken from the observed distribution of scores, with the first quartile (Q1) describing the score range for the lowest group (lowest $25 \%$ of the population), i.e. the least resilient, the second (Q2) and third (Q3) the 
intermediate scores, and the fourth $(\mathrm{Q} 4)$ describing the highest or most resilient, i.e. above $75 \%$ of the population. For the CD-RISC-10 $(n=764)$, median score was 32 , with lowest to highest quartiles being 0-29, 30-32, 33-36 and 37-40 (Campbell-Sills, Forde, \& Stein, 2009 as cited in Davidson \& Connor, 2018), indicating the following quartile interpretations: $0-29=$ Lowest $25 \%$ of the population; $30-32=$ Second lowest quartile of the population; $33-36=$ Third quartile of the population; and $37-40=$ Highest $25 \%$ of the population (Davidson \& Connor, 2018, p. 6). Similar results were obtained in another US population $(n=577)$ by Davidson and Lee. A score of 26 would fall in the lowest $25 \%$ of the population, a score of 36 would be the third quartile and $25 \%$ of the population would score higher (i.e. 37-40). (p. 6). Table 10, below, shows the breakdown of the scoring for the CD-RISC:

\section{Table 10}

CD-RISC Scoring Breakdown

\begin{tabular}{lll}
\hline Scoring Range & Quartile & Interpretation \\
\hline $0-29$ & Lowest 25\% of the population & The least resilient of the population \\
$30-32$ & Second lowest quartile of the population & The second least resilient of the population \\
$33-36$ & Third quartile of the population & The second most resilient of the population \\
$37-40$ & Highest 25\% of the population & The highest or most resilient of the population \\
\hline
\end{tabular}

Studies on the CD-RISC have demonstrated internal consistencies with Cronbach's alpha's between 0.81 to 0.87 (Jung et al, 2017; Baek et al, 2010; Yu and Zhang, 2007). Other studies by Ito et al (2009) have demonstrated internal consistencies of Cronbach's $\alpha=0.94$ and 0.90 for two samples, and good test-retest reliability of 0.94 and 0.83 . Convergent validity has been shown "relative to measures of depression ( $r=0.51$ both scales), self-efficacy $(r=0.47$ and 0.45 for the 25 and 10 item scales), self-mastery $(r=0.29$ and $r=0.31)$ and social support $(r$ 
$=0.27$ and $r=0.21$ ), and divergent validity has been shown relative to handgrip strength and body mass index (non-significant or weakly significant correlations)" (Knows-His-Gun et al, 2013, as cited in Davidson \& Connor, 2018, p. 51). According to the creators of the CD-RISC, “construct validity comes from a study by Roberts et al. (2007), which showed that among 252 veterans of Iraq or Afghanistan, those with higher resilience were less likely to develop PTSD $(\mathrm{OR}=0.11,95 \% \mathrm{CI}=0.06-0.21)$, and of those who did have PTSD, resiliency was uniquely associated with decreased PTSD severity after accounting for demographic variables and combat severity $(\beta=-.0 .37, \mathrm{p}<0.001)$ )" (p. 53).

\section{Procedure}

\section{Data Collection}

Prior to the initiation of the research, a formal application was submitted to the Institutional Review Board (IRB) of Andrews University. The Institutional Review Board consists of a designated group of individuals who review and monitor research that involves human participants. The purpose of the IRB is to protect the rights and welfare of individuals who choose to participate in research studies, and the IRB committee has the authority to approve or disapprove research studies, or to require that researchers modify their studies in accordance with guidelines for research. Once a research study is approved, the IRB then continues to monitor the ongoing progress of the approved studies, and approves any modifications that may come up, taking action to protect research participants should the need arise, including suspending research projects that the IRB anticipates may go outside of the guidelines and boundaries that are set for the protection of participants. The IRB reviewed the application and supporting materials, asking questions to define and refine the specific research process, after which the application was approved (IRB Protocol approval number19-059). 
Following this, a total of 300 Emergency Medical Technicians (EMTs) from the United States were sought to be recruited for participation in the completion of study materials. Participants were selected based on the following eligibility criteria: Eligible participants were identified as being: 1) be an individual who works in the Emergency Medical Services field as a private sector EMT; 2) a private sector field EMT who has been exposed to traumatic events as a result of their job description and working conditions; or 3) an individual who has worked in the private sector as an EMT within the previous 5 years and who had been exposed to traumatic events as a result of their job description and working conditions. Exclusion criteria included 1) individuals who are first responders, but who have never worked in the private sector of the EMS field; 2) individuals who are first responders and have worked in the private sector of the EMS field, but not within the previous 5 years or who are working in the public sector currently; and 3) individuals who are first responders, but do not currently, nor have they ever, worked in the private sector.

Individuals were selected through the use of research advertisements that were sent out through social media sites, such as Facebook, through the Michigan State DHHS, by placement on friend's social media sites, and by word of mouth. Although the sample size for this study was estimated through power analysis with a total estimated sample size of 300 participants, out of a total of 113 respondents, only 50 were eligible to proceed with and complete the survey instruments for the quantitative portion of the study. From this sample, participants were selected for inclusion in the study through the full completion of all survey instruments. Additionally, due to the limited number of eligible participants resulting in the quantitative portion of the study, participants were given the option of participating in the qualitative portion of the study, with only three participants responding to the invitation for additional participation. 
Each of these three respondents participated fully in the qualitative portion of the study following their completion of the quantitative survey instruments.

This researcher served as the principal investigator in the collection of data, along with the Chairperson for this dissertation, Dr. Dennis Waite, and the Doctoral Committee Members, Drs Jimmy Kijai and Harvey Burnett. Participants all completed a pre-screening questionnaire that consisted of demographic information regarding age, gender, gender identity, cultural background, field status (whether private or public sector employee, and scope of their practice (whether an EMT-B, EMT-I, or an EMT-P) (See Appendix C).

Participants were provided with a link that enabled them to begin the survey process. However, prior to responding to survey instrument questions, participants were provided with information about the survey, along with any potential risks and benefits. Participants were informed that they had the right to discontinue the survey at any point in the research process without question or repercussion, and were provided with a full copy of the informed consent (see Appendix B) to read. Included in this section was information outlining the terms of the study, the length of the measures that were used, the inclusion and exclusion criteria, and on the use and destruction of both the research materials and all confidential information obtained as part of the research process. Participants indicated their acknowledgement, understanding, and consent by clicking on either a "yes" or a "no" button at the end of the section. Participants clicking on the "no" button were not permitted to continue with the research process. Participants clicking on the "yes" button were permitted to continue, at which time screening questions were presented to ensure that participants met the eligibility criteria for the study. Additional screening information included two questions asking whether the participant was currently working in the public sector of the field, and whether the participant was currently, or 
had within the previous five years, worked in the private sector of the field. Following the provision of their consent for the study, each participant continued forward to complete the intake/information survey and each measure, all of which were provided in an online-survey style format. No personally identifiable information (PII), such as participant names, was obtained from participants.

All study data was retrieved from SurveyMonkey, and entered into SPSS by SurveyMonkey and sent to this researcher. Web survey tools used that host the survey in-house, was secured on a fire-walled, password-protected server. Access to survey and research information was open to all participants, as listed above. Because the total number of participants were unable to be obtained, and additional participants were no longer responding to the advertisements for participants, nor were the links were no longer generating any hits, the research was closed with 50 eligible participants being obtained for the quantitative portion of the study. All information gathered for this study was kept anonymous and confidential.

At the conclusion of the quantitative survey portion of the study, each participant was provided the option of participating in the qualitative portion of the study, with only four participants choosing that option. Of these four individuals, only three were eligible for the study. As with the quantitative portion of the study, this researcher served as the investigator in the actual collection of the data, along with the Chairperson for this study, Dr. Dennis Waite, and the Doctoral Committee Members, Drs Jimmy Kijai and Harvey Burnett. Because participants had all completed the consent form, they were not required to provide additional consent for the qualitative portion of the study; however, to ensure eligibility, all four participants were again asked the same pre-screening questions, as contained in the quantitative portion of the study. These again were as follows: Eligible participants were identified as being: 1) be an individual 
who works in the Emergency Medical Services field as a private sector EMT; 2) a private sector field EMT who has been exposed to traumatic events as a result of their job description and working conditions; or 3) an individual who has worked in the private sector as an EMT within the previous 5 years, and who had been exposed to traumatic events as a result of their job description and working conditions. Exclusion criteria included 1) individuals who are first responders, but who have never worked in the private sector of the EMS field; 2) individuals who are first responders and have worked in the private sector of the EMS field, but not within the previous 5 years or who are working in the private sector currently; and 3) individuals who are first responders, but do not currently, nor have they ever, worked in the private sector.

Participants were informed of the terms and lengths of the interview, and verbal consent to be audiotaped consent form, and were reminded of the confidentiality, use and destruction of both the research materials and confidential information materials, and were emailed an outline of the questions inherent in that portion of the study. Eligible participants continued on with the interview questions. Each of the qualitative interviews were set for a total of two-hours in length, with verbal consent for additional time to be used should the necessity arise. Each of the interviews were audio taped, with the dialogue being transcribed digitally for the use of analysis. All information obtained for this study was kept anonymous and confidential, and all interviewees participated on a voluntary basis.

\section{Qualitative data}

Following the transcription of each of the participant interviews, the interviews were then evaluated through the use of open, line-by-line coding. Following the coding of the interviews, the interviews were axial coded in order to identify for the presence of common themes among the three participants. In addition to coding each of the interviews, this researcher performed 
memo writing on the transcripts for further evaluation, and to identify any additional themes that may have been missed previously. Following the identification of categories from the transcripts, codes were identified in order to identify any themes that were specific to each of the participants themselves, but which may not have fit into a pattern across the three participants. With one of the goals being to identify both shared and individual experiences among the participants, single participant categories were retained that had been identified among individual participants. These were included in the final analysis of the interviews.

Overlapping categories were then identified among all three of the participants, with these codes collapsed in order to identify the most common areas and themes amongst the participants as a whole. This researcher continued to return to the data throughout the analysis process in order to ensure soundness between the data as it was identified and each of the research questions. Through the utilization of this method, the validity of the qualitative portion of the study was ensured. Finally, this researcher then revisited the memos that had been written previously during the coding of the data in order to 1) identify the themes and commonalities among the participants; 2) to assist in the connection of the categories to each other; and 3) to identify the uniqueness's of each of the participants. The purpose behind this was to identify any differing populations that could be an additional area of focus in future studies on private sector EMTs, as well as to identify service needs as specific to this population, and to identify service needs of any additional but similar populations not shared by these particular participants.

\section{Data Cleaning and Preparation}

\section{Quantitative data}

Prior to beginning analysis, the data was examined to ensure that no data errors existed. This took place through an examination of the data using the Frequencies procedure with SPSS 
to obtain both descriptive statistics and frequency distributions. All quantitative data was examined to identify values that were outside the range of possibilities, with an assessment of the means and standard deviations for the data. Categorical data was evaluated for appropriateness to the parameters prescribed for each variable to ensure that all data had been entered correctly into the database; making sure that variables had been coded in correspondence to the coded values for the possible categories. Additionally, the data was evaluated to identify missing data, and to identify the most appropriate manner to deal with any missing values, as well as to look for possible patterns within the missing data, with dichotomized values $(0=$ not complete, $1=$ complete) used as the means by which missing data was assessed. Frequency counts were used to evaluate missing responses for each of the constructs. To enable for the analysis of the data, missing data was represented through the use of mean replacement, as utilizing this would produce less bias in the results less than would be present through respondent attrition. There were a total of 17 missing values for the 10 Resiliency questions.

Data was also assessed for the presence of outliers, which was done through the use of examining the frequency distributions, histograms, and boxplots of the variables. To ensure accuracy of assessment, the data was transformed to $z$-scores, with any $z$ value that was greater than \pm 3.00 indicating an outlier. With the intent being to use multivariate analysis for this research, Mahalanobis distance was employed, which was evaluated as a chi-square $\left(X^{2}\right)$ statistic with the degrees of freedom equal to the number of variables in the analysis. Any variable with a significance value $p<.001$ was examined further to determine whether it is an outlier due to data entry error, in which the data was corrected and then the data reanalyzed. Any values showing significance due to instrumentation error or due to being determined to be different from the rest of the sample were dropped from the analysis. Because outliers were viewed to 
possibly represent interesting information that could prove points of interest that would be useful for follow-up studies, it was decided to retain them if they were 2 or less standard deviations from the mean. Outliers that were greater than 3 standard deviations from the mean were considered to be extreme, and based on this, three were removed from the dataset.

Finally, assumptions of normality, linearity, and homoscedasticity were evaluated to ensure robustness of the data. Because this study used multivariate methods, normality was evaluated for using the Kolmogorov-Smirnov statistic, with Lilliefors significance level. The assumption of multivariate normality included an assessment that each of the variables were normally distributed, that any linear combination of the variables were normally distributed, and that all subsets of the variables had a normal multivariate distribution. For data that was found to violate the assumptions of normality, data transformation of the data took place, with the method of transformation identified based on the shape of the original raw data. This shape was identified through an evaluation of the skewness and kurtosis of the data. Linearity of the data was assessed for through an examination of both the residuals plots and bivariate scatterplots, and homoscedasticity was assessed statistically through an examination of Box's $M$ test for equality of variance-covariance matrices. Violations were corrected through the use of the transformation of variables.

\section{Data Analysis}

This study examined the level of PTSD symptomatology experienced by EMTs and how coping techniques, emotional intelligence, resiliency, and attachment play a role in impacting these symptoms. The data was analyzed using both SPSS and qualitative evaluation techniques, with content analysis being the primary evaluation method. To identify the unique contribution of each of the variables (coping techniques, emotional intelligence, resiliency, and attachment) in 
the explanation of PTSD symptomatology in EMTs, a Multiple Linear Regression analysis was performed. To identify specific experiences and needs of this population, individual interviews and analysis was utilized using a qualitative approach to the data.

To evaluate for and analyze the quantitative data, several analysis measures were used. To evaluate for Research Question One, the data was evaluated through the identification of the presence of PTSD as per the cutoff score on the PCL-5, as well as through the grouping of items into clusters in accordance with PCL-2 Criterion. Frequencies were then run to identify the presence of PTSD in this sample of private sector EMTs.

For Research Question Two, diagnostics were run to breakdown the EMTs in this study into their EMT status (EMT-B or EMT-P) to identify the presence of PTSD. Crosstabs were then run to identify the specific items endorsed per criterion and per EMT level. An Independent Sample T-Test was run using the sample of EMT-B's and EMT-P's to identify any differences between the scores for the two groups.

For Research Question Three, Coping was grouped into the 14 subscales of the Brief COPE to provide the reader with an understanding of which items comprised which of the subscales, after which the subscales were grouped into the four coping styles as identified by the authors. A MANOVA was run to identify the coping styles used by EMT level.

For Research Question Four, the scoring breakdown of the SEIS was shown along with the breakdown of the items comprising each of the four subscales of the measure. From there, frequencies were run to identify the levels of Emotional Intelligence for the EMTs in this study. The scoring breakdown was presented for the CD-RISC, with frequencies run to identify the levels of resiliency for the EMTs in this study. To evaluate for Attachment, the subscales were 
presented with each of the items comprising those subscales, after which frequencies were run to identify the attachment styles and their levels utilized by the private sector EMTs in this study.

For Research Question Five, a Multiple Linear Regression was run in order to identify the nature of the relationship between PTSD severity and Emotional Intelligence, Coping Skills, Resiliency, and Attachment.

\section{Summary}

In summary, a sample of 50 private sector EMTs as represented by all EMT levels (EMTBasic's - EMT-B's; EMT-Intermediates - EMT-I's; and EMT-Paramedics - EMT-P's) was evaluated for PTSD symptoms utilizing the PTSD Checklist (PCL-5), the Brief COPE, the Schuette Emotional Intelligence Scale (known as the SEIS, SSEIT, and SREIT), the Measure of Attachment Quality (MAQ), and the Brief Resilience Scale (CD-RISC) to identify how PTSD is experienced by private sector EMTs in relation to severity of PTSD, the use of coping strategies, levels of emotional intelligence, resilience, and attachment, and what the nature is, if any, of the relationship between PTSD and each of the four variables (coping skills, emotional intelligence, resiliency, and attachment). Participants were individuals who worked in the Emergency Medical Services field as a private sector EMT and who had been exposed to traumatic events as a result of their job description and working conditions, and were obtained through social media sites such as Facebook, word of mouth, and advertising through The State of Michigan Department of Health and Human Services (DHHS).

To identify specific experiences and needs of this population, individual interviews and analysis was utilized using a qualitative approach to the data. Participants also completed survey instruments for the quantitative portion of the study, with three participants choosing to also participate in the qualitative portion of the study through individual interviews. The quantitative 
data was analyzed using SPSS-26. To identify the unique contribution of each of the variables (coping techniques, emotional intelligence, resilience, and attachment) in the explanation of PTSD symptomatology in EMTs, several analysis measures were used. Univariate Analysis was used to obtain descriptive characteristics of the sample. To evaluate for Research Question One, the data was evaluated through the identification of the presence of PTSD as per the cutoff score on the PCL-5, as well as through the grouping of items into criterion in accordance with the PCL-5 Criterion. Frequencies were then run to identify the presence of PTSD in this sample of private sector EMTs. For Research Question Two, diagnostics were run to breakdown the EMTs in this study into their EMT status (EMT-B or EMT-P) to identify the presence of PTSD.

Crosstabs were then run to identify the specific items endorsed per criterion and per EMT level. An Independent Sample T-Test was run using the sample of EMT-B's and EMT-P's to identify any differences between the scores for the two groups. For Research Question Three, Coping was grouped into the 14 subscales of the Brief COPE to provide the reader with an understanding of which items comprised which of the subscales, after which the subscales were grouped into the four coping styles as identified by the authors. A MANOVA was run to identify the coping styles used by EMT level. For Research Question Four, the scoring breakdown of the SEIS was shown along with the breakdown of the items comprising each of the four subscales of the measure. From there, frequencies were run to identify the levels of Emotional Intelligence for the EMTs in this study. The scoring breakdown was presented for the CD-RISC, with frequencies run to identify the levels of resiliency for the EMTs in this study. To evaluate for Attachment, the subscales were presented with each of the items comprising those subscales, after which frequencies were run to identify the attachment styles and their levels utilized by the private sector EMTs in this study. For Research Question Five, a Multiple Linear Regression 
was run in order to identify the nature of the relationship between PTSD severity and Emotional Intelligence, Coping Skills, Resiliency, and Attachment, and to identify the unique contribution of each of the variables (coping techniques, emotional intelligence, resiliency, and attachment) in the explanation of PTSD symptomatology in EMTs. 


\section{CHAPTER 4}

\section{RESULTS OF THE STUDY}

\section{Introduction}

The purpose of this study was to examine the relationship between coping skills, emotional intelligence, attachment, and resilience on PTSD symptoms in private sector Emergency Medical Technicians (EMTs) workers on all levels (EMT-B, EMT-I, and EMT-P), and to understand the experiences of this population as workers in the private sector of the field. These were evaluated through both a quantitative analysis utilizing questionnaires that specifically measure these variables, and through a qualitative component that utilized seven questions as they related to the experiences of those working on all three levels as EMTs in private sector of the field.

\section{Sample for Qualitative Portion of the Study}

A total of four individuals responded to the invitation to participate in the qualitative portion of the research study. Of these, one was female, and the other three were male. One participant was Middle Eastern, one was Caucasian/Hispanic, one was Hispanic, and one was Caucasian. The participants ranged in age from 33 to 56 years of age. The total number of years working in the field as an EMT ranged from 10 to 23 years. One participant had worked at the same level for the duration of their time as an EMT, 23 years, and had been an EMT-B their entire EMT career. The range of time the other three participants had worked at their current level as EMT-P's ranged from 2 to 18 years, with the average time worked at their previous level, as EMT-B's, between 3 and 10 years. All participants were married and had children. All four individuals responded to the invitation to provide additional information by contacting this researcher via email, requesting to participate in the individual interview process. The 
interviews for the qualified participants ranged from one to two hours in length, with the average interview time being 90 minutes, and the total interview time for the disqualified participant being 20 minutes in length.

Of the eligible participants, two resided in Michigan, and one resided in Southern California; however, two of the participants' work history was in Southern California, and the third participants' work history was in Michigan. All three of the eligible participants worked in similar areas, with their work covering both large city and suburban areas, and more rural areas as well. The three individuals eligible for the interviews participated in the qualitative portion of the study as a result of responding to an invitation to provide more information following their completion of the quantitative portion of the study, as indicated above. Of the four individuals responding to the invitation to participate in the individual interviews, only three had worked in the field; therefore, only three of the individuals were eligible, leaving the remaining participants all males. For this reason, three male participants were interviewed for this study, with no females participating in this portion of the study. Participant demographic table (Table 11) is shown below: 


\section{Table 11}

Qualitative Study Participant Demographics

\begin{tabular}{|c|c|c|c|c|c|c|c|}
\hline Demographic & & & $\mathrm{n}(3)$ & & $\%$ & $M$ & $S D$ \\
\hline \multirow[t]{2}{*}{ Gender } & Female & & & 0 & 0 & & \\
\hline & Male & & & 3 & 100 & & \\
\hline \multirow[t]{2}{*}{ Age (Range: 20-67) } & & 43 & & 2 & 66.7 & 47.33 & 7.51 \\
\hline & & 56 & & 1 & 33.3 & & \\
\hline \multirow[t]{2}{*}{ Cultural Identity } & "Hispanic & & & 2 & 66.7 & & \\
\hline & "White" & & & 1 & 33.3 & & \\
\hline \multirow[t]{2}{*}{ EMT Level: } & EMT-B & & & 1 & 33.3 & & \\
\hline & EMT-P & & & 2 & 66.7 & & \\
\hline \multicolumn{8}{|l|}{ Time in Field: } \\
\hline \multirow[t]{2}{*}{ Previous Level } & 10-20 Years & & 1 & & 33.3 & 10.33 & 8.99 \\
\hline & $20+$ Years & & 2 & & 66.7 & & \\
\hline \multirow[t]{2}{*}{ Current Level } & 15-20 Years & & 2 & & 66.7 & 19.00 & 2.94 \\
\hline & $20+$ Years & & 1 & & 33.3 & & \\
\hline \multirow[t]{2}{*}{ Total Overall } & $0-5$ & ars & & 2 & 66.7 & 21.67 & 1.89 \\
\hline & $20+$ & ars & & 1 & 33.3 & & \\
\hline
\end{tabular}

\section{Description of the Variables}

\section{Individual Interview Questions}

While reviewing the literature in the field regarding the various EMS populations, it became clear that each EMS entity held intricacies unique to their specific work environment, while still holding similarities with other sectors of the field as well. Because of the paucity of research on private sector EMTs, the qualitative portion of this study was conducted with the intention of going hand-in-hand with the quantitative portion of the study. Therefore, drawing on information provided by private sector EMTs in this researcher's private life, as well as on this researcher's experiences in the lack of recognition and attention provided to the private sector of the field as a potential factor in the experience of PTSD for private sector EMTs, a total of seven questions were selected for this portion of the study. The intent of this portion of the study was to identify information that would be relevant to private sector EMTs which would not 
be otherwise recognized without an evaluation beyond that obtained through the use of the quantitative surveys. This, then, serves to provide a richer source of information at a greater depth than provided by the quantitative portion solely. The seven questions comprising the qualitative portion are as follows:

1. Describe your work environment as an EMT working in the private sector of the field.

2. What were some of the things that drew you to desire working in this field?

3. Describe what brought you to working in the private sector versus the public sector of the field.

4. In your opinion, what are the differences between working in the private versus the public sector of the field?

5. How does the EMS culture play a role in your experiences as a private sector EMT?

6. In your opinion, is there a difference in community or societal response to you as a private sector EMT versus a public sector EMT? If so, how does the public's response to your work in the private sector impact your work as a private sector EMT?

7. What advice would you give to someone who is considering working in the private sector of the field?

\section{Qualitative Objective}

The objective of the qualitative portion of this study was to identify the experiences of EMTs working in the private sector of the field to evaluate for three factors: 1) Whether there were perceived differences between working in the private versus the public sector of the field;

2) If private sector EMTs express similarities in the role EMS culture plays in their experiences 
as private sector EMTs; and 3) Whether private sector EMTs express perceived differences in community or societal response to their work as opposed to community or societal response to the work of public sector EMTs.

Content analysis was the analysis procedure used to evaluate the qualitative data. Each of the participant interviews were first transcribed, after which the interviews were evaluated through the use of open, line-by-line coding. Following the coding of the interviews, the interviews were axial coded in order to identify for the presence of common themes among the three participants. In addition to coding each of the interviews, this researcher performed memo writing on the transcripts for further evaluation, and to identify any additional themes that may have been missed previously. Following the identification of categories from the transcripts, codes were identified in order to identify any themes that were specific to each of the participants themselves, but which may not have fit into a pattern across the three participants. With one of the goals being to identify both shared and individual experiences among the participants, single participant categories were retained that had been identified among individual participants.

\section{Qualitative Validity}

When examining validity in qualitative research, one must identify whether or not the information obtained is true with regard to the population of interest and with regard to the information obtained. According to Cypress (2017), the standards for reliability and validity in qualitative research was set by Guba and Lincoln (1989) with their proposal of four guidelines. These consisted of what they termed credibility, transferability, dependability, and confirmability (Lincoln \& Guba, 1989). Credibility refers to the establishment of results that are credible from the perspective of the participant, and is analogous to internal validity in quantitative research (Trochim, 2020). Transferability, which is analogous to external validity in quantitative research 
refers to the generalizability of the results (Trochim, 2020). Dependability, which is analogous to reliability, refers to the ability to replicate or repeat the results of the study, with the researcher being responsible for describing how changes may occur within the setting of the particular research context that could affect how future research may be approached in replication studies (Trochim, 2020). Finally, confirmability, which is analogous to objectivity, refers to the ability for others to corroborate or to confirm the research results (Trochim, 2020).

Other researchers propose that rigor, or the "quality or state of being very exact, careful, or thorough and accurate" (Oxford English Dictionary, 2013 as cited in Cypress, 2017, p. 254). Trustworthiness is another aspect that is considered in qualitative research, and is considered to be the authenticity, quality, and truthfulness of the research findings, and is related to the amount of confidence or degree of trust that the readers of those research findings may have in the results (Cypress, 2017). In addition to these, validity is of concern in qualitative research, just as it is in quantitative research, with validity being concerned with both the accuracy of the research findings as well as the truthfulness of those findings (Van Manen, 1990b as cited in Cypress, 2017). Because qualitative research seeks to understand the phenomena of interest as expressed from the viewpoint of the participant without any manipulation of that phenomena, the data is grounded in that process. This assists in providing both reliability and validity, as this lends itself to the rigor of the study process and findings (Cypress, 2017). Smith (1989) made the argument that it is this expression of the viewpoint of the participant that lends itself to the truth of that individual's experience or trustworthiness, which then becomes a social agreement, and therefore, reliable and valid for that experience.

Validity within this study was achieved through the use of researcher/participant consensus with regard to the information as provided by the participants. In other words, this 
researcher verified the information as presented by each participant and what the definition of the terminology entailed as used by each participant without identifying this information in a specific manner to be used later in the coding process by first verifying the accuracy of the terminology with each of the participants. By allowing each participant to be a part of the formulation of constructs related to their experiences, construct validity was ensured, which then led to the formulation and use of quality criteria in being able to both explore and to describe the criteria that was most relevant to each of the research participants. By default, this then also ensured rich and quality constructs and quality criteria for the study itself. Because of this process, trustworthiness was demonstrated through the use of each of the combination of each of these components together, without placing any personal meaning or interpretation of this researcher on each of the terms, concepts, and constructs, but instead, the reliance of each of these was used in such a way as to permit for the information obtained from the participants to fit them and their experiences.

\section{Findings/Results}

\section{Participants}

There were three qualified research participants for this portion of the study. Pseudonyms were used to protect the identity of the participants. Below is an introduction to the qualitative study participants.

\section{Fred}

Fred is a 56-year-old Hispanic male who has worked as an EMT-Basic for 23 years. He has been a Field Training Officer (FTO) for 15 years, and because of his seniority and his professional expertise, he has spent the last 5 years training all new and incoming field employees, as well as being a CPR instructor for 6 years. 


\section{Drake}

Drake is a 43-year-old Caucasian male who has worked in the field for 23 years. He worked as an EMT-Basic for five years, after which time he became an EMT-Paramedic. He has worked as an EMT-P for 18 years, with his total time in the field being 23 years. His career in the EMS (Emergency Medical Services) field began while he was in the military. He began working in the private sector of the field once he returned to civilian life.

\section{James}

James is a 43-year-old Hispanic male who worked as an EMT-Basic for three years, after which time he became an EMT-Paramedic. He worked as an EMT-P for 16 years, with his total time in the field being 19 years. While working in the field, he had been a CPR instructor for the last 4 years of his field time, and is now an EMT instructor as of one year ago.

\section{Qualitative Interview Questions and Themes}

\section{Qualitative Interview Question One}

Participants were asked to describe their work environment as an EMT working in the private sector of the field. Participants described their work environment as consisting of long shifts ranging from twelve to twenty-four hours in length, responding to both critical and noncritical calls, with high volumes of calls of up to 55,000 of those calls resulting in transporting of patients, and with the entirety of the shift spent sitting in the ambulance. Respondents reported running calls on a back-to-back basis, without any break in shift for breaks, meals, or even to use the restroom.

\section{Qualitative Interview Question Two}

Participants were asked to describe some of the things that drew them to desire working as an EMT in the field. The responses for this question included statements that they did not 
desire to work in the fire service field due to their enjoyment of running medical calls over that of running fire calls, and due to the fact that fire calls would take precedence over medical calls. They also reported feeling good about the work that they did and feeling competent at it, having the ability to help others, and enjoying the challenges that were inherent in running EMS calls. One participant stated that he enjoyed not having a job that required him to sit in an office, and having a job that gave him an "adrenaline rush."

\section{Qualitative Interview Question Three}

Participants were asked to describe what brought them to working in the private sector versus the public sector of the field. Participants described the process of joining the fire service as being extensive, competitive, consisting of hundreds of applications per opening, and the process being political in nature. They described not wanting to be firefighters due to fire calls taking precedence over medical calls, and enjoying working for the private sector. One participant described the private sector as being the most practical for him due to him "aging."

\section{Qualitative Interview Question Four}

Participants were asked to express their opinion as to what are the differences are between working in the private versus the public sector of the field. The participants all described the private sector not "getting the credit" for calls that they had run, with the public sector receiving that credit, and the lack of recognition for the work that they, as private sector providers, performed. They described doing the same job, and most times, even more than those in the public sector, with the public sector often not showing up on scene until the work was already done, and still receiving the credit for doing the work even though they had not been present on scene. They stated that public sector EMTs were seen as being "hero's" despite the fact that they did less, ran fewer calls, and often never showed up on scene. 
They also described differences in work setting, with those in the public sector having a station to house them during their shifts, while they sat in their ambulances for the entire twelve to twenty-four hours of their shifts. They reported that they ran along with the public sector EMTs dangerous calls and calls in which there were hazardous conditions present, without them (the private sector EMTs) receiving any recognition or thanks for their service under the same conditions as the public sector EMTs. Another difference noted was that they were the primary providers for running and transporting all types of calls, including emergency and nonemergency transports, as well as long distance transports, which was not part of the job description for fire service EMTs. Their work day was described as a "constant running of one call after another," with no down time, and they described the outcome as being one that "wore [them] down."

Pay and retirement were other notable differences, with the private sector having limited options for retirement, and with private sector EMTs receiving much less pay than those in the public sector. This difference was also noted in terms of the equipment that was available for them to perform their jobs, with those in the fire service having much newer and better equipment at their disposal due to the public funding that they received.

Finally, they reported that public sector EMTs had safe and secure locations to take breaks in, that they had the option as to whether to run calls or not, with them often being able to cover calls from their post (at the fire station), and that they had unions, funding, and structure coving them in their jobs.

\section{Qualitative Interview Question Five}

Participants were asked to describe how the EMS culture plays a role in their experiences as a private sector EMT. The participants described a change in the field once the public sector 
made the decision to place EMS personnel on their units, resulting in a usurping of the field by the public sector. One participant (Fred) stated that the public sector "always wanted control and they were given it." The public sector was also described as having chaplains and other resources to assist them in dealing with traumatic calls, which the private sector did not have. He described the EMS culture as follows: "The private culture was that you ran your calls as fast as you could, clear the hospital as fast as you could, and get that next call whether it was a 911 call or interfacility transport. We had no time to debrief or de-stress after each call." Drake stated, "To be able to have a place to get out of the truck, and just turn off a second or two may have been a difference-maker for me. The constant push of the system, driven by profit, means less people on trucks, and more call volume for the crews, multiplying the effect of those horrible calls that cause problems." James also stated, "In the public sector, everyone basically knows each other and watches out for one another. In the private sector, there is a strong distrust for management and corporate.”

\section{Qualitative Interview Question Six}

Participants were asked to describe in their opinion, whether there is a difference in community or societal response to them as a private sector EMTs versus a public sector EMTs, and if so, how the public's response to their work in the private sector impacts their work as a private sector EMTs. All three of the participants described not being taken seriously as private sector EMTs, with the public seeing them as "ambulance drivers" instead of EMS personnel with expertise and skills. They reported not being acknowledged by the public as being present at major traumatic events, and not being given any credit for the work that they often did hand-inhand with the public sector EMTs. Drake stated, “The public doesn't realize we are a private company." 
Participants also described a lack of respect to them not only by the public, but by members of the public sector providers as well. They reported that this led to disdain for the public sector providers, and to conflict on scene when the public sector would finally show up and then want to take over the scene. The lack of public recognition for their contribution and work led to many EMTs in the private sector experiencing feelings of disenfranchisement.

\section{Qualitative Interview Question Seven}

Participants were asked to describe what advice they would you give to someone who is considering working in the private sector of the field. Respondents stated the need to know evaluate and know why the individual desires to work in the private sector of the field, and to have a full understanding that part of their goals should not include a desire to make money. They also advised that one should understand that family, personal health, and job recognition will not be viewed as priority, but that these will be significantly affected by working in the private sector. They stated that getting into the private sector as an EMT should be done because one wants to help others. They also advised that one should have a reasonable outlet to deal with job stressors, and to "seek help" for "bad days."

\section{Additional Information}

Finally, participants were given the option of sharing additional information. Fred reported, "This field has changed who I have become and has desensitized me from emotions and caring for others." Drake stated, "Some departments do a phenomenal job of crushing the stigma that comes with seeking help in public services/EMS, but others suck so bad at it that they make people worse." And James stated, "Since the survey is about PTSD, it is worth noting that the private sector has a very weak plan overall in dealing with PTSD and other mental health issues. This seems to be consistent among most private EMS providers." 


\section{Categories and Themes}

Following the evaluation of the interviews utilizing line-by-line coding and the grouping of these into categories, overlapping areas were identified, after which the categories were grouped into themes, resulting in five overarching themes and two subcategories. Identified categories were Work Environment, Work Quality/altruism, disenfranchisement, resiliency, and contributors to PTSD. Of these, Work Quality had Altruism as a subcategory, and Disenfranchisement had Societal Acknowledgment as a sub-category. These categories and themes coincide with the results of the quantitative results, in that they confirm the foundation or base of individuals' experiences that the research has documented as contributory in leading to PTSD.

\section{Theme 1: Work Environment}

The work environment described by the participants was a difficult one at best, with the private sector EMTs spending their entire shift sitting in one place: in either the driver's side or the passenger side of the front of the ambulance, when they were not running calls. Fred stated, "I worked 12 and 24 hour shifts on a 3-on-4-off schedule on a rig." The work environment described was dominated by the constant running of back-to-back calls, into high-volume areas. Drake stated, "I would be sitting in an ambulance for 12 hours a day, getting pounded by calls in a meat grinder of a city. The area I worked was very high volume. 55,000+ transports a year." Fred also reported working in hazardous and dangerous conditions, running both emergency and non-emergency calls. He stated, “The work environment included 911 responses that ranged from non-critical calls to extremely critical calls. There were a lot of times that I was exposed to hazards and dangers doing my job running calls back to back at times." 
He also reported that the public sector EMTs had both housing and response options not available to the private sector EMTs, "Fire stays on one location that they are housed in and covers that part only unless a huge response goes out and they may have to hard cover another station; usually they soft cover, as they are close to the area they may have to cover." His description of soft and hard cover was explained as follows: "Hard cover means that they go out and respond directly to the call by having to go on scene; soft cover means that they just listen from the station whether they need to go out or not." Drake stated, "I envied the station life the public medics had. Their representation etc. To be able to have a place to get out of the truck, and just turn off a second or two may have been a difference-maker for me."

\section{Theme 2: Work Quality/Altruism}

Despite the working conditions, all of the research participants described enjoying their work and getting satisfaction from helping others, as well as feeling that they were good at what they did. Fred stated, "While working for a volunteer fire department I found that I enjoyed running medical calls more than fire calls. I felt that I was good at what I did and had the ability to help others when they needed it most. I enjoyed the challenges that were presented during the calls." Drake reported having done medical EMS work while in the military. He reported, "I had also done this in the military, and I liked it then."

\section{Altruism}

James stated that his primary reason for becoming an EMT was to help people. He stated, "Helping people was my primary reason." Fred stated, "I did not want to be a fire firefighter though I could have run calls on a squad. But you are a fire fighter first and medical second, and I did not want that. I believed that helping someone rather than protecting a structure was more important." 


\section{Theme 3: Disenfranchisement}

The theme of disenfranchisement was prevalent throughout the participant interviews. Fred reported, "In the private sector you never get credit. It always goes to the fire fighters and police, even though there were times that fire never showed up and they still got the credit. The public sector feels that they put their lives on the line every call they go on, and so the public sees them as the hero's when the private sector does the same. I have been in many dangerous situations right along with them and not a word or thanks." James expressed, "In the private sector, EMS is beneath fire."

The disenfranchisement could also be seen in the lack that was present for private sector EMTs as opposed to the provisions for the public sector. These included less pay for the same type of work, limited to no retirement benefits, less updated and appropriate resources such as equipment. Fred stated, "The public sector pays more and has more benefits and retirement offerings, where the private has none. Public is funded and gets updated equipment, where private is not, and it all depends on getting your billing paid whether you had the money for upgrading equipment or even pay raises.

\section{Societal Acknowledgment}

The lack of societal acknowledgement was a piece of the disenfranchisement, as expressed by the research participants. Both Fred and Drake stated, "The private sector is not taken serious or sees us as being skilled. We get called Ambulance drivers and not taken serious!" James expressed similar sentiment, "There is definitely a societal difference in how private versus public EMS providers are viewed. Public service providers are viewed as real paramedics, and EMTs and private providers are often viewed as drivers." 


\section{Theme 4: Resiliency}

Resiliency was also a theme among several of the participants, with Fred expressing the ability to continue doing his job despite the lack of societal recognition. He stated, "I did not let it affect how I did my job and how I treated my patients." James expressed similar abilities, "It never really impacted me personally because I know the truth and I don't put a lot of stock in what people say or think. However, I know for a fact that it did bother many of my colleagues over the years."

\section{Theme 5: Contributors to PTSD}

A number of statements by the participants were identified as contributing to the possible expression of PTSD symptomatology. These included the expression of their non-stop work environment, as reflected in Theme 1, above, as well as running traumatic calls. This was expressed most overtly by Fred, "The work environment included 911 responses that ranged from non-critical calls to extremely critical calls. There were a lot of times that I was exposed to hazards and dangers doing my job running calls back to back at times." Drake also expressed the same in his statements that he experienced, "sitting in an ambulance for 12 hours a day, getting pounded by calls in a meat grinder of a city. The area I worked was very high volume. 55,000+ transports a year."

It also included the sentiment that there was no down time for these private sector EMTs, as expressed by Drake, "It was different for us. I envied the station life the public medics had. Their representation, etcetera. To be able to have a place to get out of the truck, and just turn off a second or two may have been a difference-maker for me. The constant push of the system, driven by profit, means less people on trucks, and more call volume for the crews, multiplying the effect of those horrible calls that cause problems. Being the black sheep of the public 
services is not an easy job. Then, add in the time from home, holidays, birthdays, crappy partners, the constant grind of the system. That's how you get untreated mental health issues." Fred stated, "They have chaplains and other programs that aide in their critical incidents whereas the private sector was not afforded that. The private culture was that you ran your calls as fast as you could, clear the hospital as fast as you could, and get that next call whether it was a 911 call or interfacility transport. We had no time to debrief or de-stress after each call. There is pretty much no outlet for this in the private sector as there is for the public. This field has changed who I have become and has desensitized me from emotions and caring for others." James concurred with this, stating, "The private sector has a very weak plan overall in dealing with PTSD and other mental health issues. This seems to be consistent among most private EMS providers."

\section{Sample for Quantitative Portion of the Study}

A total of 300 Emergency Medical Technicians (EMTs) from the United States were sought to be recruited for participation in this study. Participants were recruited through the use of a research study invitation through the use of social media sites such as Facebook, by word of mouth, and through the Michigan State DHHS. Although the sample size for this study was estimated through power analysis with a total estimated sample size of 300 participants, there were only a total of 113 respondents, with 57 responses by public sector EMTs and 56 responses by private sector EMTs, 3 outliers, and 3 incomplete responses, leaving only 50 qualified respondents who met research eligibility criteria, and were able to proceed with and complete the survey instruments. Due to the limited number of eligible participants, the design of the study was changed to a mixed-methods design, with a qualitative component implemented for the purpose of identifying uniqueness's appropriate to this population. 


\section{Participant Demographics}

Of the 50 eligible participants, $46 \%$ were female $(n=23)$ and $54 \%$ were male $(n=27)$. The participants ranged in age from 20 to 67 years of age, with a mean of 44.44 years of age and a standard deviation of 13.36. The cultural background of the participants per participant response were indicated as follows: "American" $(n=5 ; 10 \%)$, “Caucasian/European Descent" $(n$ $=20 ; 40 \%)$, "Hispanic" $(n=2 ; 4 \%)$, "Native American" $(n=1 ; 2 \%)$, "Traditional" $(n=1 ; 2 \%)$, "Other" $(n=1 ; 2 \%)$, and "White" $(n=20 ; 40 \%)$. Breakdown of EMT levels included EMTBasic's $(30 \%, n=15)$, EMT-Intermediate's $(4 \%, n=2)$, and EMT-Paramedic's $(66 \%, n=33)$. Time worked in the field was represented by actual years, with the number of years at the current EMT level (EMT-B, EMT-I, EMT-P) ranged from 6 months to 39 years, with a mean of 11.75 years, and a standard deviation of 14.55 years; time worked in the field at a previous EMT level ranged from 1 to 34 years, with a mean of 6 years and a standard deviation of 12.5 years; and with the total number of years worked overall as an EMT ranging from 1 to 41 years, with a mean of 12.5 years and a standard deviation of 12.76 years. Table 12 presents the demographic characteristics of the quantitative participants. 
Table 12

Sample for Quantitative Portion of the Study

\begin{tabular}{llcccc}
\hline & & $n(50)$ & Percent & $M$ & $S D$ \\
\hline Gender & Female & 23 & 46 & & \\
Age (Range: 20-67) & Male & 27 & 54 & & \\
& $20-29$ & & & 44.44 & 13.36 \\
& $30-39$ & 9 & 18 & & \\
& $40-49$ & 10 & 20 & & \\
& $50-59$ & 11 & 22 & \\
Cultural Identity & 12 & 24 & \\
& 60-69 & 8 & 16 & \\
& "American" & 5 & 10 & \\
& "Caucasian/European Descent" & 20 & 40 & \\
& "Hispanic" & 2 & 4 & \\
& "Native American" & 1 & 2 & \\
& "Traditional" & 1 & 2 & \\
& "Other" & 1 & 2 & \\
EMT Level & "White" & 20 & 40 & \\
& EMT-B & 15 & 30 & \\
& EMT-I & 2 & 4 & \\
& EMT-P & 33 & 66 & \\
& Previous Level & Range: & 6 months - 39 years & \\
& Current Level & Range: & 0 years - & 14 years & \\
& Total Time Overall & Range: & 6 months - & 41 years & \\
& & & &
\end{tabular}

\section{Description of the Variables and Instrument Reliability}

PTSD was the dependent variable for this study, with four independent variables utilized for evaluation: coping, emotional intelligence, attachment, and resilience.

\section{Post-Traumatic Stress Disorder}

The dependent variable in this study was PTSD. The instrument that was used to identify respondent match with PTSD symptoms was the PTSD Checklist, also known as the PCL-5. As shown in Table 13, below, the Cronbach's alpha for the variable, PTSD, was .96 for this study. Cronbach's alpha for each of the criterion were as follows: Criterion $\mathrm{B}=.92$; Criterion $\mathrm{C}=.82$; Criterion $\mathrm{D}=.91 ;$ and Criterion $\mathrm{E}=.86$. 
Table 13

PTSD Reliability Summary Table

\begin{tabular}{lrcc}
\hline & Subscales & Number of Items & Cronbach's Alpha \\
\hline PTSD & & 20 & 0.96 \\
& Criterion B & 5 & 0.92 \\
& Criterion C & 2 & 0.82 \\
& Criterion D & 7 & 0.91 \\
& Criterion E & 6 & 0.86 \\
\hline
\end{tabular}

\section{Coping}

The first independent variable in this study, Coping, was measured by the Brief COPE, which measures coping strategies that represent the individual's way of coping using either their thoughts or their actions when under stress or when in difficult situations. Cronbach's alpha for the variable, Coping, is shown in Table 14, demonstrating a Cronbach's alpha of .90 for Coping, and a Cronbach's alpha of .80 for the subscale, "Use of Emotional Social Support;" .89 for the subscale, "Use of Problem Solving," .81 for the subscale, "Avoidance Coping," and .66 for the subscale, "Use of Positive Thinking" for this study. "Although Use of Positive Thinking" falls below .70, it is just below a normatively acceptable range, and should be interpreted with caution.

\section{Table 14}

Coping Reliability Summary Table

\begin{tabular}{llcc}
\hline \multirow{2}{*}{ Variable } & \multicolumn{1}{c}{ Subscales } & Number of Items & Cronbach's Alpha \\
\hline \multirow{2}{*}{ Coping } & & 28 & 0.90 \\
& & 8 & 0.80 \\
& Use of Emotional Social Support & 4 & 0.89 \\
& Use of Problem Solving & 10 & 0.81 \\
& Avoidance Coping & 6 & 0.66 \\
\hline
\end{tabular}




\section{Emotional intelligence}

The second independent variable, Emotional Intelligence, measured participants' ability to understand emotion, ability to utilize one's own emotion to assist in facilitating the thinking process, and the ability to manage both one's own emotion and others' emotions, including the ability to perceive and express emotion, (Mayer \& Salovey, 1997). This was accomplished through the use of the Schutte Emotional Intelligence Scale (SEIS), which is also known as the Schutte Self-Report Emotional Intelligence Test (SSEIT) and the Self-Report Emotional Intelligence Test (SREIT), and through the use of the four factors of emotional intelligence (Managing Others' Emotions, Managing Own Emotions, Utilization of Emotion, Perception of Emotion) as identified through factor analysis of the SEIS by Ciarrochi, Deane, and Anderson (2002). The stated purpose of the SEIS is to measure three aspects of emotional intelligence: "how one appraises and expresses emotion, the individual's ability to regulate emotion, and how emotion is utilized in the individual's life" (Consortium for Research on Emotional Intelligence in Organizations, 2017, p. 160). As shown in Table 15, Cronbach's alpha for the variable, Emotional Intelligence, was .90, and was .88 for the subscale, "Managing Others' Emotions;" .84 for the subscale, "Utilization of Emotion" and "Managing Own Emotions;" and .78 for the variable, "Perception of Emotion."

\section{Table 15}

Emotional Intelligence Reliability Summary Table

\begin{tabular}{clcc}
\hline Variable & \multicolumn{1}{c}{ Subscales } & Number of Items & Cronbach's Alpha \\
\hline Emotional Intelligence & & 33 & 0.90 \\
& Perception of Emotion & 10 & 0.78 \\
& Managing Own Emotions & 9 & 0.84 \\
& Utilization of Emotion & 8 & 0.84 \\
& Managing Others' Emotions & 6 & 0.88 \\
\hline
\end{tabular}




\section{Attachment}

The third independent variable, attachment, was assessed through the Measure of Attachment Qualities (MAQ), which measures four attachment styles: avoidant attachment, anxious (ambivalent-merger) attachment, ambivalent attachment, and secure attachment. Cronbach's alpha for the variable, Attachment, was.79; .84 for both of the subscales, "Avoidant Attachment” and “Ambivalent-Worry (Ambivalent Attachment);”.79 for the subscale, "Ambivalent-Merger (Anxious Attachment); and .80 for the subscale, "Secure Attachment" as shown in Table 16:

Table 16

Attachment Reliability Summary Table

\begin{tabular}{llcc}
\hline \multicolumn{1}{c}{ Variable } & \multicolumn{1}{c}{ Subscales } & Number of Items & Cronbach's Alpha \\
\hline Attachment & & 14 & 0.79 \\
& Avoidant Attachment & 5 & 0.84 \\
& Ambivalent-Worry (Ambivalent) Attachment & 3 & 0.84 \\
& Ambivalent-Merger (Anxious) Attachment & 3 & 0.79 \\
& Secure Attachment & 3 & 0.80 \\
\hline
\end{tabular}

\section{Resilience}

The final independent variable, resilience, was accomplished through the use of the Connor Davidson - Resilience Scale, which was created to measure resiliency. Cronbach's alpha's for the variable, Resiliency, was .91 for this study, as shown in Table 17:

\section{Table 17}

Resiliency Reliability Summary Table

\begin{tabular}{ccc}
\hline Variable & Number of Items & Cronbach's Alpha \\
\hline Resiliency & 10 & 0.91 \\
\hline
\end{tabular}




\section{Research Questions and Evaluation}

Following were the research questions for the quantitative portion of this study:

1. What is the severity of PTSD among private sector EMTs?

2. Are there differences in the severity of PTSD among EMT-B's, EMT-I's, and EMTP's?

3. What coping strategies do private sector EMTs use?

4. What are their levels and types of emotional intelligence, resilience, and attachment?

5. What is the nature of the relationship between PTSD severity and the following variables: emotional intelligence, coping skills, resiliency, and attachment?

\section{Research Question One}

The first research question sought to identify the severity of PTSD among private sector EMTs. PTSD was measured using the PTSD Checklist for DSM-5 (PCL-5). Severity of PTSD was assessed using two criteria: the total symptom severity score using the PCL-5 (Weathers, Litz, Keane, Palmieri, Marx, \& Schnurr, 2013) and DSM-5 (American Psychiatric Association, 2013) criterion severity scores. Total symptom severity scores are obtained by summing the response score of each of the 20 items of the PCL-5 (Weathers, et al., 2013). Each item is scaled along a 5-point Likert scale: 0-not at all, 1-a little bit, 2-moderately, 3-quite a bit, and 4extremely (Weathers, et al., 2013). Scores of 33 or higher are indicative of PTSD (Weathers, et al., 2013). To determine criterion severity scores, items with responses of 'moderately' or higher are treated as endorsed PTSD symptoms (American Psychiatric Association, 2013). A provisional PTSD diagnosis is then made if the following are met: 1 Criterion B item (Items 15), 1 Criterion C item (Items 6-7), 2 Criterion D items (Items 8- 14) and 2 Criterion E items (Items 15-20) (American Psychiatric Association, 2013). 
Table 18 reports the number and percentage of those categorized as having PTSD based on the cut-off PCL-5 score of 33. Approximately a third $(n=15,30 \%)$ of the EMTs were assumed to have PTSD. The mean total symptom severity score for those identified as having PTSD was $46.47(S D=10.51)$, with scores ranging from 0.00 to 64.00 , while those with no PTSD had a mean of $11.20(S D=9.88)$.

\section{Table 18}

Overall Percentage of PTSD Endorsed Based on PCL-5 Criteria

\begin{tabular}{lcc}
\hline \multicolumn{1}{c}{ Group } & $N$ & $\%$ \\
\hline PTSD (does not meet criteria) & 35 & 70.0 \\
PTSD (meets criteria) & 15 & 30.0 \\
\hline
\end{tabular}

Provisional diagnosis of PTSD based on DMS-5 criteria (1 "B" item, 1 "C" item, 2 "D" items, and 2 "E" items) are reported in Table 18 . Slightly over a third $(n=18,36 \%)$ of the EMTs were identified as having PTSD. This result is consistent with the identification of PTSD when total symptom severity score is used (see Table 19).

\section{Table 19}

Overall Percentage of PTSD Endorsed Based on DSM-5 Criteria

\begin{tabular}{lcc}
\hline \multicolumn{1}{c}{ Group } & $N$ & $\%$ \\
\hline PTSD (does not meet criteria) & 32 & 64.0 \\
PTSD (meets criteria) & 18 & 36.0 \\
\hline
\end{tabular}

Table 20 shows the number of items in each criterion that were endorsed by the EMTs in this study. Endorsed items are symptomatic of PTSD. For example, in Criterion B (Intrusion), $56 \%$ of the EMTs endorsed 1 or more items. Similarly, 64\% of EMTs endorsed 1 or more items in Criterion E (alterations in arousal and reactivity). 
Table 20

Percent of EMTs Endorsing DSM-5 Items

\begin{tabular}{|c|c|c|c|c|c|c|c|c|c|c|}
\hline \multicolumn{11}{|c|}{ Number of Items Endorsed } \\
\hline DSM-5 & Criteria & Item & 0 & 1 & 2 & 3 & 4 & 5 & 6 & 7 \\
\hline Criteria & Theme & \# & & & & & & & & \\
\hline $\mathrm{B}$ & Intrusion & 5 & 44.0 & 22.0 & 6.0 & 6.0 & 8.0 & 14.0 & & \\
\hline $\mathrm{C}$ & Avoidance & 2 & 54.0 & 20.0 & 26.0 & & & & & \\
\hline D & Negative & 7 & 46.0 & 10.0 & 12.0 & 6.0 & 10.0 & 4.0 & 4.0 & 8.0 \\
\hline & Alterations & & & & & & & & & \\
\hline $\mathrm{E}$ & Alterations in & 6 & 36.0 & 16.0 & 8.0 & 8.0 & 16.0 & 12.0 & 4.0 & \\
\hline & $\begin{array}{c}\text { Arousal and } \\
\text { Reactivity }\end{array}$ & & & & & & & & & \\
\hline
\end{tabular}

Tables 21-24 report percent of EMTs who endorsed the items in each criterion. Fifty two percent (52\%) of EMTs endorsed the symptom of 'being super alert, watchful or on guard'

(Criterion E); 40\% endorsed "repeated, disturbing and unwanted memories of the stress

experience" (Criterion B); and 38\% endorsed "avoiding memories, thoughts, or feelings related

to stressful experience" (Criterion C).

\section{Table 21}

Percent of EMTs Endorsing Criterion B Items $(n=50)$

\begin{tabular}{lc}
\hline \multicolumn{1}{c}{ Item } & $\%$ \\
\hline Repeated, disturbing, and unwanted memories of the stressful experience? & 40.0 \\
Feeling very upset when something reminded you of a stressful experience? & 36.0 \\
Repeated, disturbing dreams of a stressful experience? & 30.0 \\
Having strong physical reactions when something reminded you of the stressful experience (for & 26.0 \\
example, heart pounding, trouble breathing, sweating)? & 22.0 \\
Suddenly feeling or acting as if the stressful experience were actually happening again (as if you & \\
were actually back there reliving it)? & \\
\hline
\end{tabular}

\section{Table 22}

Percent of EMTs Endorsing Criterion C Items $(n=50)$

\begin{tabular}{lc}
\hline \multicolumn{1}{c}{ Item } & $\%$ \\
\hline Avoiding memories, thoughts, or feelings related to the stressful experience? & 38.0 \\
Avoiding external reminders of the stressful experience (for example, people, places, & 34.0 \\
conversations, activities, objects, or situations)? & \\
\hline
\end{tabular}


Table 23

Percent of EMTs Endorsing Criterion D Items $(n=50)$

\begin{tabular}{lc}
\hline \multicolumn{1}{c}{ Item } & $\%$ \\
\hline Feeling distant or cut off from other people? & 36.0 \\
Loss of interest in activities that you used to enjoy? & 32.0 \\
Trouble experiencing positive feelings (for example, being unable to feel happiness or have loving & 30.0 \\
feelings for people close to you)? & 28.0 \\
Having strong negative beliefs about yourself, other people, or the world (for example, having & \\
thoughts such as: I am bad, there is something seriously wrong with me, no one can be trusted, the & \\
world is completely dangerous)? & 26.0 \\
Having strong negative feelings such as fear, horror, anger, guilt, or shame? & 22.0 \\
Trouble remembering important parts of the stressful experience? & 18.0 \\
Blaming yourself or someone else for the stressful experience or what happened after it?
\end{tabular}

Table 24

Percent of EMTs Endorsing Criterion E Items $(n=50)$

\begin{tabular}{lc}
\hline \multicolumn{1}{c}{ Item } & $\%$ \\
\hline Being "superalert" or watchful or on guard? & 52.0 \\
Trouble falling or staying asleep? & 38.0 \\
Irritable behavior, angry outbursts, or acting aggressively? & 34.0 \\
Having difficulty concentrating? & 34.0 \\
Feeling jumpy or easily startled? & 32.0 \\
Taking too many risks or doing things that could cause you harm? & 14.0 \\
\hline
\end{tabular}

\section{Research Question Two}

The second research question sought to identify the differences in PTSD severity among the three levels of EMTs: EMT-B's; EMT-I's; and EMT-P's. There were only two EMT-I participants; therefore, they were excluded from further analysis. The Independent Sample T-

Test was conducted to determine differences between the two groups of EMTs (EMT-B's and EMT-P's) on the severity of PTSD.

Skewness of the PTSD scores for EMT-B's is 0.29 and 1.04 for the EMT-P's. Homogeneity of variance assumption was met $(F=0.32, p=0.57)$. Table 25 reports the results of the independent samples t-test, which shows no significant differences between the scores for 
EMT'B's $(M=25.93, S D=20.50)$ and EMT-P's $(M=19.03, S D=18.65) ; t(46)=1.15, p=$ $.255, d=0.35)$. The magnitude of the group differences is small (Cohen's $d=.35$ ).

\section{Table 25}

EMT Group Differences on PCL-5 Scores

\begin{tabular}{cccccccc}
\hline Group & $N$ & $M$ & $S D$ & $t$ & $d f$ & $p$ & $E S(d)$ \\
\hline EMT-B & 15 & 25.93 & 20.50 & 1.153 & 46 & .255 & 0.35 \\
EMT-P & 33 & 19.03 & 18.65 & & & & \\
\hline
\end{tabular}

Differences in the proportion of EMT groups who met PCL-5 criteria for PTSD (i.e. total severity score of 33 or higher) are reported in Table 26 . Based on this cut-off score, approximately $21 \%(n=7)$ of EMT-P's were classified as having PTSD compared to $40 \%(n=$ 6) of EMT-B's. The Chi-square result indicate no significant differences in the percentage of EMT-B's and EMT-P's who met PCL-5 total severity score criteria for PTSD $(\chi 2=1.84, d f=1$, $p=.18, \mathrm{~V}=.20)$.

\section{Table 26}

EMT Group Differences on PCL-5 Cut-Off Scores

\begin{tabular}{|c|c|c|c|c|c|c|}
\hline Group & $\begin{array}{c}\text { No PTSD - } \\
\text { Does Not Meet PCL-5 Criteria }\end{array}$ & $\begin{array}{c}\text { PTSD- } \\
\text { Meets PCL-5 Criteria }\end{array}$ & $\chi^{2}$ & $d f$ & $p$ & V \\
\hline EMT-B & (9)60.00 & $6(40.00)$ & 1.84 & 1 & .18 & .20 \\
\hline EMT-P & $26(78.80)$ & $7(21.20)$ & & & & \\
\hline
\end{tabular}

The proportion of EMTs who met PTSD classification based on DSM-5 criteria (i.e. 1 "B" item; 1 "C" item; 2 "D" items; 2 "E" items) are shown in Table 27. Six (40.0\%) EMT-B's met PTSD criteria, while 10 (30.3\%) EMT-P's met criteria. The Chi-Square test indicates no significant differences in proportions between EMT-B and EMT-P groups $(\chi 2=0.44, d f=1, p=$ $.51, \mathrm{~V}=.10)$. This result is consistent with the proportion of EMTs who met PTSD criteria based on PCL-5 total severity score criterion (see Table 26). 


\section{Table 27}

EMT Group Differences on DSM-5 Criteria: 1 "B" item; 1 "C" item; 2 "D" items; 2 " $E$ " items

\begin{tabular}{ccccccc}
\hline Group & $\begin{array}{c}\text { No PTSD - } \\
\text { Does Not Meet DSM-5 } \\
\text { Criteria }\end{array}$ & PTSD- & $\chi^{2}$ & $d f$ & $p$ & $V$ \\
& $9(60.0)$ & & & & \\
\hline EMT-B & $23(69.7)$ & $6(40.0)$ & 0.44 & 1 & .509 & .095 \\
EMT-P & $10(30.3)$ & & & & \\
\hline
\end{tabular}

The number and percentage of EMTs who met PTSD symptoms based on DSM-5 criteria are summarized in Table 28. In this table, 10 (66.7\%) of EMT-B's and $16(48.5 \%)$ of EMT-P's endorsed (moderately or higher) one or more items in Criterion B. For Criterion C, eight (53.3\%) of EMT-B's, and 13 (39.4\%) EMT-P's endorsed (moderately or higher) one or more items For Criterion D, eight (53.3\%) of EMT-B's, and 12 (36.4\%) EMT-P's endorsed (moderately or higher) two or more items. For Criterion E, nine (60.0\%) of EMT-B's, and 13 (39.4\%) EMT-P's endorsed (moderately or higher) two or more items. The Chi-Square tests of independence indicate that there are no significant differences in the proportion of EMT-B's and EMT-P's who endorsed the number of items that would satisfy DSM-5 PTSD criteria.

\section{Table 28}

Comparison of EMT Groups Meeting Symptoms Based on DSM-5 Criterion

\begin{tabular}{cccccccc}
\hline Criterion & Group & Not Meet Criteria & Meets Criteria & $\chi^{2}$ & $d f$ & $p$ & $V$ \\
\hline B & EMT-B & $5(33.3)$ & $10(66.7)$ & 1.37 & 1 & .35 & .24 \\
& EMT-P & $17(51.5)$ & $16(48.5)$ & & & & \\
& & & & & & & \\
$\mathrm{C}$ & EMT-B & $7(46.7)$ & $8(53.3)$ & 0.81 & 1 & .37 & .13 \\
& EMT-P & $20(60.6)$ & $13(39.4)$ & & & & \\
& & & & & & & \\
D & EMT-B & $7(46.7)$ & $8(53.3)$ & 1.22 & 1 & .27 & .16 \\
& EMT-P & $21(63.6)$ & $12(36.4)$ & & & & \\
& & & & & & & \\
E & EMT-B & $6(40.0)$ & $9(60.0)$ & 1.76 & 1 & .18 & .19 \\
& EMT-P & $20(60.6)$ & $13(39.4)$ & & & & \\
\hline
\end{tabular}




\section{Research Question Three}

The third research question sought to identify the coping strategies that are used by private sector EMTs. Coping strategies were measured using the Brief COPE which measures four coping strategies: Use of Emotional Social Support; Use of Problem Solving; Avoidance Coping; and Use of Positive Thinking. Descriptive statistics for each of the four coping strategies are reported in Table 29. EMTs use Positive Thinking $(M=2.29)$, Problem Solving $(M=2.09)$, Emotional Social Support $(M=2.02)$, and Avoidance Coping $(M=1.58)$ respectively to cope with stressful situations. However, on average, they only use these coping strategies "a little bit." Tables 30-33 report item statistics for each of the strategy.

\section{Table 29}

Descriptive Statistics of Coping Strategies $(n-50)$

\begin{tabular}{lccccc}
\hline \multicolumn{1}{c}{ Coping Strategy } & Min & Max & $M$ & $S D$ & Skewness \\
\hline Use of Positive Thinking & 1.00 & 4.00 & 2.29 & 0.64 & 0.43 \\
Use of Problem Solving & 1.00 & 4.00 & 2.09 & 0.91 & 0.43 \\
Use of Emotional Social Support & 1.00 & 3.43 & 2.02 & 0.66 & 0.17 \\
Use of Avoidance Coping & 1.00 & 2.90 & 1.58 & 0.52 & 0.78 \\
\hline
\end{tabular}

As stated above, Tables 30-33 provide information on the item statistics per coping strategy. Table 30 indicates that the most utilized coping strategy is positive thinking, with "accepting the reality of the fact that the traumatic event has happened" $(M=3.00)$ being the most utilized of the positive thinking strategies, while their least utilized coping strategy, avoidance coping, indicates that their least used coping strategy is in "doing something to think about the traumatic event less, such as going to movies, watching TV, reading, daydreaming, sleeping, or shopping" $(M=1.98)$. 


\section{Table 30}

Positive Thinking Item Statistics $(n=50)$

\begin{tabular}{lcc}
\hline \multicolumn{1}{c}{ Item } & $M$ & $S D$ \\
\hline I've been accepting the reality of the fact that it has happened. & 3.00 & 1.05 \\
I've been learning to live with it. & 2.66 & 1.02 \\
I've been trying to see it in a different light, to make it seem more positive. & 2.22 & 1.07 \\
I've been looking for something good in what is happening. & 2.14 & 1.01 \\
I've been making jokes about it. & 2.02 & 1.08 \\
I've been making fun of the situation. & 1.70 & 1.02 \\
\hline
\end{tabular}

\section{Table 31}

Problem Solving Item Statistics $(n=50)$

\begin{tabular}{lcc}
\hline \multicolumn{1}{c}{ Item } & $M$ & $S D$ \\
\hline I've been taking action to try to make the situation better. & 2.24 & 1.12 \\
I've been thinking hard about what steps to take. & 2.08 & 1.08 \\
I've been concentrating my efforts on doing something about the situation I'm in. & 2.04 & 1.01 \\
I've been trying to come up with a strategy about what to do. & 1.98 & 0.96 \\
\hline
\end{tabular}

\section{Table 32}

Use of Emotional Social Support Item Statistics $(n=50)$

\begin{tabular}{lcc}
\hline \multicolumn{1}{c}{ Item } & $M$ & $S D$ \\
\hline I've been getting comfort and understanding from someone. & 2.12 & 0.98 \\
I've been praying or meditating. & 2.10 & 1.23 \\
I've been trying to find comfort in my religion or spiritual beliefs. & 2.10 & 1.29 \\
I've been expressing my negative feelings. & 2.08 & 0.92 \\
I've been getting emotional support from others. & 2.06 & 0.99 \\
I've been getting help and advice from other people. & 2.04 & 0.97 \\
I've been trying to get advice or help from other people about what to do. & 1.90 & 0.95 \\
I've been saying things to let my unpleasant feelings escape. & 1.84 & 0.87 \\
\hline
\end{tabular}




\section{Table 33}

Avoidance Coping Item Statistics $(n=50)$

\begin{tabular}{lcc}
\hline \multicolumn{1}{c}{ Item } & $M$ & $S D$ \\
\hline I've been doing something to think about it less, such as going to movies, watching & & \\
TV, reading, daydreaming, sleeping, or shopping. & 1.98 & 1.04 \\
I've been criticizing myself. & 1.94 & 0.98 \\
I've been turning to work or other activities to take my mind off things. & 1.90 & 0.99 \\
I've been using alcohol or other drugs to make myself feel better. & 1.62 & 0.95 \\
I've been giving up trying to deal with it. & 1.58 & 0.76 \\
I've been using alcohol or other drugs to help me get through it. & 1.54 & 0.95 \\
I've been blaming myself for things that happened. & 1.48 & 0.86 \\
I've been giving up the attempt to cope. & 1.32 & 0.68 \\
I've been saying to myself "this isn't real." & 1.28 & 0.54 \\
I've been refusing to believe that it has happened. & 1.20 & 0.53 \\
\hline
\end{tabular}

Multivariate analysis of variance (MANOVA) was used to determine differences between EMT-B's and EMT-P's on a linear combination of the four coping strategies. Table 34 provides group means and standard deviations for each of the four coping strategies.

\section{Table 34}

Group Means and Standard Deviations of Coping Strategies

\begin{tabular}{llccc}
\hline Coping Strategy & Group & $M$ & $S D$ & $n$ \\
\hline Emotional Social Support & EMT-B & 2.01 & 0.84 & 15 \\
& EMT-P & 2.03 & 0.59 & 33 \\
Problem Solving & EMT-B & 2.33 & 0.94 & 15 \\
& EMT-P & 1.00 & 0.90 & 33 \\
Avoidance Coping & EMT-B & 1.65 & 0.38 & 15 \\
& EMT-P & 1.53 & 0.57 & 33 \\
Positive Thinking & EMT-B & 2.56 & 0.51 & 15 \\
& EMT-P & 2.12 & 0.63 & 33 \\
\hline
\end{tabular}

Skewness statistics range from a low of .17 for Emotional Social Support to a high of .78 for Avoidance Coping, suggesting that coping strategies may be deemed normally distributed. Equality of variance-covariance matrices assumption is met $($ Box's $M=12.55, F(10,3572.52)=$ $1.10, p=.353)$. Bartlett's test of sphericity suggests that there is sufficient inter-correlation among the dependent variables $\left(\chi^{2}=72.17, d f=9, p<.001\right)$. MANOVA indicates that there are 
no significant differences between the two groups of EMTs (EMT-B's and EMT-P's) on a linear combination of the four coping strategies (Wilk's lambda $=0.83, F(4,43)=2.20, p=.085$, eta squared $=.17)$.

\section{Research Question Four}

Research question four sought to identify the levels and types of emotional intelligence, resilience, and attachment used by private sector EMTs.

\section{Emotional intelligence}

For this variable, The Schutte Emotional Intelligence Scale (SEIS) was used with the interpretation of the entire scale, as based upon the recommendation of Salovey and Mayer (1990), who are the creators of the SEIS. Salovey and Mayer (1990) set three ranges and interpretations for the SEIS, as shown in Table 35, which also demonstrates that the largest percentage of EMTs $(n=29 ; 58 \%)$ fell into the average range on emotional intelligence.

However, approximately $30 \%(n=15)$ of EMTs scored in the unusually high range on emotional intelligence. This indicates that, while the greatest percentage of EMTs do not have any greater ability to understand their own or others' emotions than others in society, one-third of EMTs have a higher than average ability to understand their own or others' emotions than others in society.

\section{Table 35}

Schutte Emotional Intelligence Scale (SEIS) Scoring Breakdown $(n=50)$

\begin{tabular}{lclcc}
\hline \multicolumn{1}{c}{ Score Definition } & Score Range & \multicolumn{1}{c}{ Interpretation } & $n$ & $\%$ \\
\hline Unusually Low & $33-110$ & Lower than average emotional intelligence & 6 & 12 \\
Normative/Average & $111-137$ & Average emotional intelligence & 29 & 58 \\
Unusually High & $138-165$ & Higher than average emotional intelligence & 15 & 30 \\
\hline
\end{tabular}


The SEIS was also categorized into the four subscales of emotional intelligence (Perception of Emotion; Managing Own Emotions; Managing Others' Emotions; Utilization of Emotion) as proposed by the authors (Ciarrochi, Deane, \& Anderson, 2002). Table 36 indicates that EMTs demonstrated the greatest strength in their ability to manage theirs and others' emotions (Managing (their) Own Emotions: $M=3.88$; Managing Others' Emotions: $M=3.85$ ), with the ability to perceive emotions falling very closely behind (Perception of Emotion: $M=$ 3.80). Their weakest area was in their ability to utilize emotions (Utilization of Emotion: $M=$ 3.69).

\section{Table 36}

Descriptive Statistics of Emotional Intelligence Subscales ( $n-50)$

\begin{tabular}{lccccc}
\hline \multicolumn{1}{c}{ Coping Strategy } & Min & Max & $M$ & $S D$ & Skewness \\
\hline Managing Own Emotions & 2.44 & 5.00 & 3.88 & .64 & -.313 \\
Managing Others' Emotions & 2.88 & 5.00 & 3.85 & .52 & .354 \\
Perception of Emotion & 2.20 & 4.90 & 3.80 & .65 & -.286 \\
Utilization of Emotion & 1.83 & 5.00 & 3.69 & .65 & -.553 \\
\hline
\end{tabular}

Tables 37-40 provide information on the item statistics per subscale. Table 37, which addresses their ability to manage their own emotions, shows that EMTs managed their own emotions by recalling other times they had overcome obstacles when faced with new obstacles $(M=4.22)$, and through the use of believing that they will do well on the things they try $(M=$ 4.22); however, they were the weakest in knowing how to make positive emotions last $(M=$ 3.40). Table 38 addresses their ability to manage others' emotions, which demonstrates that their greatest skill was in getting others to confide in them $(M=4.38)$, while their weakest area was in the ability to share in the experiences of others as their own $(M=2.82)$.

Table 39 indicates that their greatest skill in the area of the perception of emotions was in the ability to recognize the emotions that people are feeling just by looking at their facial 
expressions $(M=4.26)$, but that they are weakest in the ability to be aware of the non-verbal messages that they are sending to others $(M=3.22)$. Table 40 demonstrates the area that the EMTs are the weakest in (Utilization of Emotion), showing that their greatest ability in this area was in the ability to evaluate what is important and what is not important $(M=4.22)$, and their weakest ability was being able to see new possibilities when their mood changes $(M=3.04)$.

\section{Table 37}

Managing Own Emotions Item Statistics $(n=50)$

\begin{tabular}{lcc}
\hline \multicolumn{1}{c}{ Item } & $M$ & $S D$ \\
\hline When I am faced with obstacles, I remember times I faced similar obstacles and & 4.22 & 0.79 \\
overcame them. & & \\
I expect that I will do well on most things I try. & 4.22 & 0.73 \\
When I am faced with a challenge, I give up because I believe I will fail. & 4.10 & 1.13 \\
I seek out activities that make me happy. & 4.02 & 1.15 \\
I use good moods to help myself keep trying in the face of obstacles. & 3.78 & 0.86 \\
I expect good things to happen. & 3.76 & 1.13 \\
I motivate myself by imagining a good outcome to tasks I take on. & 3.74 & 1.08 \\
I have control over my emotions. & 3.72 & 1.09 \\
When I experience a positive emotion, I know how to make it last. & 3.40 & 1.09 \\
\hline
\end{tabular}

\section{Table 38}

Managing Others' Emotions Item Statistics $(n=50)$

\begin{tabular}{lcc}
\hline \multicolumn{1}{c}{ Item } & $M$ & $S D$ \\
\hline Other people find it easy to confide in me. & 4.38 & 0.67 \\
I compliment others when they have done something well. & 4.30 & 0.54 \\
I help other people feel better when they are down. & 4.14 & 0.67 \\
I know when to speak about my personal problems to others. & 4.10 & 0.91 \\
I present myself in a way that makes a good impression on others. & 4.04 & 0.86 \\
I like to share my emotions with others. & 3.72 & 1.09 \\
I arrange events others enjoy. & 3.30 & 1.43 \\
When another person tells me about an important event in his or her life, I almost & 2.82 & 1.19 \\
feel as though I experienced this event myself. & & \\
\hline
\end{tabular}




\section{Table 39}

Perception of Emotion Item Statistics $(n=50)$

\begin{tabular}{lcc}
\hline \multicolumn{1}{c}{ Item } & $M$ & $S D$ \\
\hline By looking at their facial expressions, I recognize the emotions people are & 0.75 \\
experiencing. & 4.26 & \\
I can tell how people are feeling by listening to the tone of their voice. & 4.18 & 0.60 \\
I am aware of the non-verbal messages other people send. & 3.96 & 0.95 \\
I am aware of my emotions as I experience them. & 3.92 & 1.03 \\
I find it hard to understand the non-verbal messages of other people. & 3.82 & 1.26 \\
I easily recognize my emotions as I experience them. & 3.82 & 0.98 \\
I know why my emotions change. & 3.68 & 1.11 \\
I know what other people are feeling just by looking at them. & 3.60 & 1.07 \\
It is difficult for me to understand why people feel the way they do. & 3.54 & 1.11 \\
I am aware of the non-verbal messages I send to others. & 3.22 & 1.11 \\
\hline
\end{tabular}

\section{Table 40}

Utilization of Emotion Item Statistics $(n=50)$

\begin{tabular}{lcc}
\hline \multicolumn{1}{c}{ Item } & $M$ & $S D$ \\
\hline Some of the major events of my life have led me to re-evaluate what is important and & 4.22 & 1.04 \\
not important. & 4.20 & 0.81 \\
When I am in a positive mood, solving problems is easy for me. & 4.04 & 0.75 \\
When I am in a positive mood, I am able to come up with new ideas. & 3.56 & 1.16 \\
Emotions are one of the things that make my life worth living. & 3.06 & 1.10 \\
When I feel a change in emotions, I tend to come up with new ideas. & 3.04 & 1.05 \\
When my mood changes, I see new possibilities. &
\end{tabular}

Multivariate analysis of variance (MANOVA) was used to determine differences between EMT-B's and EMT-P's on a linear combination of the four emotional intelligence subscales.

Table 41 provides group means and standard deviations for each of the four emotional intelligence subscales. 


\section{Table 41}

Group Means and Standard Deviations of Emotional Intelligence Subscales

\begin{tabular}{llccc}
\hline Emotional Intelligence Subscale & Group & $M$ & $S D$ & $n$ \\
\hline Managing Own Emotions & EMT-B & 3.76 & 0.62 & 15 \\
& EMT-P & 3.99 & 0.61 & 33 \\
Managing Others' Emotions & EMT-B & 3.80 & 0.38 & 15 \\
& EMT-P & 3.90 & 0.57 & 33 \\
Perception of Emotion & EMT-B & 3.75 & 0.62 & 15 \\
\multirow{3}{*}{ Utilization of Emotion } & EMT-P & 3.85 & 0.67 & 33 \\
& EMT-B & 3.71 & 0.50 & 15 \\
& EMT-P & 3.71 & 0.69 & 33 \\
\hline
\end{tabular}

Skewness statistics ranged from a low of -.55 for Utilization of Emotion to a high of .22 for Managing Others' Emotions, suggesting that the emotional intelligence subscales may be deemed normally distributed. Equality of variance-covariance matrices assumption is met $($ Box's $M=11.60, F(10,3572.52)=1.023, p=42)$. Bartlett's test of sphericity suggests that there is sufficient inter-correlation among the dependent variables $\left(\chi^{2}=68.13, d f=56, p=.005\right)$. MANOVA indicates that there are no significant differences between the two groups of EMTs (EMT-B's and EMT-P's) on a linear combination of the four emotional intelligence subscales (Wilk's lambda $=0.96, F(4,43)=0.47, p=.757$, eta squared $=.04)$.

\section{Resilience}

For this variable, The Connor Davidson - Resiliency Scale (CD-RISC) was used with both the interpretation of the entire scale, and then by quartiles as set forth by Connor and Davidson (2001). Kathryn Connor and Jonathan Davidson, the creators of the CD-RISC (2008) set four ranges and interpretations for the CD-RISC based on the United States population. The mean total resilience score is $3.16(\mathrm{SD}=0.68)$, indicating that it is "often true" that the EMTs in this study were resilient. Table 42 reports the item statistics for resiliency. 
Table 42

Resilience Item Statistics $(n=50)$

\begin{tabular}{lcc}
\hline \multicolumn{1}{c}{ Statement } & $M$ & $S D$ \\
\hline Under pressure, I stay focused and think clearly. & 3.58 & 0.83 \\
I tend to bounce back after illness, injury, or other hardships. & 3.42 & 0.91 \\
I think of myself as a strong person when dealing with life's challenges and difficulties. & 3.37 & 0.89 \\
I believe I can achieve my goals, even if there are obstacles. & 3.37 & 0.82 \\
I can deal with whatever comes my way. & 3.31 & 0.78 \\
I try to see the humorous side of things when I am faced with problems. & 3.18 & 0.84 \\
I am able to adapt when changes occur. & 3.00 & 0.67 \\
I am able to handle unpleasant or painful feelings like sadness, fear, and anger. & 2.94 & 0.92 \\
Having to cope with stress can make me stronger. & 2.77 & 0.87 \\
I am not easily discouraged by failure. & 2.64 & 1.01 \\
\hline
\end{tabular}

Table 43, which also denotes the number and percentages of EMTs in each category, indicates their quartile breakdown of the CD-RISC. This Table demonstrates that the EMTs fell into two categories that are polar opposites; $38 \%$ fell into the highest twenty-five percent of the population, or the most resilient of the population, and $38 \%$ fell into the lowest $25 \%$ of the population, or the least resilient of the population. This indicates that there is an even split between those who are very resilient and those who are very low on resilience, with approximately another third falling in between these two categories of resiliency.

\section{Table 43}

Connor Davidson Resiliency Scale (CD-RISC) Scoring Breakdown

\begin{tabular}{cllcc}
\hline Scoring Range & \multicolumn{1}{c}{ Score Definition } & \multicolumn{1}{c}{ Interpretation } & $n$ & $\%$ \\
\hline $0-29$ & Lowest 25\% of the population & The least resilient of the population & 19 & 38 \\
$30-32$ & Second lowest quartile of the population & The second least resilient of the population & 4 & 8 \\
$33-36$ & Third quartile of the population & The second most resilient of the population & 8 & 16 \\
$37-40$ & Highest 25\% of the population & The most resilient of the population & 19 & 38 \\
\hline
\end{tabular}

Table 44 provides group means and standard deviations for each of the four resiliency scoring ranges based on the Connor and Davidson (2001) quartiles. 


\section{Table 44}

Group Means and Standard Deviations of the CD-RISC Results

\begin{tabular}{llccc}
\hline Resiliency Scoring Interpretation & Group & $M$ & $S D$ & $n$ \\
\hline The least resilient of the population & EMT-B & 22.83 & 3.76 & 6 \\
& EMT-P & 24.18 & 3.63 & 11 \\
The second least resilient of the population & EMT-B & 32.00 & 0.00 & 1 \\
& EMT-P & 30.67 & 0.58 & 3 \\
\multirow{5}{*}{ The second most resilient of the population } & EMT-B & 34.20 & 1.30 & 5 \\
& EMT-P & 34.67 & 0.58 & 3 \\
The most resilient of the population & EMT-B & 38.24 & 0.00 & 3 \\
& EMT-P & 38.34 & 0.72 & 16 \\
\hline
\end{tabular}

To compare resilience scores between EMT-B's and EMT-P's, and independent samples t-test was conducted. Table 45 reports the results of the independent samples t-test, which shows no significant differences between the scores for EMT'B's $(M=30.31, S D=6.97)$ and EMT-P's $(M=32.59, S D=6.78) ; t(46)=-1.07, p=.291, d=0.32)$. The magnitude of the group differences is small (Cohen's $d=.32$ ).

\section{Table 45}

Independent Samples T-Test

\begin{tabular}{cccccccc}
\hline Group & $N$ & $M$ & $S D$ & $t$ & df & $p$ & ES(d) \\
\hline EMT-B & 15 & 30.31 & 6.97 & -1.067 & 46 & .291 & -0.32 \\
EMT-P & 33 & 32.59 & 6.78 & & & & \\
\hline
\end{tabular}

Number and percentage of EMTs who scored in each percentile range for resiliency are summarized in Table 46. In this table, six (40.0\%) of EMT-B's and 11 (33.3\%) of EMT-P's scored in the lowest quartile for resiliency. One (6.7\%) of EMT-B's and three (9.1\%) of EMTP's scored in the second lowest quartile for resiliency. Five (33.3\%) of EMT-B's and three (9.1\%) of EMT-P's scored in the second highest quartile for resiliency. Three (20.0\%) of EMTB's and $16(48.5 \%)$ of EMT-P's scored in the highest quartile for resiliency. The Chi-Square 
tests of independence indicate that there are no significant differences in the proportion of EMT-

B's and EMT-P's on any of the categories of Resiliency.

\section{Table 46}

Chi-Square Test of Independence

\begin{tabular}{ccccc}
\hline & & \multicolumn{3}{c}{ Quartile } \\
Group & $1^{\text {st }}$ & $2^{\text {nd }}$ & $3^{\text {rd }}$ & $4^{\text {th }}$ \\
\hline EMT-B $(n=15)$ & $6(40.0)$ & $1(6.7)$ & $5(33.3)$ & $3(20.0)$ \\
EMT-P $(n=33)$ & $11(33.3)$ & $3(9.1)$ & $3(9.1)$ & $16(48.5)$ \\
\hline$\chi^{2}=5.95, \mathrm{df}=3, p=.114, \mathrm{~V}=35$ & & &
\end{tabular}

\section{Attachment}

For this variable, The Measure of Attachment Qualities (MAQ) was used with the categorization of the four subscales of attachment (Avoidance Attachment; Ambivalence-Worry (Ambivalent) Attachment; Ambivalence-Merger (Anxious) Attachment; Secure Attachment) as proposed by the authors.

\section{Table 47}

Descriptive Statistics of Attachment Styles $(n-50)$

\begin{tabular}{lccccc}
\hline \multicolumn{1}{c}{ Attachment Style } & Min & Max & $M$ & $S D$ & Skewness \\
\hline Secure Attachment & 1.67 & 4.00 & 3.34 & .63 & -0.94 \\
Avoidant Attachment & 1.00 & 3.40 & 2.43 & .39 & -0.82 \\
Ambivalence-Worry (Ambivalent) Attachment & 1.00 & 3.67 & 2.31 & .57 & -0.11 \\
Ambivalence-Merger (Anxious) Attachment & 1.00 & 3.67 & 2.11 & .75 & -0.01 \\
\hline
\end{tabular}

Table 47 indicates that most EMTs use Secure Attachment a little $(M=3.34)$, while EMTs disagree a little that they use the remaining three attachment styles: Avoidant Attachment $(M=2.43)$; Ambivalence-Merger (Anxious) Attachment $(M=2.31)$; and Ambivalence-Worry (Ambivalent) Attachment $(M=2.11)$. Tables $48-51$ provide information on the item statistics per subscale. Table 48 indicates that EMTs find it difficult to get close to others (this was a reverse scored item, which indicates that the EMTs answered this question very low on the 
scale), but that being close to someone gives them a great sense of comfort $(M=3.56)$. Their greatest weaknesses are in worrying that their partner will not want to stay with them $(M=$ $2.04)$, and in their desire to be close to someone scaring others away $(M=2.00)$.

\section{Table 48}

Avoidance Attachment Item Statistics $(n=50)$

\begin{tabular}{lcc}
\hline \multicolumn{1}{c}{ Item } & $M$ & $S D$ \\
\hline I am very comfortable being close to others. & 2.80 & .93 \\
I find it easy to be close to others. & 2.54 & .93 \\
I prefer not to be too close to others. & 2.38 & 1.01 \\
Others want me to be more intimate than I feel comfortable being. & 2.24 & .98 \\
I get uncomfortable when someone wants to be very close. & 2.20 & .88 \\
\hline
\end{tabular}

\section{Table 49}

Ambivalence-Worry (Ambivalent) Attachment Item Statistics $(n=50)$

\begin{tabular}{lcc}
\hline \multicolumn{1}{c}{ Item } & $M$ & $S D$ \\
\hline I don't worry about others abandoning me. & 2.78 & 1.22 \\
I often worry that my partner doesn't really love me. & 2.12 & 1.08 \\
I often worry my partner will not want to stay with me & 2.04 & 1.14 \\
\hline
\end{tabular}

\section{Table 50}

Ambivalence-Merger (Anxious) Attachment Item Statistics $(n=50)$

\begin{tabular}{lcc}
\hline \multicolumn{1}{c}{ Item } & $M$ & $S D$ \\
\hline I have trouble getting others to be as close as I want them to be. & 2.22 & 1.04 \\
I find others are reluctant to get as close as I would like. & 2.12 & .82 \\
My desire to merge sometimes scares people away. & 2.00 & .81 \\
\hline
\end{tabular}

\section{Table 51}

Secure Attachment Item Statistics $(n=50)$

\begin{tabular}{lcc}
\hline \multicolumn{1}{c}{ Item } & $M$ & $S D$ \\
\hline When I'm close to someone, it gives me a sense of comfort about life in general & 3.56 & .58 \\
It feels relaxing and good to be close to someone. & 3.54 & .71 \\
Being close to someone gives me a source of strength for other activities. & 2.92 & .92 \\
\hline
\end{tabular}


Multivariate analysis of variance (MANOVA) was used to determine differences between EMT-B's and EMT-P's on a linear combination of the four attachment subscales. Table 52 provides group means and standard deviations for each of the four attachment subscales:

Table 52

Group Means and Standard Deviations for Attachment Subscale Results

\begin{tabular}{llccc}
\hline Attachment Subscale & Group & $M$ & $S D$ & $n$ \\
\hline Secure Attachment & EMT-B & 3.53 & .39 & 15 \\
& EMT-P & 3.27 & .70 & 33 \\
Ambivalence-Merger (Anxious) Attachment & EMT-B & 2.64 & .58 & 15 \\
& EMT-P & 1.83 & .67 & 33 \\
Avoidant Attachment & EMT-B & 2.53 & .22 & 15 \\
& EMT-P & 2.41 & .45 & 33 \\
Ambivalence-Worry (Ambivalent) Attachment & EMT-B & 2.53 & .47 & 15 \\
& EMT-P & 2.18 & .58 & 33 \\
\hline
\end{tabular}

Skewness statistics range from a low of -0.94 for Secure Attachment to a high of -0.01 for Ambivalence-Merger (Anxious) Attachment, suggesting that attachment styles may be deemed normally distributed. Equality of variance-covariance matrices assumption was not met (Box's $M=27.40, F(10,3572.52)=2.41, p=.007)$. Thus, Pillai's Trace, which “is very robust and not highly linked to assumptions about the normality of the distribution of the data" (oak.ucc.nau.edu, p. 2) was used (results shown below). Bartlett's test of sphericity suggests that there is sufficient inter-correlation among the dependent variables $\left(\chi^{2}=20.91, d f=6, p<.002\right)$. MANOVA indicates that there are significant differences between the two groups of EMTs (EMT-B's and EMT-P's) on a linear combination of the four attachment subscales (Pillai's trace $=0.30, F(4,43)=4.534 p=.004$, eta squared $=.30)$.

Univariate analysis indicated that EMT-B's have significantly $(p<.05)$ higher scores on Ambivalence-Worry (Ambivalence) Attachment $(M=2.53, S D=0.47)$ than EMT-P's $(M=2.18$, $S D=0.58)$. EMT-B scores were also significantly higher $(p<.001)$ on Ambivalence-Merger 
(Anxious Attachment) $(M=2.64, S D=0.58)$ than EMT-P scores $(M=1.83, S D=0.67)$. What this indicates is that EMT-B's appear to be both more ambivalently and more anxiously attached in their relationships than EMT-P's.

\section{Research Question Five}

Research question five sought to identify the nature of the relationship between PTSD severity and emotional intelligence, coping skills, resiliency, and attachment. Normality was checked through the use of P-P plots, which demonstrated the data points following closely along the normality line. Skewness statistics range from a low of -0.163 for Emotional Intelligence to a high of .67 for PTSD, suggesting that the variables may be deemed normally distributed. Homoscedasticity was evaluated through the use of scatterplots, with the results demonstrating a pattern of data points that were equally distributed both above and below zero on the $\mathrm{X}$-axis, as well as to the left and right of zero on the Y-axis. Therefore, both normality and homoscedasticity are established. Multicollinearity was evaluated by examining the VIF, with all VIF values below five indicating that this assumption has been met.

To identify the nature of the relationship between PTSD severity and emotional intelligence, coping skills, resiliency, and attachment, a direct entry Multiple Linear Regression Analysis was performed. Because separate analyses of EMT-B's and EMT-P's revealed no differences between EMT level with regard to emotional intelligence, coping, resilience, or attachment, there was no need to run separate analyses to identify differences by means of Multiple Linear Regression. Attachment was comprised of Avoidant Attachment, Ambivalent-Worry (Ambivalent) Attachment, Ambivalent-Merger (Anxious) Attachment, and Secure Attachment; Emotional Attachment was comprised of Perception of Emotion, Managing Own Emotions, Utilization of Emotion, and Managing Others' Emotions; Coping 
was comprised of Emotional Social Support, Problem Solving, Avoidance, and Use of Positive Thinking; and the final variable was Resiliency, resulting in thirteen independent variables.

Table 53 indicates the means, standard deviations, and the correlation coefficients in the full model, which includes all of the subscale items included in the regression analysis, and indicates the degree of correlation between PTSD and each of the independent variables/subscale items. The correlation coefficients demonstrated the strength of the relationship between PTSD and the independent variables. Positive coefficients demonstrated a positive relationship between the variable and PTSD, and a negative number indicates a negative relationship between the variable and PTSD.

Based on Table 53, it is clear that only four subscales did not have a significant correlation with PTSD (Use of Positive Thinking; Perception of Emotion; Resilience; and Secure Attachment). With regard to the remaining subscale items, the following results were indicated, in order of the strength of their correlation with PTSD: as Emotional Social Support increases, so do PTSD scores (.647); as Managing Others' Emotions (.597) scores increase, so do PTSD scores; Managing Own Emotions increases, so do PTSD scores (.544); as Perception of Emotion (.454) scores increase, so do PTSD scores; as Anxious Attachment increases, so do PTSD scores (.534); as Problem Solving increases, so do PTSD scores (.504); as Avoidance Coping (.414) increases, so do PTSD scores; as Ambivalent Attachment (.390) increases, so do PTSD scores and as Utilization of Emotion (.304) increases, so do PTSD scores, all of which demonstrated a positive correlation with PTSD. 


\section{Table 53}

Multiple Linear Regression Variable Mean, Standard Deviation, and Bivariate Correlation $(n=50)$

\begin{tabular}{|c|c|c|c|c|c|c|c|c|c|c|c|c|c|c|c|}
\hline Variable & M & SD & 2 & 3 & 4 & 5 & 6 & 7 & 8 & 9 & 10 & 11 & 12 & 13 & 14 \\
\hline 1PTSD Scores & 21.78 & 19.13 & .271 & .265 & .650 & .250 & -.274 & -.373 & -.212 & -.175 & -.297 & -.207 & -.019 & .250 & .390 \\
\hline 2Use of emotional social support & 2.02 & 0.66 & & .647 & .422 & .436 & -.056 & .107 & .317 & .407 & -.026 & .277 & .106 & .294 & .156 \\
\hline 3Problem solving & 2.09 & 0.91 & & & .504 & .415 & -.098 & .005 & .067 & .294 & -.092 & .174 & .202 & .382 & .235 \\
\hline 4Avoidance coping & 1.58 & 0.52 & & & & .414 & -.444 & -.486 & -.279 & -.234 & -.485 & -.161 & .146 & .643 & 395 \\
\hline 5Use of positive thinking & 2.29 & 0.63 & & & & & -.019 & -.092 & .042 & .282 & -.079 & .302 & .197 & .297 & .333 \\
\hline 6 Perception of emotion & 3.80 & 0.64 & & & & & & .454 & .506 & .305 & .494 & .153 & .044 & -.373 & -.367 \\
\hline 7Managing own emotion & 3.88 & 0.64 & & & & & & & .544 & .568 & .633 & .360 & .069 & -.410 & -.399 \\
\hline 8Managing others' emotion & 3.97 & 0.53 & & & & & & & & .597 & .353 & .278 & -.179 & -.299 & -.190 \\
\hline 9Utilization of emotion & 3.69 & 0.65 & & & & & & & & & .304 & .636 & .165 & -.260 & -.117 \\
\hline 10Resilience Scale & 31.58 & 6.87 & & & & & & & & & & .068 & .065 & -.317 & -.358 \\
\hline 11Attachment: Security & 3.34 & 0.63 & & & & & & & & & & & .109 & -.101 & .041 \\
\hline 12Attachment: Avoidance & 2.43 & 0.39 & & & & & & & & & & & & .256 & .136 \\
\hline 13Attachment: Ambivalence-worry & 2.31 & 0.57 & & & & & & & & & & & & & .534 \\
\hline 14Attachment: Ambivalence-merger & 2.11 & 0.75 & & & & & & & & & & & & & \\
\hline
\end{tabular}

A direct entry Multiple Linear Regression Analysis was performed using all thirteen of the independent variables to determine which, if any were predictors of PTSD. This overall model with all thirteen of the independent variables entered produced an $R^{2}$ of 0.588 for the model, indicating that approximately $59 \%$ of the variation in PTSD symptom expression could be explained by this model. However, because this was a small sample size, the adjusted $R^{2}$ was used. The adjusted $R^{2}$ of .439 indicated that 
approximately $44 \%$ of the variation in PTSD symptoms could be explained by this Model. The ANOVA Table indicated that the model predicted PTSD symptom expression to a statistically significant degree, with the model being significant at $p=$ .001 , and suggesting a linear relationship. From this, the following significance was shown: Model $1=R^{2}=.588, R_{\text {adj }}^{2}=.439, F(13,36)=3.95, p<.001$. The full regression analysis consisting of 13 predictors as a set resulted in three subscale items that were significantly correlated with PTSD: Avoidance Coping $(\beta=.842, p<.001)$;

Ambivalence-Worry (Ambivalent) Attachment $(\beta=-.411, p=.017$ ); and AmbivalenceMerger (Anxious) Attachment $(\beta=.344, p=.018)$, explaining approximately $44 \%$ of the variance. The Regression Analysis Results are shown in Table 54.

\section{Table 54}

Regression Analysis Results - Full Model

\begin{tabular}{lrllrl}
\hline Variable/Subscale Item & $b$ & SE & \multicolumn{1}{c}{$\beta$} & \multicolumn{1}{c}{$t$} & \multicolumn{1}{c}{ Sig. } \\
\hline Perception of Emotion & .011 & .012 & .152 & .954 & .346 \\
Managing Own Emotions & -.001 & .016 & -.014 & -.075 & .941 \\
Managing Others' Emotions & -.025 & .023 & -.216 & -1.082 & .286 \\
Utilization of Emotion & .013 & .024 & .104 & .536 & .595 \\
Emotional Social Support & .016 & .017 & .149 & .902 & .373 \\
Problem Solving & -.015 & .021 & -.111 & -.700 & .489 \\
Avoidance Coping & .079 & .018 & .842 & 4.322 & .000 \\
Use of Positive Thinking & -.006 & .018 & -.044 & -.311 & .758 \\
Resiliency & .009 & .011 & .125 & .799 & .429 \\
Avoidant Attachment & -.027 & .031 & -.109 & -.868 & .391 \\
Ambivalence-Worry (Ambivalent) & -.118 & .047 & -.411 & -2.505 & .017 \\
Attachment & & & & & \\
Ambivalence-Merger (Anxious) Attachment & .074 & .030 & .344 & 2.470 & .018 \\
Secure Attachment & -.041 & .039 & -.162 & -1.069 & .292 \\
(Constant) & .001 & .775 & & & \\
\hline$R^{2}$
\end{tabular}

$R^{2}=.588$, Adj. $R^{2}=0.439, F_{(13,36)}=3.95, p<0.001$

A restricted model was run using only the significant predictors (Avoidance

Coping, Ambivalence-Worry (Ambivalent) Attachment, and Ambivalence-Merger

(Anxious) Attachment). The Model Summary with the three significant variables entered 
produced an $R^{2}$ of 0.534 for the model, indicating that approximately $53 \%$ of the variation in PTSD symptom expression could be explained by this model. However, because this was a small sample size, the adjusted $R^{2}$ was used once again. The adjusted $R^{2}$ of .503 indicated that approximately $50 \%$ of the variation in PTSD symptoms could be explained by this Model. The ANOVA Table indicated that this model predicted PTSD symptom expression to a statistically significant degree, with the models being significant at $p<.001$.

From this, the following significance was shown: Model $1=R^{2}=.534, R^{2}{ }_{\text {adj }}=$ $.503, F(3,46)=17.56, p<.001$. The Regression Analysis Results Table (Table 55) once again confirms the statistical significance of Avoidance Coping $(\beta=.807, p<.001)$; Ambivalence-Worry (Ambivalent) Attachment $(\beta=-.429, p=.004)$; and AmbivalenceMerger (Anxious) Attachment $(\beta=.300, p=.015)$, explaining approximately $50 \%$ of the variance.

\section{Table 55}

Regression Analysis Results - Restricted Model

\begin{tabular}{|c|c|c|c|c|c|}
\hline Variable/Subscale Item & $b$ & $\mathrm{SE}$ & $\beta$ & $t$ & Sig. \\
\hline Avoidance Coping & .076 & .012 & .807 & 6.120 & .000 \\
\hline Ambivalence-Worry (Ambivalent) & -.123 & .041 & -.429 & -2.994 & .004 \\
\hline Attachment & & & & & \\
\hline $\begin{array}{l}\text { Ambivalence-Merger (Anxious) Attachment } \\
\text { (Constant) }\end{array}$ & $\begin{array}{l}.065 \\
-.395\end{array}$ & $\begin{array}{l}.026 \\
.210\end{array}$ & .300 & 2.515 & .015 \\
\hline
\end{tabular}

These results indicate that there is a significant positive correlation between EMTs who use Avoidance Coping and higher PTSD scores; that there is a significant negative correlation between EMTs who are Ambivalently Attached and PTSD scores; 
and that there is a significant positive correlation between EMTs who are Anxiously Attached, and PTSD scores.

\section{Summary of the Findings}

\section{Research Question One}

Approximately a third $(n=15,30 \%)$ of the EMTs were assumed to have PTSD based on PCL-5 total symptom criteria, 36\% based on DSM-5 endorsed items criteria.

\section{Research Question Two}

No significant differences between the scores for EMT'B's and EMT-P's based on total PCL-5 symptom scores $\left(\chi^{2}=1.37, \mathrm{df}=1, p=.35\right)$ or DSM-5 criterion scores $\left(\chi^{2}\right.$ $=0.44, \mathrm{df}=1, p=.51)$.

\section{Research Question Three}

EMTs use Positive Thinking $(M=2.29)$, Problem Solving $(M=2.09)$, Emotional Social Support $(M=2.02)$, and Avoidance Coping $(M=1.58)$, respectively, to cope with stressful situations. These scale means indicate that they use these coping strategies only a "little bit." No significant differences between EMT-B's and EMT-P's on the use of the four coping strategies. No significant differences between EMT-B's and EMT-P's on the four coping strategies (Wilk's lambda $=0.83, F(4,43)=2.20, p=.085$, eta squared $=$ $.17)$.

\section{Research Question Four}

\section{Emotional intelligence}

The greatest percentage of EMTs $(n=29 ; 58 \%)$ fell into the average range on emotional intelligence. However, approximately $30 \%(n=15)$ of EMTs scored in the unusually high range on emotional intelligence. EMTs demonstrated the greatest 
strength in their ability to manage theirs and others' emotions (Managing (their) Own Emotions: $M=3.88$; Managing Others' Emotions: $M=3.85)$, with the ability to perceive emotions falling very closely behind (Perception of Emotion: $M=3.80$ ). Their weakest area was in their ability to utilize emotions (Utilization of Emotion: $M=3.69$ ). No significant differences between EMT-B's and EMT-P's on the four emotional intelligence subscales (Wilk's lambda $=0.96, F(4,43)=0.47, p=.757$, eta squared $=$ $.04)$.

\section{Resilience}

The mean total resilience score is $3.16(\mathrm{SD}=0.68)$, indicating that it is "often true" that the EMTs in this study are resilient. Thirty-Eight percent of EMTs fell into the highest twenty-five percent of the population, or the most resilient of the population, and $38 \%$ fell into the lowest $25 \%$ of the population, or the least resilient of the population. EMT-B's were nearly one-and-three-fourths times less resilient than EMT-P's. The proportion of EMT-B's and EMT-P's did not differ on any of the categories of Resiliency $\left(\chi^{2}=5.95, d f=3, p=.114\right)$.

\section{Attachment}

EMTs use Secure Attachment a little $(M=3.34)$, while EMTs disagree a little that they use the remaining three attachment styles: Avoidant Attachment $(M=2.43)$; Ambivalence-Merger (Anxious) Attachment $(M=2.31)$; and Ambivalence-Worry (Ambivalent) Attachment $(M=2.11)$. There were significant differences between the two groups of EMTs (EMT-B's and EMT-P's) on the four attachment subscales (Pillai's trace $=0.30, F(4,43)=4.534 p=.004$, eta squared $=.30)$. Univariate analysis indicated that EMT-B's have significantly $(p<.05)$ higher scores on Ambivalence-Worry 
(Ambivalence) Attachment $(M=2.53, S D=0.47)$ than EMT-P's $(M=2.18, S D=0.58)$.

EMT-B scores were also significantly higher $(p<.001)$ on Ambivalence-Merger

(Anxious Attachment) $(M=2.64, S D=0.58)$ than EMT-P scores $(M=1.83, S D=0.67)$.

\section{Research Question Five}

The bivariate correlation matrix noted that only four subscales did not have a significant correlation with PTSD (Use of Positive Thinking; Perception of Emotion; Resilience; and Secure Attachment). With regard to the remaining subscale items, the following results were indicated, in order of the strength of their correlation with PTSD: as Emotional Social Support increases, so do PTSD scores (.647); as Managing Others' Emotions (.597) scores increase, so do PTSD scores; Managing Own Emotions increases, so do PTSD scores (.544); as Perception of Emotion (.454) scores increase, so do PTSD scores; as Anxious Attachment increases, so do PTSD scores (.534); as Problem Solving increases, so do PTSD scores (.504); as Avoidance Coping (.414) increases, so do PTSD scores; as Ambivalent Attachment (.390) increases, so do PTSD scores; and as Utilization of Emotion (.304) increases, so do PTSD scores, all of which demonstrated a positive correlation with PTSD.

Three subscales were significantly correlated with PTSD: Avoidance Coping; Ambivalence-Worry (Ambivalent) Attachment; and Ambivalence-Merger (Anxious) Attachment The model predicted PTSD symptom expression to a statistically significant degree, with the model being significant at $p<.001\left(R^{2}=.534, R_{\text {adj }}^{2}=.503, F(3,46)=\right.$ 17.56). The Regression Analysis Results Table confirmed the statistical significance of Avoidance Coping $(\beta=.807, p<.001)$; Ambivalence-Worry (Ambivalent) Attachment $(\beta=-.429, p=.004) ;$ and Ambivalence-Merger (Anxious) Attachment $(\beta=.300, p=$ 
.015), explaining approximately $50 \%$ of the variance. These results indicated that EMTs with higher PTSD scores are more likely to be those with higher scores in Avoidance Coping and Ambivalence Merger (Anxious) Ambivalence, but lower scores in Ambivalence-Worry (Ambivalent) Attachment scores. Of the three, the most important predictor was Avoidance Coping $(\beta=.81)$.

\section{Integration of Quantitative and Qualitative Results}

It is important to consider the agreement and disagreement between the quantitative and the qualitative research results, and to identify whether there were themes that arose in the qualitative data that were not present in the quantitative results. These will be presented in this section, after which a full discussion of the findings will be provided in Chapter 5 .

\section{Areas of Agreement}

Both the quantitative and the qualitative results agree on the difficult working conditions for EMTs, and the presence of PTSD due to the exposure to traumatic events. While examining the forthcoming themes from the qualitative portion of the study, the final themes of Resiliency and Contributors to PTSD were also noted areas of agreement between the two portions of the study.

Other areas of agreement include all four of the independent variables: Coping; Emotional Intelligence; Attachment; and Resiliency, with each revealing common ground with relation to both public and private sector EMTs, indicating similar work-related conditions that factor into PTSD expression in EMTs, which also speak to the type of coping, attachment, and resiliency that is demonstrated in this sector of EMTs in their ability to continue on despite such challenging work conditions. 


\section{Areas of Disagreement}

While both the quantitative and qualitative results agree on the difficult working conditions for EMTs in general, the fact that because they are privately funded, the private sector does not allow for them to have either the quarters to go to after traumatic calls as the fire service provides so that they can just take some time to unwind or destress or to "shut off" as one EMT expressed, but that they have to sit in the ambulance for the entirety of their shift, nor do they have the opportunity to debrief with "chaplains and other resources to assist them in dealing with traumatic calls" as the EMTs with the public sector are afforded. Additionally, because the private sector is privately funded, it results in the need for the EMTs to run calls as often as possible, with the EMTs expressing that they ran calls on a back-to-back basis without any break in shift for breaks, meals, or even to use the restroom.

With regard to the four independent variables, while both private and public sector EMTs indicate similar work-related conditions, because of the perceived differences between the private and the public sector of the field, private sector EMTs possibly demonstrate greater levels of resilience, coping, emotional intelligence, and attachment with regard to the ability to continue on despite these perceived differences.

\section{New Themes Identified in the Qualitative Data}

A theme identified in the qualitative data that was not present in the quantitative data was the theme of Work Quality, or Altruism. This theme consisted of the element of enjoyment for their work, and of obtaining satisfaction from helping others. It also included placing the value of helping someone else over work that would provide greater pay or recognition. 
Another theme that arose was the theme of Disenfranchisement, which included the sub-category of Societal Acknowledgement. This theme was seen in multiple areas, such as the discrepancy in pay and lack of retirement for the same work, limited to no retirement benefits, and less updated and appropriate resources such as equipment.

The lack of societal acknowledgement could be seen in areas such as the "public sector EMTs [being] seen as "hero's" despite the fact that they did less, ran fewer calls, and often never showed up on scene;" and the lack of recognition by both the public sector ("In the private sector, EMS is [considered to be] beneath fire") and the public; and work setting and lack of the same resources as the public sector as described above, as well as outdated necessary equipment ("Public is funded and gets updated equipment, where private is not, and it all depends on getting your billing paid whether you had the money for upgrading equipment or even pay raises"). All three of the participants for the qualitative portion described not being taken seriously as private sector EMTs, with the public seeing them as "ambulance drivers" instead of EMS personnel with expertise and skills, and they reported not being acknowledged by the public as being present at major traumatic events and not being given any credit for the work that they often did hand-inhand with the public sector EMTs.

\section{Summary}

The qualitative portion of this study utilizing individual interviews with private sector EMTs revealed 5 overarching themes. These were: work environment, work quality/altruism, disenfranchisement, resiliency, and contributors to PTSD, the subcategory of altruism under the work quality/altruism category, and the sub-category of societal acknowledgment under the disenfranchisement category. 
The quantitative portion of this study found that $30 \%$ of the EMTs in this study overall met criteria for PTSD in accordance to the PCL-5 cutoff criteria and $36 \%$ in accordance to the DSM-5 cutoff criteria, and that $21 \%$ of the EMT-B's and $40 \%$ of the EMT-P's met the PCL-5 criterion for PTSD and 40\% of the EMT-B's and 30\% of the EMT-P's met the DSM-5 diagnostic criteria for PTSD. With only two EMT-I's, there was not a great enough sample size for them to be a representative sample, and they were excluded from the analysis. Results showed no difference in the severity in which EMTB's and EMT-P's experience PTSD, indicating that both EMT-B's and EMT-P's experience PTSD in a similar manner. Both EMT-B's and EMT-P's use Positive Thinking as their primary method of coping, with no significant differences found between EMT-B's and EMT-P's in each of the four identified coping styles. Fifty-eight percent of EMTs fell into the average range on a measure of emotional intelligence, and another $30 \%$ falling into the unusually high range on the same measure of emotional intelligence. The EMTs in this study showed the greatest struggle in being able to utilize their own emotions, while they found it much easier to manage their own and others' emotions and in the ability to perceive emotions. The EMTs fell into both the highest $25 \%$ of the population and into the lowest $25 \%$ of the population $(38 \%)$ on a measure of resiliency (38\%), and EMT-B's were shown to be nearly one-and-three-fourths times less resilient than EMT-P's. The greatest percentage of the EMTs in this study were found to be securely attached a little bit, and EMT-B's were found to have significantly both more ambivalent attachment and significantly more anxious attachment. Linear regression analysis results indicated that the most significant factors predicting PTSD were the use of avoidance coping and having ambivalent and anxious attachment styles, and that 
EMTs with higher PTSD scores were more likely to be those with higher scores in Avoidance Coping and Ambivalence Merger (Anxious) Ambivalence, but lower scores in Ambivalence-Worry (Ambivalent) Attachment scores. Of the three, the most important predictor was Avoidance Coping. 


\section{CHAPTER 5}

\section{SUMMARY, DISCUSSION, AND RECOMMENDATIONS \\ Introduction}

This final chapter of the dissertation will serve as a summarization of the previous four chapters, with the purpose of the study described, a review of the literature review presented, a description of both the methodology and the findings of the study, and a discussion of the applicability of those findings. Finally, the chapter will conclude by discussing the implications of the study for future practice clinically and with regard to further research opportunities.

\section{Summary of the Data}

The summary of the data is described below with the purpose of the study presented first, followed by a brief review of the literature on PTSD, and with the methodology of the study completing the summary portion of this chapter.

\section{Statement of the Problem}

Private sector EMTs are among the population of first responders on scene of often traumatic and horrific scenes, along with firefighters and police officers, exposing them to traumatic events as severe as other first responders. However, news agencies and the public do not recognize private sector EMTs. A lack of research has left a void in understanding how private sector EMTs experience PTSD, and to examine what unique risk factors and needs may be present.

\section{Purpose of the Study}

The purpose of this study was to examine the relationship between coping skills, emotional intelligence, attachment, and resilience on PTSD symptoms in private sector 
Emergency Medical Technicians (EMTs) workers, as well as to identify factors unique to this population of first responders.

\section{Literature Review Summary}

Posttraumatic Stress Disorder (PTSD) has a long history of research backing it, with initial research being performed on military populations, and on military veterans. It was initially believed that only military individuals could experience PTSD, but as research has continued in the field, it has become clear that anyone who has been exposed to a traumatic event or who has become aware of someone who has, can experience PTSD.

\section{Impact of Post-Traumatic Stress Disorder}

The effect of PTSD has been well documented, with PTSD being the most commonly diagnosed (Green \& Kaltman, 2003) disorder resulting from exposure to trauma (Matusko, Kemp, Paterson, \& Bryant, 2009).

\section{Psychological Factors in Post-Traumatic Stress Disorder}

\section{Coping Style}

Avoidant coping has been found to have a direct correlation with PTSD symptom severity has remained robust, which has been shown in a 2012 study on refugees that had settled in the Netherlands following war, persecution, and violence, with the study outcomes demonstrating a link between PTSD severity and avoidant coping (Huijts, Kleijn, can Emmerik, Noordhof, \& Smith, 2012); in a study on Israeli veterans who had been diagnosed with PTSD (Galor, \& Hentschel, 2012); and in a study published in 2012 on veterans who had been exposed to traumatic situations (Hassija, Luterek, NaragonGainey, Moore, \& Simpson, 2012). Avoidant coping was the only resulting factor that 
demonstrated a significant association with changes in PTSD symptom severity over a 1year period among veterans who had served in combat zones (Lee, Possemato, \& Ouimette, 2017). Finally, when examining the ability to adjust following a traumatic event, Slanbekova, Chung, Abildina, Sabirova, Kapbasova, and Karipbaev (2017) identified emotion-focused coping and the inability to manage one's emotions to be correlated with difficulties in adjustment, whereas the use of problem-focused coping and being able to manage one's emotions were correlated with greater symptom severity in couples following a divorce.

\section{Emotional Intelligence}

Emotional intelligence has been studied in multiple populations (Ghazali, 2014; Bodzy, Barreto, Swenson, Liguori, \& Costea, 2016; Stokes \& Jackson, 2014; Smith, Turner, Pati, Petrides, Sevdalis, \& Green, 2012; Gaher, Hofman, Simons, \& Hunsaker, 2013; Chantrell, 2009; O’Cleirigh, Ironson, Fletcher, \& Schneiderman, 2008;

Slanbekova, Chung, Abildina, Sabirova, Kapbasova, \& Karipbaev, 2017; Benoit, Bouthillier, Moss, Rousseau, \& Brunet, 2010). In each of the above listed research studies, it was proposed that individuals with greater levels of emotional intelligence would demonstrate lower levels of PTSD symptom severity. This was found to be the case in each of the studies, without exception.

\section{Attachment}

Individuals with attachment avoidance and attachment anxiety demonstrated greater levels of PTSD in a study by Busuito, Huth-Bocks, and Puro (2014) as well, with results confirmed in a meta-analysis performed by Woodhouse, Ayers, and Field (2015), as well in multiple other studies with various populations demonstrate the same 
connection between anxious and insecure attachment and PTSD symptomatology (Besser, Neria, \& Haynes, 2009; Holt, Jensen, Hansen, Elklit, \& Mogensen, 2016; Andersen, Elklit, \& Vase, 2011; Nye, Katzman, Bell, Kilpatrick, Brainard, \& Haaland, 2008; Arikan, Stopa, Carnelley, \& Karl, 2016; Avi, \& Yuval, 2010; Bogaerts, Daalder, Van Der Knaap, Kunst, \& Buschman, 2009; La Flair, Bradshaw, Mendelson, \& Campbell, 2015; Turunen, Haravouri, Punamaki, Suomalainen, \& Marttunen, 2014).

\section{Resilience}

Resilience has been associated with post-traumatic growth (Vera, 2013; OginskaBulik and Kobylarczyk, 2014), with positive emotions (Frederickson, 1998;

Frederickson, 2001), greater levels of physical wellness (Chang \& Taormina, 2011), higher levels of satisfaction with personal relationships, medical care, and community life (Chang \& Taormina, 2011), and lower levels of burnout (Chang \& Taormina, 2011). It has also been found to be a contributing factor in the determination of perceived levels of stress (Mroz, 2015), and it has been found to serve as a buffer against depressive symptoms (Sinclair \& Wallston, 2004).

\section{Vulnerability to Developing PTSD}

Avoidance factors can often be shown to mediate trauma and PTSD, with indications that one, preceding the other, may be a predictor. Specifically, the greater the level of experiential avoidance and post-traumatic exposure, the more likely individuals were to develop PTSD (Maack, Tull, \& Gratz 2012). The role of avoidance has been implicated in not only mediating between exposure and development, but it has also been implicated as a factor in maintaining PTSD symptoms (Maack, Tull, \& Gratz 2012). 


\section{Methodology}

\section{Qualitative Research Design}

A qualitative design was implemented utilizing individual interviews in phenomenology in order to understand the experiences of the participants.

\section{Quantitative Research Design}

This investigation utilized survey research followed by a correlational analysis of the results for the quantitative portion of this study. Online surveys was used using a quantitative approach to obtain the data, with questionnaires serving as the means by which the information was gathered. A non-experimental research design was chosen over an experimental design, as the goal of this research was to identify the relationships between the variables, rather than to utilize a specified treatment and measurement approach.

\section{Description of the Participants}

This sample of 50 participants for the quantitative portion of the study and three participants for the qualitative portion of the study consisted of private sector EMT-B's and EMT-P's from across the United States. The demographics for the qualitative portion of the study were as follows: $0 \%$ were female $(n=0)$ and $100 \%$ were male $(n=$

3). The participants ranged in age from 43 to 56 years of age, with a mean of 47.33 years of age and a standard deviation of 7.51. Breakdown of EMT levels included EMTBasic's $(33.3 \%, n=1)$ and EMT-Paramedic's $(66.7 \%, n=2)$. Of the 50 eligible participants for the quantitative portion of the study, $46 \%$ were female $(n=23)$ and $54 \%$ were male $(n=27)$. The participants ranged in age from 20 to 67 years of age, with a mean of 44.44 years of age and a standard deviation of 13.36. Breakdown of EMT levels 
included EMT-Basic's $(30 \%, n=15)$, EMT-Intermediate's $(4 \%, n=2)$, and EMT-

Paramedic's $(66 \%, n=33)$.

\section{Variables}

The variables used in this study were PTSD as the dependent variable, and coping skills, emotional intelligence, attachment, and resiliency as the four independent variables.

\section{Instruments}

\section{Post-Traumatic Stress Disorder}

The dependent variable in this study was PTSD. The instrument that was used to identify respondent match with PTSD symptoms was the PTSD Checklist (PCL-5), which was created to evaluate the presence and severity of PTSD symptoms as defined in the DSM-5.

\section{Coping}

The first independent variable in this study, Coping, was evaluated for through the use of The Brief COPE, a shortened version of the COPE, which identified 14 coping styles: Self-distraction, Active coping, Denial, Substance use, Use of emotional support, Use of instrumental support, Behavioral disengagement, Venting, Positive reframing, Planning, Humor, Acceptance, Religion, and Self-blame that fall into the four subscales Use of Emotional Social Support; Use of Problem Solving; Avoidance Coping, and Use of Positive Thinking.

\section{Emotional intelligence}

The second independent variable, Emotional intelligence, was evaluated for using The Schutte Emotional Intelligence Scale (SEIS), which is also known as the Schutte 
Self-Report Emotional Intelligence Test (SSEIT) and the Self-Report Emotional

Intelligence Test (SREIT), which indicated the respondent's emotional intelligence score:

Unusually Low, Normative/Average, or Unusually High.

\section{Attachment}

The third independent variable, Attachment, was evaluated for through the use of The Measure of Attachment Qualities (MAQ), which was designed to measure four subscales of attachment: Avoidant Attachment, Anxious (Ambivalent-Merger) Attachment, Ambivalent (Ambivalent-Worry) Attachment, and Secure Attachment.

\section{Resilience}

The final independent variable, Resilience, was evaluated for using the 10 item CD-RISC 10. The Connor Davidson - Resiliency Scale (CD-RISC) was created by as a means to measure resiliency.

\section{Procedure}

This study examined the level of PTSD symptoms experienced by EMTs and how coping techniques, emotional intelligence, resiliency, and attachment play a role in impacting these symptoms. The data was analyzed using both SPSS and qualitative evaluation techniques, with content analysis being the primary evaluation method. To identify specific experiences and needs of this population, individual interviews and analysis was utilized using a qualitative approach to the data. To identify the unique contribution of each of the variables (coping techniques, emotional intelligence, resiliency, and attachment) in the explanation of PTSD symptomatology in EMTs, a Multiple Linear Regression analysis was performed. 


\section{Qualitative Data}

The participant interviews were transcribed and then evaluated by open, line-byline coding, followed by axial coding. Memo writing was also performed on the transcripts for further evaluation, and to identify any additional themes that may have been missed previously. Codes were then identified, and single participant categories were retained that had been identified among individual participants. Overlapping categories were then identified among all three of the participants, with these codes collapsed in order to identify the most common areas and themes amongst the participants as a whole. This researcher continued to return to the data throughout the analysis process in order to ensure soundness between the data as it was identified and each of the research questions, after which the memos that had been written previously during the coding of the data were revisited in order to 1) identify the themes and commonalities among the participants; 2) to assist in the connection of the categories to each other; and 3) to identify the uniqueness's of each of the participants. The purpose behind this was to identify any differing populations that could be an additional area of focus in future studies on private sector EMTs, as well as to identify service needs as specific to this population, and to identify service needs of any additional but similar populations.

\section{Quantitative Data}

To evaluate for and analyze the quantitative data, several analysis measures were used. To evaluate for Research Question One, the data was evaluated through the identification of the presence of PTSD as per the cutoff score on the PCL-5, as well as through the grouping of items into clusters in accordance with the diagnostic criteria in 
the DSM 5. Frequencies were then run to identify the presence of PTSD in this sample of private sector EMTs.

For Research Question Two, diagnostics were run to breakdown the EMTs in this study into their EMT status (EMT-B or EMT-P) to identify the presence of PTSD.

Crosstabs were then run to identify the specific items endorsed per cluster and per EMT level. An Independent Sample T-Test was run using the sample of EMT-B's and EMTP's to identify any differences between the scores for the two groups.

For Research Question Three, Coping was grouped into the 14 subscales of the Brief COPE to provide the reader with an understanding of which items comprised which of the subscales, after which the subscales were grouped into the four coping styles as identified by the authors. A MANOVA was run to identify the coping styles used by EMT level.

For Research Question Four, the scoring breakdown of the SEIS was shown along with the breakdown of the items comprising each of the four subscales of the measure. From there, frequencies were run to identify the levels of Emotional Intelligence for the EMTs in this study. The scoring breakdown was presented for the CD-RISC, with frequencies run to identify the levels of resiliency for the EMTs in this study. To evaluate for Attachment, the subscales were presented with each of the items comprising those subscales, after which frequencies were run to identify the attachment styles and their levels utilized by the private sector EMTs in this study.

For Research Question Five, a Multiple Linear Regression was run in order to identify the nature of the relationship between PTSD severity and Emotional Intelligence, Coping Skills, Resiliency, and Attachment. 


\section{Discussion}

\section{Qualitative Results}

The individual interviews with private sector EMTs revealed 5 overarching themes. These were: work environment, work quality/altruism, disenfranchisement, resiliency, and contributors to PTSD, the sub-category of altruism under the work quality/altruism category, and the sub-category of societal acknowledgment under the disenfranchisement category. These themes were a result of their decisions to work in the private versus the public sector of the field, their approach to the job even when in the face of difficult circumstances, and despite being disenfranchised in their work. Each of these are tied into and expounded on in the quantitative portion of the discussion, below.

\section{Quantitative Results}

\section{Research Question One}

This study found that $30 \%$ of the EMTs in this study overall met criteria for PTSD in accordance to the PCL-5 cutoff criteria and 36\% in accordance to the DSM-5 cutoff criteria, and that $21 \%$ of the EMT-B's and $40 \%$ of the EMT-P's met the PCL-5 criterion for PTSD, and 40\% of the EMT-B's and 30\% of the EMT-P's met the DSM-5 diagnostic criteria for PTSD. With only two EMT-I's, there was not a great enough sample size for them to be a representative sample, and they were excluded from the analysis.

The above results indicate that private sector EMTs experience exposure to traumatic events in the course of their work that result in intrusion symptoms; avoidance of any memories, thoughts, feelings, or external reminders of the event; and negative alterations in their emotions, and alternations in both arousal and reactivity. This is likely 
due to the extreme nature of their job in which they routinely respond to medical emergency calls that can include calls of a very serious nature, or calls in which there is severe physical trauma to the body.

This is consistent with the literature on exposure to traumatic events as seen in the work of multiple researchers (Beckman, 2015; Dass-Brailsford, 2010; Stellman, Smith, Katz, Sharma, Charney, Herbert, Southwick, 2008; Long, Meyer, \& Jacobs, 2007; Smith, 2007; VanDeusen \& Way, 2006; Simons, Gaher, Jacobs, Meyer, \& Johnson-Jimenez, 2005; McCaslin, Jacobs, Meyer, Johnson-Jiminez, Metzler, \& Marmar, 2005; Fullerton, Ursano, \& Wang, 2004), with several researchers noting that PTSD is the most commonly diagnosed (Green \& Kaltman, 2003) disorder resulting from exposure to trauma (Matusko, Kemp, Paterson, \& Bryant, 2009).

A study on paramedic trainees (Fjeldheim, Nöthling, Pretorius, Basson, Ganasen. Heneke Cloete, and Seedat, 2014) found that $16 \%$ of these trainees met criteria for PTSD. Additionally, Rinker (2015) found that a meta-analysis by Meyer, Zimering, Daly, Knight, Kamholz, and Gulliver (2012) identified that prior to 2000, anywhere between $18 \%$ and $37 \%$ of career firefighters met the criteria for PTSD. However, more recent studies (Del Ben, Scotti, Chen, and Fortson, 2006; Meyer et al., 2012) note that rate to be between four and eight percent for firefighters and EMTs. Therefore, the 30\% (according to PCL-5 criteria) and 36\% (according to DSM-5 criteria) of EMTs in this study fall on the high side of those meeting criteria for PTSD in comparison to other first responders in the field.

The work environment for private sector EMTs is such that they are exposed to traumatic situations routinely, but that, because they are privately funded, does not allow 
for them to have either the quarters to go to after traumatic calls as the fire service provides so that they can just take some time to unwind or de-stress or to "shut off" as one EMT expressed, but that they have to sit in the ambulance for the entirety of their shift, nor do they have the opportunity to debrief with "chaplains and other resources to assist them in dealing with traumatic calls" as the EMTs with the public sector are afforded. The inability to have down time following traumatic calls by having quarters to return to in order to take a break or to destress, the work environment in which they are forced to run one call after another without any type of a pause despite the type of call to which they have responded, as well as the lack of a supportive work environment in which they are provided chaplains or stress debriefing is likely to have an impact on their ability to deal with the traumatic event to which they have been exposed, and is likely to result in PTSD symptoms. Marchand, Boyer, Nadeau, Beaulieu-Prévost, \& Martin (2015) noted specifically that post-traumatic factors that may increase the likelihood of developing PTSD may include factors such as short periods of time allocated by others to recover from the event, dissatisfaction with one's support system, or the lack of social support outside of the incident-related situation, all of which the EMTs in this study expressed experiencing in their work situations.

\section{Research Question Two}

The results also showed no difference in the severity in which EMT-B's and EMT-P's experience PTSD, indicating that both EMT-B's and EMT-P's experience PTSD in a similar manner. While EMT-B's and EMT-P's have different scopes of practice, both are exposed to the same events since they are both on the same unit and they are both responding to the same calls; therefore, despite the difference between the 
EMT levels with regard to the care provided by each, the factors that result in PTSD remain the same for both. It is likely for this reason that the results demonstrated no differences in the severity in which EMT-B's and EMT-P's experience PTSD. These results are the first of its kind as known to this researcher, examining differences between EMT levels. Therefore, these results provide new information to the field and expanding the knowledge on EMT levels, and allow for an area of further exploration by future researchers.

\section{Research Question Three}

Both EMT-B's and EMT-P's use Positive Thinking as their primary method of coping, with no significant differences found between EMT-B's and EMT-P's in each of the four identified coping styles. It is probable that the use of positive thinking is due to the nature of the work in the respect that there is the expectation that the treatment provided by the EMTs will assist those they are treating to benefit them or to assist in taking care of the medical problem until they can get the patient to the hospital for further treatment. It is also a possibility that "seeing things in a different light" and "looking for something good in what is happening" assists the EMTs in continuing on with their difficult jobs, as well as in finding the blessings in their lives that they may have missed had they not had the opportunity to see life differently from the perspective of their work.

The results of this study are in contradiction to results found in police officers who experienced PTSD, with their primary coping style being emotionally focused coping strategies (Plaxton-Hennings, 2004); and with research by Regehr, Goldberg, and Hughes (2002, as cited in Avraham, Glodblatt, \& Yafe, 2014), who found that EMTs maintained a sort of emotional distance while treating their patients. Halpern, Gurevich, 
Schwartz, and Brazeau (2009, as cited in Avraham, Glodblatt, \& Yafe, 2014), found that EMTs avoided thinking about the current situation and kept their thoughts about the situation to themselves. Ogińska-Bulik and Kobylarczyk (2014) identified positive reframing as one of the coping skills that paramedics used; however, it was not the primary method of coping, but followed active coping, planning, and acceptance. Morrison (2011) also found positive reappraisal as a coping technique for EMTs, but again, not the primary method of coping.

Therefore, it the EMTs in this study appear to utilize coping differently than other first responders. It is possible that there may be a qualitative difference between the field experience of EMTs and other first responders, with private sector EMTs. The fact that the EMTs reported a great deal of altruism in their work, it may be that the reasons drawing them to their work is different from those in the public sector because the perks that are available to those in the public sector are not available to those in the private sector, thereby drawing those in the private sector to the field for greater interpersonal reasons, rather than for the perks that the public sector affords. This may lead the private sector to view their work in a more positive manner, which is possibly the reason that positive thinking is their primary method of coping. Halpern, Gurevich, Schwartz, and Brazeau (2009, as cited in Avraham, Glodblatt, \& Yafe, 2014) concur that there is not enough research on ambulance personnel, although they do not make a distinction between the public and private sector of providers. 


\section{Research Question Four}

\section{Emotional intelligence}

Fifty-eight percent of EMTs fell into the average range on a measure of emotional intelligence, and another $30 \%$ falling into the unusually high range on the same measure of emotional intelligence. The sole study found on EMTs and emotional intelligence levels was Rinker's (2015) study on PTSD in firefighters and emergency medical personnel, which documented emotional intelligence scores falling into the high range. In comparison, with over $50 \%$ of the EMTs in this study falling into the average range, this leaves them falling below the range of those examined in Rinker's (2015) study. Only $30 \%$ of the EMTs in this study fell into a comparable range.

The EMTs in this study showed the greatest struggle in being able to utilize their own emotions, while they found it much easier to manage their own and others' emotions and in the ability to perceive emotions. While it may be difficult to understand the difference in the EMTs inability to utilize their own emotions and their ability to manage their emotions, the difference falls in the fact that while EMTs are called to be able to manage emotions on the scene of a call in order to maintain control of the scene, they do not necessarily understand or know how to use emotions. It could also be an indicator that being able to manage both theirs and others' emotions serves to be effective for them since they do not know how to use the emotions that are present in either their own or others' lives. It is perhaps this, in conjunction with the need to control the scene, that provides the EMTs with the ability to avoid what they are witnessing and experiencing, and so they become great at this aspect of their job. This ability aligns with the avoidance that is seen when evaluating for PTSD in EMTs. It is quite likely that the 
inability to utilize emotions factors into the avoidance that EMTs demonstrate with regard to their traumatic calls.

With this study finding no differences between EMT-Bs and EMT-Ps on levels of emotional intelligence, it is difficult to identify the reason(s) for these differences. The difference in the emotional intelligence scores could be due to any number of factors. It is possible that EMTs with a greater number of years of the job and greater experience in the field demonstrate greater emotional intelligence, while those with fewer years and less experience have not quite achieved the exposure that could bring about increased emotional intelligence. It is also possible that older EMTs have greater levels of emotional intelligence as a result of lived experience, while those who are younger have lower levels of emotional intelligence. It is possible that levels of emotional intelligence are due to the ability of the EMTs to be open to and knowledgeable about the experiences of others, or that greater emotional intelligence is demonstrated in those who have parents, spouses, or families who have exposed them to greater emotional awareness; that they live in a culture in which they have been trained to be more aware of those around them and their experiences, as well as their impact on others. It could be that those with greater emotional intelligence have received counseling or training on emotional intelligence.

\section{Resilience}

The EMTs fell into both the highest $25 \%$ of the population and into the lowest $25 \%$ of the population (38\%) on a measure of resiliency (38\%), and EMT-B's were shown to be nearly one-and-three-fourths times less resilient than EMT-P's. The fact that EMT-B's fell into the lowest $25 \%$ of the population would appear to indicate that those 
with greater time on the job demonstrate greater levels of resiliency; however, it is quite possible that the scope of practice is a determinant of resiliency, rather than the amount of time in the field due to EMT-P's having a greater scope of practice and being exposed to more severe injury, which may either result in EMT-P's building up a greater ability to respond to difficult situations or the ability to "shut off," as one participant described his experience, in difficult situations. It is also possible that EMT-P's are more accustomed to the disenfranchisement experienced in the private sector than EMT-B's by virtue of the fact that they have worked more time in the field and have chosen to continue providing services despite the zeitgeist in the field regarding private versus public sector EMTs.

Fjeldheim, Nöthling, Pretorius, Basson, Ganasen. Heneke Cloete, and Seedat (2014) found that lower rates of resilience significantly predicted PTSD. Additionally, Stone (1998) found that the hardiness component of resiliency predicted PTSD in Paramedics when social support was not present. While these studies demonstrate the significance of resiliency in PTSD symptom expression, they do not address resiliency levels in EMTs or breakdown EMT resiliency levels among EMT levels. Therefore, this study provides an additional breadth to the PTSD literature, and allows for an additional area of further exploration for future researchers.

\section{Attachment}

The greatest percentage of the EMTs in this study were found to be securely attached a little bit, and EMT-B's were found to have both significantly more ambivalent attachment and significantly more anxious attachment. While the primary attachment style for the EMTs was secure attachment, it is notable that they were only securely attached "a little bit." Because the components of secure attachment are feeling a sense 
of comfort about life in general when they are close to someone, feeling relaxed and good when they are close to someone, and feeling a source of strength for other activities when they are close to someone, it is clear that there is a desire to be close to others; however, the nature of their job, and the resultant outcome of avoidance, as seen in their PTSD symptoms, would make this a difficult task. It is likely that it takes years before EMTs are able to manage their ability to attach to others while maintaining the ability to do their job with the risks they face, the disenfranchisement they experience, and the ability to continue on regardless of their circumstances. For this reason, EMT-B's demonstrate significantly more ambivalent attachment and significantly more anxious attachment due to their limited time on the job, and the limited opportunity to develop those skills.

Escolas, Escolas, and Bartone (2014) identified secure attachment to be associated with hardiness with an active duty military population. Additionally, Negri (2018) cited a study by Malach-Pines (2004), in which anxious and/or avoidant attachment was found to be associated with both burnout and poor coping. Benoit, Bouthillier, Moss, Rousseau, and Brunet (2010) propose that it is the individual's attachment, whether that be insecure or secure, that comes into play when an individual is exposed to a threatening or stressful situation. While these results are informative, they do not address attachment in EMTs. Further, an examination of the literature resulted in no studies examining attachment styles in EMS personnel. Therefore, as with several of the previous variables, the results of this research question provide information not available prior to this research study, and allow for an additional area of further exploration by future researchers. 


\section{Research Question Five}

Linear regression analysis results indicated that the most significant factors predicting PTSD were the use of avoidance coping and having ambivalent and anxious attachment styles, and that EMTs with higher PTSD scores were more likely to be those with higher scores in Avoidance Coping and Ambivalence Merger (Anxious) Attachment, but lower scores in Ambivalence-Worry (Ambivalent) Attachment scores. Of the three, the most important predictor was Avoidance Coping. Because the greatest percentage of EMTs utilized Positive Thinking as their Coping strategy, the primary percentage of EMTs did not fall into a high risk category for PTSD; however, there was a small portion of EMTs who did utilize Avoidance Coping as their coping strategy, placing them as high risk for developing PTSD. The results also indicated that EMT-B's were at a greater risk for developing PTSD due to them having both significantly more ambivalent attachment and significantly more anxious attachment. As with the previous discussion related to these results, it is likely that EMT-B's have not have enough time on the job to develop the ability to either identify better coping skills, or to be able to "shut off" the effects of their jobs as the more seasoned veterans demonstrated the ability to do.

In a study on military veterans, Zakin, Solomon, and Neria (2003) found a connection between attachment styles and PTSD symptoms. Additionally, attachment style has been suggested to be related to the development of trauma-related symptoms, (Alexander, 1992; Muller, Sicoli, \& Lemieux, 2000). Individuals with attachment avoidance and attachment anxiety demonstrated greater levels of PTSD in a study by Busuito, Huth-Bocks, and Puro (2014) as well. A meta-analysis performed by Woodhouse, Ayers, and Field (2015) once again confirmed the association between 
insecure attachment and PTSD symptoms. Multiple other studies with various populations demonstrate the same connection between anxious and insecure attachment and PTSD symptomatology, including civilians in Southern Israel exposed to terrorist attacks (Besser, Neria, \& Haynes, 2009), women with gynecological cancer (Holt, Jensen, Hansen, Elklit, \& Mogensen, 2016), individuals with chronic whiplash-associated disorder (Andersen, Elklit, \& Vase, 2011), Vietnam combat veterans (Nye, Katzman, Bell, Kilpatrick, Brainard, \& Haaland, 2008), university staff and students (Arikan, Stopa, Carnelley, \& Karl, 2016), civilians exposed to the Israel-Gaza war in 2009 (Avi, \& Yuval, 2010), security guards (Bogaerts, Daalder, Van Der Knaap, Kunst, \& Buschman, 2009), female healthcare workers in a longitudinal study on violence and health (La Flair, Bradshaw, Mendelson, \& Campbell, 2015), and school children following a schoolshooting trauma in Finland (Turunen, Haravouri, Punamaki, Suomalainen, \& Marttunen, 2014).

Mikulincer, Solomon, Shaver, and Ein-Dor (2014) propose that having secure attachment leads to resilience when one encounters stressful situations. Individuals with attachment avoidance and attachment anxiety demonstrated greater levels of PTSD in a study by Busuito, Huth-Bocks, and Puro (2014). Clark and Owens (2012) found the same relationship between anxious attachment in romantic relationships and PTSD symptom severity in U.S. veterans of Iraq and Afghanistan. They report that secure relationship attachment style is a protective factor for this population (Clark \& Owens, 2012). A meta-analysis performed by Woodhouse, Ayers, and Field (2015) once again confirmed the association between insecure attachment and PTSD symptoms. 
With regard to coping, a 2012 study examined refugees that had settled in the Netherlands following war, persecution, and violence, with the study outcomes again demonstrating a link between PTSD severity and avoidant coping (Huijts, Kleijn, can Emmerik, Noordhof, \& Smith, 2012). These same results were found in a study on Israeli veterans who had been diagnosed with PTSD (Galor, \& Hentschel, 2012). The final study published in 2012 was performed on veterans who had been exposed to traumatic situations, and who were receiving mental health care services through the Veterans Administration (VA). While this study identified emotional avoidance to be associated with PTSD symptom severity, the researchers also found that low levels of emotional expression was also associated with PTSD symptom severity (Hassija, Luterek, Naragon-Gainey, Moore, \& Simpson, 2012). Avoidant coping was the only resulting factor that demonstrated a significant association with changes in PTSD symptom severity over a 1-year period among veterans who had served in combat zones (Lee, Possemato, \& Ouimette, 2017). The use of spiritual coping has also shown to be protective as a factor against developing PTSD symptoms (Bryant-Davis \& Wong, 2013). Studies of emergency responders and armed forces personnel exposed to traumatic events have found an association between negative coping styles and psychological distress (LeBlanc, Regehr, Jelley \& Barath, 2008). The role of avoidance has been implicated in not only mediating between exposure and development, but it has also been implicated as a factor in maintaining PTSD symptomology (Maack, Tull, \& Gratz 2012).

The present study in private sector EMTs confirms each of the above listed results related to both attachment and coping. However, with the unique contribution of this study being the fact that the four variables: coping style; emotional intelligence; 
attachment; and resilience, have never been examined in conjunction with each other on PTSD, it is also important to discuss how resiliency and emotional intelligence impact the EMTs in this study in addition to discussing coping and attachment.

Wagner and Martin (2012) examined emotional intelligence and proactive coping on mental health in firefighters, with their results indicating a negative relationship between emotional intelligence and traumatic stress symptomatology. When examining the results of this study, it is notable that $58 \%$ of the EMTs fell into the average range on emotional intelligence, with only $30 \%$ falling into the unusually high range. This means that the greatest percentage of EMTs would benefit from training on emotional intelligence in order to increase their emotional intelligence levels since there is a direct protective connection between emotional intelligence and PTSD.

With regard to resiliency, lower resiliency was found to be significantly predictive of PTSD in a study by Fjeldheim, Nöthling, Pretorius, Basson, Ganasen. Heneke Cloete, and Seedat (2014). Additionally, the lack of social support, such as private sector EMTs experience, is predictive of PTSD according to a study by Stone (1998). However, because work-related social support assists in promoting resiliency following critical incidents (Prati \& Pietrantoni, 2010), and resilience has been found to be protective against PTSD symptomatology (Prati \& Pietrantoni, 2010), it may be important to encourage EMT-P's to provide that work-related support since their resiliency levels were found to be within the highest $25 \%$ of the population.

The greater levels of resiliency may possibly explain why only $30 \%$ of the EMTP's met DSM-5 criteria for PTSD, while 40\% of EMT-B's met criteria. Without knowing whether EMT-B's or EMT-P's were the higher scorers on emotional 
intelligence, it is unclear whether these levels factored into the differences seen between EMT-B's and EMT-P's. However, it is clear that neither EMT-B's nor EMT-P's use avoidance coping as their primary method of coping; therefore, this appears to be an additional protective factor shown in these study results.

While avoidance coping and both anxious and ambivalent attachment were the best predictors of PTSD, the EMTs in this study use coping and are securely attached only “a little bit." Even though EMT-B's use significantly more anxious and ambivalent attachment, the rates at which these are utilized could account for the fact that the levels of PTSD are not higher in this population.

Overall, because this study remains the only study to examine the four variables: coping style; emotional intelligence; attachment; and resilience in private sector EMTs, these results greatly enhance the knowledge in the field with regard to both PTSD and with regard to PTSD in private sector EMTs.

\section{Conclusions}

The experience of private sector EMTs is clearly one of disenfranchisement, which is likely to impact their rates of PTSD. Additional research needs to be undertaken and public education initiated in relation to this population, as well as therapeutic focus on the disenfranchisement component in conjunction with the PTSD treatment.

\section{Limitations of the Study}

The limitations for this study include the limited generalizability of the study results. Because the sample size was small, and because it consisted solely of private sector EMTs, the results may only be interpreted and generalized to this sector of the field. This limitation included the inability to collect from 300 EMTs, and the resultant 
outcome of the ability to collect only 50 valid responses. Another limitation could include problems related to the collection of data via online collection methods and the use of self-report measures. These could include the lack of participation by those individuals for whom it is not possible to participate via online surveys, but who may have been available for participation through the use of hard copy use of survey materials, as well answers that could be exaggerated or respondent biases. Further, because this study utilized non-experimental methods, there remains the inability to make use of cause-and-effect data that would have been possible had an experimental design been employed.

\section{Implications and Recommendations}

\section{Implications for the Field}

The results of the quantitative portion of the study noted that approximately $30 \%$ of the EMTs are at a high risk for PTSD. Common treatments for PTSD include Cognitive Behavioral Therapy (CBT) to assist in dealing with symptoms of hyperarousal, negative cognitions, and avoidant behaviors (Antunes-Alves \& Comeau, 2014), as well as in using conditional response treatments similar to the process used by Pavlov, in which a conditioned stimulus (CS), such as the fear response, being paired with an unconditioned stimulus (US), such as mild shocks, thereby resulting in the successful association of the US with the CS, and extinguishing the undesirable fear response (Singewald, Schmuckermair, Whittle, Holmes, \& Resler, 2014). Pharmacological agents have also been used in an attempt to extinguish the learned fear response through the use of benzodiazepines (anxiolytics), as are used primarily as "complementary techniques; electro-physiology, immediate early gene mapping, tracing studies, lesioning/inactivation 
approaches, and optogenetics)" (Singewald, Schmuckermair, Whittle, Holmes, \& Resler, 2014, p. 4). Finally, family therapy has been shown to be useful in assisting families in dealing with PTSD (Dekel \& Monson, 2010). Biofeedback is another option as a treatment for PTSD. Zucker, Samuelson, Muench, Greenberg, and Gevirtz (2009) found reduced PTSD symptoms with biofeedback treatment.

In addition to therapeutic treatment, it is important to address crisis issues that may arise immediately following exposure to a traumatic event. In this case, crisis intervention is relevant, and can include such services as Critical Incident Stress Debriefing (CISD), Critical Incident Stress Management (CISM), specified training in Disaster Behavioral Health (DBH), and therapeutic crisis intervention. According to Barboza (2005), CISD is the actual process of debriefing following the exposure to the traumatic incident, whereas CISM is an intervention system with multiple components, and is meant to be secondary to the initial intervention and support provided by crisis intervention strategies, with both meant to address the cognitive and emotional response to the traumatic stressor. Bledsoe (2003) indicates that CISD was intended to be a means by which those impacted by traumatic stressors would be allowed an emotional release through the retelling of the traumatic event. On the other hand, the goal of CISM is to reduce the severity, duration, and impairment from the traumatic event (Bledsoe, 2003). However, there has been some debate regarding not only the effectiveness of CISD and CISM, but whether these interventions are actually harmful (Regel, 2007; Bledsoe, 2003; Everly \& Mitchell, 2000). While critics have asserted that CISD and CISM is an ineffective treatment, multiple researchers have demonstrated that these are effective 
interventions (Regel, 2007; Meyers \& Wee, 2005; Bledsoe, 2003; Everly \& Mitchell, 2000).

Because EMTs are among the population of first responders who are at a risk for PTSD, it is important for them to reduce their risk for PTSD by strengthening the factors that are protective against PTSD. With one-third of the EMTs being at a high risk for developing PTSD, and with the profile of the EMTs in this study being that most have average emotional intelligence, use attachment "a little," and with it being "often true" that they are resilient, it is important to address each of these in both therapeutic and nontherapeutic ways to increase protective factors in each of these areas. Higher levels of emotional intelligence is one of those protective factors; therefore, even though the greatest percentage of EMTs fell into the average range on emotional intelligence, it is recommended that increasing their levels of emotional intelligence through training and practice in this area be sought out either on a personal level, or as the result of employers implementing this training as a part of their on the job training. Resiliency is another protective factor, with those having higher levels of resiliency being at lower risk for PTSD. As with emotional intelligence, resiliency training should be sought out, including stress management, self-management, and self-care as a few components of that training. However, the most important risk factors in predicting PTSD include avoidance coping, and having both ambivalent and anxious attachment styles. Counseling to address coping is recommended to enable the EMT in identifying and effectively utilizing adequate coping skills. Finally, to address attachment needs, individual and/or family counseling is recommended to identify the base for the current 
attachment style for those with ambivalent and anxious attachment styles, and enable the EMT to process through the issues present and to develop more secure attachment styles. In addition to the specific treatments that have been shown to be effective in working with those exposed to traumatic events, there is a need for advocacy in the community and with insurance companies for these individuals to obtain more than just a few "crisis management" sessions to address their needs. Rather, there needs to be an emphasis on additional sessions so that the treatment for this population to be complete. Additionally, preventative measures are imperative for this population, so that when exposure takes place, there are already supportive systems in place to mitigate the impact of that exposure. This prevention aspect would likely be effective as an on-the-job training component for all employees, field employees, and management and supervisory employees alike; field employees because they are the individuals exposed to the traumatic events, and management and supervisory employees in order to implement an awareness component so that they are able to adequately and effectively manage the procedures addressing the needs of employees who are exposed to traumatic events. It is also recommended that specified training related to traumatic events be instituted as a part of both EMT-Basic and EMT-Paramedic training in order to 1) make EMTs aware of what they are likely to be exposed to, and what is likely to be a part of their job, and 2) to provide them with foundational training to assist them in being prepared to this type of exposure in their jobs, just as the other aspects of EMT Basic and Paramedic training prepares them for being adequate providers of emergency medical services.

Because of the revelation that this population demonstrates disenfranchisement that is not present in EMTs working in the public sector of the field, the inclusion of the 
disenfranchisement component is an important consideration for clinical treatment. There has been a wealth of research on the public sector, which has resulted in treatment implications. Should EMTs working in the private sector present for treatment, it is likely that treatment providers could assume that treatment would take the same clinical course as with EMTs presenting for treatment who work in the public sector of the field. This, of course, as shown in the results of this study, would be an egregious error should the disenfranchisement not be included as a component of the treatment course for private sector EMTs.

Additionally, multiple other differences were revealed, including the lack of support for EMTs in the private sector, the lack of financial stability within the employment sector, and the lack of retirement options. Each of these would have a tremendous impact on the treatment course with regard to the nuances present for private sector EMTs. This leaves a great need open for clinicians to become well versed in the work structure and environment for private sector EMTs so that clinical treatment may be tailored to those specific needs. Additionally, one goal of clinical treatment should be aimed at evaluating the level of disenfranchisement the EMT is experiencing if this is present, since not all EMTs may experience disenfranchisement depending on whether they are new to the field, whether they have previously worked in the public sector, and whether lack of societal acknowledgement is important to them or not.

Because the EMTs also indicated a lack of support by their own management system in terms of the lack of ability to take breaks, the running of calls on a back-toback basis, and the lack of viable resources to deal with the exposure to traumatic events, advocacy in the private sector of the field is urgent. It is possible that this component has 
had tremendous implications regarding those who develop PTSD and those who do not. This advocacy could take place in terms of an educational component for management and supervisory staff regarding the risk to their employees' physical, emotional, and mental health, as well as an evaluation related to employee sick days and time off from work as a result of the lack of supportive components that impacts their financial bottom line. Because private the private sector is privately funded, and due to the fact that they are for profit agencies that benefit from employees continuing to run calls despite any trauma that they have been exposed to, as well as due to the fact that running with as few units as possible to increase their bottom line, that impact to the financial bottom line could be a significant motivator to management in addressing the needs of their employees related to the exposure and response to traumatic events.

Finally, it is important to note that because of the EMS culture, EMTs are not likely to present for treatment with needs related to PTSD. Rather, it is more likely that EMTs will present for treatment due to martial problems and/or marital failure, substance abuse or addiction, or problems related to their job. Part of the EMS culture as presented previously indicated that dealing with their employee's exposure to trauma was not a high priority for management within the private sector; however, when the work of EMTs is affected by their marital problems, by substance use or addiction, or when they are unable to work due to mental health issues such as anxiety or depression, they are referred for very short-term counseling through their Employee Assistance Program (EAP). Therefore, therapists need to be aware that they need to include work-related issues as a part of their screening process related to symptoms and diagnosis. This includes the knowledge that the tools for assessing individuals coming in for treatment 
from the public sector are not sufficient enough to meet the diagnostic and treatment needs of those who work, or who have in the past worked in the private sector. Addressing both the disenfranchisement component, as well as the additional component of how management-related stressors and on-the-job requirements pushing EMTs to continue working despite exposure to traumatic events as a result of the private sector being a for-profit entity, and how this may impact the advancement of and the possible development of PTSD in private sector EMTs is greatly important.

\section{Implications and Recommendations for Future Research}

Because of the lack of research on private sector EMTs, there remains the need for an evaluation of this population in terms of unique characteristics and intricacies that would be appropriate for mental health treatment. The implications for future research include the need for further research to be performed on this population of providers. With the small sample size for this study, it would do well to replicate the current study with a larger sample size to verify the research results found here, as well as to develop a "theory" of coping of PTSD among private sector EMTs. It would also be beneficial to perform comparison studies evaluating private and public sector EMTs within the same research study, utilizing the same survey instruments to evaluate for differences among the two populations, and to evaluate for significance of any differences found within the study. It would also be beneficial if future studies examined EMTs practicing in rural areas versus those practicing in metropolitan areas or larger, major cities such as Los Angeles or New York.

Because the EMTs in this study noted a lack of support by their administrative staff, and due to the fact that these EMTs indicated the inability to take breaks, have 
down time, or destress even following traumatic incidents, there is also a need to evaluate for this component in private sector EMTs to identify whether these have an impact on PTSD for this population of EMTs. Due to the need to limit the number of variables in in order for this study to provide a baseline study for further researchers to build upon, a number of areas were left open to examination. These include an examination of EMTs with regard to SES, ethnicity, acculturation, gender, and the impact on females of working in a male dominated field. While the current study provided for the addition to the knowledge in the field with regard to private sector EMTs, there remains a great deal of information yet to glean.

Additional recommendations would be to run statistical analyses for emotional intelligence evaluating age, number of years in the field, number of years in relationship, and previous relational or interpersonal counseling against emotional intelligence to identify any significant correlations, and then to evaluate these by the same statistical means used in this study related to PTSD. It would also be recommended to run statistical analyses for resiliency evaluating time on the job, scope of practice against resiliency to identify any significant correlations, and then to evaluate these by the same statistical means used in this study related to PTSD, as well as to examine EMT-B's against their previous experience in other sectors of the field to evaluate for resiliency to identify any significant correlations, and then to evaluate these by the same statistical means used in this study related to PTSD. With regard to the study results with Avoidance Coping indicating the greatest risk for PTSD, it would be good to evaluate whether EMT-B's had the greatest levels of avoidance coping since they had significantly greater levels of anxious attachment and avoidant attachment. Finally, it is recommended 
that post-traumatic growth and compassion satisfaction be included as an additional set of variables for consideration for further studies in this population of first responders. 


\section{References}

Acheson, D.T., Gresack, J.E., \& Risbrough, V.B. (2012). Hippocampal dysfunction effects on context memory: Possible etiology for post-traumatic stress disorder. Neuropharmacology, 62(2), 674-685.

Agaibi, C. \& Wilson, J.P. (2005). Trauma, PTSD, and resilience. Trauma, Violence, and Abuse, 6(3), 195-216.

Agaibi, C. (2003). Understanding resilience to the effects of traumatic stress. Master's thesis, Cleveland State University, Cleveland, $\mathrm{OH}$.

Ainsworth, M. D. S., \& Bell, S. M. (1970). Attachment, exploration, and separation: Illustrated by the behavior of one-year-olds in a strange situation. Child Development, 41, 49-67.

Ainsworth, M. D., Blehar, M. C., Waters, E., \& Wall, S. (1978). Patterns of attachment: A psychological study of the strange situation. Hillsdale, N.J., Erlbaum.

Aldwin, C., \& Revenson, T.A. (1987). Does coping help? A reexamination of the relation between coping and mental health. Journal of Personality and Social Psychology, 53, 337-348.

Aldwin, C., Folkman, S., Schaefer, C., Coyne, J.C., \& Lazarus, R.S. (1980, September). Ways of coping: A process measure. Presented at the 88th annual meeting of the American Psychological Association, Montreal, Quebec, Canada.

Alexander, P.C. (1992). Application of attachment theory to the study of sexual abuse. Journal of Consulting and Clinical Psychology, 60, 185-195. 
Ali, D.M. (2016). Strategies for preventing suicides in the fire service. Retrieved 12/9/2017 from http://www.fireengineering.com/articles/print/volume-169/issue12/features/strategies-for-preventing-suicides-in-the-fire-service.html.

American Psychiatric Association (2000). Diagnostic and Statistical Manual of Mental Disorders, (4th ed., text rev.). Washington DC: Author.

American Psychiatric Association (2013). Diagnostic and Statistical Manual of Mental Disorders (5th ed.). Washington DC: Author.

Andersen, T.E., Elklit, A., \& Vase, L. (2011). The relationship between chronic whiplash-associated disorder and post-traumatic stress: Attach-anxiety may be a vulnerability factor. European Journal of Psychotraumatology, 2(5633), 1-9.

Antunes-Alves, S., \& Comeau, T. (2014). A clinician's guide to the neurobiology underlying the presentation and treatment of PTSD and subsequent growth. Archives of Psychiatry and Psychotherapy, 3, 9-17.

Arikan, G., Stopa, L., Carnelley, K.B., \& Karl, A. (2016). The associations between adult attachment, posttraumatic symptoms, and posttraumatic growth. Anxiety, Stress, \& Coping, 29(1), 1-20.

Avi, B., \& Yuval, N. (2010). The effects of insecure attachment orientations and perceived social support on posttraumatic stress and depressive symptoms among civilians exposed to the 2009 Israel-Gaza war: A follow-up Cross-Lagged panel design study. Journal of Research in Personality, 44(3), 335-341.

Avraham, N., Goldblatt, H., \& Yafe, E. (2014). Paramedics' Experiences and Coping Strategies When Encountering Critical Incidents. Qualitative Health Research, 24(2), 194-208. 
Ayers, T.S., Sandler, I.N., West, S.G., \& Roosa, M.W. (1996). A dispositional and situational assessment of children's coping: Testing alternative models of coping. Journal of Personality, 64(4), 923-958.

Babbie, E.R. (2010). The Practice of Social Research (12th ed.). Belmont, CA: Wadsworth Cengage.

Bacharach, S. B., Bamberger, P. A., \& Doveh, E. (2008). Firefighters, critical incidents, and drinking to cope: The adequacy of unit-level performance resources as a source of vulnerability and protection. Journal of Applied Psychology, 93(1), 155-169.

Baek, H-S., Lee, K-U., Joo, E-J., \& Lee, M-Y., Choi, K-S. (2010). Reliability and validity of the Korean version of the Connor-Davidson Resilience Scale (K-CDRISC). Psychiatric Investigation: Official Journal of the Korean Neuropsychiatric Association, 7, 109-115.

Bar-On, R. (1997). Bar-On Emotional Quotient Inventory (EQ-i): Technical manual. Toronto: Multi-Health Systems.

Bar-On, R. (2000). Emotional and social intelligence: Insights from the Emotional Quotient Inventory. In R. Bar-On \& J.D.A. Parker (Eds.), The handbook of emotional intelligence: Theory, development, assessment, and the application at home, school and in the workplace (pp. 363-388). San Francisco: Jossey-Bass Inc.

Bar-On, R. (2006). The Bar-On model of emotional-social intelligence (ESI). Psicothema, 18, 13-25. 
Beck, J.S. (2011). Cognitive therapy: Basics and beyond (2nd ed.) New York, NY: The Guilford Press.

Beckman, S.A. (2015). Secondary Traumatic Stress and Posttraumatic Growth: Risk and Protective Factors among American Red Cross Disaster Responders and Disaster Mental Health Workers. (Publication No. 3728149) [Doctoral Dissertation, University of Minnesota]. ProQuest Dissertations and Theses Global.

Bennett, P., Williams, Y., Page, N., Hood, K., Woollard, M., \& Vetter, N. (2005). Associations between organizational and incident factors and emotional distress in emergency ambulance personnel. British Journal of Clinical Psychology, 44, 215-226.

Benoit, M., Bouthillier, D., Moss, E., Rousseau, C., \& Brunet, A. (2010). Emotion regulation strategies as mediators of the association between level of attachment security and PTSD symptoms following trauma in adulthood. Anxiety, Stress, \& Coping, 23(1), 101-118.

Besser, A., Neria, Y., \& Haynes, M. (2009). Adult attachment, perceived stress, and PTSD among civilians exposed to ongoing terrorist attacks in Southern Israel. Personality \& Individual Differences, 47(8), 851-857.

Billings, A.G., \& Moos, R.H. (1981). The role of coping responses in attenuating the impact of stressful life events. Journal of Behavioral Medicine, 4, 139-157.

Blevins, C.A., Weathers, F.W., Davis, M.T., Witte, T.K., \& Domino, J.L. (2015). The Posttraumatic Stress Disorder Checklist for DSM-5 (PCL-5): Development and initial psychometric evaluation. Journal of Traumatic Stress, 28(6), 489-498. doi: 10.1002/jts.22059. Epub 2015 Nov 25. 
Bodzy, M.E., Barreto, S.J., Swenson, L.P., Liguori, G., \& Costea, G. (2016). Selfreported psychopathology, trauma symptoms, and emotion coping among child suicide attempters and ideators: An exploratory study of young children. Archives of Suicide Research, 20, 160-175.

Bogaerts, S., Daalder, A.L., Van Der Knaap, L.M., Kunst, M.J.J.M., \& Buschman, J. (2009). Critical incident, adult attachment style, and posttraumatic stress disorder: A comparison of three groups of security workers. Social Behavior and Personality, 36(8), 1063-1072.

Bomyea, J., Risbrough, V., \& Lang, A.J. (2012). A consideration of select pre-trauma factors as key vulnerabilities in PTSD. Clinical Psychology Review, 32(7), 630641.

Boss, P. (1999). Ambiguous loss: Learning to live with unresolved grief. Cambridge, MA: Harvard University Press.

Bowlby, J. (1973). Attachment and loss, Volume 2: Separation: Anxiety and anger. New York, NY: Basic Books.

Bowlby, J. (1982). Attachment and loss: Retrospect and prospect. American Journal of Orthopsychiatry, 52(4), 664-678.

Brackbill, R.M., Hadler, J.L., DiGrande, L., Ekenga, C.C., Farfel, M.R., Friedman, S., Perlman, S.E., Stellman, S.D., Walker, D.J., Wu, D., Yu, S., \& Thorpe, L.E. (2009). Asthma and posttraumatic symptoms 5 to 6 years following exposure to the World Trade Center terrorist attack. Journal of the American Medical Association, 302(5), 502-528. 
Brackett, M. A., \& Geher, G. (2006). Measuring emotional intelligence: Paradigmatic diversity and common ground. In J. Ciarrochi, J. P. Forgas, \& J. D. Mayer (Eds.), Emotional intelligence in everyday life (2nd ed.), 27-50. New York, NY: Psychology Press.

Bramsen, I., van der Ploeg, H.M., \& Twisk, J.W.R. (2002). Secondary traumatization in Dutch couples of World War II survivors. Journal of Consulting and Clinical Psychology, 70(1), 241-245.

Breedlove, S.M., Rosenzweig, M.R., \& Watson, N.V. (2007). Biological psychology: An introduction to behavioral, cognitive, and clinical neuroscience (5th ed.). Sunderland, MA: Sinauer Associates.

Brennan, K.A., Clark, C.L., \& Shaver, P.R. (1998). Self-report measurement of adult attachment: An integrative overview. In J.A. Simpson \& W.S. Rholes (Eds.) Attachment theory and close relationships (pp. 46-76). New York: Guilford Press.

Brenner, L.A. (2011). Neuropsychological and neuroimaging findings in traumatic brain injury and post-traumatic stress disorder. Dialogues in Clinical Neuroscience, 13(3), 311-337.

Brewin, C.R., Dalgleish, T., \& Joseph, S. (1996). A dual representation theory of posttraumatic stress disorder. Psychological Review, 103(4), 670-686.

Brohawn, K.H., Offringa, R., Pfaff, D.L., Hughes, K.C., \& Shin, L.M. (2010). The neural correlates of emotional memory in posttraumatic stress disorder. Biological Psychiatry, 68(11), 1023-1030. 
Bryant, R.A., Marosszeky, J.E., Crooks, J., Baguley, I., \& Gurka, J. (2000). Coping style and post-traumatic stress disorder following severe traumatic brain injury. Brain Injury, 14(2), 175-180.

Bryant-Davis, T., \& Wong, E.C. (2013). Faith to move mountains: Religious coping, spirituality, and interpersonal trauma recovery. American Psychologist, 68(8), 675-685.

Burr, V. (2001). An introduction to Social Construction. London: Routledge.

Burr, V. (2004). Social constructionism (2nd ed.). London: Routledge.

Busuito, A., Huth-Bocks, A., and Puro, E. (2014). Romantic attachment as a moderator of the association between childhood abuse and posttraumatic stress disorder symptoms. Journal of Family Violence, 29, 567-577.

Calams, S. (October 23, 2017). Private vs. public ambulance services: What's the difference? https://www.ems1.com/private-public-dispute/articles/private-vspublic-ambulance-services-whats-the-difference-WTgJNJgR4K1lj1V9/\#: :text $=$ Therefore $\% 2 \mathrm{C} \% 20$ patients $\% 20$ are $\% 20$ only $\% 20$ billed,scheduled $\% 2 \mathrm{C} \% 20$ non $\%$ 2Demergency\%20transports.\&text=Private $\% 20$ ambulance $\% 20$ services $\% 20$ tend $\% 20$ to $\% 20$ have $\% 20$ the $\% 20$ lowest\%20rates\%20of\%20pay. Retrieved 8/17/2020.

Campbell-Sills, .L, Forde, D., Stein, M.B. (2009). Demographic and childhood environmental predictors of resilience in a community sample. Journal of Psychiatric Res, 43, 1007-1012.

Carrion, V.G., Weems, C.F., Richert, K., Hoffman, B.C., \& Reiss, A.L. (2010). Decreased prefrontal cortical volume associated with increased bedtime cortisol in traumatized youth. Biological Psychiatry, 68(5), 491-493. 
Carver, C.S. (1997a). Adult attachment and personality: Converging evidence and a new measure. Personality \& Social Psychology Bulletin, 23, 865-883.

Carver, C. S. (1997b). You want to measure coping but your protocol's too long: Consider the Brief COPE. International Journal of Behavioral Medicine, 4, 92100.

Carver, C. S., \& Scheier, M. F. (1994). Situational coping and coping dispositions in a stressful transaction. Journal of Personality and Social Psychology, 66(1), 184195.

Carver, C.S., Scheier, M.F., \& Weintraub, J.K. (1989). Assessing coping strategies: A theoretically based approach. Journal of Personality and Social Psychology, $56(2), 267-283$.

Caska, C.M., Smith, T.W., Renshaw, K.D., Allen, S.N., Uchino, B.N., \& Carlisle, M. (2014). Posttraumatic stress disorder and responses to couple conflict: Implications of cardiovascular risk. Health Psychology, 33(11), 1273-1280.

Chang, K., \& Taormina, R.J. (2011). Reduced secondary trauma among Chinese earthquake rescuers: A test of correlates and life indicators. Journal of Loss and Trauma, 16, 542-562.

Chantrell, S. (2009). Growth in emotional intelligence. Psychotherapy with a learning disabled girl. Journal of Child Psychotherapy, 35(2), 157-174.

Chun Tie, Y., Birks, M., \& Francis, K. (2019). Grounded theory research: A design framework for novice researchers. Open Medicine, 7, 1-8. 
Ciarrochi, J., Deane, F.P., \& Anderson, S. (2002). Emotional intelligence moderates the relationship between stress and mental health. Personality and Individual Differences, 32, 197-209.

Clark, A.A., \& Owens, G.P. (2012). Attachment, personality characteristics, and posttraumatic stress disorder in U.S. veterans of Iraq and Afghanistan. Journal of Traumatic Stress, 25, 657-664.

Compas, B.E., Connor-Smith, J.K., Saltzman, H., Harding Thomsen, A., \& Wadsworth, M.E. (2001). Coping with stress during childhood and adolescence: Problems, progress, and potential in theory and research. Psychological Bulletin, 127, 87127.

Connor, K.M. \& Davidson, J.R.T. (2003). Development of a new resilience scale: The Connor-Davidson Resilience Scale (CD-RISC). Depression and Anxiety, 18, 7682.

Connor-Smith, J.K., Compas, B.E., Wadsworth, M.E., Harding Thomsen, A., \& Saltzman, H. (2000). Responses to stress in adolescence: Measurement of coping and involuntary stress response. Journal of Consulting and Clinical Psychology, $68,976-992$.

Consortium for Research on Emotional Intelligence in Organizations (2017). http://www.eiconsortium.org/measures/sreis.html. Retrieved April 2, 2018.

Coyne, J.C., Aldwin, C., \& Lazarus, R.S. (1981). Depression and coping in stressful episodes. Journal of Abnormal Psychology, 90, 439-447.

Creswell, J.W. (1998). Qualitative inquiry and research design: Choosing among five traditions. Thousand Oaks, CA: Sage. 
Crosbie-Burnett, M., Lewis, E.A., Sullivan, S., Podolsky, J., Mantilla de Souza, R., \& Mitrani, V. (2005). Advancing theory through research. In V. L. Bengston, A.C. Acock, K.R. Allen, P. Dilworth-Anderson, \& D.M. Klein (Eds.), Sourcebook of family theory \& Research. Thousand Oaks, CA: Sage.

Cyprus, B.S. (2017). Rigor or reliability and validity in qualitative research:

Perspectives, strategies, reconceptualization, and recommendations. Dimensions of Critical Care Nursing, 36(4), 253-263.

Dahl, C.M., \& Boss, P. (2005). The use of phenomenology for family therapy research: The search for meaning. In D.H. Sprenkle and F.P. Piercy (Eds.), Research methods in family therapy (2nd ed., pp. 85-99). New York, NY: Guilford.

Dalgleish, T., Meiser-Stedman, R., \& Smith, P. (2005). Cognitive aspects of posttraumatic stress reactions and their treatment in children and adolescents: An empirical review and some recommendations. Behavioral and Cognitive Psychotherapy, 33, 459-486.

Dass-Brailsford, P. (2010). Secondary trauma among disaster responders: The need for self-care. In Dass-Brailsford, P. (Ed.), Crisis and Disaster Counseling: Lessons Learned from Hurricane Katrina and Other Disasters (pp. 213-228). Thousand Oaks: SAGE Publications, Inc.

Davidson, J.R.T., \& Connor, K.M. (2018). Connor-Davidson Resilience Scale (CDRISC) Manual. Unpublished. 01-01-2018 and partly accessible atwww.cdrisc.com. 
Declercq, F., \& Willemsen, J. (2006). Distress and post-traumatic stress disorders in high risk professionals: Adult attachment style and the dimensions of anxiety and avoidance. Clinical Psychology and Psychotherapy, 13, 256-263.

DeFranzo, S.E. (2012). Retrieved from https://www.snapsurveys.com/blog/4-mainbenefits-survey-research/ May 25, 2018.

Dekel, R., \& Monson, C.M. (2010). Military-related post-traumatic stress disorder and family relations: Current knowledge and future directions. Aggression and Violent Behavior, 15, 303-309.

Del Ben, K.S., Scotti, J.R., Chen, Y., \& Fortson, B.L. (2006). Prevalence of posttraumatic stress disorder symptoms in firefighters. Work and Stress, 20(1), $37-48$.

Dellinger, A.B., \& Leech, N.L. (2007). Toward a unified validation framework in mixed methods research. Journal of Mixed Methods Research, 1(4), 309-332.

Denzin, N., \& Lincoln, Y. (2003). The discipline and practice of qualitative research. In N.K. Denzin \& Y.S. Lincoln (Eds.), The landscape of qualitative research $\left(2^{\text {nd }}\right.$ ed.) (pp. 1-45). Thousand Oaks, CA: Sage.

Dineberg, R.E., Mccaslin, S.E., Bates, M.N., \& Cohen, B.E. (2014). Social support may protect against the development of posttraumatic stress disorder: Findings from the Heart and Soul Study. American Journal of Health Promotion, 28(5), 294297.

Donoghue, K. J. (2004). Measuring coping: Evaluating the psychometric properties of the COPE. Retrieved from http://ro.ecu.edu.au/theses_hons/968. 
Ebata, A.T., and Moos, R.H. (1991). Coping and adjustment in distressed and healthy adolescents. Journal of Applied Developmental Psychology, 12, 33-54.

Ein-Dor, T., Doron, G., Mikulincer, M., Solomon, Z., \& Shaver, P.R. (2010). Together in pain: Attachment-Related dyadic processes and posttraumatic stress disorder. Journal of Counseling Psychology, 57(3), 317-327.

Elwood, L.S., \& Williams, N.L. (2007). PTSD-related cognitions and romantic attachment style as moderators of psychological symptoms in victims of interpersonal trauma. Journal of Social and Clinical Psychology, 26(10), 11891209.

Escolas, S., Escolas, H., \& Bartone, P. T. (2014). Adult attachment style, hardiness, and mood. Military Behavioral Health, 2, 129-137.

Fernández, P. (2011). Anxiety of nursing staff in the face of death in critical care units and its relationship with the patients' age. Ansiedad del personal de enfermería, 22(3), 96-103.

Figley, C. R. (1995). Compassion fatigue as secondary traumatic stress disorder: An overview. In C. R. Figley (Ed.), Compassion fatigue. New York, NY: Brunner/Mazel.

Fjeldheimc C.B., Nöthling, J., Pretorius, K., Basson, M., Ganasen, K., Heneke, R., Cloete, K.J., \& Seedat, S. (2014). Trauma exposure, posttraumatic stress disorder, and the effect of explanatory variables in paramedic trainees. BMC Emergency Medicine, 14-11. 
Fleurkens, P., Rinck, M., \& Minnen A., (2014). Implicit and explicit avoidance in sexual trauma victims suffering from posttraumatic stress disorder: a pilot study. European Journal of Psychotruamatology, 5, Article ID 21359.

Flick, U. (2006). An introduction to qualitative research ( $3^{\text {rd }}$ ed.). Thousand Oaks, CA: Sage.

Folkman, S., \& Lazarus, R.S. (1980). An analysis of coping in a middle-aged community sample. Journal of Health and Social Behavior, 21, 219-239.

Folkman, S., \& Lazarus, R.S. (1985). If it changes it must be a process: A study of emotion and coping during three stages of a college examination. Journal of Personality and Social Psychology, 48, 150-170.

Folkman, S., Lazarus, R.S., Dunkel-Schetter, C., DeLongis, A., \& Green, R.J., (1986). Dynamics of a stressful encounter: Cognitive appraisal, coping, and encounter outcomes. Journal of Personality and Social Psychology, 50, 992-1003.

Forneris, C.A., Gartlehner, G., Brownley, K.A., Gaynes, B.N., Sonis, J., CokerSchwimmer, E., Jonas, D.E., Greenblatt, A., Wilkins, T.M., Woodell, C.L., \& Lohr, K.N. (2013). Interventions to prevent post-traumatic stress disorder: A systematic review. American Journal of Preventative Medicine, 44(6), 635-650.

Frederickson, B.L. (1998). What good are positive emotions? Review of General Psychology, 2, 300-319.

Frederickson, B.L. (2001). The role of positive emotions in positive psychology. The broaden and build theory of positive emotions. American Psychologist, 56(3), 218-226. 
Frederickson, B.L. (2002). Positive emotions. In C.R. Snyder \& S.J. Lopez (Eds.), Handbook of positive psychology (pp. 120-134). New York, NY: Oxford University Press.

Frye, B.A. (1995). Use of cultural themes in promoting health among Southeast Asian refugees. American Journal of Health Promotion, 9(4), 269-280.

Fullerton, C.S., Ursano, R.J., \& Wang, L. (2004). Acute stress disorder, posttraumatic stress disorder, and depression in disaster or rescue workers. American Journal of Psychiatry, 161(8):1370-1376.

Gaher, R.M., Hofman, N.L., Simons, J.S., \& Hunsaker, R. (2013). Emotion regulation deficits as mediators between trauma exposure and borderline symptoms. Cognitive Therapy Research, 37, 466-475.

Galor, S., \& Hentschel, U. (2012). Problem-solving tendencies, coping styles, and selfefficacy among Israeli veterans diagnosed with PTSD and depression. Journal of Loss and Trauma, 17, 522-535.

Galovski, T., \& Lyons, J.A. (2004). Psychological sequelae of combat violence: A review of the impact of PTSD on the veteran's family and possible interventions. Aggression and Violent Behavior, 9(5), 477-501.

Gergen, K.J. (1994). Realities and relationships: Soundings in social construction. Cambridge, MA: Harvard University Press.

Gergen, K.J. (2001). Social construction in context. London: Sage.

Germain, C.L., Kangas, M., Taylor, A., \& Forbes, D. (2016). The role of trauma-related cognitive processes in the relationship between combat-PTSD symptom severity and anger expression and control. Australian Journal of Psychology, 68, 73-81. 
Ghazali, S.R. (2014). Investigating the relationship between post-traumatic stress disorder (PTSD) symptoms and emotional intelligence among adolescent refugees from the Middle East. ASEAN Journal of Psychiatry, 15(2), 220-224.

Gil, S. (2005). Coping style in predicting posttraumatic stress disorder among Israeli students. Anxiety, Stress, and Coping, 18(4), 351-359.

Gilbertson, M.W., Paulus, L.A., Williston, S.K., Gurvits, T.V., Lasko, N.B., Shenton, M.E., et al. (2006). Neurocognitive function in monozygotic twins discordant for combat exposure: Relationship to posttraumatic stress disorder. Journal of Abnormal Psychology, 115, 484-495.

Grajales, T. (2013). Dissertation development handbook: How to write a dissertation proposal in education and psychology. Berrien Springs, MI: Andrews University.

Green, B.L., \& Kaltman, S.I. (2003). Recent research findings on the diagnosis of PTSD. In R. Simon (Ed.), Posttraumatic stress disorder in litigation: Guidelines for forensic assessment (2nd d.) (pp. 19-39). Washington: American Psychiatric Publishing, Inc.

Guba, E.G. \& Lincoln, Y.S. (1989). Fourth Generation Evaluation. Newbury Park, CA: Sage.

Guthrie, I. (2003). Qualitative Methods in Clinical Psychology (personal communication). Presented in Quarter lecture series presented at Loma Linda University, Loma Linda, CA.

Hagar, M.A., Wilson, S., Pollak, T.H., \& Rooney, P.M. (2003). Response Rates for Mail Surveys of Nonprofit Organizations: A Review and Empirical Test. Nonprofit and Voluntary Sector Quarterly, 32(2), 252-267. 
Halpern, J., Gurevich, M., Schwartz, B., \& Brazeau, P. (2009). What makes an incident critical for ambulance workers? Emotional outcomes and implications for intervention. Work and Stress, 23, 173-189.

Hart, R.H. (2003). A face of war: PTSD. Psychiatric Times, 20(6), 1-5.

Hassija, C.M., Luterek, J.A., Naragon-Gainey, K., Moore, S.A., \& Simpson, T. (2012). Impact of emotional approach coping and hope on PTSD and depression symptoms in a trauma exposed sample of veterans receiving outpatient VA mental health care services. Anxiety, Stress, and Coping, 25(5), 559-573.

Holt, K.A., Jensen, P.T., Hansen, D.G., Elklit, A., \& Mogensen, O. (2016). Rehabilitation of women with gynecological cancer: The association between adult attachment, post-traumatic stress disorder, and depression. PsychoOncology, 25, 691-698.

Hopper, J.W., Frewen, P.A., van der Kolk, B.A., Lanius, R.A. (2007). Neural correlates of re-experiencing, avoidance, and dissociation in PTSD: Symptom dimensions and emotion dysregulation in responses to script-driven trauma injury. Journal of Traumatic Stress, 20, 713-725.

Horn, S.R., Miller-Graff, L.E., Galano, M.M., \& Graham-Bermann, S.A. (2017). Posttraumatic stress disorder in children exposed to intimate partner violence: The clinical picture of physiological arousal symptoms. Child Care in Practice, 23(1), 90-103.

http://www.connordavidson-resiliencescale.com/about.php. Retrieved May 7, 2018. http://www.eiconsortium.org/measures/sreis.html. Retrieved May 3, 2018. 
Huijts, I., Kleijn, W.C., van Emmerik, A.A.P., Noordhof, A., \& Smith, A.J.M. (2012). Dealing with man-made trauma: The relationship between coping style, posttraumatic stress, and quality of life in resettled, traumatized refugees in the Netherlands. Journal of Traumatic Stress, 25, 71-78.

Janesick, V. (2000). The Choreography of Qualitative Research Design: Minuets, Improvisations, and Crystallization. In N.K. Denzin \& Y. S. Lincoln (Eds.), The Handbook of Qualitative Research (pp. 379-400). Thousand Oaks, California, Sage Publications.

Jamessen, B.H., Eid, J., Laberg, J.C., and Thayer, J.F. (2002). The effect of sensitization and coping style on post-traumatic stress symptoms and quality of life: Two longitudinal studies. Scandinavian Journal of Psychology, 43, 181-188.

Jonker, C.S., \& Vosloo, C. (2008). The psychometric properties of the Schutte Emotional Intelligence Scale. SA Journal of Industrial Psychology, 34(2), 21-30. Jung, Y.E., Min, J.A., Shin, A.Y., Han, S.Y., Lee, K.U., \& Kim, T.S., et al. (2012). The Korean version of the Connor-Davidson Resilience Scale: an extended validation. Stress Health, 28, 319-326.

Kaniasty, K., \& Norris, F.H. (1993). A test of the Social Support Deterioration Model in the context of natural disaster. Journal of Personality and Social Psychology, 64(3), 395-408.

Kanitz, E., Puppe, B., Tuchscherer, M., Heberer, M., Viergutz, T., \& Tuchscherer, A. (2009). A single exposure to social isolation in domestic piglets activates behavioural arousal, neuroendocrine stress hormones, and stress-related gene expression in the brain. Physiology \& Behavior, 98(1/2), 176-285. 
Kessler, R.C. (2000). Posttraumatic stress disorder: The burden to the individual and to society. The Journal of Clinical Psychiatry, 61(5), 4-12.

Kim, Y., \& Carver, C.S. (2007). Frequency and difficulty in caregiving among spouses of individuals with cancer: Effects of adult attachment and gender. PsychoOncology, 16, 714-723.

Knows-His-Gun, K., Bufford, R.K., Gathercoal, K., \& Seegobin, W. (2013). Resilience at St. Labre: Childhood adversity, spiritual well-being, employment, and resilience in a multi-ethnic rural population. Faculty publications - Graduate School of Clinical Psychology, Paper 4. 2013. http://digitalcommons.georgefox.edu/gscp_fac/4.

Kobayaski, K. (2001). Role of catecholamine signaling in brain and nervous system functions: New insights from mouse molecular genetic study. Journal of Investigative Dermatology Symposium Proceedings, 6(1), 115-121.

Kremen, W.S., Kornrn, K.C., Afari, N., \& Lyons, M.J. (2012). Twin studies of posttraumatic stress disorder: Differentiating vulnerability factors from squelae. Neuropharmacology, 62(2), 647-653.

Kubzansky, L.D., Koenen, K.C., Jones, C., \& Eaton, W.W. (2009). A prospective study of posttraumatic stress disorder symptoms and coronary heart disease in women. Health Psychology, 28(1), 125-130.

Kuiper, N.A. (2012). Humor and resiliency: Towards a process model of coping and growth. Europe's Journal of Psychology, 8(3), 475-491.

Kumar, R.K. (2008). Current clinical applications of positron emission tomography. Australian Prescriber, 31, 123-128. 
Kumpula, M. J., Orcutt, H. K., Bardeen, J. R., \& Varkovitzky, R. L. (2006). The independent predictive value of peritraumatic dissociation and experiential avoidance as prospective predictors of posttraumatic stress symptoms. Journal of Traumatic Stress, 19(4), 493-506.

La Flair, L.N., Bradshaw, C.P., Mendelson, T., \& Campbell, J. (2015). Intimate partner violence and risk of psychiatric symptoms: The moderating role of attachment. Journal of Family Violence, 30, 567-577.

Lanconi, S., Vigouroux, M., Lafuente, C., \& Chabrol, H. (2017). Problematic internet use, psychopathology, personality, defense, and coping. Computers in Human Behavior, 73, 47-54.

Lanius, R.A., Bluhm, R.L., \& Frewen, P.A. (2011). How understanding the neurobiology of complex post-traumatic stress disorder can inform clinical practice: A social cognitive and affective neuroscience approach. Acta Psychiatrica Scandinavica, $124,331-348$.

Lazarus, R.S. (1966). Psychological stress and the coping process. New York: McGraw-Hill.

Lazarus, R.S., \& Folkman, S. (1984). Stress, appraisal, and coping. New York: Springer.

LeBlanc, V. R., Regehr, C., Jelley R. B., \& Barath, I. (2008). The relationship between coping styles, performance, and responses to stressful scenarios in police recruits. International Journal of Stress Management, 15(1), 76-93. 
Le Dorze, C., \& Gisquet-Verrier, P. (2016). Sensitivity to trauma-associated cues is restricted to vulnerable traumatized rats and reinstated after extinction by yohimbine. Behavioural Brain Research, 313, 120-134.

Lee, J., Daffern, M., Ogloff, J. R., \& Martin, T. (2015). Towards a model for understanding the development of post-traumatic stress and general distress in mental health nurses. International Journal of Mental Health Nursing, 24(1), 4958.

Lee, J. S., Lee, S. W., Choi, K. S., Chung, U. S., Jeong, B. (2015). Differential mediating effects of PTSD symptom clusters on alcohol use and sleep in university students with trauma experiences: A multi-group analysis. Personality and Individual Differences, 85, 1-6.

Lee, J., Possemato, K., \& Ouimette, P.C. (2017). Longitudinal changes in combat-related posttraumatic stress disorder among Operation Enduring Freedom/Operation Iraqi Freedom/Operation New Dawn veterans with hazardous alcohol use: The role of avoidance coping. Journal of Nervous \& Mental Disease, 205(10), 805-808.

Lincoln, YS. \& Guba, EG. (1985). Naturalistic Inquiry. Newbury Park, CA: Sage Publications.

Linley, P.A., \& Joseph, S. (2004). Positive change following trauma and adversity: A review. Journal of Traumatic Stress, 17, 11-21.

Linley, P.A., Felus, A., Gillett, R., \& Joseph, S. (2011). Emotional expression and growth following adversity: Emotional expression mediates subjective distress and is moderated by emotional intelligence. Journal of Loss and Trauma, 16(5), $387-401$. 
Long, M. E., Meyer, D. L., \& Jacobs, G. A. (2007). Psychological distress among American Red Cross disaster workers responding to the terrorist attacks of September 11, 2001. Psychiatry Research, 149, 303-308.

Maack, D. J., Tull, M. T., \& Gratz K. L. (2012). Fear and avoidance of internal experiences among patients with substance use disorders and PTSD: The centrality of anxiety sensitivity. Cognitive Therapy and Research. doi 10.1007/s10608-011-9362-2.

Mairean, C. (2016). Secondary traumatic stress and posttraumatic growth: Social support as a moderator. The Social Science Journal, 53, 14-21.

Malach-Pines, A. (2004). Adult attachment styles and their relationship to burnout: A preliminary, cross-cultural investigation. Work \& Stress, 18(1), 66-80.

Malta, L.S. (2012). Allostasis: The emperor of all (trauma-related) maladies. Clinical Psychology: Science \& Practice, 19(3), 241-258.

Marchand, A., Boyer, R., Nadeau, C., Beaulieu-Prévost, D., \& Martin, M. (2015). Predictors of posttraumatic stress disorder among police officers: A prospective study. Psychological Trauma: Theory, Research, Practice, and Policy, 7(3), 212221.

Marin, M-F., Camprodon, J.A., Dougherty, D.D., \& Milad, M.R. (2014). Device-based brain stimulation to augment fear extinction: Implications for PTSD treatment and beyond. Depression and Anxiety, 31, 269-278.

Matthews, G., Zeidner, M., \& Roberts, R.D. (2002). Emotional intelligence: Science and myth. Cambridge, MA: MIT Press. 
Matusko, D.B., Kemp, R.I., Paterson, H.M., \& Bryant, R.A. (2009). The assessment of post-traumatic stress disorder for workers' compensation in emergency service personnel. Australian Psychologist, 48, 420-427.

Mayer, J.D., \& Salovey, P. (1997). What is emotional intelligence? In P. Salovey \& D.J. Sluyter (Eds.), Emotional development and emotional intelligence: Educational implications. In R.J. Sternberg (Ed.), Handbook of intelligence (pp. 3-34). Cambridge, UK: Cambridge University Press.

McCaslin, S. E., Jacobs, G. A., Meyer, D. L., Johnson-Jiminez, E., Metzler, T. J., \& Marmar, C. R. (2005). How does negative life change following disaster response impact distress among Red Cross responders? Professional Psychology: Research and Practice, 36, 246-253.

McGowan, P.O., Sasaki, A., D'Alessio, A.C., Dymov, S., Labonté, B., Szyf, Mm, Turecki, G., \& Meaney, M.J. (2009). Epigenetic regulation of the glucocorticoid receptor in human brain associates with childhood abuse. Nature Neuroscience, $12(3), 342-.348$.

McMillan, J.H., \& Schumacher, S. (2010). Research in education: Evidence-based inquiry (7th ed.). Essex, England: Pearson Education Limited.

Meyer, E.C., Zimering, R., Daly, E., Knight, J., Kamholz, B.W., \& Gulliver, S.B. (2012). Predictors of posttraumatic stress disorder and other psychological symptoms in trauma-exposed firefighters. Psychological Services, 9(1), 1-15. 
Mikulincer, M., Solomon, Z., Shaver, P.R., and Ein-Dor, T. (2014). Attachment-Related consequences of war captivity and trajectories of posttraumatic stress disorder: A 17-year long longitudinal study. Journal of Social and Clinical Psychology, 33(3), 207-228.

Mišić, B., Dunkley, B.T., Sedge, P.A., Da Costa, L., Fatima, Z., Berman, M.G., Doesburg, S.M., McIntosh, A.R., Grodecki, R., Jetly, R., Pang, E.W., \& Taylor, M.J. (2016). Post-traumatic stress constrains the dynamic repertoire of neural activity. The Journal of Neuroscience, 36(2), 419-431.

Monson, C.M., Taft, C.T., \& Fredman, S.J. (2009). Military-related PTSD and intimate relationships: From description to theory-driven research and intervention development. Clinical Psychology Review, 29, 707-714.

Morrison, L.K. (2014). Coping Techniques, World Assumptions, and Level of PostTraumatic Stress in Emergency Medical Technicians. (Publication No. 3624145) [Doctoral Dissertation, Massachusetts School of Professional Psychology]. ProQuest Dissertations and Theses Global.

Mroz, J. (2015). Predictive roles of coping and resilience for the perceived stress in nurses. Progress in Health Sciences, 5(2), 77-83.

Muijs, D. (2010). Doing Quantitative Research in Education with SPSS (2nd ed.). London: SAGE Publications.

Muller, R.T., Sicoli, L.A., \& Lemieux, K.E. (2000). Relationship between attachment styles and posttraumatic stress symptomatology among adults who report the experience of childhood abuse. Journal of Traumatic Stress, 13(2), 321-332. 
Negri, K.A. (2018). Hardiness, adult attachment style, and burnout in nurses. [Doctoral Dissertation, Walden University]. Walden Dissertations and Doctoral Studies. 5028. https://scholarworks.waldenu.edu/dissertations/5028.

Newberg, A., Alavi, A., \& Reivich, M. (2002). Determination of regional cerebral function with FDG-PET imaging in neuropsychiatric disorders. Seminars in Nuclear Medicine, 32(1), 13-34.

Nichols, M.P., \& Schwartz, R.C. (2004). Family therapy: Concepts and methods (6th ed.). Boston, MA: Pearson: Allyn and Bacon.

Nietzel, M.T., Bernstein, D.A., Kramer, G.P., \& Milich, R. (2003). Introduction to Clinical Psychology (6th ed.), Upper Saddle River: Prentice Hall.

Novara, C., Garro, M., \& Dr Rienzo, G. (2015). Coping styles and social support in emergency workers: Family as a resource. Revista Romaneasca Pentru Educatie Multidimensionala, 7(1), 129-140.

Nye, E.C., Katzman, J., Bell, J.B., Kilpatrick, J., Brainard, M., \& Haaland, K.Y. (2008). Attachment organization in Vietnam combat veterans with posttraumatic stress disorder. Attachment \& Human Development, 10(1), 41-57.

http://oak.ucc.nau.edu/rh232/courses/EPS625/Handouts/Interpreting\%20the\%20Oneway\%20MANOVA.pdf. Retrieved 10/27/2020.

O’Cleirigh, C., Ironson, G., Fletcher, M.A., \& Schneiderman, N. (2008). Written emotional disclosure and processing of trauma are associated with protected health status and immunity in people living with HIV/AIDS. British Journal of Health Psychology, 13, 81-84. 
O’Connor, M., \& Elklit, A. (2008). Attachment styles, traumatic events, and PTSD: A cross-sectional investigation of adult attachment and trauma. Attachment \& Human Development, 10(1), 59-71.

Oginska-Bulik, N., \& Kobylarczyk, M. (2014). Relation between resiliency and posttraumatic growth in a group of paramedics: The mediating role of coping strategies. International Journal of Occupational Medicine and Environmental Health, 28(4), 707-719.

Orozco Markert-Green, B. (2009). The unwed widow: Unmarried partnership loss and its implications for treatment. (Doctoral dissertation). Retrieved from https://encore.llu.edu/iii/encore/record/C__Rb1267654_SMarkertGreen__Orightresult_X 4 ?lang=eng\&suite=cobalt.

Oxford English Dictionary (2013). Oxford, UK: Oxford University Press.

Ozer, E.J., Best, S.R., Lipsey, T.L., \& Weiss, D.S. (2003). Predictors of posttraumatic stress disorder and symptoms in adults: a meta-analysis. Psychological Bulletin, 129(1), 52-73.

Parkes, K.R. (1984). Locus of control, cognitive appraisal, and coping in stressful episodes. Journal of Personality and Social Psychology, 46, 655-668.

Petrides, K.V., \& Furnham, A. (2000). On the dimensional structure of emotional intelligence. Personality and Individual Differences, 29, 313-320.

Plaxton-Hennings, C. (2004). Law enforcement organizational behavior and the occurrence of post-traumatic stress symptomology in law enforcement personnel following a critical incident. Journal of Police and Criminal Psychology, 19(2), 53-63. 
Prati, G., \& Pietrantoni, L. (2010). An application of the social support deterioration deterrence model to rescue workers. Journal of Community Psychology, 38(7), 901-917.

Prati, G., Pietrantoni, L., \& Cicognani, E. (2011). Coping strategies and collective efficacy as mediators between stress appraisal and quality of life among rescue workers. International Journal of Stress Management, 18(2), 182-195.

Reddan, A. (2015). Attachment Style: A predictor of romantic relationship tendencies and interpersonal trust in male and female emerging adults and adults. (PDF: https://pdfs.semanticscholar.org/52bc/5e3d0407254d620694a949f9344804e1b482 .pdf) [BA Honours Paper, Department of Psychology, Dublin Business School].

Regehr, C., Goldberg, G., \& Hughes, J. (2002). Exposure to human tragedy, empathy, and trauma in ambulance paramedics. American Journal of Orthopsychiatry,72, 505-513.

Richardson, G.E. (2002). The metatheory of resilience and resiliency. Journal of Clinical Psychology 58(3), 307-321.

Rinker, J. (2015). The relationship between emotional intelligence and PTSD symptoms in firefighters and emergency medical personnel. (Doctoral dissertation). Retrieved from ProQuest Dissertations and Theses database. (UMI No. 3664296). Roberts, S.T., et al (20017). Resilience as a protective factor against the development of PTSD symptoms following combat exposure in OIF/OEF veterans. Poster presentation, Veterans Affairs HSR\&D National Meeting. http://www.hsrd.research.va.gov/meetings/2007/display_abstract.cfm?RecordID= 361. 
Rzeszutek, M., Partyka, M., \& Golab, A. (2015). Temperament traits, social support, and secondary traumatic stress disorder. Professional Psychology: Research and Practice, 46(4), 213-220.

Saklofske, D.H., Austin, E.J., \& Minski, P.S. (2003). Factor structure and validity of a trait emotional intelligence measure. Personality and Individual Differences, 34, 1091-1100.

Salovey, P., \& Mayer, J. D. (1990). Emotional intelligence. Imagination, Cognition and Personality, 9, 185-211.

Salovey, P., Mayer, J. D., Goldman, S. L., Turvey, C., \& Palfai, T. P. (1995). Emotional attention, clarity, and repair: Exploring emotional intelligence using the Trait Meta-Mood Scale. In J. W. Pennebaker (Ed.), Emotion, disclosure, and health (pp. 125-154). Washington, D.C.: American Psychological Association.

Scheier, M.F., Weintraub, J.K., \& Carver, C.S. (1986). Coping with stress: Divergent strategies of optimists and pessimists. Journal of Personality and Social Psychology, 51, 1257-1264.

Schmidt, U., Kaltwasser, S.F., \& Wotjak, C.T. (2013). Biomarkers of posttraumatic stress disorder: Overview and implications for future research. Disease Markers, $35(1), 43-54$.

Schneider, K.R., Elhai, J.D., \& Gray, M.J. (2007). Coping style use predicts posttraumatic stress and complicated grief symptom severity among college students reporting a traumatic loss. Journal of Counseling Psychology, 54(3), 344-350.

Schore, A.N. (2003). Affect regulation and repair of the self. New York: Norton. 
Schutte, N.S., Malouff, J.M., \& Bhullar, N. (2009). The Assessing Emotions Scale. C. Stough, D.H. Saklofske \& J.D.A. Parker (Eds.), The Springer series on human exceptionality. Assessing emotional intelligence: Theory, research, and applications. New York: Springer Publishing, 119-135.

Schuette, N.S., Malouff, J.M., Hall, L.E., Haggerty, D.J., Cooper, J.T., Golden, C.J., \& Dornheim, L. (1998). Development and validation of a measure of emotional intelligence. Personality and Individual Differences, 25, 167-177.

Shoji, K., Jock, J., Cieslak, R., Zukowska, K., Luszczynska, A., \& Benight, C. (2014). Cultivating secondary traumatic growth among healthcare workers: The role of social support and self-efficacy. Journal of Clinical Psychology, 70(9), 831-846.

Silver, R., \& LeSauter, J. (2008). Circadian and homeostatic factors in arousal. Annals of the New York Academy of Sciences, 1129, 263-274.

Simons, J. S., Gaher, R. M., Jacobs, G. A., Meyer, D., \& Johnson-Jimenez, E. (2005). Associations between alcohol use and PTSD symptoms among American Red Cross disaster relief workers responding to the 9/11/2001 attacks. The American Journal of Drug and Alcohol Abuse, 31, 285-304.

Simpson, T., Jakupcak, M., \& Luterek, J. A. (2006). Fear and avoidance of internal experiences among patients with substance use disorders and PTSD: The centrality of anxiety sensitivity. Journal of Traumatic Stress, 19(4), 481-491.

Sinclair, V.G., \& Wallston, K.A. (2004). The development and psychometric evaluation of the Brief Resilient Coping Scale. Assessment, 11(1), 94-101. 
Singewald, N., Schmuckermair, C.S., Whittle, N., Holmes, A., \& Ressler, K.J. (2014). Pharmacology of cognitive enhancers for exposure-based therapy of fear, anxiety, and trauma-related disorders. Pharmacology \& Therapeutics, 149, 150-190.

Slanbekova, G., Chung, M.C., Abildina, S., Sabirova, R., Kapbasova, G., \& Karipbaev, B. (2017). The impact of coping and emotional intelligence on the relationship between posttraumatic stress disorder from past trauma, adjustment difficulty, and psychological distress following divorce. Journal of Mental Health, 26(4), 334341.

Smith, B. D. (2007). Sifting through trauma: Compassion fatigue and HIV/AIDS. Clinical Social Work, 35, 193-198.

Smith, J. (1989). The Nature of Social and Educational Inquiry: Empiricism Versus Interpretation. Norwood, NJ: Ablex.

Smith, S.G., Turner, B., Pati, J., Petrides, K.V., Sevdalis, N., \& Green, J.S.A. (2012). Psychological impairment in patients urgently referred for prostate and bladder cancer investigations: The role of trait emotional intelligence and perceived social support. Support Care Cancer, 20, 99-704.

Southwick, S.M., Morgan, C.A., Vythilingham, M., Krystal, J.H., \& Charney, D.S. (2004). Emerging neurobiological factors in stress resilience. PTSD Research Quarterly, 14(4), 1-6.

Stallard, P., \& Smith, E. (2007). Appraisals and cognitive coping styles associated with chronic post-traumatic symptoms in child road traffic accident survivors. Journal of Child Psychology and Psychiatry, 48(2), 194-201. 
Stanton, A.L., Kirk, S.B., Cameron, C.L., \& Danoff-Burg, S. (2000). Coping through emotional approach: Scale construction and validation. Journal of Personality and Social Psychology, 78, 1150-1169.

Stellman, J. M., Smith, R. P., Katz, C. L., Sharma, V., Charney, D. S., Herbert, R., . . . Southwick, S. (2008). Enduring mental health morbidity and social function impairment in World Trade Center rescue, recovery, and clean-up workers: The psychological dimensions of an environmental health disaster. Environmental Health Perspectives, 116, 1248-1253.

Stokes, L.D., \& Jackson, Y. (2014). Community violence and psychological adjustment in youth: Role of emotional-social intelligence. Journal of Child Adolescent Trauma, 7, 17-26.

Stone, A.A., Kennedy-Moore, E., Newman, M.G., Greenberg, M., \& Neale, J.M. (1992). Conceptual and methodological issues in current coping assessments. In B. N. Carpenter (Ed.) Personal Coping: Theory, Research, and Application (pp. 15-29).

Stone, D.R. (1998). Coping styles, personality traits, and social factors among paramedics with and without PTSD symptomatology.

Suddaby, R. (2006). From the editors: What grounded theory is not. Academy of Management Journal, 49(4), 633-642.

Sveen, J., Bondjers, K., \& Willebrand, M. (2016). Psychometric properties of the PTSD Checklist for DSM-5: A pilot study. European Journal of Psychotraumatology, 7, 1-7. 
Thomaes, K., Engelhard, I.M., Sijbrandij, M., Cath, D.C., \& Van den Heuvel, O.A. (2016). Degrading traumatic memories with eye movements: A pilot functional MRI study in PTSD. European Journal of Psychotraumatology, 7, 1-10.

Tolin, D.F., \& Foa, E.B. (2006). Sex differences in trauma and posttraumatic stress disorder: A quantitative review of 25 years of research. Psychological Bulletin, 132(6), 959-992.

Treadway, M.T., Grant, M.M., Ding, Z., Hollon, S.D., Gore, J.C., \& Shelton, R.C. (2009). Early adverse events, HPA activity, and rostral anterior cingulate volume in MDD. Public Library of Science One, 4(3), e4887.

Trochim, W.M.K. (March 2020). Qualitative validity. https://conjointly.com/kb/qualitative-validity/

Turunen, T., Haravouri, H., Punamaki, R-L., Suomalainen, L., \& Marttunen, M. (2014). The role of attachment in recovery after a school-shooting trauma. European Journal of Psychotraumatology, 5(22728), 1-10.

Tyrka, A.R., Burgers, B.A., Philip, N.S., Price, L.H., \& Carpenter, L.L. (2013). The neurobiological correlates of childhood adversity and implications for treatment. Acta Psychiatry Scandinavica, 128(6), 434-447.

Van Boven, R.W., Harrington, G.S., Hackney, D.B., Ebel, A., Gauger, G., Bremner, J.D., D'Esposito, M., Detre, J.A., Haacke, E.M., Jack, C.R. Jr., Jagust, W.J., Le Bihan, D., Mathis, C.A., Mueller, S., Mukherjee, P., Schuff, N., Chen, A., \& Weiner, M.W. (2009). Advances in neuroimaging of traumatic brain injury and posttraumatic stress disorder. Journal of Rehabilitation Research Development, $46,717-757$. 
Van Deusen, K. M. \& Way, I. (2006). Vicarious trauma: An exploratory study of the impact of providing sexual abuse treatment on clinicians' trust and intimacy. Journal of Child Sexual Abuse, 15, 69-85.

Van Manen, M. (1990a). Hermeneutic phenomenological reflection. In M. Van Manen (Ed.), Researching lived experience (pp. 77-109). Albany: State University of New York Press.

Van Manen, M. (1990b). Researching Lived Experience: Human Science for an Action Sensitive Pedagogy. Albany, NY: State University of New York Press.

Vashdi, D. R., Bamberger, P. A., Bacharach, S. (2012). Effects of job control and situational severity on the timing of help-seeking. Journal of Occupational Health Psychology, 17(2), 206-19.

Veer, I.M., Oei, N.Y.L., Spinhoven, P., Van Buchem, M.A. Elizinga, B.M., \& Rombouts, S.A.R.B., (2012). Endogenous cortisol is associated with functional connectivity between the amygdala and medial prefrontal cortex. Psychoneuroendocrinology, 37(7), 1039-1047.

Vera, H.A. (2013). Resilience theory and trauma theory applied to adult women incest survivors. Theses, Dissertations, and Projects. Paper 601.

Vogel, J.M., Cohen, A.J., Habib, M.S., \& Massey, B.D. (2004). In the wake of terrorism: Collaboration between a psychiatric department and a center for emergency medical services (EMS) to support EMS workers and their families. Families, Systems, and Health, 22(1), 35-46. 
Wagner, S.L., \& Martin, C.A. (2102). Can firefighters' mental health be predicted by emotional intelligence and proactive coping? Journal of Loss and Trauma, 17, 56-72.

Wakita, K., Imahori, Y., Ido, T., Fujii, H., Horii, H., Shimizu, M., Nakajima, K., Nakamura, T., \& Kanatsuna, T. (2000). Simplification for measuring input function of FDG PET: investigation of 1-point blood sampling method. Journal of Nuclear Medicine, 41(9), 1484-1490.

Watson, N.V., \& Breedlove, S.M. (2016). The Mind's Machine: Foundations of Brain and Behavior (2nd Ed.). Sunderland, MA: Sinauer Associates, Inc.

Weathers, F.W., Litz, B.T., Keane, T.M., Palmieri, P.A., Marx, B.P., \& Schnurr, P.P. (2013). The PTSD Checklist for DSM-5 (PCL-5) - Standard [Measurement instrument]. Scale available from the National Center for PTSD at www.ptsd.va.gov.

Willing, J. (2011). Firefighter suicide prevention: The company officer's role. Retrieved 12/6/2017 from http://www.firerescue1.com/cod-company-officerdevelopment/articles/1080052-Firefighter-suicide-prevention-The-companyofficers-role/.

Wilson, J.P. (1995). Traumatic events and PTSD prevention. In B. Raphael \& E.D. Barrows (Eds.), The handbook of preventative psychiatry (pp. 281-296). Amsterdam, the Netherlands: Elsevier North-Holland.

Woodhouse, S., Ayers, S., \& Field, A.P. (2015). The relationship between adult attachment style and posttraumatic stress symptoms: A meta-analysis. Journal of Anxiety Disorders, 35, 103-117. 
Yehuda, R., \& Flory, J.D. (2007). Differentiating biological correlates of risk, PTSD, and resilience following trauma exposure. Journal of Traumatic Stress, 20(4), $435-447$.

Yu, X., \& Zhang, J. (2007). Factor analysis and psychometric evaluation of the ConnorDavidson Resilience Scale (CD-RISC) with Chinese people. Social Behavioral Personality, 35, 19-30.

Yusoff, N., Low, W.Y., \& Yip, C.H. (2010). Reliability and validity of the Brief COPE Scale (English version) among women with breast cancer undergoing treatment of adjuvant chemotherapy: A Malaysian study. Medical Journal of Malaysia, 65(1), $41-44$.

Zaki, J. (2014). Empathy: A motivated account. Psychological Bulletin, 140(6), 16081647.

Zakin, G., Solomon, Z., \& Neria, Y. (2003). Hardiness, attachment style, and long term psychological distress among Israeli POWs and combat veterans. Personality and Individual Differences, 34(5), 819-829.

Zeidner, M., \& Endler, N.S. (1996). Handbook of coping. New York, NY: James Wiley. Zucker, T.L., Samuelson, K.W., Muench, F., Greenberg, M.A. \& Gevirtz, R.N. (2009). The Effects of Respiratory Sinus Arrhythmia Biofeedback on Heart Rate Variability and Posttraumatic Stress Disorder Symptoms: A Pilot Study. Applied Psychophysiology Biofeedback, 34, 135-143.

Zuckerman, M. (1999). Vulnerability to psychopathology. Washington, D.C.: American Psychological Association. 
APPENDICES 


\section{APPENDIX A}

Advertisement Script 


\section{Advertisement Script}

You are invited to participate in a doctoral student research study about the field experiences of Emergency Medical Technicians (EMT-B's, EMT-I's, EMT-P's) who have in the past or who currently work in the private sector and who have been exposed to traumatic events. Your participation will require approximately 30 minutes. The goal of this research is to understand your experiences from your perspective, and to identify factors that both help and hinder the presence of PTSD. We hope that the information collected from this study will provide a better understanding of how PTSD becomes present in the lives of EMTs working in the private sector, and whether there are differences in those who wind up with PTSD and those who don't. Participants must be 18-years-of-age or older and must have worked in the private sector either presently or in the past. Participants who currently work in the public sector (fire department, police department, etc.) are ineligible for the study. Research participants will be asked to fill out a background questionnaire which will include minimal demographic information, and to answer a series of short questionnaires related to their field experiences.

Confidentiality will be maintained on all research participants. No compensation will be provided to the participants of the study, nor will there be any financial costs incurred by the participants of the study. If you are an EMT who has worked in the private sector and would like to participate in this study, please contact either Brenda Markert-Green, doctoral student at Andrews University Graduate Psychology and Counseling Department, by emailing markertb@ andrews.edu, or by calling (269) 471-3473, or Dennis Waite, Ed.D. Primary Supervising Investigator, at (269) 208-2532. 


\section{APPENDIX B}

Informed Consent 


\title{
Informed Consent for Participation in Research for Participants
}

\author{
ANDREWS UNIVERSITY \\ School of Education \\ Department of Graduate Psychology and Counseling \\ Berrien Springs, Michigan \\ (269) 471-3473
}

\section{INFORMED CONSENT}

\author{
Coping Skills, Emotional Intelligence, Attachment, and Resiliency \\ on Post-Traumatic Stress Disorder in Emergency Medical Technicians
}

\section{Purposes and Procedures}

You are invited to participate in a doctoral student research study about the field experiences of Emergency Medical Technicians (EMT-B's, EMT-I's, EMT-P's) who have in the past or who currently work in the private sector and who have been exposed to traumatic events. Your participation will require approximately 60 minutes. The goal of this research is to understand your experiences from your perspective, and to identify factors that both help and hinder the presence of PTSD. We hope that the information collected from this study will provide a better understanding of how PTSD becomes present in the lives of EMTs working in the private sector, and whether there are differences in those who wind up with PTSD and those who don't.

You will be provided with a URL which will take you to an online survey platform where you will answer the questions needed for the study. You will be asked to read and understand this consent form and to answer some demographic questions. You will also be asked to answer several pre-screening questions. Following this, you will be provided with and asked to complete several surveys and questionnaires related to your experience. There will be a series of 5 short questionnaires to answer.

We wish to thank you for assisting us by your willingness to participate in this study. Your help with this research project will provide needed information that may help private sector EMTs, and possibly others who have similar experiences. If you would like to ask more questions about this project, you can contact the principle investigator through the provided contact information. Requests for information does not mandate participation.

\section{Risks}

Sharing your experiences about traumatic events and about PTSD is a sensitive matter; therefore, we want you to know that all of your personal information will be kept confidential. Even though we will use your name on the surveys and questionnaires you will be completing, when we examine the questionnaires and surveys, we will use only a 
number to identify you. All of your information and your responses will be kept strictly confidential, and digital data will be stored in secure computer files after it is entered.

Any report of this research that is made available to the public will not include your name or any other individual information by which you could be identified.

We would like to ask that you answer all the questions honestly. Some questions are personal and may make you feel uncomfortable. You are free to withdraw from the study or to decide that you don't want to finish at any time in the process, even if it's right in the middle of filling out a survey or questionnaire. Everything we ask you is so that we can understand your experience as much as we can, and to be able to use this information to help other people like you who work in the private sector of the field.

You may recognize at some point during this interview that you are having a difficult time coping with your experiences. If so, you may want to pursue additional counseling. Since this study may include participants from geographically separated metropolitan areas, it is difficult for us to provide referrals for each city. Most employee programs provide for either an employee assistance program or with medical insurance that would provide coverage for you to see a mental health professional. We understand, however, that you may wish to avoid going through official channels. In that case, another resource that you may wish to use is the Psychology Today find a therapist website. The URL for this is as follows https://therapists.psychologytoday.com/rms/. By using your city/state information, or just the zip code, you can locate a licensed therapist who may assist you at your own expense.

\section{Benefits}

Although this research may not benefit you directly, it will help us to learn from your experience so that we can help other people who may be experiencing distress from their experiences in the field. This research may provide a greater knowledge about how EMTs (EMT-B's, EMT-I's, and EMT-P's) who work in the private sector deal with the traumatic events that may be inherent in your work as a private sector EMT. With such knowledge, clinicians may be able to design better treatments to help these individuals.

\section{Confidentiality}

As stated above, your name will not be used in any way that could identify you. All of your personal information will be kept confidential. Even though we will use your name on the surveys and questionnaires you will be completing, when we examine the questionnaires and surveys, we will use only a number to identify you. All of your information and your responses will be kept strictly confidential, and digital data will be stored in secure computer files after it is entered. Any report of this research that is made available to the public will not include your name or any other individual information by which you could be identified. 


\section{Participants Rights}

Please remember that your participation in this study is completely voluntary. If you decide that you do not want to participate, you may stop at any time.

\section{Additional Costs}

There is no cost to you for participating in this study.

\section{Reimbursement}

There is no reimbursement to you for participating in this study.

\section{Impartial Third Party Consent}

If you want to talk to someone who is not involved in this study about any complaints that you have, you may call the Andrews University Institutional Research Board by calling (269) 471-3042. You can also send them an e-mail if you would like at research@andrews.edu.

\section{Informed Consent Statement}

Completing this survey indicates that you are 18 years of age or older and indicates your consent to participate in the research. You may print out this screen for informed consent if you would like a copy of it, or, you may email the principle researcher, Brenda Markert-Green, at markertb@andrews.edu, to receive a copy. If you have any questions about your rights or treatment as a research participant, you may contact the Andrews University Institutional Research Board by calling (269) 471-3042 or by emailing at research@andrews.edu. By signing your name, you are not giving up any of your rights, and it does not keep the researcher, Andrews University, or anyone else involved in this study from their responsibility to you as our participant. You may call the student researcher, Brenda Markert-Green, D.M.F.T., at (269) 471-3473 or her research supervisor, Dennis Waite, Ed.D., at (269) 208-2532 if you have anything else you want to ask, or if you have any other concerns.

We thank you so much for your participation!

Research Advisor: Dennis Waite, Ed.D.

Assistant Professor of Psychology

Andrews University

Principle Investigator: Brenda Markert-Green, Psychology Graduate Student Andrews University, School of Education 


\section{APPENDIX C}

Demographic Questions 


\section{Demographic Questions}

1. Please indicate your age.

2. Please indicate your gender identity.

3. Please indicate your current level as an EMT.

4. Please indicate your cultural background.

5. Please indicate the amount of time you have worked in the field at your current level; if less than a year, indicate the number of months.

6. Please indicate the amount of time you have worked in the field at your previous level; if less than a year, indicate the number of months, and indicate your previous level as an EMT.

7. Please indicate the total amount of time you have worked in the field as an EMT; if less than a year, indicate the number of months.

8. Are you currently working as an EMT in the public sector of the field (fire department, police department, hospital)?

9. Have you in the past, or are you now working as an EMT in the private sector of the field, or was your last job as an EMT in the private sector of the field (private ambulance company)? 


\section{APPENDIX D}

Survey Instruments 


\section{PCL-5}

Instructions: Below is a list of problems that people sometimes have in response to a very stressful experience. Please read each problem carefully and then circle one of the numbers to the right to indicate how much you have been bothered by that problem in the past month.

\begin{tabular}{|c|c|c|c|c|c|}
\hline In the past month, how much were you bothered by: & $\begin{array}{c}\text { Not at } \\
\text { all }\end{array}$ & $\begin{array}{c}\text { A little } \\
\text { bit }\end{array}$ & Moderately & $\begin{array}{l}\text { Quite } \\
\text { a bit }\end{array}$ & Extremely \\
\hline $\begin{array}{l}\text { 1. Repeated, disturbing, and umwanted memories of the } \\
\text { stressful experience? }\end{array}$ & (a) & (1) & (2) & (3) & (4) \\
\hline 2. Repeated, disturbing dreams of the stressful experience? & (0) & (1) & (2) & (3) & (4) \\
\hline $\begin{array}{l}\text { 3. Suddenly feeling or acting as if the stressful experience were } \\
\text { actually happening again (as if you were actually back there. } \\
\text { reliving it)? }\end{array}$ & (0) & (1) & (2) & (3) & (4) \\
\hline $\begin{array}{l}\text { 4. Feeling very upset when something reminded you of the } \\
\text { stressful experience? }\end{array}$ & (0) & (1) & (2) & (3) & (4) \\
\hline $\begin{array}{l}\text { 5. Hawing strong physical reactions when something reminded } \\
\text { you of the stressful experience for example, heart } \\
\text { pounding, trouble breathing, sweating|? }\end{array}$ & (0) & (1) & (2) & (3) & (4) \\
\hline $\begin{array}{l}\text { 6. Avoiding memories, thoughts, or feelings related to the } \\
\text { stressful experience? }\end{array}$ & (0) & (1) & (2) & (3) & (4) \\
\hline $\begin{array}{l}\text { 7. Avoiding external reminders of the stressful experience (for } \\
\text { example, people, places, conversations, activities, objects, or } \\
\text { situations)? }\end{array}$ & (0) & (1) & (2) & (3) & (4) \\
\hline $\begin{array}{l}\text { 8. Trouble remembering important parts of the stressful } \\
\text { experience? }\end{array}$ & (0) & (1) & (2) & (3) & (4) \\
\hline $\begin{array}{l}\text { 9. Hawing strong negative beliefs about yourself, other people, } \\
\text { or the world (for example, having thoughts such as } 1 \text { am } \\
\text { bad, there is something seriously wrong wth me, } \\
\text { no one can be trusted, the world is completely dangerous)? }\end{array}$ & (0) & (1) & (2) & (3) & (4) \\
\hline $\begin{array}{l}\text { 10. Blaming yourself or someone else for the stressful } \\
\text { experience or what happened after it? }\end{array}$ & (0) & (1) & (2) & (3) & (4) \\
\hline $\begin{array}{l}\text { 11. Having strong negative feelings such as fear, horror, anger. } \\
\text { guilt, or shame? }\end{array}$ & (0) & (1) & (2) & (3) & (4) \\
\hline 12. Loss of interest in activities that you used to enjoy? & (0) & (1) & (2) & (3) & (4) \\
\hline 13. Feeling distant or cut off from other people? & (0) & (1) & (2) & (3) & (4) \\
\hline $\begin{array}{l}\text { 14. Trouble experiencing positive feelings (for example, being } \\
\text { unable to feel happiness or have loving feelings for people } \\
\text { close to you)? }\end{array}$ & (0) & ) & (2) & (3) & (4) \\
\hline 15, Irritable behavior, angry outbursts, or acting aggressively? & (0) & (1) & (2) & (3) & (4). \\
\hline $\begin{array}{l}\text { 16. Taking too many risks or doing things that could cause you } \\
\text { harm? }\end{array}$ & (a) & (1) & (2) & (3) & (4) \\
\hline 17. Being "superalert" or watchful or on guard? & (0) & (1) & (2) & (3) & (4) \\
\hline 18. Feeling fumpy or easily startied? & (0) & (1) & (2) & (3) & (4) \\
\hline 19. Hawing difficulty concentrating? & (6) & (1) & (2) & (3) & (4) \\
\hline 20. Trouble falling or staying asleep? & (0) & (1) & (2) & (3) & (4) \\
\hline
\end{tabular}




\section{Brief COPE}

These items deal with ways you've been coping with the stress in your life since you found out you were going to have to have this operation. There are many ways to try to deal with problems. These items ask what you've been doing to cope with this one. Obviously, different people deal with things in different ways, but I'm interested in how you've tried to deal with it. Each item says something about a particular way of coping. I want to know to what extent you've been doing what the item says. How much or how frequently. Don't answer on the basis of whether it seems to be working or not-just whether or not you're doing it. Use these response choices. Try to rate each item separately in your mind from the others. Make your answers as true FOR YOU as you can.

$1=\mathrm{I}$ haven't been doing this at all

$2=$ I've been doing this a little bit

$3=$ I've been doing this a medium amount

$4=$ I've been doing this a lot

1. I've been turning to work or other activities to take my mind off things.

2. I've been concentrating my efforts on doing something about the situation I'm in.

3. I've been saying to myself "this isn't real.".

4. I've been using alcohol or other drugs to make myself feel better.

5. I've been getting emotional support from others.

6. I've been giving up trying to deal with it.

7. I've been taking action to try to make the situation better.

8. I've been refusing to believe that it has happened.

9. I've been saying things to let my unpleasant feelings escape.

10. I've been getting help and advice from other people.

11. I've been using alcohol or other drugs to help me get through it.

12. I've been trying to see it in a different light, to make it seem more positive.

13. I've been criticizing myself.

14. I've been trying to come up with a strategy about what to do.

15. I've been getting comfort and understanding from someone.

16. I've been giving up the attempt to cope.

17. I've been looking for something good in what is happening.

18. I've been making jokes about it.

19. I've been doing something to think about it less, such as going to movies, watching TV, reading, daydreaming, sleeping, or shopping.

20. I've been accepting the reality of the fact that it has happened.

21. I've been expressing my negative feelings.

22. I've been trying to find comfort in my religion or spiritual beliefs.

23. I've been trying to get advice or help from other people about what to do.

24. I've been learning to live with it.

25. I've been thinking hard about what steps to take.

26. I've been blaming myself for things that happened.

27. I've been praying or meditating.

28. I've been making fun of the situation. 


\section{The Schutte Self Report Emotional Intelligence Test (SSEIT)}

Instructions: Indicate the extent to which each item applies to you using the following scale:

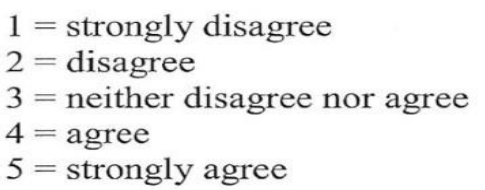

1. I know when to speak about my personal problems to others

2. When I am faced with obstacles, I remember times I faced similar obstacles and overcame them

3. I expect that I will do well on most things I try

4. Other people find it easy to confide in me

5. I find it hard to understand the non-verbal messages of other people*

6. Some of the major events of my life have led me to re-evaluate what is important and not important

7. When my mood changes, I see new possibilities

8. Emotions are one of the things that make my life worth living

9. I am aware of my emotions as I experience them

10. I expect good things to happen

11. I like to share my emotions with others

12. When I experience a positive emotion, I know how to make it last

13. I arrange events others enjoy

14. I seek out activities that make me happy

15. I am aware of the non-verbal messages I send to others

16. I present myself in a way that makes a good impression on others

17. When I am in a positive mood, solving problems is easy for me

18. By looking at their facial expressions, I recognize the emotions people are experiencing

19. I know why my emotions change

20. When I am in a positive mood, I am able to come up with new ideas

21. I have control over my emotions

22. I easily recognize my emotions as I experience them

23. I motivate myself by imagining a good outcome to tasks I take on

24. I compliment others when they have done something well

25. I am aware of the non-verbal messages other people send

26. When another person tells me about an important event in his or her life, I almost feel as though I have experienced this event myself

27. When I feel a change in emotions, I tend to come up with new ideas

28. When I am faced with a challenge, I give up because I believe I will fail*

29. I know what other people are feeling just by looking at them

30. I help other people feel better when they are down

31. I use good moods to help myself keep trying in the face of obstacles

32. I can tell how people are feeling by listening to the tone of their voice 
33. It is difficult for me to understand why people feel the way they do* 


\section{Measure of Attachment Qualities}

Respond to each of the following statements by expressing how much you agree with it (if you do generally agree) or how much you disagree with it (if you generally disagree). Make all your responses on the answer sheet only. Do not leave any items blank. Please be as accurate as you can be throughout, and try especially hard not to let your answer to any one item influence your answer to any other item. Treat each one as though it is completely unrelated to the others. There are no right or wrong answers, you are simply to express your own personal feelings and opinions. Choose from these reponse options:

$1=\mathrm{I}$ DISagree with the statement a lot

$2=\mathrm{I}$ DISagree with the statement a little

$3=\mathrm{I}$ agree with the statement a little

$4=$ I agree with the statement a lot

1. When I'm close to someone, it gives me a sense of comfort about life in general.

2. I often worry that my partner doesn't really love me.

3. I have trouble getting others to be as close as I want them to be.

4. I find it easy to be close to others.

5. I often worry my partner will not want to stay with me.

6. Others want me to be more intimate than I feel comfortable being.

7. It feels relaxing and good to be close to someone.

8. I am very comfortable being close to others.

9. I don't worry about others abandoning me.

10. My desire to merge sometimes scares people away.

11. I prefer not to be too close to others.

12. I find others are reluctant to get as close as I would like.

13. I get uncomfortable when someone wants to be very close.

14. Being close to someone gives me a source of strength for other activities.

Items 4, 8, and 9 are reverse coded.

Security $=$ Items 1,7 , and 14 .

Avoidance $=$ Items 4, 6, 8, 11, and 13 .

Ambivalence-worry $=$ Items 2, 5, and 9 .

Ambivalence-merger $=$ Items 3, 10, and 12 . 


\section{Connor-Davidson Resilience Scale 25 (CD-RISC-25) (C)}

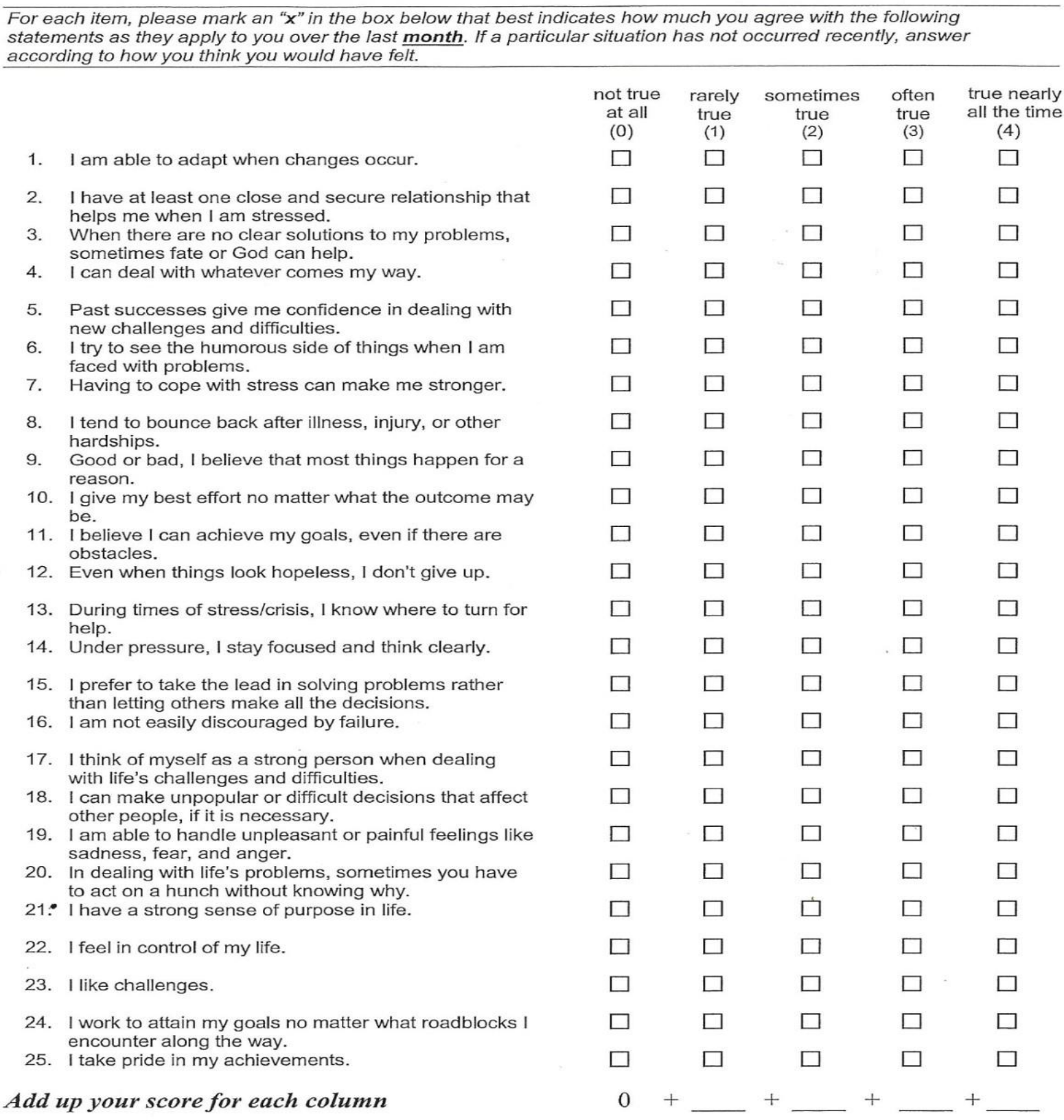

Add each of the column totals to obtain CD-RISC score

All rights reserved. No part of this document may be reproduced or transmitted in any form without permission in writing from Dr. Davidson at mail@,cd-risc.com. . Copyright (c) 2001, 2018 by Kathryn M. Connor, M.D., and Jonathan R.T. Davidson. M.D. 